\title{
AN ECONOMIC ANALYSIS OF SUGAR SWEETENED BEVERAGE TAXES - A MODEL WITH SUBSTITUTES AND \\ COMPLEMENTS
}

\author{
BY \\ NINGXIN DING
}

\begin{abstract}
A thesis
submitted to Victoria University of Wellington

in fulfilment of the requirements for the degree of

Doctor of Philosophy
\end{abstract}

Victoria University of Wellington

2021 


\begin{abstract}
Studies have shown that excess sugar intake is one of the potential causes of obesity and diabetes. As a result, taxing sugar sweetened beverages (SSBs) has been put forward as a possible solution. However, SSB taxes may not be effective, as consumers may switch to other untaxed drinks. In addition, there are gaps regarding (1) the socially optimal tax rate in New Zealand, (2) which tax base is the most favorable at its socially optimal level, and (3) whether taxing all beverages is superior to taxing only SSBs. Given these, this study addresses the socially optimal tax rate, taking into account substitutes and complements. It also explores the most efficient tax base, and whether beverage taxes are superior to SSB taxes.

The study starts from constructing a utility-maximization model of the optimal corrective tax, which allows substitution and complementary effects. Since the marginal harm from SSBs is included as a component of the optimal tax formula, a contingent valuation survey is conducted to estimate people's willingness-to-pays for health risk reductions, the results of which are further used to measure the monetary value of harm associated with internalities. Moreover, cost analyses using a Markov model and the UK Prospective Diabetes Study model are applied to estimate the harm associated with externalities. Finally, effects of taxes on social welfare are modeled, the result of which can inform the question of which tax base is the most efficient, and whether beverage taxes are superior to SSB taxes or not.

Our estimate of the optimal tax rate suggests that the prices of SSBs in New Zealand should probably increase by $100 \%$ to $200 \%$. A beverage tax by calories is the most favorable option, as it has a perfect relationship with the harmful ingredients. Whether taxing all beverages by price or by litres is superior to taxing only SSBs depends on the calories substitutes contain, and the magnitude of substitution effects. When there are strong substitution effects, and substitutes contain low energy, taxing only SSBs is better than taxing all beverages by price or by litres.
\end{abstract}




\section{Acknowledgements}

First and foremost, I would like to thank my principal supervisor Dr. Jaikishan Desai. His comments are critical and helpful. Without his guidance, I would have had a much tougher experience. I am inspired by his wisdom.

Second, I would like to express my gratitude to Dr. Paul Calcott. He always puts aside time for me, and spends lots of time on reading, checking, and revising my drafts. His feedbacks are crucial and always on time. I am benefit from his knowledge.

Furthermore, I would like to thank those people who participated in my survey. I am immensely grateful for their time and help.

Finally, I would like to express my gratitude to my family and friends for their love, encouragement, and support. 


\section{Contents}

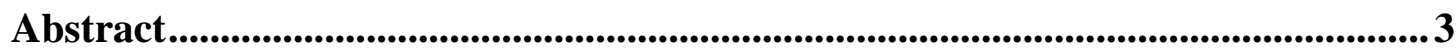

Acknowledgements ...........................................................................................................................4

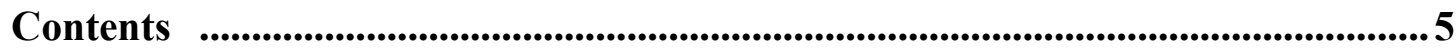

List of Tables

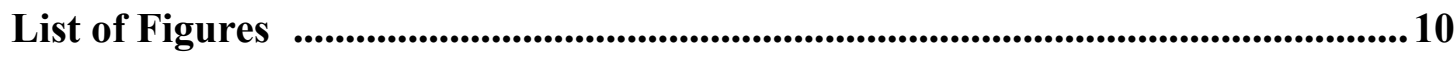

\section{Introduction}

1.1 Background ........................................................................................................................ 13

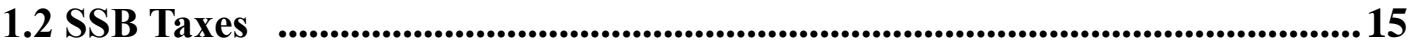

1.3 Statement of the Problem ...........................................................................................20

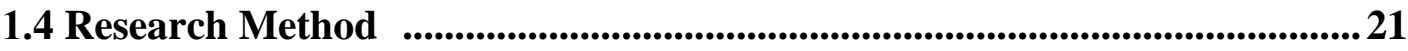

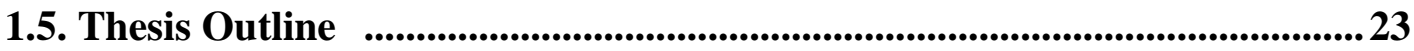

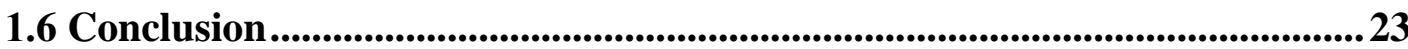

2. A Review of Literature on SSB taxes

2.1 Introduction .............................................................................................................25

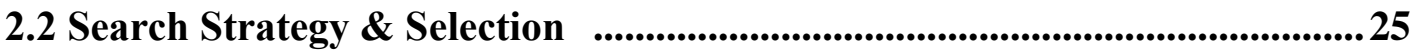

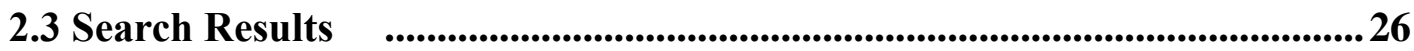

2.4 Socially-Optimal Level ..........................................................................................227

2.5. Comparison of Tax Bases ...............................................................................29

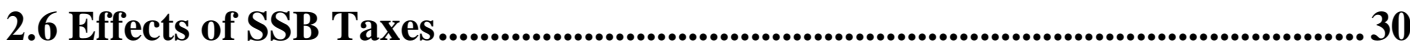

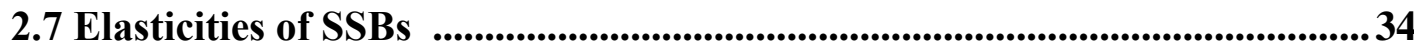

2.8 Benefits of SSB Taxes, Internalities \& Externalities of SSBs

2.8.1 Benefits of SSB Taxes

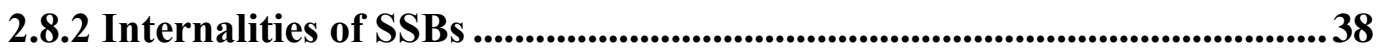




\subsection{Other Concerns}

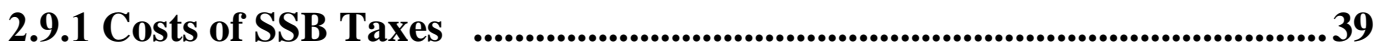

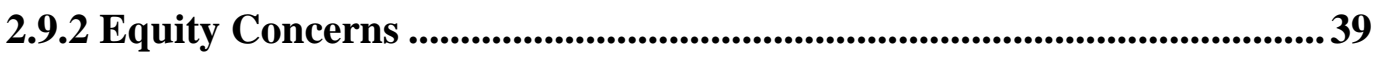

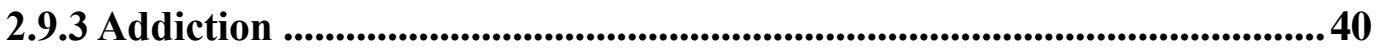

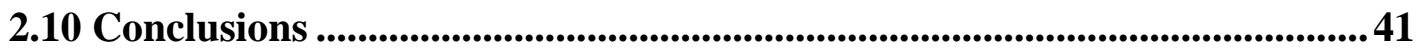

\section{Utility-Maximization Model}

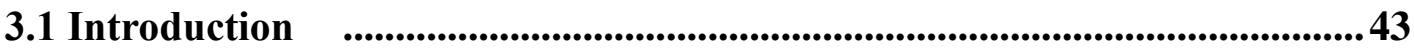

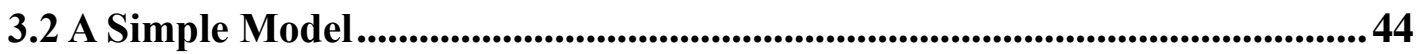

3.3 An Extended Model \& Tax Impacts

3.3.1 An Extended Model ................................................................................................46

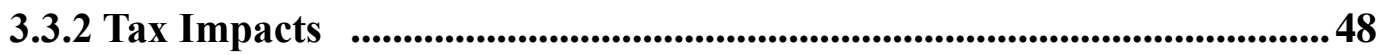

3.3.3 The Socially Optimal Levels for Sugary Drink Taxes...............................50

3.4 Constructing a Welfare Measurement .....................................................................50

3.5 Discussion \& Conclusion $\quad$.....................................................................52

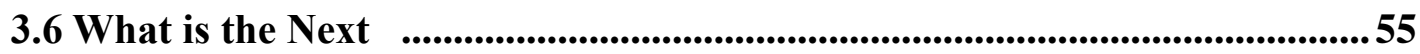

4. Measuring the Harm of SSBs associated with Internalities

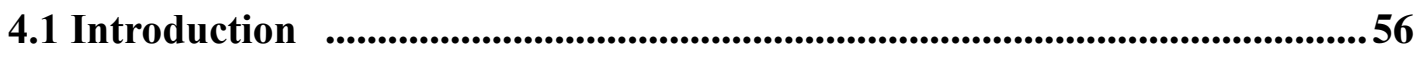

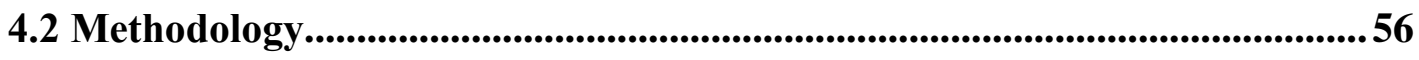

4.2.1 Increased health risks $\quad$...........................................................................5

4.2.2 SSB Consumption..................................................................................................60

4.2.3 Loss from Diseases.........................................................................61

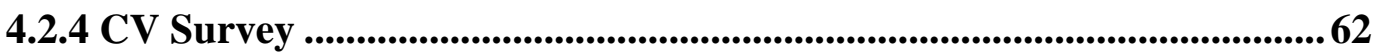

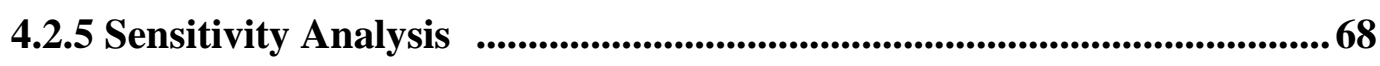

\subsection{Results}


4.3.1 The Pilot Survey Using Students ............................................................69

4.3.2 The Survey on Facebook ....................................................................................69

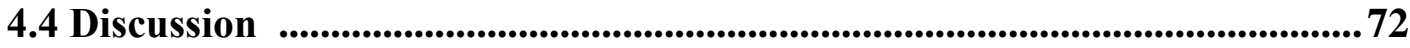

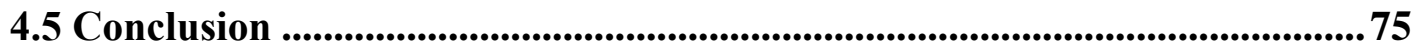

5. Measuring the Harm of SSBs associated with Externalities

5.1 Introduction \& Objective

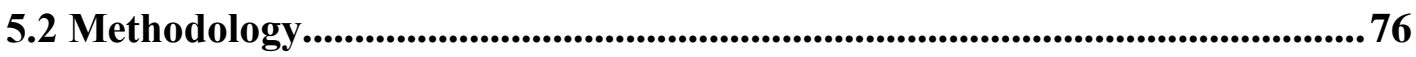

5.3 Costs

5.3.1 Source of Cost Data ....................................................................................77

5.3.2 Costing Method ......................................................................................................... 78

5.3.3 Stroke Costs.......................................................................................................79

5.3.4 IHD Costs ...................................................................................................................8 84

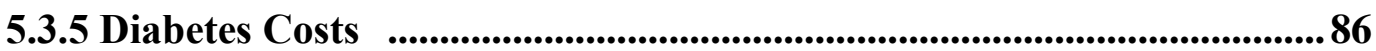

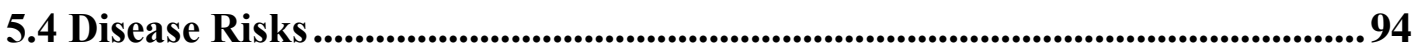

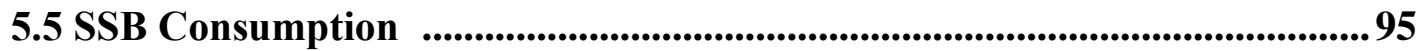

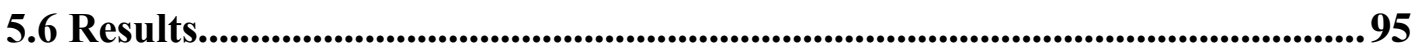

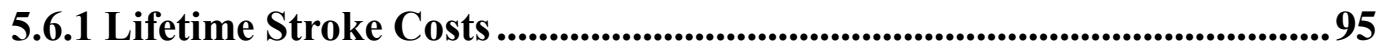

5.6.2 Lifetime IHD Costs ............................................................................................96

5.6.3 Lifetime Diabetes Costs............................................................................97

5.6.4 Total Harm from Externalities .............................................................98

5.7 Discussion ............................................................................................................98

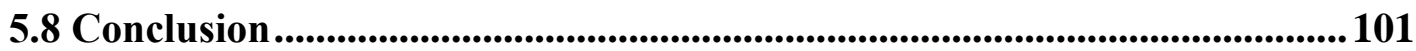

6. Simulation Using Real World Data

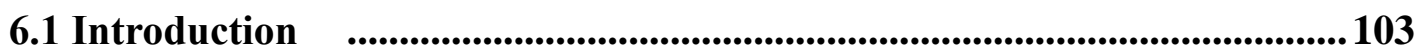


6.2 Method \& Data Source

\subsection{Results}

6.3.1 Base Case

6.3.2 Sensitivity Analysis ................................................................................................. 108

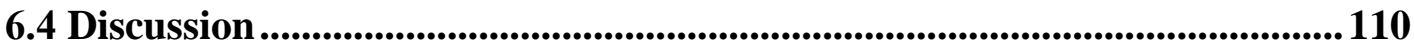

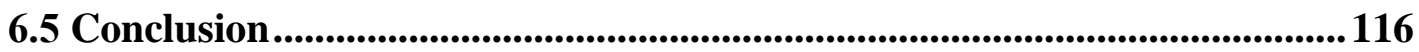

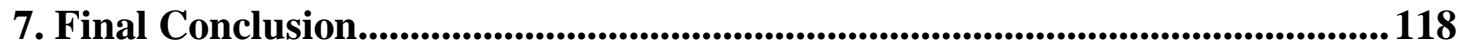

Appendix I: Literature Review ..................................................................................................128

Appendix II: Information for Chapter Four - The CV study .................................144

Appendix III: Information for IHD and Stroke Costs ................................................. 174

Appendix IV: Information for Diabetes Costs \& the UKPDS Model........................ 182

Appendix V: Information for Chapter Six .........................................................................204

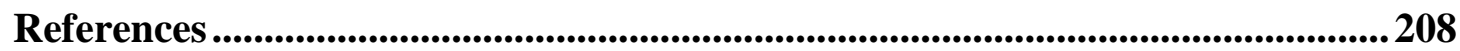




\section{List of Tables}

Table 2.1: Basic Information on Non-Experiment Studies .......................................128

Table 2.2: Main Findings of Non-Experiment Studies .............................................. 132

Table 2.3: Main Findings of Non-Experiment Studies (Continued)............................. 137

Table 2.4: Basic Information on Experimental Studies .................................................. 140

Table 2.5: Main Findings of Experimental Studies ................................................142

Table 4.1: Summary of the Evidence........................................................................................ 144

Table 4.2: Binary Logistic Regression Analysis on Explain Decisions in the Pilot

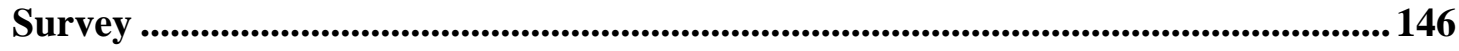

Table 4.3: Summary Statistics of Variables and Missing Data for the Survey on

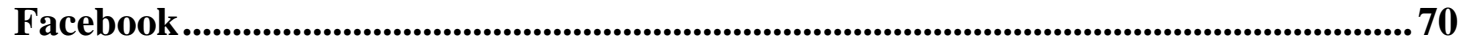

Table 4.4: Binary Logistic Regression Analysis on Explain Decisions (Facebook

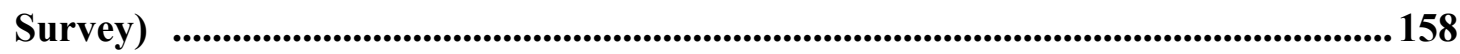

Table 4.5: Marginal Harm of SSBs per Litre ..............................................................

Table 5.1: Stroke Costs: The Most Serious Situation .........................................................176

Table 5.2 Stroke Costs: the Most Conservative Situation................................................ 177

Table 5.3 Stroke Costs: Base Case ................................................................................178

Table 5.3.1 Stroke Costs: Base Case (Reduced Table) ............................................................8 82

Table 5.4 IHD Costs: The Most Serious Situation ......................................................179

Table 5.5 IHD Costs: the Most Conservative Situation .....................................................180

Table 5.6 IHD Costs: Base Case.........................................................................................181

Table 5.7 Demographic Information.....................................................................................88

Table 5.8 Dialysis Costs in New Zealand ......................................................................182

Table 5.9 UKPDS Results: Diabetes Total Costs \& Life Expectancy (Base Case) .....97 
Table 5.10: UKPDS Results: Diabetes Costs without Complications, Costs of Complications, \& the Possible Ranges of Total Costs ................................................... 182

Table 5.11 New Zealand Diabetes Patient Population \& Incidence by Year ............ 184

Table 5.12: Formulas Used in the UKPDS model ...................................................... 184

Table 5.13 Variable Definition in the UKPDS Model................................................ 185

Table 5.14: Heart Failure Costs: the Most Serious Situation ...................................... 186

Table 5.15 Heart Failure Costs: The Most Conservative Situation .............................. 187

Table 5.16: Heart Failure Costs: Base Case ...................................................................188

Table 5.17 MI Costs: the Most Serious Situation ............................................................... 189

Table 5.18: MI Costs: the Most Conservative Situation...............................................190

Table 5.19 MI Costs: Base Case ................................................................................................... 191

Table 5.20 Costs of Blindness ..........................................................................196

Table 5.21 Costs of Amputation .............................................................................................. 183

Table 5.22: Costs of Renal Failure: The Most Serious Situation ................................ 192

Table 5.23: Costs of Renal Failure: the Most Conservative Situation ......................... 193

Table 5.24: Costs of Renal Failure: the Base Case................................................................ 194

Table 5.25 Costs of Glycaemia Control ............................................................................. 195

Table 5.26: Diabetes Costs in Absence of Complications ................................................ 197

Table 5.27: DRGs Used in the Costing ......................................................................................... 198

Table 5.28: Sensitivity Analysis Results: Lifetime Costs \& Harm/Litre Associated

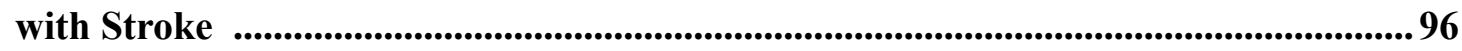

Table 5.29: Sensitivity Analysis Result: Lifetime Costs \& Harm/Litre Associated with IHD ......................................................................................................................................99

Table 5.30: Sensitivity Analysis: Costs Associated with Diabetes ..............................98

Table 6.1: Results of Simulation (Full-Table) .....................................................................204

Table 6.1.1: Results of Simulation (Reduced-Table) ....................................................106 
Table 6.2: Simulated Effects of Taxes Using Estimates from Ni Mhurchu et al.'s..205

Table 6.3: Simulated Effects of Taxes Using Estimates from Sharma et al.'s ..........206 Table 6.4: Simulated Effects of Taxes Using Estimates from Dharmasena \& Capps's 206

Table 6.5: Post-Tax Consumption Using Estimates from Sharma et al.'s .................207 


\section{List of Figures}

Figure 2.1: Channels of Benefits from SSB Taxes........................................................36

Figure 5.1: Markov Chain for a Stroke Survivor ...........................................................82

Figure 5.2: Proportion of Survival Patients after a Stroke .......................................................83

Figure 5.3 Algorithms of Diabetes Interventions .............................................................88

Figure 5.4 Algorithms of Diabetes Treatment \& Changes in Glycaemia Levels ................89

Figure 5.5: Proportion of Survival Patients after a Stroke (Cerebral Haemorrhage Only)

Figure 5.6: Proportion of Survival Patients after IHD Discharge in Dunedin...................174

Figure 5.7: Proportion of Survival Patients after IHD Discharge in Invercargill...........174

Figure 5.8 Stroke Rate by Year Predicted by the UKPDS Model ……...............................196

Figure 5.9 IHD Rate by Year Predicted by the UKPDS Model .............................................196 


\section{Introduction}

\subsection{Background}

Obesity and diabetes today threaten people's quality of life and impose large financial and social costs on societies (Cawley \& Meyerhoefer, 2012; Cox, 2016). Studies have revealed that obesity has become one of the key risk factors for a few epidemics, such as type-2 diabetes, cardiovascular disease, and cancer (Adair et al., 2016). In the USA, the medical costs of obesity alone were estimated to be between $\$ 147$ and $\$ 190$ billion (Cawley \& Meyerhoefer, 2012; Finkelstein et al. 2009). In New Zealand, a conservative estimate of healthcare costs associated with obesity was NZ\$135 million in 1997 and NZ\$624 million in 2006 (Swinburn et al., 1997: Lah et al., 2012).

The prevalence of obesity has been increasing in recent decades, especially in younger age groups. More than 1.9 billion adults were overweight in 2016, and 650 million were obese (World Health Organization, 2020). In New Zealand, data suggest that around $31 \%$ of the adults were obese in 2013 and $10.7 \%$ of children aged 2 to 14 were overweight or obese (Ministry of Health, 2013). In addition, people with lower socioeconomic status are much more likely to be overweight or obese (Adair et al., 2016). This health disparity is clearly seen in New Zealand: Europeans and Asians were advantaged in comparison to Māori and Pacific populations across all socioeconomic indicators (Ministry of Health, 2018). In 2015, 14.7\% and 29.8\% of the Māori and Pacific children aged 2 to 14 years were obese, which was higher than the average level (Ministry of Health, 2013; Cox, 2016).

Type-2 diabetes is a chronic disease characterized by high blood sugar levels (hyperglycemia). It is a result of insufficient insulin production or an ineffective response to the insulin the body produces (Foley et al., 2011). According to the 
Virtual Diabetes Register of New Zealand in 2018, there were approximately 250,000 diabetic patients in New Zealand, and $90 \%$ of these registrations were for type-2 diabetes (Ministry of Health: Virtual Diabetes Register, 2019).

Diabetes is associated with severe long-term consequences. Microvascular consequences include peripheral neuropathy, diabetic retinopathy, and nephropathy. Macrovascular consequences include ischemic cardiovascular disease, stroke and heart failures. The risk of developing these complications can be reduced with good management of blood pressure, blood glucose and blood cholesterol, but increased with diabetes duration (Foley et al., 2011; Best et al., 2012).

Some epidemiologists argue that one of the potential causes of obesity and diabetes could be excess sugar intake (Adair et al., 2016; Parmenter, Jordan \& Jayasinghe, 2017). They suggest that excess sugar intake leads to a higher glycemic level, which can eventually result in obesity, diabetes, and even some fatal diseases, such as cardiovascular disease and pancreatic cancer (Hu, 2013). Several studies have shown that people are currently consuming considerably more sugar than before. For example, the amount of sugar consumed in Mexico in 2006 was as twice high as it was in 1999 (Barquera, 2008), and the US sugar consumption has doubled in the last few decades (Popkin, 2010). In New Zealand, the average sugar consumption was around 158g per day in 2009, which was much higher than the recommended amount of 40g per day (Thornley, Hayden \& Garry, 2010).

Some public health scholars have argued that a major source of sugar is sugar sweetened beverages (SSBs) (Ogden et al. 2011; Andreyeva et al. 2009); other sources include honey, fruits, corn, sauces, salad dressings, and desserts (Azais-Baesco et al., 2017). A 12-ounce portion of SSB usually contains 50g of sugar (Gustavsen \& Rickertsen, 2011).

SSB consumption is substantial. For example, almost $50 \%$ of people drink SSBs on any given day in the US (Ogden et al. 2011). As a result, people in America consumed approximately 45 gallons per person per year in recent years (Andreyeva et al. 2009); daily SSB consumption for children and adults was around 156 and 194 cal 
respectively in 2009 (Smith et al., 2010). In New Zealand, SSBs constituted approximately 5\% of whole household food expenditure in 2010 (Ni Mhurchu et al., 2013). Admittedly, energy is essential to survival and functioning, but it does not have to be sourced from sugar and SSBs. SSBs are high in calories but have little or no nutritional value (Briefel et al., 2009).

The association between intensive SSB consumption and obesity and diabetes has been identified in many studies (Hu, 2013). For example, Ludwig (2001) found that an additional unit of SSB consumption per day increases the risk of developing obesity by $60 \%$. Bawa (2005) found that one can of soda per day is likely to lead to almost $7 \mathrm{~kg}$ of weight gain in 1 year. Schulze et al. (2004) revealed that females whose SSB consumption was increasing had significant weight gain. Also, de Kong et al. (2011) found that, compared with non-SSB drinkers, intensive drinkers have $20 \%$ higher risk of developing diabetes in 24 years.

\subsection{SSB taxes}

Various interventions have been proposed to combat obesity. These interventions include, but are not limited to, SSB taxes, health education, social media campaigns, promotion of physical activities at and after school, improvement in availability of healthy food at schools and in workplaces, etc. (Bleich et al., 2013; Burgess et al., 2017). These interventions either try to reduce SSB consumption or increase people's energy expenditure, thereby achieving weight-loss. Obesity is one of the key risk factors for type 2 diabetes and cardiovascular disease. If such factors are controlled well, an improvement in health outcomes would be likely in the long run (Adair et al., 2016).

Scholars have claimed that a single intervention alone is unlikely to achieve a desirable goal. For instance, Jou et al. found that a social media campaign alone is insufficient (Jou et al. 2014). Some systematic reviews have shown that a mix of different interventions may yield desirable effects, but the evidence is somewhat weak (Bleich et al., 2013; Burgess et al., 2017). Also, Wilson and Hogan (2017) argued that 
regulations on branded cool packaging, clear and prominent calorie content labelling, and limits to convenience, probably also have great impacts.

SSB taxes have been applied as an intervention, with the expectation that such taxes might increase the price of SSBs, and thereby reduce SSB consumption. For example, in America, 24 states and six cities have passed SSB taxes since 2009. Other countries, such as the UK, Mexico, Chile, Finland, Hungary, and Ireland, have all imposed taxes on SSBs (Friedman \& Brownell, 2012).

Some scholars have suggested that the reasons governments favor taxes are that the costs of implementation for such taxes are relatively low, and the taxes may be paid by a broad spectrum of the population, thereby generating considerable tax revenues (Andreyeva et al. 2009; Powell \& Chaloupka, 2009; Friedman \& Brownell, 2012). This argument is supported by a study conducted by Friedman and Brownell (Friedman \& Brownell, 2012). They claimed that in the US states of Mississippi, Louisiana, and West Virginia, a penny-per-ounce excise tax on SSBs could generate \$136 million, \$210 million, and \$84 million respectively in the year 2013 (Friedman \& Brownell, 2012). Some advocates for corrective taxes suggest that the tax revenue might further be used for public health campaigns and subsidies on healthcare services (Brownell et al., 2009). The benefits (tax revenue and health improvement) could be larger amongst people who have high SSB consumption, as those people are more likely to be overweight and more responsive to taxes (Powell \& Chaloupka, 2009).

Possibly due to the advantages discussed above, SSB taxes are supported by the public in New Zealand. A New Zealand Herald poll indicated that $83 \%$ of 11,700 respondents support a sugary beverage tax (Herald on Sunday, 2016). Other surveys indicate that SSB taxes are more likely to be supported by the public when the tax revenues would be used for health promotion for key groups such as children and people with low socioeconomic status (Jacobson \& Brownell, 2000).

SSB taxes are also supported by some health experts in New Zealand. For example, health researcher Dr Gerhard Sundborn said: 
"A sugary drinks tax is the most effective means to address this (obesity and dental problems), and only the Government can enact it. It requires the Government to provide leadership which prioritizes our children's health over corporate profits." (Herald on Sunday, 2017)

The support of SSB taxes from public and some experts is probably from quite a few studies which indicate that consumption and energy intake will be reduced if such taxes are imposed. Andreyeva et al. estimated that a penny-per-ounce tax could reduce SSB consumption by $24 \%$, which could ultimately reduce per-capita caloric intake by 145-150 calories per day (Andreyeva et al. 2009). Ni Mhurchu et al. argued that a $20 \%$ SSB tax could be an effective strategy to deal with the high burden of diet-related diseases in New Zealand (Ni Mhurchu et al., 2013). Colchero et al. found that a $10 \%$ increase in price reduced SSB consumption by $6 \%$ in US, which was equivalent to $-12 \mathrm{~mL} /$ capita / day (Colchero et al., 2016). Cornelson et al. revealed that a tax of $£ 0.10$ per-beverage decreased individual consumption by $11 \%$ in the UK (Cornelson, 2017).

However, the evidence that SSB taxes can reduce obesity is quite weak (Wilson and Hogan, 2017). A short period study from Mexico indicated that SSB taxes failed to decrease the obesity rate. In fact, the rate continued to increase after such taxes were imposed (Colchero et al., 2016). The challenge is that health benefits generated by such taxes cannot be captured in a short period; and the sugar or SSB taxes that have already been imposed in real world have not been in place that long enough (Wilson and Hogan, 2017). In addition, the casual link between SSB taxes and obesity is a complicated one with at least four steps. First, an increase in prices must be seen after such a tax is imposed. Second, such an increase in price has to result in a decrease in consumption. Third, the reduction in consumption must decrease people's sugar intake. Finally, the decrease in energy intake has to lower risk factors (e.g., obesity) (Wilson and Hogan, 2017). The complexity of the real world and of human behaviors ensures that none of these steps is a deterministic one. 
Many SSB taxes fail at the first or second step. Studies report that reductions in consumption are too small to generate a substantial decrease in energy intake. Even when there is a reduction in SSB consumption, the impact on obesity can be undermined by substitution of other untaxed sugary goods like fruit juices and high fat milk (Fletcher et al., 2010; Duffey et al., 2010). For example, Fletcher et al. (2010) estimated that in the US, the effects of SSBs taxes were completely offset by substitution of untaxed goods. Hence the real health benefits of SSBs taxes could be much lower than expected (Duffey et al., 2010; Fletcher et al., 2010).

Given the arguments discussed above, the New Zealand Beverage Council (NZBC) did not support the introduction of a sugar tax (NZBC, 2020). Instead, they advocated for a mixed of diet education and physical activity promotion (NZBC, 2020). As a result, a sugar tax was not included in the government's Childhood Obesity Plan in 2015 (Ministry of Health, 2017).

However, a justification for a corrective tax should be based on externalities or another market failure, rather than the effects of SSB taxes (Brownell et al., 2009). When private costs and benefits deviate from social costs and benefits, then an externality occurs, and equilibrium outcome will not be optimal (Buchanan, 1962). When the health and welfare system is publicly funded, patients usually do not have to bear the full costs of healthcare services. Hence the full costs of SSB consumption do not completely pass to consumers who develop those diseases associated with excess SSBs intake but fall on all those whose taxes are used to fund the delivery of healthcare services (Brownell et al., 2009). As a result, consumption of SSBs is associated with externalities.

An internality is another type of market failure. It arises when there is a long-term personal cost to people that they do not fully account for when making consumption decisions (Marron, 2015). One potential cause of this issue is hyperbolic discounting, which occurs when people assign a higher marginal discount rate to costs and benefits in the immediate term, but a lower rate over a longer period (Robinson, 2011). For example, a consumer does not plan to drink a SSB tomorrow because he or she values 
the enjoyment from the SSB less than the costs of future health and health-related consequences. However, when tomorrow arrives, this person switches to drinking the SSB. In this situation, a self-control problem occurs and the harm of current SSB consumption is undervalued.

Alternatively, consumers may not have complete information to make optimal decisions (Finkelstein, Ruhm, \& Kosa, 2005). For instance, most people cannot accurately estimate the number of calories they consume, especially when they eat out. Admittedly, this problem probably could be solved partly by a nutrition labelling regulation and a health education programme of calorie counting (Smith, Chouinard \& Wandschneider, 2011). However, even if the energy content is labelled in every $\mathrm{SSB}$, and people apply calorie counting, it is still not possible for them to know what is appropriate for each person's individual biology, e.g., for their metabolic system. Human biology is complex and dynamic with billions of cells and multiple organ systems that are constantly changing with interactions with the external environment that are voluntary (Sgarbieri \& Pacheco, 2017). We cannot figure out all these interactions and their impact because much of them are unobservable and occur at a cellular level. As a result, the full impact of consumption of SSBs is impossible to know and consumers may not behave in their best interest.

Furthermore, consumers are probably not always rational and utility maximizing (Samson, 2014). Their behaviors can be influenced by unconscious factors, personality traits, individual experiences, reference groups (e.g., family members and friends), and physiological or emotional states. Physiological or emotional states can be easily affected by marketing strategies which remind them of happy memories or touches and feelings through TV advertisements and product designs. This is particularly true for SSBs as they are often heavily marketed. As a result, consumers may purchase SSBs impulsively (Samson, 2014).

Because of both externalities and internalities, SSB consumption in market equilibrium may be above the socially optimal level, resulting in a dead-weight loss (costs of market inefficiency) to society. Thus, interventions may be necessary to 
decrease demand or supply, ideally getting the consumption close to the social-optimal level and increasing social welfare.

\subsection{Statement of the Problem}

Probably because the WHO advocates for at least a $20 \%$ SSB tax to prevent non-communicable diseases, a $20 \%$ ad valorem tax has been imposed in a few countries (Lal et al., 2017; Elite \& Sharma et al., 2014; Briggs et al., 2013). However, there have been a number of studies looked at a tax level of $20 \%$, but none of them discussed whether the $20 \%$ tax rate, or another tax rate, is at the socially optimal level or not. This is important because a tax is efficient only when it maximizes the welfare of the whole society (Allcott, Lockwood, and Taubinsky, 2019). In addition, as discussed previously, one of the potential reasons why a SSB tax may fail to reach its policy goals is that the tax rate is not high enough to cause a substantial decrease in SSB consumption and energy intake. Given these arguments, it is natural to ask:

(1) What is the socially optimal level for SSB taxes?

In addition, as government is assumed to choose a tax rate to maximize social welfare given a tax base (by litres, prices, or calories), it would also be natural to ask:

(2) Which tax base is the most efficient? If sugar or calories is the harmful ingredient, is a tax per calorie superior to other tax bases?

As discussed previously, SSB taxes may not be effective, as consumers may switch to other sugary foods which are plausibly substitutes for SSBs. Given this possibility, it is worth studying two additional questions:

(3) Are SSB taxes still beneficial to society when both substitutes and complements are accounted for?

(4) Is it better to tax all sugary drinks, or just the ones classified as SSBs?

\subsection{Research Method}

(1) Socially optimal level 
Research question 1 concerns the level for a socially optimal tax on SSBs. To answer this question, a simple theoretical model is constructed, in which a representative consumer chooses how much SSBs to drink. The consumption choice does not account for externalities, and only accounts for some of the future health consequences to the drinker (i.e., the consumer is subject to internalities). This simple model follows previous studies of optimal taxes on sin goods, in which the optimal rate for the corrective tax is commensurate with the sum of externalities and internalities. Then, an extended model is introduced, which incorporates different commodities and allows substitution and complementary effects. In addition, the model is extended to allow for a range of different tax bases.

The optimal corrective tax formula can be derived from the extended model described above. The optimal corrective tax formula includes three terms: (i) the proportion of health harm that is not reflected in consumption, (ii) the marginal harm of consumption associated with externalities, and (iii) a scaler for the tax which reflects the effects of substitutes and complements. In order to estimate the socially optimal tax rate, the three terms in the formula have to be measured.

In order to measure the proportion of health harm that is not reflected in consumption, contingent valuation $(\mathrm{CV})$ is then applied in this study. Compared with cost-utility analysis (CUA), in which health outcomes are usually assessed by quality-adjusted life years (QALYs) or disability-adjusted life years (DALYs), outcomes valued in CV or CBA research usually include not only health consequences, but also non-health outcomes, such as income loss and time costs (Smith and Sach, 2009). Given that excessive SSB consumption may increase health risks in the long run, we decided to conduct an online CV survey on Facebook to measure people's willingness-to-pay (WTP) for health risk changes, and thereby further estimating the proportion of health harm that is not reflected in consumption.

In order to estimate the marginal harm of consumption associated with externalities, a cost analysis using a Markov model and the UK Prospective Diabetes Study (UKPDS) model is conducted to measure the lifetime costs for the diseases associated with 
excessive SSB consumption. This provides estimates of the marginal harm from SSBs associated with externalities.

In order to measure the scaler for the tax which reflects the effects of substitutes and complements, data reported in Ni Mhurchu et al.'s study (2012) are used in the base case which demonstrates the scenarios in New Zealand. Our study also reports calibration exercises using the Australian information from Sharma et al. (2014) and American data from Dharmasena and Capp's (2011).

(2) Tax Base

In order to answer which tax base is the most effective, a welfare measurement is developed to evaluate the welfare change due to taxes. This is necessary because the optimal tax rate does not tell us the magnitude of the welfare gain resulting from imposing such a tax. We assumed the simplest function form, quadratic and quasi-linear utility function, which guarantees that marginal benefits will be linear. In addition, the approach is equivalent to using a multi-commodity deadweight loss measure, with linear approximations of demand curves.

In order to investigate the best tax base, welfare at its optimal tax level is compared for each tax base. The tax base which generates the highest post-tax welfare is the most effective option. As with the socially optimal tax rate, the calibration uses New Zealand data from Ni Mhurchu et al. (2012), the Australian information from Sharma et al. (2014), and American data from Dharmasena and Capp's (2011).

(3) SSB taxes, sugary beverage taxes, or no tax?

To answer research questions 3 and 4, the welfare measurement derived in (2) is used to evaluate the welfare change due to different taxes. In order to explore whether SSB taxes and beverage taxes are beneficial to society, the post-tax welfare is compared with the initial welfare where no taxes are imposed. If the post-tax welfare is higher than the initial welfare, then taxes will be beneficial to society, and vice versa.

Finally, to investigate whether taxing all sugary beverages is better than taxing SSBs only, the highest post-tax welfare generated by a SSB tax at its optimal tax level is 
compared with the highest post-tax welfare generated by a beverage tax at its optimal tax level. The one which yields a higher welfare is the superior option.

\subsection{Thesis outline}

This thesis is comprised of seven chapters. Chapter two is a systematic review of the literature on SSB taxes. Chapter three presents the utility-maximization model and uses it to investigate the socially optimal levels of SSB taxes and the welfare change associated with such taxes. Chapter four discusses a CV study, the result of which is used to measure the proportion of health harm that is not reflected in consumption. In chapter five, a cost analysis is conducted to estimate the marginal harm of consumption associated with externalities. In chapter six, the utility-maximization model is simulated using the findings of the CV study and the cost analysis, as well as values reported in other studies. Chapter seven is a summary of the whole thesis and policy implications are discussed.

\subsection{Conclusion}

In this chapter, the background information of this research is introduced, and the main five research questions are identified. Obesity and diabetes today threaten people's quality of life and imposes large financial and social costs on societies. Therefore, SSB taxes have been applied as an intervention against obesity and diabetes. A justification for such a corrective tax is based on market failure, for example, externalities and internalities.

There is a gap between which tax base is the most efficient, and whether the $20 \%$ tax rate, or another tax rate, is socially optimal or not. Thus, the first two research topics in this thesis concern the socially optimal tax rate and the best tax base. In addition, given that consumers may switch to other untaxed sugary foods which would be substitutes for SSBs, there is a need to investigate two additional questions in this thesis, they are: (1) Are SSB taxes still beneficial to consumers and society, when both substitutes and complements are accounted for? And (2) is it better to tax all sugary drinks, or just the ones classified as SSBs? 
Having identified these research questions, the methods used to answer these questions are described, followed by a description of the thesis outline. 


\section{A Review of the Literature on SSB Taxes}

\subsection{Introduction}

This chapter reviews previous studies which examined the research questions listed in the previous chapter. In detail, we reviewed the literature to identify publications which (1) described those SSB taxes which have been imposed in the world, (2) explored the effects of SSB taxes, (3) investigated the socially-optimal level of SSB taxes, (4) compared different tax bases, (5) identified the benefits and costs of SSB taxes, (6) measured internalities and externalities associated with SSB consumption, (7) estimated elasticities of SSBs, and (8) compared SSB taxes with more general beverage taxes.

\subsection{Search Strategy \& Selection}

A systematic review is a rigorous methodology for synthesizing findings of previous studies, by applying transparent, explicit, and replicable procedures (Khan et al., 2011). This process includes a clear search strategy, inclusion or exclusion criteria, data extraction and reporting (Langer and Stewart, 2014). Following this method, details of the search strategy and selection process of this literature review are discussed below.

Studies up to June $7^{\text {th }}, 2019$, were searched for in Medline (from 1994), EconLit (from 1800), Scopus, and Web of Science (from 1864), as well as the Cochrane Database of Systematic Reviews (from 1999). Key phrases searched included "sweetened soda beverages tax", "sugar sweetened beverages tax", "soft drink tax", "soda tax", "soda beverages levy", and "soda levy". Only studies written in English were included. The search initially yielded more than 3,900 results (including duplicates). Since there were too many papers to review, a selection had to be made. The following selection criteria were used to narrow the search: 1) studies from 
January $1^{\text {st }}, 2013$, onwards; 2) duplicates were excluded; 3) the title of the study indicated that it examined the effects of SSB consumption on the whole population, rather than only a sub-group (e.g., children or adolescents. This is thought to be reasonable because it is almost impossible to tax a specific sub-group); 4) the whole article was available rather than just an abstract; 5) the title or abstract showed that the focus of the study dealt with elasticity, SSB substitutes, income heterogeneity, tax base, pass-through rate, cost-benefit analysis, cost-effectiveness/utility analysis, healthcare costs, tax revenue, effects on diabetes, cardiovascular disease, and obesity; 6) a quantitative approach was taken in the study; 7) systematic reviews and meta-analyses were excluded. This effort finally yielded 37 studies. Details are discussed below.

\subsection{Search Results}

SSB taxes or sugar-added beverage taxes that have been imposed in the real world before 2019 have been well summarized by Cawley et al. (2019). According to this paper, SSB taxes had been applied in seven U.S. cities and 33 countries before 2019 (Cawley et al., 2019; GFRP, 2019). ${ }^{1}$ In general, tax rates vary from being very low (i.e., 1 cent per ounce) to substantial (100\% per bottle) amongst the selected studies. Ad valorem taxes are the most used type of taxes, the rates of which vary from $1 \%$ to $100 \%$. It should be noted that milk or fruit-based drinks are exempt in many countries such as Barbados, Brunei, and Ireland. Detailed information is reported in the supplementary material in Cawley et al. (2019). ${ }^{2}$

In the previous literature, the following topics have been investigated: cost-effectiveness of a SSB tax, SSB tax bases, the effects of SSB taxes on healthcare costs, tax revenue, elasticities or effects on SSB consumption, energy intake, health and disease (obesity, diabetes, and cardiovascular diseases), pass-through rates of SSB taxes; the effects of an SSB tax on substitutes, internalities and externalities of SSB consumption, and the socially optimal tax. No study was found which investigated

\footnotetext{
1 Countries where SSB taxes had been applied before 2019: Bahrain, Barbados, Belgium, Brunei, Chile, Dominican, Fiji, Finland, France, French Polynesia, Hungary, India, Ireland, Kiribati, Mauritius, Mexico, Norway, Palau, Peru, Philippines, Portugal, St Helena, Samoa, Saudi Arabia, South Africa, Spain, Thailand, Tonga, United Arab Emirates, United Kingdom, some sates in the United States, and Vanuatu (Cawley et al., 2019).

2 https://doi.org/10.1146/annurev-nutr-082018-124603
} 
whether sugary-drink taxes are superior to SSB taxes. Details of the findings are synthesized below.

\subsection{Socially Optimal Level}

What is the socially optimal level of a tax? According to Pigou (1920), the optimal corrective tax should equal the sum of the internalities and externalities of marginal consumers. Amongst the selected literature, numbers of studies looked at a tax level of $20 \%$. However, none of them discussed whether the $20 \%$ tax rate, or another tax rate, is at the socially optimal level or not. One possible explanation is that the WHO advocates for at least a $20 \%$ SSB tax, to prevent non-communicable diseases (Lal et al., 2017; Elite \& Sharma et al., 2014; Briggs et al., 2013).

The socially optimal tax rate has been investigated in three selected studies. Although the main focuses of Marron (2015) are to compare taxation of externalities with internalities, and to address equity concerns, the formula for the socially optimal tax rate is derived using a utility maximization model in this study. He starts from a utility function which includes a sin good and a non-sin good. The consumption of the harmful product yields health harm and externalities. Consumers are assumed to account for a proportion of health harm due to time inconsistency and lack of self-control, but do not account for any externalities. In addition, the sin good is assumed to be produced at a constant marginal cost in a perfect competitive market so that price is equal to the marginal cost and average cost. In order to address over-consumption, government imposes a sin tax which will fully pass to consumers. The consumer's budget is constrained by income including a lump sum rebate from the corrective tax. Having substituted the budget constraint into the utility function and then solved the utility-maximization equation, the formula for a socially optimal tax level is derived. The familiar result is that the corrective tax should be set equal to the proportion of the marginal harm that the consumer does not account for. The result is a generalization of Pigou's account of optimal corrective taxes.

Griffith et al. (2018) formulate an optimal tax formula using a similar approach, but they exclude externalities in their study, because their focus is on correcting internalities only. Unlike Marron (2015), their model includes a number of consumers, 
and they explicitly identified the utility is quasilinear which is in the form $\mathrm{U}(\mathrm{x}, \mathrm{y})=$ $\mathrm{u}(\mathrm{x})+\mathrm{z}$. This set up guarantees that the consumption of $\mathrm{x}$ is independent of income, and the marginal utility of $\mathrm{z}$ which can be regarded as income, is constant. Having substituted the budget constraint to the utility function and then solved the utility-maximization problem, the formula for the socially optimal tax level is derived. The results indicate that the optimal tax equals the sum of average internalities plus an adjustment based on the covariance of internalities and the slope of demands.

The two studies described above mathematically justified Pigou (1920)'s argument that the optimal corrective tax should equal the sum of the internalities and externalities. However, neither of these studies incorporates substitutes and complements in their optimal tax approach. If consumers switch from a sin good to other untaxed harmful products, the benefits of such taxes will probably be overstated. Given this, the socially optimal level with consideration of substitution may differ from the level when substitution is not accounted for.

The only study that addresses the socially optimal level of an ad valorem tax with substitutes and complements is Allcott, Lockwood, and Taubinsky (2019). Diet drinks, alcohol and cigarettes are included as substitutes, because they think consumers may treat them as SSBs as classes of tempting pleasures. As with Marron (2015) and Griffith (2018), Allcott, Lockwood, and Taubinsky's (2019) also construct a utility-maximization model to investigate the socially optimal level, although they concentrate on equity and redistribution. Results demonstrate that the optimal tax formula includes two parts: (1) the benefits which represent the welfare increased from reducing internalities, and (2) the regressivity costs which represent the social loss of transferring welfare from poorer to richer. Allcott, Lockwood, and Taubinsky (2019) conclude that sin tax regressivity can be offset by targeted transfers or income tax reforms, if differences in consumption are due to income effects, rather than preference heterogeneity. In addition, their results indicate that the optimal tax level may be slightly higher than current SSB taxes in US (1 to 2 cent per ounce) (Allcott, Lockwood, and Taubinsky, 2019). My main concern about this study is that cigarettes are probably not a substitute for SSBs. This argument is supported by the finding that 
the cross-price elasticity of SSBs and cigarettes is statistically insignificant (Allcott, Lockwood, and Taubinsky, 2019). One plausible explanation is that people consume SSBs not because SSBs are classes of tempting pleasures. Instead, SSBs are purchased simply because people are thirsty. Therefore, other beverages such as milk drinks, fruit drinks, and energy drinks should probably be included, and cigarette should be excluded. Consequently, the substitution effects and optimal tax rate estimated by Allcott, Lockwood, and Taubinsky (2019) are possibly biased.

\subsection{Comparison of Tax Bases}

For the excise taxes in the selected studies, the tax format could be X cents /ounce of $\mathrm{SSB}$ or sugar, X cents/litre of SSB, X cents/calorie, or X cents/bottle, or Ad valorem taxes (tax by price, $\mathrm{X}$ cents $/ \$$ ).

Nutrient taxes have been found to be more effective in decreasing energy intake (Zhen, Brissette \& Ruff, 2014). Zhen, Brissette \& Ruff have developed a modified distance metric model of differentiated product demand which endogenizes cross-price effects, to consider substitutes and complements. Based on the Almost Ideal Demand System (AIDS) estimation, their simulation suggested that to reduce 3,500 calories from beverage consumption, a calorie-based beverage tax would cost $\$ 1.40$ less in compensating variation than an ounce-based tax, which indicates that taxing by sugar is better than taxing by ounce (Zhen, Brissette \& Ruff, 2014). The main concern with their study is that the different taxes are not compared at their socially optimal levels. As a result, it is quite hard to conclude which tax base is the most efficient.

Different tax bases are also compared by Grummon et al. (2019). They start by deriving mathematical expressions for the effects of volumetric taxes and sugar taxes. Full pass-through of taxes (the entire tax is passed to consumers) is assumed in this study. As with Griffith et al. (2015), the utility function is assumed to have a quasilinear form. Having defined 'economically equivalent taxes' as those that impose the same tax rate on all SSBs with average sugar content, Grummon et al. (2019) demonstrate that when calorie taxes and volumetric taxes yield the same post-tax consumption, calorie taxes reduce more sugar intake and consequently generate a larger economic efficiency gain. A potential weakness of this study is the 
"economically equivalent taxes" assumption. We believe there is no reason to keep the tax rates "economically equivalent" when tax base is changed. Instead, the best tax rate should be imposed, given the tax base the government has. In addition, this study can be further extended by (1) comparing different tax bases (including ad valorem taxes, the most commonly used tax base in real world) at their optimal levels, (2) allowing for the effects of complements and substitutes, and (3) adding a serious measurement of externalities and internalities.

Finally, Wang (2014) simulate the effects of both a penny per ounce tax and a sales tax. They build a utility maximization model and demonstrate that a penny per ounce tax results in a larger impact on consumption than the sale tax. As with Zhen, Brissette \& Ruff, the welfare change associated with taxes is calculated in terms of compensating variation. Given that a per ounce tax yielded a smaller compensating variation, this study concludes that a penny per ounce is superior to the sales tax. As with the studies discussed, the main concern with this study is that different tax bases are not compared at their socially optimal levels. As a result, it is hard to determine which tax base is the most efficient.

In summary, different tax bases have been investigated by a few studies. However, there is gap to compare different tax bases at their socially optimal level without the “economically equivalent" assumption. A potential contribution could be achieved by using the optimal tax formula derived by Allcott, Lockwood, and Taubinsky (2019), including different tax bases but excluding equity concerns.

In addition, it should be noted that for most theoretical studies in which a utility-maximization model is built, consumers are usually assumed not to vary in their marginal utility of wealth, and taxes do not have income effects so that demand is locally linear (Griffith et al., 2018; Grummon et al., 2019; Wang, 2014).

\subsection{Effects of SSB Taxes}

The effects of SSB taxes have been investigated in several empirical studies. Some studies found SSB taxes to be effective in reducing consumption, while others did not. For example, Colchero et al. found that a $10 \%$ increase in price reduced SSB consumption by $6 \%$ in the US, which was equivalent to a reduction of 12 
$\mathrm{mL} / \mathrm{capita} /$ day (Colchero et al., 2016). Cornelson et al. reported that a tax of $£ 0.10$ per beverage decreased individual SSB consumption by $11 \%$ in the UK (Cornelson, 2017). Silver et al. (2017) found that a $20 \%$ increase in price could reduce energy intake per person by $45 \mathrm{kcal}$ per day in Berkeley, and $131 \mathrm{kcal}$ per day nationally. The substantial difference between Berkeley and other places is that it is quite easy for people in Berkeley to go to their neighboring counties where no SSB taxes are imposed, as Berkeley is a small county.

It should be noted that a decrease in SSB consumption could be associated with an increase in diet soft drink consumption and other untaxed beverages, due to a substitution effect (Jue et al. 2012; Dharmasena \& Capps 2012; Dharmasena et al. 2014). Colchero et al. found that there was an increased demand for pure water and fruit drinks after a SSB tax was imposed in Mexico (Colchero et al., 2017). They pointed out that the total effects of SSB taxes on calories should be questioned, as the consumption of fruit drinks went up. Consequently, calorie intake decreased less than 0.5 percent. Furthermore, the SSB taxes in Mexico only had a short-lived effect. SSB sales decreased from 16,375 million litres in 2013 to 15,915 million litres in 2014, and then rebounded to 16,156 million litres in 2016. A potential explanation for this phenomenon could be that consumers had become used to the post-tax prices. Given this, it is not a surprise to see that SSB taxes failed to decrease the obesity rate. The obesity rate actually continued to increase after such taxes were imposed (Colchero et al., 2016).

Other studies also suggest SSB taxes may not be effective, due to the increase in energy intake from non-taxed goods. For example, Fletcher (2010) reported that a 1\% increase in SSB taxes would raise caloric intake from non-soda beverages by 7.5 calories, which might offset the effects of decreased SSB consumption (Fletcher et al., 2010). People in Berkeley consumed more non-taxed products, such as milkshakes and fruit juices which may even contain as much, or even more calories than SSBs (Cawley et al., 2015; Falbe, 2015 et al.; Silver et al., 2017).

Scholars have argued that SSB taxes may be ineffective when consumers fail to notice the price increase caused by the taxes (Colantuoni et al. (a), 2015; Colantuoni et al. 
(b), 2014). This possibility is suggested by apparently ineffective taxes set at lower rates. In both studies in which SSB taxes were ineffective, the imposed tax rates were 5\% (Colantuoni et al. (a), 2015; Colantuoni et al. (b), 2014). But in other studies where SSB taxes were effective, the tax rates were $10 \%, 20 \%$, or even higher (Colchero et al. (b), 2016; Falbe et al., 2015; Fletcher et al., 2015; Silver et al., 2017; Waterlander et al., 2014).

This argument is also supported by an experimental study. Bollard et al. (2016) conducted an experiment in New Zealand to assess the impacts of a $20 \%$ ad valorem tax, warning labels, and plain packaging on SSB consumption in 604 young consumers aged 13 to 24 who identified themselves as regular SSB consumers in an online survey in 2014. Participants were randomly allocated to be exposed to one of 12 experimental conditions generated from a computer algorithm. The 12 conditions were combined from an image of branded or plain packaged beverages, with or without a $20 \%$ ad valorem tax, and with either without any warning, a text warning, or a picture warning. Given one of the specific conditions, participants were asked to show the probability of purchasing using seven-point Likert scales. Results showed that the decrease in purchase probability associated with a $20 \%$ ad valorem tax was insignificant. This is probably because beverage prices were not displayed prominently enough, and the absence of non-taxed comparators (Bollard et al., 2016). In addition, the generalizability of the findings was probably limited by convenience sampling method and low response rates from Māori and Pacific (Bollard et al., 2016).

Pass-through rate is the other factor which makes taxes ineffective. Among the selected studies, only one study found the tax was over-shifted to consumers (Cawley and Frisvold, 2016); others all suggested that pass-through rates varied from around $50 \%$ to $100 \%$ (Falbe et al.; 2015). Given the low tax rates and under-shifted pass-through ratios, it should not be very surprising to see in some studies SSB taxes did not have a significant impact on price and consumption (Cawley and Frisvold, 2016; Falbe et al., 2015; Colantuoni, 2014).

The common challenge for those empirical studies is that how to identify the effects 
of SSBs in the presence of noise and confounders. The ideal approach would be a randomized controlled trial (RCT), which is widely accepted as the gold standard for quantitative studies (Woolcock, Bamberger and Rao, 2010). This method is also recommended as the most reliable method to determine effectiveness (Woolcock, Bamberger and Rao, 2010). However, a RCT is rarely possible in complex real environments. Its feasibility might be constrained by the safety, economic, and geographical conditions of the country (Woolcock, Bamberger and Rao, 2010). Probably because of this, a RCT was applied in only one of the selected empirical studies (Waterland et al., 2014).

Some scholars have argued that when an RCT is not feasible, convenience sampling with a controlled group and the difference-in-difference (DID) method will probably be the next best option (Woolcock, Bamberger and Rao, 2010). From this perspective, the results of the one RCT study and the other studies which applied a DID method may be considered somewhat compelling. In addition, an instrumental variable and a regression discontinuity design probably can be used as feasible and reliable alternative methods to determine causation. But in the selected studies, these methods were seldom applied.

Another challenge identified in estimating structural equations could be that prices and expenditures would be endogenous if there is unobserved demand heterogeneity or local demand shocks (Harding and Lovenheim, 2017). This issue matters because demand heterogeneity or local demand shocks are plausibly correlated with local prices or expenditures (Harding and Lovenheim, 2017). In order to solve this problem, finding instruments associated with prices and expenditures, but uncorrelated with unobserved demand heterogeneity and shocks, is usually required. One plausible instrument suggested in one of the selected studies is the average price of each product across other markets, if the market pricing factors are independent across markets (Harding \& Lovenheim, 2017). As discussed previously, another possible approach to isolate quasi-random variation in non-experimental data is to try to control for product quality and demand fluctuations.

\subsection{Elasticities of SSBs}


The magnitude of the effect of a SSB tax on quantities is usually reported in terms of elasticities of demand. The lack of effectiveness in reducing consumption could also be a result of a low elasticity of demand. There is a wide range of estimates of price elasticities for SSBs. Several papers in the public health literature have sought to estimate the price elasticity of demand for SSBs. Estimated own price elasticities of SSBs varied from -3.91 to -0.48 in the twelve selected studies; cross-price elasticities varied between 0 and 0.64 were reported for substitutes (Ni Mhurchu et al., 2013; Bonnet \& Vincent, 2012; Dharmasena \& Capps, 2012; Kifer, 2015; Zhen et al. (b), 2013; Tiffin et al., 2015; Wang et al., 2015; Schwendicke et al., 2017). A recent study by Wang (2015) estimated the own price elasticity of regular (i.e., non-diet) soda to be -0.6 on average. The author argued that previous estimates of soft drink price elasticities had been upwardly biased (in absolute terms), due to their failure to properly account for stock-piling behavior. All those studies generally reported that SSBs are not very elastic.

In addition, there may be heterogeneity between countries. Ni Mhurchu et al. estimated the own price elasticity of carbonated soft drink and energy drink is -1.27 and -1.34 respectively in New Zealand (Ni Mhurchu et al., 2013). SSBs in France seem to be more elastic: their elasticities varied from -2 to -4 , but they were found to be between 0 and -2 in UK, USA, and Australia (Bonnet \& Vincent, 2012; Dharmasena \& Capps, 2012; Kifer, 2015; Zhen et al. (b), 2013; Tiffin et al., 2015; Wang et al., 2015; Schwendicke et al., 2017). Given the selected studies were all conducted in a similar period and analyzed using similar approaches, the difference may be a result of heterogeneity between countries, or heterogeneity between characteristics of the samples.

The magnitude of substitute effects can be revealed by cross-price elasticity. Ni Mhurchu et al. (2013) have shown that non-alcoholic beverages are the substitutes for energy drinks in New Zealand by estimating cross-price elasticities. Similarly, cordial, fruit drinks, diet soft drinks, water, milk (including milk drinks), tea, and coffee are identified as substitutes for regular soda in Australia (Sharma et al., 2014).

In many studies, people with lower socioeconomic status were more elastic to price 
change compared to those with higher socioeconomic status. This argument appears to have been upheld in this review. Amongst the selected studies, the average own-price elasticity was -0.738 and -0.582 for the low-income groups and middle- or high-income groups respectively (Zhen et al. (b), 2013). In addition, the average own-price elasticity was found to be $-1.288,-0.62$, and -0.45 for heavy, moderate, and light consumers respectively (Tiffin et al., 2015). In New Zealand, the own price elasticity of carbonated energy drink was -7.92 for Maori, and -1.04 for non-Maori non-Pacific (Ni Mhurchu, et al., 2013). Consequently, poorer consumers seem to reduce more SSB consumption and energy intake than their richer counterparts; intensive consumers seem to reduce more SSB consumption and energy intake than other consumers. These predictions are supported in four of the selected simulation studies (Zhen et al. (b), 2013; Tiffin et al., 2015; Wang et al., 2015; Schwendicke et al., 2017).

Of the estimation studies, the AIDS approach is the most commonly used to derive elasticities. An advantage of this method is that the standard errors will be comparatively low, if many goods are estimated (Meyer et al., 2011). The model can be further improved with more flexibility by modifying it to a logit or a quadratic format (Bonnet \& Vincent, 2012; Dharmasena \& Capps, 2012). When there are significant asymmetries in SSB purchasing habits (e.g., a small number of households account for large amount SSB consumption), changing the model to a hierarchical Bayesian structure is usually required (Kifer, 2015). By applying this modification, both within-unit behaviors and cross-section heterogeneities can be accounted for (Kifer, 2015).

In addition, cross price elasticities can be critical for SSB taxes. Creedy (2016) built a simple theoretical model which included three goods, two of which had high calorie contents; the other had low calorie content. The results showed that the effectiveness of a sugar tax can be examined using one own-price elasticity, one cross-price elasticity, relative budget shares, relative prices, and relative calorie contents.

Finally, Gibson and Kim argued that own-price elasticity will probably be overestimated if people's preferences are not well controlled and separated from the 
price response (Gibson \& Kim, 2013). This is probably because consumers may switch to lower quality goods as a response to taxes. They looked at several studies and found the mistake in more than $80 \%$ of the studies (Gibson \$ Kim, 2013).

\subsection{Benefits of SSB Taxes, Internalities, \& Externalities of SSBs}

\subsubsection{Benefits of SSB Taxes}

Benefits of SSB taxes have been identified and investigated in several studies. Among the selected studies, reported benefits of SSB taxes included tax revenue, reduction in health risks, and decreased healthcare costs. A decrease in health risks was simulated in fifteen studies; an increase in quality of life was measured by quality-adjusted life years (QALYs) or disability adjusted life years (DALYs) in five studies; effects on healthcare costs were estimated in eight studies; and tax revenue was simulated in five studies.

The health benefits from decreased SSB consumption can be modelled through the channels shown in Figure 2.1 below. In general, decreased SSB consumption reduces people's energy intake. This is likely to decreases people's body weight, thereby reducing type-2 diabetes and cardiovascular disease risk. This process can be simulated using the specific disease models described in the methods section.

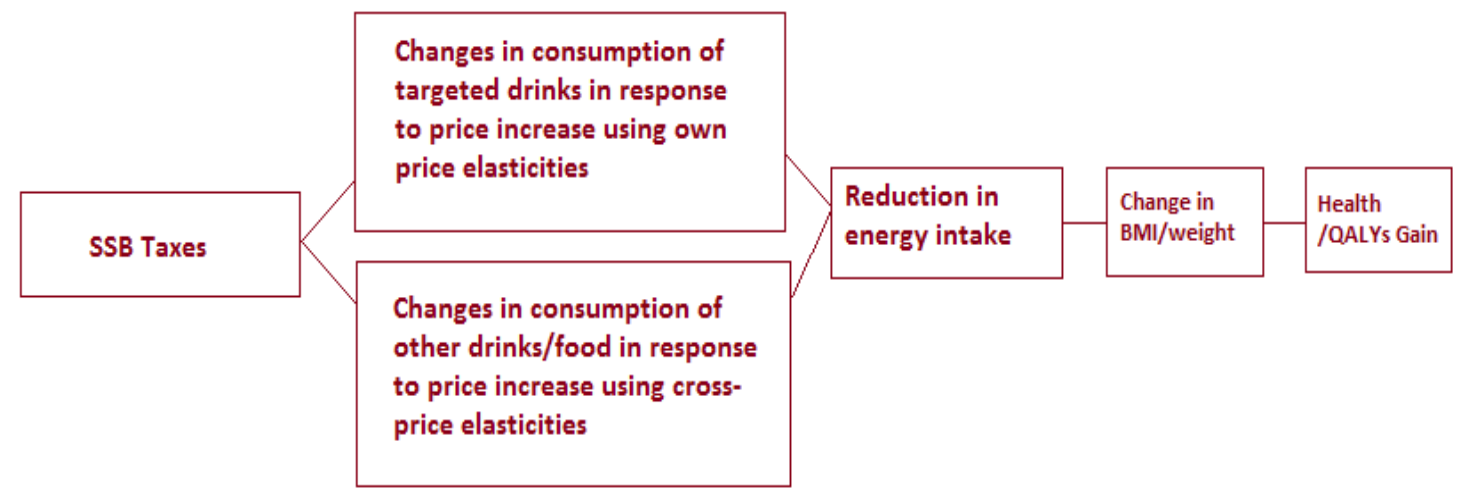

Figure 2.1: Channels of Benefits from SSB taxes

A specific disease model was often applied to predict the effects on specific disease outcomes in the selected studies. Changes in body mass index (BMI), reductions in disease burden and healthcare expenditures, DALYs averted, and QALYs gained were estimated using the Cardiovascular (CVD) Policy Model or the Australian Assessing 
Cost-Effectiveness (ACE) model (Lal et al., 2017; Cobiac et al.; Long et al., 2016; Mekonnen, 2013; Sánchez-Romero, 2016). These models are Markov cohort models which included different health states with assigned utility scores (e.g., QALYs or DALYs), where people in the model could transfer from one health state to the other with a transitional probability in each cycle. Costs were included in the models along with health utility values; outputs were the total QALYs or DALYs and costs over a given time. Given the QALYs/DALYs and costs, incremental QALYs and costs as well as the incremental cost-effectiveness ratio (ICER) could be calculated, expressed as cost per QALY gained. This approach is very conventional in health economic evaluation studies.

As Lal et al. (2017) pointed out in their limitations section, a common weakness of literature is that not all the societal costs are accounted for. The benefits of SSB taxes include tax revenue, change to consumption of SSBs and their substitutes, decreases in healthcare costs, improvements in health / disease risks, QALYs, and DALYs. The estimates of benefits may be biased as loss in productivity and other personal costs, such as time costs, travel costs, and friends' and family members' psychological loss due to the patient's sickness, are not accounted for. One potential response is to apply contingent valuation (CV), asking people's willingness-to-pay (WTP) for health improvement. The main possible advantage of WTP over QALYs and DALYs is that if the respondents have a comprehensive consideration of the loss from diseases, the societal burden and non-health personal costs will probably be better revealed by WTPs.

In addition, although health benefits (e.g., QALY gain or DALY reduction) were calculated as benefits of SSB taxes in many selected studies, nearly none of them further linked this to internalities. This problem matters as consumers do consider a proportion of harm of SSBs, rather than ignore all of it when they are purchasing and consuming SSBs. The only one which linked health change to internalities is Allcott, Lockwood, and Taubinsky's (2019) study which are described below.

\subsubsection{Internalities}

Allcott, Lockwood, and Taubinsky's (2019) measured the consumer bias using the 
"counterfactual normative consumer", and two types of internalities were included: imperfect information and self-control problems. Having investigated nutrition knowledge and excessive SSB consumption from 18,000 households, they found that if people had perfect self-control and were acknowledged as nutritionists or dietitians, SSB consumption per US household would decrease by 31 to 37 percent (Allcott, Lockwood, and Taubinsky, 2019).

Two concerns can be raised about their study. First, dieticians may value their health much higher than the average consumer. As a result, there is risk that the harm from internalities is overestimated in this study. Second, as Lal et al. pointed out, a common weakness is that not all the societal costs are accounted for (Lal et al., 2017). These costs include but are not limited to time costs, travel costs, and friends' and family members' psychological loss due to the patient's sickness, and loss in productivity and income due to illness. As a result, internalities may be underestimated. A potential response to this is to apply CV to ask general people's willingness-to-pay (WTP) for health risk reduction associated with excessive SSB consumption.

\subsubsection{Externalities}

Reductions in healthcare expenditure have been identified in many selected studies as benefits of SSB taxes, but few of them link this to externalities. The exception is Allcott, Lockwood, and Taubinsky. Instead of estimating externalities themselves, they calculated them from summarizing previous scholars' work. Allcott, Lockwood, and Taubinsky (2019) concluded that the externality of SSBs is approximately $\$ 0.85$ per ounce. Their conclusion was drawn according to the following two studies. Wang et al. (2012) estimated that one ounce of SSB consumption would increase healthcare expenditure by one cent on average. Given that 85 percent of the expenditure was covered by health insurance, while 15 percent of the medical expenditure was paid by patients, Allcott, Lockwood, and Taubinsky concluded that the externality of SSB which can be viewed as the costs to health system is approximately 0.85 cents per ounce (Allcott, Lockwood, and Taubinsky, 2019). However, given the heterogeneity in healthcare systems and costs cross countries, their findings are very unlikely to be applicable outside the US. Therefore, there is probably a need to measure externalities 
of SSBs in New Zealand.

\subsection{Other Concerns}

\subsubsection{Costs of SSB taxes}

Imposing a SSB tax can result in additional administration and transaction costs. Such a tax would be counterproductive if costs exceed benefits, and so there is a case for conducting a cost-benefit or cost-effectiveness analysis. Lal et al. (2017) estimated that legislation costs would be approximately AU\$1,090,000. Cobiac et al. (2017) calculated that the costs of implementation were AU\$22 million, and the threshold for an unhealthy food tax to be cost-effective was AU\$50,000 per DALY. Long et al. (2015) suggested that the costs of implementation were around US\$51 million in the first year; and that taxing SSBs was estimated to be cost saving.

Imposing a SSB tax might cause a loss in an individual's utility by depriving consumers' pleasure of drinking SSBs. This is seldom mentioned and discussed in public health literature and CUA/CEA studies. The exception is Lal et al. who supplement their CEA study with a simple discussion about deadweight loss (Lal et al., 2017). Another approach is to ask people their willingness-to-accept for the reduction of SSB consumption. The concern about deadweight loss also leads to a question: is taxing all beverages (including milk, tea, and coffee) better than taxing only SSBs? Admittedly, taxing all beverages may yield higher health benefits and tax revenue, while it may also cause a higher utility loss from depriving consumers from enjoying those drinks. If the marginal benefits of taxing all beverages are exceeded by the corresponding losses, then beverage taxes may be inferior to SSB taxes. This concern is quite important as it may have a crucial policy implication in reality. Given that this question has not been investigated by any of the selected studies, there may be a need to conduct a comparison to fill the gap in the future.

\subsubsection{Equity Concerns}

Another concern about SSB taxes is regressivity. As discussed previously, people with lower socioeconomic status are more likely to be intensive consumers, and the main tax burden will fall on them. Colchero et al. (2017) found that approximately 63 percent of the tax was collected from the lowest socioeconomic group in Mexico. As a 
result, this situation raises an equity concern.

On the other hand, people with lower socioeconomic status are more elastic to price, so their SSB consumption will decrease more significantly than their counterparts with higher socioeconomic status. This means that SSB taxes would have progressive health effects. In principle, the argument can be assessed by calculating indices which are often used to measure the extent to which benefits are concentrated in disadvantaged groups. Among the selected studies, one study adopted this approach. Lal et al. (2017) tested the equity/inequity in health gains associated with a SSB tax by calculating concentration indices. The concentration indices all took negative values, indicating that the health benefits were predominantly amongst the more disadvantaged groups, regardless of which tax base was used and how high the passthrough rates were (Lal et al., 2017). As a result, progressive health effects are seen in Lal et al. (2017).

Given the progressive health effects and regressive tax burden, the net benefits of SSBs are probably flat across the income distribution. Therefore, equity may not be a severe problem. However, there is still lack of evident to support this argument. A possible solution is to calculate the concentration indices of net welfare change (the difference between benefits and tax burden) in the future.

\subsubsection{Addiction}

Finally, some authors claim that sugar has addictive properties (Wilson and Hogan, 2017). Although this claim is controversial, it is possible that such an effect might change the optimal tax rate. If consumers are addicted to SSBs, they will be less responsive to price changes and their future consumption will depend on their consumption today; the more they drink today, the more they consume in the future (Wilson and Hogan, 2017). This problem may be addressed in a dynamic model in the future.

\subsection{Conclusions}

This literature review found that the socially optimal level of SSB taxes has been studied by Griffith et al., Marron, and Allcott, Lockwood, and Taubinsky. Griffith et al. and Marron derived the socially optimal tax formula from a utility maximization 
model. Their outcomes may be further generalized by including substitutes and compliments. Allcott, Lockwood, and Taubinsky derived the social-optimal tax formula with substitutes and compliments, but they still left room for future studies to investigate different tax bases at their socially optimal levels with considerations of substitutes and complements. In addition, other beverages such as fruit drink, milk drink, and energy drink probably should be included as substitutes in the model, but tobacco should be excluded.

There have been a few studies compared different tax bases, and nutrient taxes have been found to be more effective in decreasing energy intake. However, their findings probably should be questioned, because different tax bases were not compared at their socially optimal levels. In addition, a few studies compared the tax bases under the "economic equivalent taxes" assumption. This assumption is thought quite weak as there is no reason to keep the tax rates "economically equivalent" when tax base is changed. The best tax rate should be imposed, given the tax base the government has. Many studies suggested that SSB taxes could improve people's health, but other studies found such a tax also could be ineffective. Reported benefits of SSB taxes included tax revenue, reduction in health risks, and decreases in healthcare costs. Some studies estimated the costs of imposing a SSB tax; and a SSB could be beneficial to society as long as certain conditions hold. Although health benefits (e.g., QALY gain or DALY reduction) were calculated as benefits of SSB taxes in many selected studies, only Allcott, Lockwood, and Taubinsky further linked this to internalities. They estimated the internality from comparing dieticians' consumption with general people's consumption. However, given that dieticians may value their health higher than the average consumer, their estimated harm from internalities is probably overestimated.

The costs from externalities have been estimated by Allcott, Lockwood, and Taubinsky. Given the heterogeneity in healthcare systems and costs cross countries, their findings are very unlikely to be applicable outside the US. Therefore, there is probably a need to measure externalities of SSBs in New Zealand.

Finally, it may be meaningful to investigate whether taxing all beverages are superior 
to taxing SSBs in the future, and equity concerns may be addressed by calculating concentration indices for the net welfare gain from imposing taxes. 


\section{Utility-Maximization Model}

\subsection{Introduction}

In this chapter, we construct a theoretical model to answer the research questions listed in chapter 1. The model features multiple soft drinks, and accounts for complements and substitutes of those drinks. In addition, we also investigate the effects of SSB taxes on social welfare, and further determine which tax base is the most favorable.

As mentioned in the literature review section, no previous study has compared different tax bases at their socially optimal levels, using a utility-maximization model with consideration of substitutes. Creedy (2016) derived the effects of substitutes without consideration of social optimality. The socially optimal level of SSB taxes was studied by Allcott, Lockwood, and Taubinsky (2019), but they left room for future studies to investigate different tax bases at their socially optimal levels. In addition, we believe that the discussion should not follow Grummon et al.'s "economically equivalent taxes" assumption in which a tax rate is fixed for different tax bases (Grummon et al., 2019). This is because there is no reason to keep the tax rates "economically equivalent" when the tax base is changed. Instead, the best tax rate should be imposed, given the tax base the government has. Finally, we have identified that Grummon et al.'s model can be further extended by (1) comparing different tax bases (including ad valorem taxes) at their optimal levels, and (2) allowing for the effects of complements and substitutes.

Following the discussion above, we first set up a simple benchmark model of the optimal corrective tax. The model builds on Marron (2015) simple optimal tax model, but without distributional concerns. The familiar result is that the corrective tax should be set equal to the proportion of the marginal harm that the consumer does not 
account for. Then an extended model is introduced, which considers different commodities and allows for substitution and complementary effects. Finally, in order to identify the most and least favorable tax base, a quadratic functional form for utility is constructed to measure the welfare change associated with SSB taxes.

\subsection{A Simple Model}

Consider homogeneous consumers who purchase a single SSB. They have the following quasi-linear utility function:

$$
\begin{aligned}
U & =v(q)-p q-\beta H_{I} q-0 * H_{E} q \\
& =v(q)-p q-\beta H_{I} q
\end{aligned}
$$

where $\mathrm{p}$ and $\mathrm{q}$ denote the price of the SSB and consumption of the SSB in litres, respectively. The sub-utility function $\mathrm{v}(\mathrm{q})$ is the money metric utility from the SSB (the pleasure of consuming the SSB) which is assumed to be increasing in $\mathrm{q}$

( specifically, $\frac{d v(q)}{d q}>0$, ), concave $\left(\frac{d^{2} v(q)}{d q^{2}}<0\right)$, and continuously differentiable. $U(q)$ is the consumer's perception of 'net gain' from the SSB, that is, the difference between the pleasure of consuming the SSB and its cost, minus the proportion of health harm accounted for by the consumer.

$\mathrm{H}_{\mathrm{I}}$ is the marginal health-related personal harm and losses. It includes disutility from illness and personal medical expenditures, as well as other relevant personal costs (e.g., income loss due to illness). As with Marron (2015), because of lack of information about the increased health risks associated with SSB consumption and limited self-control due to a time-inconsistent preference for immediate pleasure, only a proportion of the health harm is acknowledged and considered by the consumers. Let $\beta \in(0,1)$ represent the proportion of the health harm considered by consumers, so that $(1-\beta) \mathrm{H}_{\mathrm{I}}$ is regarded as internalities, the harm the consumer does not account for. For example, if drinking a SSB generated ten dollars of internal costs per litre from increased health risks, and the individual already limits his or her consumption by taking into account the internal costs of 3 dollars, then $\beta=0.3$ and the internality is 7 dollars. If $\beta=1$, the consumer considers the full health harm, so their choice would not generate internalities. $\mathrm{H}_{\mathrm{E}}$ is the marginal cost of additional 
publicly funded medical expenditure associated with increased SSB consumption. Since the New Zealand public health system is largely funded by taxpayers, these costs can be viewed as fiscal externalities. In principle, there may be other externalities such as environmental costs. However, we believe these costs are relatively small, compared to health costs. Therefore, only fiscal externalities in terms of medical expenditure are considered in this study. In addition, we assume that consumers do not account for any externality costs in their consumption, and both $\mathrm{H}_{\mathrm{E}}$ and $\mathrm{H}_{\mathrm{I}}$ are constant, so that the marginal health harm $\mathrm{H}_{\mathrm{I}}$ equals the average health harm, and the marginal externality $\mathrm{H}_{\mathrm{E}}$ equals the average externality.

Next, as with Marron (2015), we assume that the soft drinks are provided by perfectly competitive firms at a per-unit cost of $\mathrm{c}$ (the beverage is produced in a constant marginal cost $\mathrm{c}$ and sold at the price which equals to the average/marginal cost $\mathrm{c}$ ), and $\tau$ is a volumetric tax imposed per unit. Thus, the price is determined by a zero-profit condition:

$$
\mathrm{p}=\mathrm{c}+\tau
$$

Substituting $\mathrm{p}$ into the private payoff yields:

$$
\begin{aligned}
U & =v(q)-(c+\tau) q-\beta H_{I} q \\
& =v(q)-c q-\tau q-\beta H_{I} q .
\end{aligned}
$$

This setup implicitly imposes $100 \%$ pass-through of the tax, as implied by perfect competition with a horizontal supply curve. The consumer's private first-order condition (FOC) of utility maximization is:

$$
\frac{d v(q)}{d q}-(c+\tau)-\beta H_{I}=0
$$

Now consider the problem of a benevolent government whose objective is to maximize social welfare. Unlike private consumers, the government is assumed to account for the full harm from the soft drink, $H_{E}$ plus $H_{I}$ (I assume that the planner knows $\mathrm{H}_{\mathrm{I}}$ ). In addition, I assume that tax revenues are returned to consumers as lump-sum transfers. The planner's objective is to maximize social welfare which can be defined as:

$$
\mathrm{W}=\mathrm{v}(\mathrm{q})-\mathrm{cq}-\left(\mathrm{H}_{\mathrm{E}}+\mathrm{H}_{\mathrm{I}}\right) \mathrm{q}
$$


Then the socially efficient consumption of soft drinks according to this value judgement is determined by the social FOC:

$$
\frac{\mathrm{dv}(\mathrm{q})}{\mathrm{dq}}-\mathrm{c}-\mathrm{H}_{\mathrm{E}}-\mathrm{H}_{\mathrm{I}}=0 .
$$

The two FOCs (3) and (5) are reconciled when the tax is set as follows:

$$
\tau=(1-\beta) \mathrm{H}_{\mathrm{I}}+\mathrm{H}_{\mathrm{E}} .
$$

Intuitively, the tax is the sum of the proportion of harm that would otherwise not be accounted for when making consumption decisions.

\subsection{An Extended Model \& Tax Impacts}

\subsubsection{An Extended Model}

Now we extend the preliminary model by assuming that there are $\mathrm{N}$ different types of drink, K of which are SSBs. Drinks can be complements, or substitutes of SSBs, or unrelated to them. As discussed above, the first-best is attainable in the simple setting with only one SSB, so it does not matter which tax base is chosen by the government. However, in this extended model with multiple SSBs and non-SSBs, a single tax instrument cannot implement the first-best. As a result, a second-best tax instrument is applied, and the choice of a tax base matters in this scenario.

Let the marginal cost of producing drink $\mathrm{j}$ be $\mathrm{c}_{\mathrm{j}}$. The harmful ingredient per litre of that drink is denoted by $\mathrm{r}_{\mathrm{j}}$. We assume that the total harm of consuming soft drinks $\left(\mathrm{H}_{\mathrm{I}}\right.$ and $\mathrm{H}_{\mathrm{E}}$ ) is proportional to the total amount of ingested harmful ingredients. Now the tax rate must reflect a compromise between the different levels of distortion associated with different drinks. Let $b_{j}$ denote the tax base for drink $j$. The tax base determines the marginal effect of tax on price. In this study, we consider three different possibilities for a tax base:

(i) litres of drink ( $b_{j}=1$, e.g., volumetric taxes),

(ii) (pre-tax) expenditure on drink $\mathrm{b}_{\mathrm{j}}=\mathrm{c}_{\mathrm{j}}$, i.e., ad valorem taxes or

(iii) the harmful ingredient contained in a litre of drink $\left(b_{j}=r_{j}\right)$.

Given that the tax base for drink $\mathrm{j}$ is $\mathrm{b}_{\mathrm{j}}$, the consumer's effective tax payment is: 


$$
\tau \sum_{j=1}^{K} q_{j} b_{j} .
$$

As with the simple model, the supply of each beverage is perfectly elastic and so pass-through of tax is $100 \%$. Therefore, the post-tax price of beverage $j$ is $p_{j}=c_{j}+\tau b_{j}$. In addition, as in the previous model, the consumer takes into account only a proportion $\beta$ of the health harm. Now the consumer's objective function is:

$$
U=v\left(q_{1}, \ldots, q_{n}\right)-\sum_{j=1}^{N} c_{j} q_{j}-\tau \sum_{j=1}^{K} q_{j} b_{j}-\beta H_{I} \sum_{j=1}^{N} q_{j} r_{j} .
$$

Compared with equation (2), expenditures on drinks (excluding tax payments) are $\sum_{j=1}^{N} c_{j} q_{j}$ rather than $c q$, because now there are $n$ beverages. Tax payments are now $\tau \sum_{j=1}^{K} q_{j} b_{j}$ rather than $\tau q$, because there are $k$ SSBs which are taxed. Finally, the harm considered by the consumer becomes $\beta H_{I} \sum_{j=1}^{N} q_{j} r_{j}$, because now there are $n$ beverages, and health harm per litre is $\mathrm{H}_{\mathrm{I}} \mathrm{r}_{\mathrm{j}}$. The sub-utility function $\mathrm{v}\left(\mathrm{q}_{1}, \ldots, \mathrm{q}_{\mathrm{n}}\right)$ is concave and increasing in all arguments. The private FOC with respect to consumption of a SSB $\mathrm{j}$ is:

$$
M V_{j}-c_{j}-\tau b_{j}-\beta H_{I} r_{j}=0,
$$

where $\mathrm{MV}_{\mathrm{j}}=\frac{\partial \mathrm{v}}{\partial \mathrm{q}_{\mathrm{j}}}$. The FOC with respect to a non-SSB is (for a non-SSB, the marginal impact of $\tau$ on $\mathrm{p}_{\mathrm{j}}$ is zero, because non-SSBs are not taxed):

$$
M V_{j}-c_{j}-0-\beta H_{I} r_{j}=0
$$

As with the simple model, the government is assumed to account for the full harm from the soft drink, $H_{E}$ plus $H_{I}$. Therefore, the social planner's objective is:

$$
v\left(q_{1}, \ldots, q_{n}\right)-\sum_{j=1}^{N} c_{j} q_{j}-\left(H_{I}+H_{E}\right) \sum_{j=1}^{N} q_{j} r_{j}
$$

Compared with equation (4), expenditures on drinks (exclude tax payments) are $\sum_{j=1}^{N} c_{j} q_{j}$ rather than $\mathrm{cq}$, because now there are $\mathrm{n}$ beverages. The total harm (health harm plus costs from externalities) is now $\left(H_{I}+H_{E}\right) \sum_{j=1}^{N} q_{j} r_{j}$, because there are $n$ beverages, and total harm per litre is $\left(\mathrm{H}_{\mathrm{E}}+\mathrm{H}_{\mathrm{I}}\right) \mathrm{r}_{\mathrm{j}}$. The social planner's FOC with respect to $\tau$, the tax rate, is: 


$$
\sum_{j=1}^{N}\left[M V_{j}-c_{j}-H_{E} r_{j}-H_{I} r_{j}\right] \frac{d q_{j}}{d \tau}=0
$$

where $\frac{d q_{j}}{d \tau}$ is the marginal impact of the tax on quantity consumed of a beverage $\mathrm{j}$.

Substituting (7) and (8) into (9), yields:

$$
\sum_{j=1}^{K}\left[\tau b_{j}-(1-\beta) H_{I} r_{j}-H_{E} r_{j}\right] \frac{d q_{j}}{d \tau}+\sum_{j=K+1}^{N}\left[0-(1-\beta) H_{I} r_{j}-H_{E} r_{j}\right] \frac{d q_{j}}{d \tau}=0 .
$$

Solving (10) for the tax rate yields:

$$
\tau=\left[(1-\beta) H_{I}+H_{E}\right] \frac{\sum_{j=1}^{N} r_{j} \frac{d q_{j}}{d \tau}}{\sum_{j=1}^{K} b_{j} \frac{d q_{j}}{d \tau}}
$$

The optimal corrective tax is now expressed as three terms: (i) $(1-\beta) H_{I}$ is the proportion of health harm that is not reflected in consumption, (ii) $\mathrm{H}_{\mathrm{E}}$ is the marginal harm of consumption associated with externalities, and (iii) $\frac{\sum_{j=1}^{N} r_{j} \frac{d q_{j}}{d \tau}}{\sum_{j=1}^{K} b_{j} \frac{d q_{j}}{d \tau}}$ is the scaler for the tax which reflects tax impacts.

When a volumetric tax is applied, $b_{j}=1$, so that $\frac{\sum_{j=1}^{N} r_{j} \frac{d q_{j}}{d \tau}}{\sum_{j=1}^{K} b_{j} \frac{d q_{j}}{d \tau}}=\frac{\sum_{j=1}^{N} r_{j} \frac{d q_{j}}{d \tau}}{\sum_{j=1}^{K} \frac{d q_{j}}{d \tau}}$. The scaler is the ratio of the harmful ingredient weighted sum of marginal tax impacts for all beverages to the weighted sum of marginal tax impacts for SSBs;

When an ad valorem tax is applied, $b_{j}=c_{j}$, so that $\frac{\sum_{j=1}^{N} r_{j} \frac{d q_{j}}{d \tau}}{\sum_{j=1}^{K} b_{j} \frac{d q_{j}}{d \tau}}=\frac{\sum_{j=1}^{N} r_{j} \frac{d q_{j}}{d \tau}}{\sum_{j=1}^{K} c_{j} \frac{d q_{j}}{d \tau}}$. The scaler is the ratio of the harmful ingredient weighted sum of marginal tax impacts for all beverages to the price weighted sum of marginal tax impacts for SSBs;

When a sugar tax is applied, $b_{j}=r_{j}$, so that $\frac{\sum_{j=1}^{N} r_{j} \frac{d q_{j}}{d \tau}}{\sum_{j=1}^{K} b_{j} \frac{d q_{j}}{d \tau}}=\frac{\sum_{j=1}^{N} r_{j} \frac{d q_{j}}{d \tau}}{\sum_{j=1}^{K} r_{j} \frac{d j}{d \tau}}$. The scaler is the ratio of the harmful ingredient weighted sum of marginal tax impacts for all beverages to the harmful ingredient weighted sum of marginal tax impacts for SSBs.

\subsubsection{Tax Impacts}

Next, the expressions for the marginal impacts of the tax on quantities consumed $\frac{\mathrm{dq}}{\mathrm{d} \tau}$, will be derived. As we assume that the supply of each beverage is perfectly elastic and so pass-through of tax is $100 \%$, then the post-tax price of beverage is $p=c+\tau b$. 
Consequently, the marginal impact of $\tau$ on $p$ is $b$ for SSBs, and zero for other drinks respectively. Now the marginal impact of $\tau$ on consumption of drink $\mathrm{j}$ is the sum of the effects through each of the K prices of SSBs:

$$
\frac{\partial \mathrm{q}_{\mathrm{j}}}{\partial \tau}=\sum_{\mathrm{i}=1}^{\mathrm{K}} \frac{\partial \mathrm{q}_{\mathrm{j}}}{\partial \mathrm{p}_{\mathrm{i}}} \frac{\mathrm{dp_{i }}}{\mathrm{d} \tau}=\sum_{\mathrm{i}=1}^{\mathrm{K}} \frac{\partial \mathrm{q}_{\mathrm{j}}}{\partial \mathrm{p}_{\mathrm{i}}} \mathrm{b}_{\mathrm{i}}
$$

In addition, it is going to be convenient if these expressions are phrased in terms of elasticities, as this is what usually gets reported in empirical studies. Given the definition of a cross-price elasticity:

$$
\frac{\partial \mathrm{q}_{\mathrm{j}}}{\partial \mathrm{p}_{\mathrm{i}}}=\varepsilon_{\mathrm{ij}} \frac{\mathrm{q}_{\mathrm{j}}}{\mathrm{p}_{\mathrm{i}}}
$$

we can derive an expression for $\frac{d q_{j}}{d \tau}$ in terms of $\varepsilon_{i j}$.

Substitute (13) into (12):

$$
\frac{d q_{j}}{d \tau}=\sum_{i=1}^{K} \frac{q_{j}}{p_{i}} \varepsilon_{i j} b_{i} .
$$

Finally, substitute (14) into the expression for the scaling factor (11):

$$
\frac{\sum_{j=1}^{N} r_{j} \frac{d q_{j}}{d \tau}}{\sum_{j=1}^{K} b_{j} \frac{d q_{j}}{d \tau}}=\frac{\sum_{j=1}^{N}\left(r_{j} \sum_{i=1}^{K} \varepsilon_{i j} \frac{q_{j}}{p_{i}} b_{i}\right)}{\sum_{j=1}^{K}\left(b_{j} \sum_{i=1}^{K} \varepsilon_{i j} \frac{q_{j}}{p_{i}} b_{i}\right)} .
$$

Therefore, the socially optimal levels are:

$$
\begin{gathered}
\tau=\left[(1-\beta) H_{I}+H_{E}\right] \frac{\sum_{j=1}^{N} r_{j} \frac{d q_{j}}{d \tau}}{\sum_{j=1}^{K} b_{j} \frac{d q_{j}}{d \tau}} \\
=\left[(1-\beta) H_{I}+H_{E}\right] \frac{\sum_{j=1}^{N} r_{j} \sum_{i=1}^{K} \varepsilon_{i j} \frac{q_{j}}{p_{i}} b_{i}}{\sum_{j=1}^{K} b_{j} \sum_{i=1}^{K} \varepsilon_{i j} \frac{q_{j}}{p_{i}} b_{i}},
\end{gathered}
$$

Specifically:

(i) for volumetric taxes, $b_{j}=1$,

$$
\frac{\sum_{j=1}^{N} r_{j} \frac{d q_{j}}{d \tau}}{\sum_{j=1}^{K} b_{j} \frac{d j}{d \tau}}=\frac{\sum_{j=1}^{N} r_{j} \frac{d q_{j}}{d \tau}}{\sum_{j=1}^{K} \frac{d q_{j}}{d \tau}}=\frac{\sum_{j=1}^{N} r_{j} \sum_{i=1}^{K} \varepsilon_{i j} \frac{q_{j}}{p_{i}}}{\sum_{j=1}^{K} \sum_{i=1}^{K} \varepsilon_{i j} \frac{q_{j}}{p_{i}}},
$$

(ii) for an ad valorem tax $b_{j}=c_{j}$,

$$
\frac{\sum_{j=1}^{N} r_{j} \frac{d q_{j}}{d \tau}}{\sum_{j=1}^{K} b_{j} \frac{d q_{j}}{d \tau}}=\frac{\sum_{j=1}^{N} r_{j} \frac{d q_{j}}{d \tau}}{\sum_{j=1}^{K} c_{j} \frac{d q_{j}}{d \tau}}=\frac{\sum_{j=1}^{N} r_{j} \sum_{i=1}^{K} \varepsilon_{i j} \frac{q_{j}}{p_{i}} c_{i}}{\sum_{j=1}^{K} c_{j} \sum_{i=1}^{K} \varepsilon_{i j} \frac{q_{j}}{p_{i}} c_{i}},
$$

(iii) for a sugar tax, $b_{j}=r_{j}$, 


$$
\frac{\sum_{j=1}^{N} r_{j} \frac{d q_{j}}{d \tau}}{\sum_{j=1}^{K} b_{j} \frac{d q_{j}}{d \tau}}=\frac{\sum_{j=1}^{N} r_{j} \frac{d q_{j}}{d \tau}}{\sum_{j=1}^{K} r_{j} \frac{d q_{j}}{d \tau}}=\frac{\sum_{j=1}^{N} r_{j} \sum_{i=1}^{K} \varepsilon_{i j} \frac{q_{j}}{p_{i}} r_{i}}{\sum_{j=1}^{K} r_{j} \sum_{i=1}^{K} \varepsilon_{i j} \frac{q_{j}}{p_{i}} r_{i}} .
$$

\subsubsection{The Socially Optimal Levels for Sugary Drink Taxes}

As discussed previously, SSB taxes may not be effective, as consumers may switch to other sugary foods which are plausibly substitutes for SSBs. Given this possibility, it may be meaningful to investigate whether taxing all sugary beverages is superior to taxing only SSB. The socially optimal levels for beverage taxes and the welfare associated with such taxes can be derived using the same method. Now we assume that there are $\mathrm{N}$ different types of beverages, and all of them are taxed. The new formula for the optimal tax rate is the same as the previous one, except that $\mathrm{K}$ now has been replaced by $\mathrm{N}$. Therefore, the socially optimal levels for beverage taxes are:

$$
\tau=\left[(1-\beta) H_{I}+H_{E}\right] \frac{\sum_{j=1}^{N} r_{j} \frac{d q_{j}}{d \tau}}{\sum_{j=1}^{N} b_{j} \frac{d q_{j}}{d \tau}} .
$$

and the expression for the scaling factor is:

$$
\frac{\sum_{j=1}^{N} r_{j} \frac{d q_{j}}{d \tau}}{\sum_{j=1}^{N} b_{j} \frac{d q_{j}}{d \tau}}=\frac{\sum_{j=1}^{N} r_{j} \sum_{i=1}^{N} \varepsilon_{i j} \frac{q_{j}}{p_{i}} b_{i}}{\sum_{j=1}^{N} b_{j} \sum_{i=1}^{N} \varepsilon_{i j} \frac{q_{j}}{p_{i}} b_{i}} .
$$

\subsection{Constructing a Welfare Measurement}

In this section, a welfare measurement is developed to evaluate the welfare change due to SSB taxes. This measurement can be used to investigate which tax base is the best in a certain environment. While the previous result is informative about the optimal corrective tax rate, it does not tell us the magnitude of the welfare change due to such a tax. Consequently, it does not reveal which tax base is the most beneficial. In order to provide a specific welfare measurement, we need to make an assumption about the functional form of the utility function $U\left(q_{1}, \ldots, q_{n}\right)$. Different studies and their estimate of elasticities implicitly assume different utility functions (Griffith, O’Connell, and Smith, 2017). As with O' Donoghue and Rabin (2006) and Griffith and O'Connell (2018), we assume the simplest form which is quadratic (a quasi-linear) utility. This assumption guarantees that the SSB consumption and welfare change are independent of income, and the marginal utility of income is constant (Griffith, O'Connell, and Smith, 2017). In addition, the approach is equivalent to using a 
multi-commodity deadweight loss measure, with linear approximations of demand curves (Jacobsen et al., 2016; Snyder \& Nicholson, 2015).

Now, we are going to derive the demand curve and the quadratic utility function. Based on the definition of elasticity, we can infer the slope of the demand curves from estimated own and cross-price elasticities:

$$
\frac{\partial q_{i}}{\partial p_{j}}=\varepsilon_{i j} \frac{q_{i}}{p_{j}}=B_{i j}
$$

Let matrix B be an array of these slopes of demand curves. Consumer theory requires the matrix of slopes of the demand curves to be symmetric and negative semi-definite, but empirical estimates usually do not exactly meet these requirements. One reason is that income effects may be important, but we think this is unlikely for soft drinks, because SSB consumption only accounts for a very small proportion of the whole expenditure (Ni Mhurchu et al., 2013). Hence, a SSB tax is unlikely to generate a heavy substantial tax burden and cause a substantial income loss to the average consumer. Consequently, a more plausible reason is sampling error and imprecision of empirical estimates.

Sharma et al. set elasticities equal to zero if those estimates were not statistically significant. In this study, we follow their approach. In addition, we impose symmetry by taking an average of the slope matrix and its transpose.

In matrix notation, if $\mathrm{B}$ is the matrix of estimated slopes, then after imposing symmetry, the demand functions are now:

$$
\mathrm{q}=\mathrm{a}+0.5 *\left[\mathrm{~B}+\mathrm{B}^{\mathrm{T}}\right] \mathrm{p},
$$

where $\mathrm{q}$, a, and $\mathrm{p}$ are vectors (one element for each good).

Let the initial (observed) prices and quantities be the vectors $\mathrm{p}_{0}$ and $\mathrm{q}_{0}$, so that $\mathrm{q}_{0}=\mathrm{a}-$ $\mathrm{Bp}_{0}$. Then we can back out the intercepts of the demand curves as:

$$
\mathrm{a}=\mathrm{q}_{0}-0.5 *\left[\mathrm{~B}+\mathrm{B}^{\mathrm{T}}\right] \mathrm{p}_{0} .
$$

For welfare analysis, it is going to be convenient to work with inverse demand curves:

$$
\mathrm{p}=\left\{0.5 *\left[\mathrm{~B}+\mathrm{B}^{\mathrm{T}}\right]\right\}^{-1} \mathrm{q}-\left\{0.5 *\left[\mathrm{~B}+\mathrm{B}^{\mathrm{T}}\right]\right\}^{-1} \mathrm{a}
$$

That is, $-\left\{0.5 *\left[\mathrm{~B}+\mathrm{B}^{\mathrm{T}}\right]\right\}^{-1} \mathrm{a}$ is a vector that gives us the intercepts of the inverse demand curves, and $\left\{0.5 *\left[\mathrm{~B}+\mathrm{B}^{\mathrm{T}}\right]\right\}^{-1}$ is a matrix that gives us the slopes of these 
inverse demand curves.

If consumers maximize private utility and the inverse demand curves are linear, $p=$ $\mathrm{m}-\mathrm{nq}$, then this implies:

$$
\mathrm{U}(\mathrm{q})=\mathrm{m}^{\mathrm{T}} \mathrm{q}-0.5 \mathrm{q}^{\mathrm{T}} \mathrm{nq}-\mathrm{pq},
$$

where $\mathrm{m}=-\left\{0.5 *\left[\mathrm{~B}+\mathrm{B}^{\mathrm{T}}\right]\right\}^{-1} \mathrm{a}$ and $\mathrm{n}=-\left\{0.5 *\left[\mathrm{~B}+\mathrm{B}^{\mathrm{T}}\right]\right\}^{-1}$.

Then welfare is:

$$
\begin{gathered}
W=U(q)-r^{T} q\left(H_{I}+H_{E}\right) \\
=m^{T} q-0.5 q^{T} n q-p^{T} q-r^{T} q\left(H_{I}+H_{E}\right) .
\end{gathered}
$$

The welfare change due to SSB taxes is:

$$
\Delta \mathrm{W}=\mathrm{U}\left(\mathrm{q}_{1}\right)-\mathrm{U}\left(\mathrm{q}_{0}\right)+\tau \mathrm{q}_{1}+\left(\mathrm{H}_{\mathrm{I}}+\mathrm{H}_{\mathrm{E}}\right) \mathrm{r}^{\mathrm{T}}\left(\mathrm{q}_{0}-\mathrm{q}_{1}\right),
$$

Here $\mathrm{p}_{0}$ and $\mathrm{q}_{0}$ are the initial (observed) prices and quantities; $\mathrm{p}_{1}$ and $\mathrm{q}_{1}$ are the post-tax prices and quantities. The decrease in utility due to reduction in beverage consumption is $\mathrm{U}\left(\mathrm{q}_{1}\right)-\mathrm{U}\left(\mathrm{q}_{0}\right) ; \tau \mathrm{q}_{1}$ is tax revenue, and so deadweight loss due to taxes is $\mathrm{U}\left(\mathrm{q}_{1}\right)-\mathrm{U}\left(\mathrm{q}_{0}\right)+\tau \mathrm{q}_{1}$; and harm reduction due to taxes is $\left(\mathrm{H}_{\mathrm{I}}+\right.$ $\left.\mathrm{H}_{\mathrm{E}}\right) \mathrm{r}^{\mathrm{T}}\left(\mathrm{q}_{0}-\mathrm{q}_{1}\right)$.

\subsection{Discussion \& Conclusion}

The model has the following limitations. First, we assumed that the total harm of consuming soft drinks is proportional to the total amount of calories in this study. However, the total harm of consuming soft drinks also can be related to the total amount of carbon dioxide and aspartame or stevia each beverage contains. Studies have shown that carbon dioxide could reduce calcium absorption and thereby, damaging bone health, causing dental problems and other related diseases (Kim et al., 2017; Malik, 2017). Another similar concern is that some zero-calorie SSBs may contain aspartame or stevia which may be harmful to health. In particular, the association between aspartame and migraine headaches has been identified in numbers of studies (Tandel, 2011; Narain, Kwok and Mamas, 2016). Given this, future studies should take these factors into consideration, accounting for all the potential harmful ingredients.

Second, some authors claim that sugar has addictive properties (Wilson and Hogan, 2017). Although this claim is controversial, it is possible that such an effect might 
change the optimal tax rate. If consumers are addicted to SSBs, they will be less responsive to price changes, and their future consumption will depend on their consumption today. The more they drink today, the more they will consume in the future (Wilson and Hogan, 2017). In addition, if SSBs are addictive, there will be substantial differences between short-term elasticities (when there is a one-off price change) and long-term elasticities (when there is a permanent price change). In order to address this problem, a dynamic model may be constructed in the future.

Third, income effects of SSB taxes are ruled out by the assumption of a quasi-linear utility function. This concern is somewhat moderated by the argument that income effects are unlikely to be important for SSBs, because SSB consumption only accounts for a very small proportion of total food expenditure in New Zealand (Ni Mhurchu et al., 2013). Furthermore, if utility is linear in money, consumers will be risk neutral in regards to the possibilitiy of income loss. This contradicts the fact that in real life people often purchase insurance against loss of income. Therefore, it would still be meaningful to drop the quasi-linear utility assumption and include the income effect in a future extension of this study.

Fourth, the model assumes a representative agent. In reality, there can be substantial differences between consumers. For example, there may be heterogeneities in the marginal harm parameters and the degree self-control. In order to address this problem, a sensitivity analysis should be conducted in the simulation (e.g. different values of $\beta$ ). In future work, I also plan to investigate a model with a distribution of different types of consumers.

Another potential weakness is that SSBs are assumed to be provided by perfectly competitive firms so that the entire tax will be passed to consumers. This assumption is somewhat contradicted by the fact that the beverage industry is dominated by large firms with market power (e.g., Coca Cola and Pepsi) and therefore, prices of SSB probably far exceed their marginal costs. Consequently, as discussed in the literature review in the last chapter, the pass-through rates vary from around $50 \%$ to $100 \%$ in the real world (Falbe et al.; 2015; Colantuoni, 2014). In addition, market power may have a substantial effect on the optimal tax rate. As shown by Calcott and Petkov 
(2013) optimal taxes on cigarettes are lower in an oligopolistic market. Therefore, there is a need for future studies to consider a model of optimal taxation of SSBs in which they are supplied by oligopolistic firms.

As discussed in the first chapter, the association between SSB consumption and obesity and diabetes has been identified in many studies (Hu, 2013). However, whether taxes alone can reduce SSB consumption and further generate health benefits is controversial. In the model, we implicitly assumed that an increase in prices can result in a decrease in consumption and energy intake, and the decrease in energy intake can further lower risk factors (e.g., obesity) and finally yield health benefits. But in reality, a drawback in any of these steps can make SSB taxes fail to reach their policy goals.

Finally, equity concerns about SSB taxes, and the distribution of tax revenue, are not addressed in this study. Since people with lower socioeconomic status are more likely to be intensive consumers and hence suffer the heaviest tax burden, SSB taxes may raise equity concerns. One possible solution to address the equity concern is an income transfer. This has been modelled by Allcott, Lockwood, and Taubinsky (2019) who have shown that $\sin$ tax regressivity can be offset by income tax reforms, if differences in consumption are due to income effects, rather than preference heterogeneity. Another solution could be subsidies for healthy foods, especially those low energy and nutrient beverages, for instance, low-fat milk. Such subsidies might be useful because (1) the income effects of SSB taxes may decrease healthy food consumption (although we think they should be quite small) and (2) given the substation relationship between sugary drinks and those healthy foods, such subsidies may further reduce SSB consumption as a result of decrease in healthy foods price. Therefore, there is probably a need for future studies to investigate the socially optimal levels of health food subsidies which are used as a targeted transfer of tax revenues.

The contribution of our model includes following. Compared with Allcott, Lockwood, and Taubinsky (2019), our model compares different tax bases at its socially optimal levels and therefore moves forward a step. In addition, unlike their study in which 
cigarettes are treated as substitutes of SSBs, we only modeled the substitution between beverages as we believe it is more likely for a consumer to switch from a SSB to another beverage (such as milk, fruit juice, and coffee), rather than cigarettes. A related model is discussed in Grummon et al.'s study. Compared with their model, our approach is probably more advantaged because it compares different tax bases at its socially optimal levels, without the "economically equivalent" assumption. Since government is assumed to choose a tax rate to maximize social welfare given a tax rate, it is pointless to assume the same tax rates. From this point of view, our model moves forward a step again. Moreover, ad valorem tax is compared with other tax bases in our model. We believe this is important as ad valorem taxes are probably the most commonly used tax base for SSB taxes and hence should not be neglected.

Finally, our model distinguishes SSBs from other drinks, which enables us a chance to compares SSB taxes with beverage taxes. Admittedly, taxing all beverages may yield higher health benefits and tax revenue, while it may also cause a higher utility loss from depriving consumers from enjoying those drinks. If benefits are dominated by loss, then beverage taxes may be inferior to SSB taxes. This concern can be investigated using our model and calibrated with real world data, the results of which may have an important policy implication in reality.

\subsection{What is Next?}

Since the marginal harm parameters $\mathrm{H}_{\mathrm{I}}$ and $\mathrm{H}_{\mathrm{E}}$ are included as components of the optimal tax formula and welfare measurement, their values must be estimated. As discussed in the literature review, although their values have been investigated by previous studies, these results are probably biased and inapplicable to New Zealand. Therefore, in the next two chapters, we are going to estimate the values of the marginal harm parameters in a New Zealand context. Chapter four discusses a contingent valuation (CV) study, the result of which is used to measure the proportion of health harm that is not reflected in consumption. In chapter five, a cost analysis is conducted to estimate the marginal harm of consumption associated with externalities. 


\section{Measuring the Harm of SSBs associated with Internalities}

\subsection{Introduction}

As discussed previously, to answer the research questions 1 to 4 , a theoretical model of the optimal corrective tax was constructed in chapter 3. Recall that the optimal-tax formula is:

$$
\tau=\left[(1-\beta) H_{I}+H_{E}\right] \frac{\sum_{j=1}^{N} r_{j} \frac{d q_{j}}{d \tau}}{\sum_{j=1}^{K} b_{j} \frac{d q_{j}}{d \tau}}
$$

where $H_{I}$ is the marginal health harm of SSB consumption, $\beta \in(0,1)$ represents the proportion of the harm considered by consumers, so that $(1-\beta) H_{I}$ is regarded as internalities, $H_{E}$ is the marginal externality cost from SSB consumption, and $\frac{\sum_{j=1}^{N} r_{j} \frac{d q_{j}}{d \tau}}{\sum_{j=1}^{K} b_{j} \frac{d q_{j}}{d \tau}}$ is the multiplier.

Since the marginal health harm $\mathrm{H}_{\mathrm{I}}$ is included as a component of the optimal tax formula, its value has to be estimated, if we want to calculate the socially optimal tax rate. As discussed in the literature review section, the monetary value of health harm from SSB consumption has been investigated by Allcott, Lockwood, and Taubinsky (2019), who compare dieticians' consumption with public consumption. However, their estimate may be biased, given that people who are more concerned with health are probably more likely to become dieticians. This leads to a risk that the harm from SSBs is overestimated. Therefore, it would be meaningful to estimate the monetary value of health harm using a different approach.

Following the discussion above, the objective of this chapter is to measure the monetary value of health harm from SSBs. The next section explains the method used to estimate the value of health harm.

\subsection{Methodology}


If we assume the marginal health harm of SSB consumption is constant, then it will be equal to the average health harm which can be calculated as the total harm divided by the total SSB consumption in a certain period. Let $\mathrm{H}_{\mathrm{t}}, \mathrm{N}_{\mathrm{ssb}}$, and $\mathrm{H}_{\mathrm{I}}$ denote total health harm, total SSB consumption, and the average health harm (equal to the marginal harm) respectively. The relationship between the three terms is:

$$
\mathrm{H}_{\mathrm{I}}=\frac{\mathrm{H}_{\mathrm{t}}}{\mathrm{Nssb}} \text {. }
$$

As discussed in the first chapter, the association between SSB consumption and various diseases has been identified in several studies. The more SSBs people consume, the higher is the disease risk they will develop. In addition, it should be noted that once people become sick, they would probably suffer from not only a decrease in health, but also non-health impacts such as loss in income. Consequently, the total health harm from SSB consumption can be calculated as the loss from diseases (decrease in health and other non-health losses) multiplied by the increased health risks from SSB consumption. Let $\mathrm{L}$ denote the loss from diseases, and $\mathrm{R}$ is the increased risk associated with SSB consumption, formula (4.1) now becomes:

$$
\mathrm{H}_{\mathrm{I}}=\frac{\mathrm{H}_{\mathrm{t}}}{\mathrm{Nssb}}=\frac{\mathrm{L} * \mathrm{R}}{\mathrm{Nssb}} \text {. }
$$

In order to investigate the value of $\mathrm{H}_{\mathrm{I}}, \mathrm{L}, \mathrm{R}$, and $\mathrm{N}_{\mathrm{ssb}}$ have to be estimated. The next few sections describe the methods used to measure the three terms.

\subsubsection{Increased Health Risks}

In order to measure the value of $\mathrm{R}$, a short literature review is conducted to investigate the potential diseases caused by excessive SSB consumption, as well as to determine the association between the increased health risks and SSB consumption. Literature was searched and further summarized systematically. Details are discussed below.

\subsubsection{Objectives}

The following questions will be addressed in this literature review:

1. How have previous studies classified drinkers as light-to-medium or heavy drinkers?

2. What are the main health outcomes of light-to-medium and heavy consumption of SSBs and ASBs? 
3. Which combination of health outcomes from question 2 above can be used as potential indicators of SSB and ASB harm?

4. How high are the risks of developing the health outcomes from question 2 among light-to-medium and heavy SSB and ASB drinkers, compared with nondrinkers?

\subsubsection{Search Strategy}

Studies published up to July $5^{\text {th }}$, 2017, were searched in databases including Medline (from 1994), EconLit (from 1800), Scopus (from 1960), and Web of Science - all data bases (from 1864), as well as the Cochrane Database of Systematic Reviews (from 1999). The key words used in the search were 'sweetened soda beverages', 'artificial sweetened beverages', 'health outcomes', 'harms' as well as 'risks'; the language was English. This effort initially resulted in more than 1,500 studies (including duplicates).

\subsubsection{Results of the Literature Review}

(a) Light-to-Medium Drinkers \& Heavy Drinkers

A scan of the titles of these studies showed that intensive drinkers were defined as people who consume at least one bottle or 1 serving of SSBs per day in most of the studies. People who consumed between once per month to six times per week were classified as light-to-medium drinkers; people who consumed less than once per month were identified as non-drinkers.

Based on these classifications and the observation that the majority of SSB cans and small bottles in supermarkets in Wellington, New Zealand vary from $200 \mathrm{ml}$ to $600 \mathrm{ml}$, we assume that the consumption per occasion is the average amount of the size of the cans - 400ml. Consequently, people who consume above $400 \mathrm{ml}$ per day on average (equivalent to 12 litres per month or 1,440 litres in ten years) are classified as intensive drinkers in this study. People who consumed between 0 and $400 \mathrm{ml}$ per day on average are classified as light-to-medium drinkers. Finally, people who do not drink SSBs are classified as non-drinkers.

(b) Potential Indicators of Harm from SSBs \& ASBs

The titles of these studies suggest that the majority focused on obesity, type- 2 diabetes, hypertension, stroke, and heart disease. A few other studies concentrated on cancer and dental problems. We decided to focus on diabetes, stroke, and heart diseases. The 
reasons why these three were selected are outlined below.

First, obesity and diabetes should not be combined. Some studies revealed that the risk ratio (RR) of diabetes between heavy drinkers and non-drinkers was not statistically significant after adjustment for body mass index (BMI). This suggests that BMI might account for some, or even all the diabetes excess risk (Schulz et al., 2004). Based on this evidence, if obesity and diabetes were combined, some of the risks would be double counted. Similarly, stroke should not be combined with hypertension, as hypertension has been found to be one of the most prevalent, independent, and modifiable risk factors for stroke in many studies (Bernstein et al., 2012).

Second, obesity and hypertension are typically regarded as health risk factors, while diabetes, stroke, and heart disease are more often treated as serious diseases which usually require urgent medical interventions and can cause several severe and even fatal complications if they are not controlled in time (Kyle, 2012). From this perspective, diabetes, stroke, and heart disease probably should be included, and obesity and hypertension should be excluded.

Third, though there were a few studies concentrating on cancer and dental problems, the association between health risks and SSB consumption was not clearly demonstrated. Besides, there were not many of these studies compared with those that concentrated on diabetes, stroke, and heart disease. Therefore, the studies focusing on cancer and dental problem were not included in this study.

(c) Risks of Diabetes, Stroke, \& Heart Disease associated with SSBs

The studies found in the initial search were screened again, based on the following criteria: (1) the study empirically compared the risk of type-2 diabetes, or stroke, or heart diseases between heavy drinkers or light-to medium drinkers and nondrinkers; (2) the risks were revealed by relative risk (RR), hazard ratio (HR), or risk difference; (3) risks were reported for the whole population rather than only for sub groups (for instance, young adults or minorities); (4) statistical significance was found for the difference between the groups at 5\% level; and (5) the duration of the trial was at least eight years.

The selection resulted in eleven randomized controlled trials (RCT) and two 
meta-analyses. These studies suggest that compared with non-drinkers, light-to-medium drinkers have 7\% - 9\% higher risk of developing diabetes, $2 \%-4 \%$ higher risk of developing heart disease, and 3\% - 12\% higher risk of developing stroke; heavy drinkers have 18\% - 83\% higher risk of developing diabetes, $19 \%-35 \%$ higher risk of developing heart disease, and 16\% - 83\% higher risk of developing stroke. Among the selected studies, two meta-analyses were found in which clinical evidence was well summarized, critically evaluated, and further analyzed with extensive details. In particular, Malik concluded that compared with non-drinkers, heavy drinkers had 26\% higher risk of diabetes (Malik, 2017). Narain's study found that compared with non-drinkers, heavy drinkers had $13 \%$ to $14 \%$ higher risk of stroke, and 22\% higher risk of heart disease (Narain, Kwok and Mamas, 2016). The information mentioned above was later used in developing the CV survey and further estimate the marginal harm of SSBs in this study. The details of the selected studies are summarized in Table 4.1 in Appendix.

Based on the findings of the literature review, compared with a non-drinker, the increased risk of diabetes, stroke, and heart disease of a light-to-medium drinker are $7 \%, 7 \%$, and $2 \%$ respectively in the base case in this study.

\subsubsection{SSB Consumption}

SSB consumption per person per year was estimated based on the following information.

Statistics indicate that the weekly household expenditure on soft drinks was approximately \$7.91 in 2018 (Food price index: November, 2018; available from Statistics NZ). Also, Statistics shows that the average price of SSBs was approximately $\$ 2.50$ per 1.5 litres in $2018 .^{3}$ Given this, the unit price of a SSB is approximately $\$ 2.5 / 1.5=\$ 1.667$ per litre. Therefore, the weekly household SSB consumption would be $\$ 7.91 / \$ 1.667=4.75$ litres.

The weighted average number of people per household was estimated to be approximately 3.50 (Baker et al., 2012). In this estimation, we assumed that for household size $=8$ or $>8$, the average number of people per household was assumed

${ }^{3}$ https://figure.nz/chart/WNZOpEoBKRyz4hBh-7p7mC5KFHBt4Rx4u 
to be 8 in the base case. The assumption should be a reasonable approximation as households with at least 8 persons constitute only $3 \%$ of all households in New Zealand. Given this, the uncertainty about household size was considered unlikely to have a substantial effect on results.

Based on the preceding information above, SSB consumption per person per week was $4.75 / 3.50=1.356$ litres, and SSB consumptions per person per year was $1.356 / 7 * 365=70.71$ litres.

\subsubsection{Losses from Diseases}

\subsubsection{Contingent Valuation}

This section describes the method used to measure the monetary value of losses from disease. Ideally, the measurement would include not only decreases in health, but also non-health losses such as decline in income. One possible method is to apply contingent valuation $(\mathrm{CV})$.

$\mathrm{CV}$ is an approach which is often used to measure the monetary value of non-market goods in environmental and natural resource economics (Goodstein, 2011). In recent years, this method has gradually been applied more frequently in health economics (Smith \& Sach, 2010). Questions in CV surveys are usually framed as 'how much are you willing to pay for $\mathrm{X}$ ?', or 'are you willing to pay $\$ Z$ for $X$ ?'. The reason why it is called $\mathrm{CV}$ is that the answers elicited from participants are contingent upon the questions asked (Smith \& Sach, 2010).

Compared with cost-utility analysis (CUA) in which health outcomes are usually assessed by quality-adjusted life years (QALYs) or disability-adjusted life years (DALYs), CV and cost-benefit analysis (CBA) may have some advantages. The main ones are monetization and aggregation of varied benefits into a single measure. Especially, compared with CUA, outcomes valued in CV or CBA research usually include not only health consequences, but also non-health outcomes, such as income loss and time costs (Smith and Sach, 2009).

Although CV has some advantages over CUA or cost-utility analysis (CEA), we did not find any study that has applied the CV method to harm caused by sweetened and sugary beverages (SSBs) and artificially sweetened beverages (ASBs). This may be 
because some people believe life and health are priceless. This opinion leads to an ethical concern that comparing health and life across individuals by assigning monetary value to these may be inappropriate (Liljas and Blumenschein, 2000). In addition, the reliability of $\mathrm{CV}$ studies is often threatened by hypothetical bias (Goodstein, 2011). Because questions in the CV survey are usually posed as mere possibilities, people are very likely to provide equally hypothetical and meaningless answers. Finally, embedding bias, strategic bias, and cultural bias, all may affect the results generated from the CV method (Goodstein, 2011).

While placing monetary value on aspects of life and health may be somewhat ethically unacceptable, and the results of CV survey may be affected by some biases, it is still worthwhile to assess the monetary value of harm from SSBs by applying the CV method, given its potential advantages.

Ideally, the losses from disease would be revealed by people's willingness-to-pay (WTP) for the reduction in health risk. Let WTP denote people's WTP for a $1 \%$ reduction in health risks. Now formula 4.2 becomes:

$$
\mathrm{H}_{\mathrm{I}}=\frac{\mathrm{L} * \mathrm{R}}{\mathrm{Nssb}}=\frac{\mathrm{WTP} * \mathrm{R}}{\mathrm{Nssb}} \text {. }
$$

Now the problem is to measure people's WTP for $1 \%$ health risk reduction. An easy way is to ask people directly how much they are willing to pay for a $1 \%$ health risk reduction. However, questions framed in the open-ended format have been shown to be very hard for people to answer in a few studies, and people's answers are more likely to be affected by serious hypothetical bias. In our pilot survey, this problem was also seen (details will be discussed in the pilot survey section). As a result, the open-ended format was abandoned. We switched to a stated preference survey. Details are discussed below.

\subsubsection{Survey}

\section{(a) Objective of the CV Survey}

The objectives of this contingent valuation survey were to measure people's WTP for diabetes, stroke, and heart disease risk reductions, and further to estimate the marginal harm from SSB consumption. 


\section{(b) Methodology of the CV Survey}

The methodology followed the choice experiment approach, using the stated preference method. Participants were given a questionnaire which is attached in the Appendix II. The questionnaire asked respondents to make a series of choices. Each choice set was assigned a cost as one attribute, and the risk of diabetes, heart disease, and stroke were the three objects to be valued. The marginal value of a one unit change in each health risk was revealed by the ratio of the attribute coefficient to the cost coefficient, which can be yielded using a logistic regression (Holmes and Adamowicz, 2003).

\section{(c) Design of the CV Survey}

An example of the WTP question in the CV survey is shown below:

\begin{tabular}{|c|c|c|c|c|}
\hline No. & \multicolumn{3}{|c|}{ Scenario } & Response \\
\hline & \multicolumn{3}{|c|}{$\begin{array}{l}\text { Would you be willing to pay } \$ 1000 \text { at one go for the pill if it } \\
\text { were to reduce risk of developing diabetes, stroke, and heart } \\
\text { disease by [amounts below] respectively in the next ten years? }\end{array}$} & \\
\hline \multirow[t]{2}{*}{$\begin{array}{l}\text { Risk Reduction } \\
\text { for... }\end{array}$} & Diabetes & Stroke & Heart Disease & \\
\hline & $26 \%$ & $3 \%$ & $4 \%$ & Yes/No \\
\hline
\end{tabular}

As the example shown above, dichotomous questions were given to participants in the survey. They were asked to make decisions about whether to pay a certain amount of money to reduce the health risks by different levels in the next ten years given various hypothetical ages and incomes. Following the main findings of the literature review in this chapter, the risks of diabetes assigned to participants were set at $7 \%, 26 \%$, or $83 \%$. The risk levels for heart disease were $4 \%, 19 \%$, and $35 \%$. The risks of stroke were determined to be $3 \%, 21 \%$, and $83 \%$. In order to ensure that the reported WTP values not only include the health harm of diseases, but also account for the loss in productivity and time cost, a sentence was written in the questionnaire to remind respondents that health problems would not only reduce quality of life, but also cause income loss and other costs as well.

The price of the hypothetical pill assigned to participants was stated to be one of eight levels: NZ \$1,000, NZ \$3,000, NZ \$5,000, NZ \$10,000, NZ \$30,000, NZ \$80,000, 
NZ $\$ 180,000$, and NZ \$382,000. The maximal WTP of $\$ 382,000$ was based on the following estimation. Since the threshold of incremental cost-effectiveness ratio (ICER) is set to approximately $\$ 40,000$ to $\$ 50,000$ per QALY in some health economics evaluation studies in New Zealand, the maximal WTP for 1 QALY (living in perfect health conditions for a year) is plausibly around NZ\$40,000. Given a $3 \%$ discount rate, the present value of living in perfect health for 10 years is estimated as $\mathrm{NZ} \$ 40,000+\mathrm{NZ} \$ 40,000 / 1.03+\ldots+\mathrm{NZ} \$ 40,000 /\left(1.03^{\wedge} 9\right)=\mathrm{NZ} \$ 381,208$. In order to test whether peoples' potential maximum WTP for the largest health risks reductions is above NZ\$382,000, as well as to identify potential problems with the CV survey, a small pilot survey was conducted amongst students who enrolled in economics courses (ECON 405, ECON 411, and ECON 339), and some PhD candidates at Victoria University of Wellington, New Zealand, during September and October 2017. Unlike the CV survey which adopted a dichotomous choice question format, an open-ended questionnaire was applied in the pilot survey. This is because open-ended format would avoid the effects of staring point bias and range bias (Klose, 1999; Stalhammar, 1996). That is, people's maximum WTP was probably not limited by the starting point and the range of given WTPs in this case. The findings of the pilot survey indicated that the maximum WTP was very unlikely to be more than NZ\$382,000.

As the possible combinations of the health risks and hypothetical WTPs were too numerous to all be surveyed, the D-optimal fractional factorial algorithm was applied to create the optimal 72 combinations, which could reveal $98.1 \%$ of the effects (efficacy $=98.1 \%$ ) of the total combinations of health risk reductions and price of the hypothetical pill. The D-optimal design is a commonly used method to reduce survey costs. Given a certain precision, this method yields the highest efficacy (Holmes and Adamowicz, 2003). The 72 different combinations and the eight versions of CV questionnaire are included in Appendix II. In order to avoid the potential problem of fatigue, each participant was randomly given twenty-seven WTP questions. This plan seemed to be feasible based on the feedback from pilot surveys.

The questionnaire also included the following variables: peoples' decisions about 
whether to pay or not, self-assessment of risk, the degree to which the diseases were known by participants, the degree to which the survey was understood, whether respondents think the public health expenditure on the diseases should be increased, and demographic information.

\section{(d) Analysis Plan}

\section{(1) Logistic Regression}

Participants' decisions (Yes / No) were analyzed with a binary logistic regression. The equation can be written as:

$$
\log \left(\frac{\mathrm{P}(\text { Yes })}{1-\mathrm{P}(\mathrm{Yes})}\right)=\beta_{0}+\sum_{\mathrm{i}=1}^{13} \beta_{\mathrm{i}} \mathrm{X}_{\mathrm{i}},
$$

where $\mathrm{P}($ Yes $)$ is the probability of being willing to pay for the hypothetical pill; $\beta_{0}$ is the intercept of the regression; $\mathrm{X}_{\mathrm{i}}$ are the following individual characteristics surveyed in the questionnaire, they are: the reduction in diabetes, stroke, and heart disease risk, price of the hypothetical pill, people's income, age, gender, educational level, ethnicity, perceived self-health risk, knowledge of the diseases, the degree to which the scenarios described in the questionnaire are understood, and whether believe public health expenditures should be increased or not. Risk reductions and price of the hypothetical pill are treated as continuous variables; the other variables in the equation are treated as categorical variables. All the independent variables were put into the regression simultaneously. In order to address the differences in demographic distributions between our sample and the New Zealand general population, weights were given to the independent variables for which an anomaly was seen (details of the weights are discussed in the results section).

The Wald test was used to test which variables had significant effects on participants' decisions. Multicollinearity was checked by calculating the Variance Inflation Factor (VIF), to ensure the parsimony of the logistic model. The fitness of the logistic regression was tested with the Cox R-squared value, McFadden R-squared value, and Nagelkerke (Cragg and Uhler) R-squared value. The statistical software R version 3.4.4 was used in the analysis; and $1 \%$ was chosen to be the significance level for all tests. 


\section{(2) Marginal WTP \& Marginal Harm}

The marginal WTP for risk reductions for diabetes, stroke, and heart diseases were estimated as $\beta_{\text {diabetes }} / \beta_{\text {price, }} \beta_{\text {stroke }} / \beta_{\text {price, }}$ and $\beta_{\text {heart disease }} / \beta_{\text {price }}$ respectively.

The marginal harm taken into account was estimated using the following method: let $\mathrm{N}_{\operatorname{lm}}$ denote light-to-medium drinkers' expected consumption of SSBs per year. The total consumption over the next ten years would, therefore, be $10 \mathrm{~N}_{\mathrm{lm}}$. If we assume that the marginal harm per litre taken into account is constant, then it can be calculated as $\left[\left(\beta_{\text {diabetes }} / \beta_{\text {price }}\right) * 7+\left(\beta_{\text {stroke }} / \beta_{\text {price }}\right) * 7+\left(\beta_{\text {heart disease }} / \beta_{\text {price }}\right) * 2\right] / 10 \mathrm{~N}_{\mathrm{lm}}$, where the numbers 7, 7, and 2 are the light-to-medium drinkers' increased risk of type- 2 diabetes, stroke, and heart disease respectively, compared with the risks of a non-drinker according to the findings of the literature review in this chapter (De Koning, 2011; De Koning, 2012; Fung et al., 2009). The value of $\mathrm{N}_{\mathrm{lm}}$ is estimated in section 4.2.2.

\section{(e) Sample Selection}

Samples were drawn from Facebook users in the 56 Facebook community groups in Wellington. The 56 groups are listed in the Appendix II. Data from some participants were dropped before the logistic regression. The selection was based on two criteria: 1) how well the scenarios were understood by the participants and 2) how much time each people spent on the survey. People who failed to understand the scenarios were thought to be more likely to give meaningless answers - they might randomly assign a YES or NO answer to each question, or give straight YES or NO answers, based on the main findings from the classroom experiment pilot survey. Therefore, those who claimed that they totally did not understand the scenarios were excluded in the analysis.

In addition, the online survey system (Qualtrics) automatically recorded the time used to finish the survey, which gave an indication of how seriously the survey was treated by each participant. Based on this, we can identify which responses were reliable and should be used in the analysis.

\section{(f) Ethical Consideration}

This research had been approved by the Victoria University of Wellington Human 
Ethics Committee (0000025927) before Facebook community administrators were contacted; and permission had been obtained from the Facebook community page administrators before the surveys were released online. Questions in the questionnaire were checked and discussed by the committee to minimize the emotional harm to participants, to protect their privacy, and to guarantee that the study is in line with the Treaty of Waitangi. Following the guidelines of the ethical approval, answers from those participants who did not complete the whole questionnaire were not used in the analysis.

\section{(g) Pilot Surveys}

In order to test the feasibility of the survey, two pilot CV surveys using students as participants were conducted in March 2018. To test the feasibility of an open-ended CV survey, a small pilot survey was conducted amongst students who enrolled in honours level economics courses. The results indicate that most people thought that open-ended format WTP questions are very hard to answer, and a dichotomous choice question format is preferred.

In the second pilot survey, participants were economic students (enrolled in the following economic courses: ECON 201, ECON 307, and ECON 340, ECON 404) and masters students as well as $\mathrm{PhD}$ candidates at Victoria University of Wellington, New Zealand, 2018. Unlike the first pilot survey, a dichotomous choice question format was adopted. Paper questionnaire sheets were delivered to the students in lectures, and they could decide whether to take part in the survey or not. In order to encourage potential participants to take part in the survey, Cadbury chocolate was provided as rewards. In addition, informed consent was obtained from participants before the survey. All the finished questionnaires were destroyed on March $28^{\text {th }}, 2018$, to ensure that participants' personal information would not be released.

Because the sample in this pilot survey had limited diversity in age and income, hypothetical income and age were also assigned to the participants in the second pilot survey. Based on the income distribution in NZ, hypothetical annual income was set at one of four levels: NZ\$30,000, NZ\$90,000, NZ\$150,000, and NZ\$200,000. Hypothetical ages in this survey were determined to be 25,45 and 65 . This design 
goes someway to protecting participants' privacy, as their real incomes and ages were not asked. Since the possible combinations of the health risks, hypothetical income, and hypothetical age are too numerous (2,592 combinations) to all be surveyed, the D-optimal fractional factorial algorithm was applied to create the optimal 96 item choice set, which could reveal $96.4 \%$ of the effects (efficacy $=96.4 \%$ ) of the total combinations of health risk reductions, hypothetical income, cost of the hypothetical pill, and hypothetical ages. In order to avoid the potential problem of fatigue, each participant was randomly given only six WTP questions. In addition, the pilot survey also surveyed SSB consumption per month which may be different from the general population. The average of students' SSB consumption was used in the estimation of the harm from SSBs for this specific group. The findings of the second pilot survey are briefly reported in the results section. The ethical approval of the pilot survey was received on September 22, 2017 (Ethics Approval: 25322).

In order to test the feasibility of the survey on Facebook, another pilot CV survey was conducted in Pharmaceutical Management Agency (PHARMAC) and Upper Hutt library in May 2019. This pilot survey suggested that the design was reasonably acceptable to the New Zealand general population.

\subsubsection{Sensitivity Analysis}

In order to deal with uncertainty in SSB consumption due to household size, for household size $=8$ or $>8$, the average number of people per household was changed to 12 (increased by $50 \%$ ) in the sensitivity analysis. This modification changed the weighted average number of people per household to 3.62, and SSB consumption per person per year decreased to 68.36 litres.

In order to deal with uncertainty in disease risks, the diabetes risk, stroke risk, and heart disease risk for light-to-medium drinkers were changed to $9 \%, 12 \%$, and $4 \%$ respectively based on the findings of the literature review in this chapter (De Koning, 2011; De Koning, 2012; Fung et al., 2009).

\subsection{Results}

\subsubsection{The Pilot Survey Using Students}

The pilot survey in the classroom yielded 121 valid responses (response rate $=$ 
95.28\%). 114 of them did not miss any question in the questionnaire. Since the missing values were relatively few, those respondents who had missing values were simply dropped.

A logistic regression was applied to analyze the determinants of participants' choice and of the marginal WTP for health risk reductions. Based on the coefficients estimated in the logistic regression, the marginal WTP for a $1 \%$ reduction of diabetes risk, stroke risk and heart disease risk are \$886.05, \$2,600.11, and \$2,397.25, respectively. The three health risk reductions, the price of the hypothetical pill (or WTP for the hypothetical pill), hypothetical income, gender, hypothetical age, perceived self-risks and educational levels have significant effects at the $10 \%$ significance level. Given the information reported above, when the marginal harm that is accounted for is constant, the estimation indicates that it is approximately $\$ 64.22$ per litre. Finally, the average consumption for total participants is $4,108.41 \mathrm{ml}$ per month. Details of the information mentioned in this section are reported in Table 4.2 in Appendix.

\subsubsection{The Survey on Facebook}

The online survey was put in the Facebook community group page from June $5^{\text {th }}$ to December $7^{\text {th }}, 2019$. Many Facebook community page administrators only allowed repeat advertising once per week, and some of them only allowed once per month. On some occasions, advertisements were automatically identified as a scam by Facebook. As a result, the number of individuals per community group was quite low, which was approximately 9 participants on average, and around 500 in total. However, around 110 of them stopped at the information sheet page; and another 70 stopped at, or before the WTP questions page. Since the answers from the people who stopped halfway were not recorded and returned by Qualtrics, they were not included in analysis.

A total of 305 final responses were recorded and returned from the formal survey. Given the length of the questionnaire and the complexity of the scenarios, it was thought to be impossible to finish the survey in two minutes with serious deliberation about the scenarios described in the questionnaire. Thus, participants who finished the 
survey in 2 minutes were excluded from the analysis. Of the 305 responses, 11 individuals finished in 2 minutes (9 of the 11 respondents declared in the questionnaire that they failed to understand the scenarios), and one extra participant claimed that he or she did not understand the scenarios at all. Hence, their answers were excluded from the analysis, leaving 293 valid responses. Of the 293 selected participants, a few skipped one or two WTP questions. This was thought because of carelessness, rather than being unwilling to answer the questions because all of them finished the questionnaire. Therefore, the questions they completed were included in the analysis.

Table 4.3: Summary Statistics of Variables and Missing Data for the Survey on Facebook

\begin{tabular}{|c|c|c|c|}
\hline Variable & & No. $(\%)$ & Missing Data \\
\hline \multirow[t]{2}{*}{ Decision } & Yes & $5,897(75 \%)$ & 279 \\
\hline & No & $1,735(25 \%)$ & \\
\hline \multirow[t]{2}{*}{ Gender } & Male & $49(17 \%)$ & 0 \\
\hline & Female & $244(83 \%)$ & \\
\hline \multirow[t]{5}{*}{ Race } & White & $184(63 \%)$ & 0 \\
\hline & Maori & $37(13 \%)$ & \\
\hline & Pasifika & $8(3 \%)$ & \\
\hline & Asian & $43(14 \%)$ & \\
\hline & Other & $21(7 \%)$ & \\
\hline \multirow[t]{4}{*}{ Understanding } & Totally agree & $157(54 \%)$ & 0 \\
\hline & Somewhat agree & $99(34 \%)$ & \\
\hline & Neither agree nor & $19(6 \%)$ & \\
\hline & $\begin{array}{l}\text { disagree Somewhat } \\
\text { disagree }\end{array}$ & $18(6 \%)$ & \\
\hline \multirow[t]{7}{*}{ High self-risk } & Totally agree: & $46(16 \%)$ & 0 \\
\hline & Somewhat agree & $76(26 \%)$ & \\
\hline & Neither agree nor & $39(13 \%)$ & \\
\hline & disagree Somewhat & $88(30 \%)$ & \\
\hline & disagree & $40(14 \%)$ & \\
\hline & Totally disagree: & $4(1 \%)$ & \\
\hline & Prefer not to say & & \\
\hline \multirow[t]{6}{*}{ More Government Spending } & Totally agree & $126(43 \%):$ & 0 \\
\hline & Somewhat agree & $103(35 \%):$ & \\
\hline & Neither agree nor & $39(13 \%):$ & \\
\hline & disagree Somewhat & $16(5 \%):$ & \\
\hline & disagree & $9(3 \%)$ & \\
\hline & Totally disagree & & \\
\hline
\end{tabular}


Educational Level

$\begin{array}{ll}2^{\text {nd }} \text { education or below } & 57(19 \%) \\ \text { Certi and Dip } & 76(26 \%) \\ \text { Bachelor's } & 84(29 \%) \\ \text { Postgraduate } & 61(21 \%) \\ \text { Prefer not to say } & 15(5 \%)\end{array}$

Income

$\begin{array}{ll}\text { Below } \$ 25,000 & 57(19 \%) \\ \$ 25,000 \text { to } \$ 70,000 & 129(44 \%) \\ \$ 70,000 \text { to } \$ 120,000 & 57(19 \%) \\ \text { Above } \$ 120,000 & 19(6 \%) \\ \text { Prefer not to say } & 31(11 \%)\end{array}$

Good Knowledge of the Diseases

Totally disagree

$0(0 \%)$

0

Somewhat disagree

$24(8 \%)$

Neither agree or nor disagree

$24(8 \%)$

Somewhat agree

$134(46 \%)$

Totally agree

$111(38 \%)$

Age

Below 25

$33(11 \%)$

25 to 45

$161(55 \%)$

46 to 65

$89(30 \%)$

Above 65

$8(3 \%)$

Prefer not to say

$2(1 \%)$

The descriptive statistics discussed are shown in Table 4.3 above. It should be noted that, there were 244 females and only 49 males in the sample.

A logistic regression was applied to analyze the determinants of participants' choice and the marginal WTP for health risk reductions. Since there were far more females than males in the survey, weights (female:male $=0.63: 2.82$ ) had to be given to address this anomaly.

As shown in Table 4.4 in appendix, the three health risk reductions, the price of the pill, age, perceived self-risks, how well the scenarios described in the questionnaire were understood by participants, race, and the degree to which participants were familiar with the diseases, and educational levels had significant effects at the 0.01 significance level. In particular, a $1 \%$ increase in diabetes, stroke, and heart disease risk reductions are predicted to increase the $\log$ odds $\left(\log \frac{P 1 /(1-P 1)}{P 2 /(1-P 2)}\right)$ by $0.006,0.012$ and 0.018 respectively; Every additional $\$ 1,000$ increase in the pill price would lower 
the log odds by 0.015 .

Since none of the VIF values is above 10, it might be concluded that multicollinearity is not seen in the regression. The Cox and Snell R-squared, McFadden R-squared, and Nagelkerke (Cragg and Uhler) R-squared are 0.170, 0.230, and 0.300, respectively.

Table 4.5: Marginal Harm of SSB per Litre

\begin{tabular}{lll}
\hline SSB Consumption per Person Per Year & $\mathbf{7 0 . 7 1}$ Litres & 68.36 Litres \\
\hline Base Case Disease Risks & $\$ 15.52 /$ litre & $\$ 16.05 /$ litre \\
Sensitivity Analysis Disease Risks & $\$ 25.88 /$ litre & $\$ 26.78 /$ litre \\
\hline
\end{tabular}

Table 4.5 above shows estimated marginal harm. Given the coefficients estimated in the logistic regression, the marginal WTP for a $1 \%$ reduction in diabetes risk, stroke risk and heart disease risk are $\$ 404.86, \$ 809.04$, and $\$ 1,236.84$, respectively. According to the method described previously, when SSB consumption per person per year is 70.71 litres, the estimation indicated that the marginal harm considered would be approximately $\$ 15.52$ per litre.

The sensitivity analysis showed that when SSB consumption per person per year is 68.36 litres, the estimation indicates that the marginal harm taken into account is approximately $\$ 16.05$ per litre. When the diabetes risk, stroke risk, and heart disease risk for light-to-medium drinkers are changed to $9 \%, 12 \%$, and $4 \%$ respectively, the marginal harm taken into account would increase to approximately $\$ 25.88$ per litre. If both the SSB consumption per person per year and risks are changed at same time, the considered marginal harm would go up to approximately $\$ 26.78$ per litre. The results of the sensitivity analysis might indicate that the risks used in estimation could affect the results by a large margin.

\subsection{Discussion}

\section{(a) Limitations}

The limitations of this study included the following. First, the results of the contingent valuation survey may be affected by hypothetical bias. It is not clear whether the amounts stated by participants were their real WTPs or not, as they did not really need to pay money to buy the hypothetical pill (Goodstein, 2011). Based on the findings of a meta-analysis that the mean ratio of hypothetical to actual was 2.6 (Murphy et al, 
2006), the marginal WTPs in the study are thought to be highly likely to be overestimated. As a result, the marginal harm of SSBs from internalities is probably overestimated due to hypothetical bias.

Furthermore, in one of the two meta-analysis studies in which intensive drinkers were studied, the observational duration was 10.96 years, while in the two selected studies in which light-to-medium drinkers were concentrated, the durations were around 20 years. But in our survey, the effect of the hypothetical pill was set to be 10 years. Given this, the marginal WTPs and harm from SSBs from internalities are probably overestimated again. This concern is reinforced by the sensitivity analysis, which showed that the risks used in estimation might affect the results by a large margin.

All in all, it may be reasonable to conclude that the marginal WTPs and harm of SSBs from internalities are overestimated. However, it should also be noticed that the harm of dental problems or even cancer from excess SSB consumptions were not included in this study. As a result, there is also a risk that the marginal harm of SSB from internalities can be underestimated. Having had taken all these factors into consideration, whether the results are overestimated or not cannot be determined.

Another factor which may cause our results biased is order bias. Although different versions of questionnaires were allocated randomly to participants, their decision-making still can be biased as people may always refer the values in the scenarios described in the previous question. Some studies suggest that this problem probably can be addressed by changing the WTP questions to an open - ended format (Boyle, Welsh, \& Bishop, 1993). The open-ended format was originally considered in this study. However, a dichotomous choice question format was finally chosen, because almost all the respondents claimed that dichotomous format was much easier to answer in the pilot survey. If participants found the open-ended format very difficult to answer, they would probably be much more likely to give a meaningless answer, or refuse to answer, which would make the sample smaller. Given these arguments, it seems quite challenging to find a solution to get rid of the order bias, while yields a reasonably high response rate and an acceptable sample size.

Also, it should be noted that more than $20 \%$ of the participants who began the survey 
did not finish the whole questionnaire. Since their answers were not recorded by Qualtrics, it was impossible to know how the results were affected by those who omitted answers. Hence, there is a probability of omitted bias.

In addition, people's decisions depended on how severe the participants think the diseases were, but little detail about the severity of the diseases was provided in the questionnaires. The reason why a more detailed description was not provided was that a long questionnaire was considered burdensome for participants and may result in a low response rate. Hence, whether the WTP values revealed the average level of severity or other levels was still impossible to know.

It should also be noted that participants may misunderstand the risk reductions described in the questionnaire. In the scenarios, risk reductions were framed as a reduction by a certain percent, rather than a reduction by pure percentage points. This was partly because the increased risk from SSB consumption was revealed by hazard ratios in the selected literature; and hazard ratios are conveniently interpreted as a reduction by a certain percent, rather than pure percentage points. In addition, because different people have different self-risks, we believe that providing an average baseline risk is somewhat useless. Given all these arguments, we decided to reframe the risk reductions as a reduction by a certain percent and include the self-risk as an independent variable in the logistic regression. However, if participants had incorrectly assumed that the questions were referring to absolute risk reductions, the WTPs estimated in this study would be substantially overestimated (approximately 58 times larger).

Another controversial question could be whose perspective should be adopted, or rather, who should be included in the sample? It could be questioned whether university students, or even the general population, can understand diabetic and cardiovascular disease patients' suffering. One possibility is that if the participants were replaced by the real patients or healthcare workers, the marginal WTP for health risk reductions may become much larger. But, if real patients were surveyed, it could also be questioned whether they would exaggerate their WTP to influence the health policy decision-makers to increase health investment on them. There may, therefore, 
be strategic bias in such responses.

Besides, our estimation was made under the assumption of constant marginal WTP for each percentage of risk reduction. However, there is still insufficient evidence to verify this assumption.

Finally, given that only some of the Facebook users in the community groups were surveyed, it should be questioned that whether the findings of this study can apply to New Zealand general population. Even if we limit it to Facebook users in Wellington area, the conclusions are still questionable, as there are too many females in the sample, and the distribution surely deviates from gender ratio of New Zealand population. One possible explanation of the unbalance in gender could be that females are probably more willing to help others, and more interested to health - related topics.

\section{(b) Strengths}

One strength of the study is that the WTP values include not only the health harm of diseases, but also potentially count other costs such as the loss in productivity, time costs, and emotional harm of relatives and friends. Moreover, compared with other CV studies, the valid response rate was quite high in this survey. Finally, this study is probably the first one that has tried to estimate the monetary value of the marginal harm of SSBs by applying CV.

\subsection{Conclusion}

This CV study investigated 293 Facebook users in Wellington and found that the marginal WTP for a $1 \%$ reduction in diabetes risk, stroke risk and heart disease risk is \$404.86, \$809.04, and \$1,236.84, respectively. Given SSB consumption per person per year, the marginal harm considered is approximately $\$ 15.52$ per litre, with a likely range from $\$ 15.52$ to $\$ 26.78$ per litre. Sensitivity analysis showed that the risks used in estimation could affect the results by a large margin. Our results are thought to be with some uncertainty and limited generalizability. 


\section{Measuring the Harm of SSBs Associated with Externalities}

\subsection{Introduction \& Objective}

In order to answer the research questions 1 to 4 , a theoretical model of the optimal corrective tax was constructed in chapter 3. Recall that the optimal-tax formula is:

$$
\tau=\left[(1-\beta) H_{I}+H_{E}\right] \frac{\sum_{j=1}^{N} r_{j} \frac{d q_{j}}{d \tau}}{\sum_{j=1}^{K} b_{j} \frac{d q_{j}}{d \tau}},
$$

where $H_{I}$ is the marginal health harm of SSB consumption, $\beta \in(0,1)$ represents the proportion of the harm considered by consumers, so that $(1-\beta) \mathrm{H}_{\mathrm{I}}$ is regarded as internalities. $\mathrm{H}_{\mathrm{E}}$ is the marginal cost of externality from SSB consumption, and $\frac{\sum_{j=1}^{N} r_{j} \frac{d q_{j}}{d \tau}}{\sum_{j=1}^{K} b_{j} \frac{d q_{j}}{d \tau}}$ is the multiplier.

Since the marginal cost of externalities from SSB consumption, $\mathrm{H}_{\mathrm{E}}$, is included as a component of the optimal tax formula, its monetary value has to be estimated. As discussed in the literature review section, the costs from externalities have been estimated by Allcott, Lockwood, and Taubinsky (2019). However, because of the heterogeneity in healthcare systems cross countries, their findings are very unlikely to be applicable outside the US. Therefore, there is a need to measure the monetary value of externalities from SSB consumption in a New Zealand context.

As noted in the previous chapters, an increased risk of diabetes and cardiovascular diseases (CVDs) is probably associated with excess SSB consumption. Since the New Zealand public health system is largely funded by taxpayers, these costs can be viewed as fiscal externalities. Given this, we can estimate the harm of SSBs associated with externalities by assessing the lifetime cost of diabetes, stroke, and ischaemic heart disease (IHD). The methods used to measure $\mathrm{H}_{\mathrm{E}}$ are discussed below.

\subsection{Method}

If we assume the marginal harm of externalities from SSB consumption is constant, 
then it will be equal to the average harm from externalities which can be calculated as the total harm from externalities divide the total SSB consumption in a certain period. As discussed in the first chapter, an association between SSB consumption and certain diseases has been identified in a few studies. The more SSBs people consume, the higher is the risk they will develop diseases. Consequently, the total costs of externalities probably can be regarded as the increase in medical expenditures paid by the government, and the increase in medical expenditures probably can be calculated as the lifetime treatment costs of diseases multiplied by the increase in disease risks. For example, if the lifetime treatment costs of a disease are $\$ 1,000$, the risk of developing the disease is $1 \%$ for a non-drinker and 3\% for a light-to-medium drinker, then the total costs from externalities are $\$ 1,000 *(3 \%-1 \%)=\$ 20$. If the average SSB consumption for a light-to-medium drinker is 1000 litres, then the marginal cost from externalities is estimated to be $\$ 20 / 1000=\$ 0.02 /$ litre.

Let $\mathrm{H}_{\mathrm{d}}$ demote the marginal/average harm associated with an IHD, or a stroke, or diabetes, $C_{d}$ denote the lifetime healthcare costs of these diseases paid by the New Zealand healthcare system, $\mathrm{R}_{\mathrm{o}}$ denote the risk of developing such diseases for an average person who is assumed to be a light-to-medium drinker, $R_{n}$ is the risk of developing an IHD, or a stroke, or diabetes for a non-drinker, and $\mathrm{N}_{\text {ssb }}$ denote the average SSB consumption for a light-to-medium SSB drinker. Then, the relationship between the five terms is:

$$
\mathrm{H}_{\mathrm{d}}=\frac{\mathrm{Cd} *(\mathrm{Ro}-\mathrm{Rn})}{\mathrm{Nssb}}
$$

In order to calculate the value of $\mathrm{H}_{\mathrm{d}}$, the three components on the right have to be estimated. The methods used to measure each component are explained in the next few sections.

\subsection{Costs}

\subsubsection{Source of Cost Data}

This section discusses the method used to measure the costs associated with diseases, $\mathrm{C}_{\mathrm{d}}$. Hospital inpatient costs are sourced from the New Zealand Ministry of Health inpatient dataset. Inpatient costs are calculated using the diagnosis-related groups 
(DRGs) released on the Ministry of Health website. ${ }^{4}$ DRGs are a patient classification system which relates the number of types of patients treated in a hospital to the resources required by the hospital. DRG costs cover all the resources used for patient care in the hospital, from hospital admission to hospital discharge (except oncology diagnoses and procedures). Average DRG costs are calculated from cost-weight unit DRG price and the inlier cost-weight of each item. The 2018 / 2019 cost-weight unit price was $\$ 5,068.12$.

Outpatient and laboratory test costs are estimated based on the unit purchase price (PU) and average units required by each item. They are sourced from the Ministry of Health website and the 2018 cost manual spreadsheet from Pharmaceutical Management Agency (PHARMAC).

Finally, the drugs used for each disease are based on the New Zealand primary care handbook (New Zealand Primary Care Handbook 2012, 2012). Drug costs are calculated based on the price of each drug which can be found on the PHARMAC Online Schedule. Dosages of the drugs are sourced from the guideline and the Medsafe website.

\subsubsection{Costing Method}

'Bottom-up' and 'top-down' methods are used respectively to estimate the costs of a stroke and a heart disease per year depending on which type of data is available. The 'bottom-up' method uses patient level data to calculate costs, while the 'top-down' method uses aggregated data. When the 'bottom-up' method is applied, costs of complications are further divided into different categories. They are hospital costs (inpatient and outpatient), allied health professional costs, residential or aged care costs, and pharmaceutical costs, as well as laboratory tests. In addition, since several the diseases included may cause sudden death, the costs of each disease in the first year are broken into costs of a fatal event or a non-fatal event, and the costs of the disease in the first year are calculated as the weighted average of the two. The allied health professional costs and pharmaceutical costs depend on the healthcare services

\footnotetext{
${ }^{4}$ www.health.govt/nz-health-statistics/data-references/weighted-inlier-equivalentseperations
} 
and medicine used to treat each disease; and the healthcare services and medicine used to treat each disease are based on the experts' opinion from PHARMAC and medical guidelines such as the New Zealand primary care handbook (New Zealand Guidelines Group, 2012). All costs are adjusted for inflation and expressed in NZ dollars for the year 2019 .

The costing methods described are used to calculate the annual costs associated with a stroke, an IHD, or type-2 diabetes. Given the annual costs of a stroke and an IHD, the lifetime costs of these two diseases are estimated using a Markov model. The lifetime costs of type-2 diabetes are estimated using the UK Prospective Diabetes (UKPDS) model. In order to deal with uncertainty in variables, sensitivity analysis is applied to estimate the possible range of results. Details are discussed below.

\subsubsection{Costs Associated with a Stroke}

Stroke is the third most important cause of death, and the eighth highest cause of health loss in New Zealand. It accounted for approximately $8.2 \%$ of all deaths and $4.2 \%$ of premature deaths in 2018, and generated heavy economic and social burden to patients, families, and New Zealand healthcare systems (NZIER, 2018). Studies have found that in addition to the large costs of healthcare services, many stroke survivors may become disabled; they may have one or more physical, mental, or cognitive problems after a stroke, and these health problems often require long term medical interventions, residential care, and informal care (NZIER, 2018). It has been estimated that 9,500 stroke incidences caused more than $\$ 880$ million social and economic loss (including the loss in quality of life and premature death) in New Zealand in 2017, and each stroke was associated with $\$ 60,000$ to $\$ 99,000$ loss over five years on average (NZIER, 2018).

Given the information above and the costs of a stroke identified in The Social and Economic Costs of Stroke in New Zealand (NZIER, 2018), the costs of stroke included in our cost model are classified as: (1) hospitalization, (2) rehabilitation and ongoing support (including long term residential care and informal care), (3) longer term medical costs, and (4) outpatient general practitioner visits and laboratory costs. Each item is estimated below. 


\section{(1) Hospitalization Costs}

The cost associated with a stroke hospitalization event in the first year (non-fatal) is calculated by taking the arithmetic average of the stroke DRGs (DRG B70A, DRG B70B, and DRG B70C). These DRGs include the treatment costs of a stroke, from the most serious one with catastrophic complications to the least serious one without any complication. The cost associated with a stroke hospitalization event in the first year (fatal) is calculated based on the stoke DRG B70D. Details are reported in Table 5.27 in Appendix.

(2) Rehabilitation Costs

Rehabilitation costs include both hospitalization and outpatient costs. The rehabilitation hospitalization cost is calculated based on the rehabilitation DRG (DRG Z60Z). Based on experts' opinion, rehabilitation hospitalization is assumed to occur only in those most serious patients with catastrophic complications after a non-fatal stroke.

The rehabilitation outpatient costs are calculated based on the average number of Purchase Unit (PU) each rehabilitation costs. According to experts' opinion from PHARMAC, stroke survivors are assumed to receive outpatient rehabilitation treatments once per month during the first year after hospital discharge.

(3) Residential Care

Residential care costs are calculated based on the information below. The total residential care for 299 stroke survivors cost \$8,119,632 in 2018 (NZIER, 2018). This implies that the residential care cost per patient per year were approximately $\$ 27,155.96$ in 2018 .

In the base case, we assume that additional residential costs are attributed to $10 \%$ of all stroke survivors. This estimation is based on the following evidence. First, the National Acute Stroke Services Audit, 2009 shows that $90 \%$ of the stroke patients are living in their own homes prior to the stroke, so the rest $10 \%$ are living in residential care (NZIER, 2018). Second, it is thought that those survivors who have had been living in residential care would return to residential cares after hospital discharge. Third, $20 \%$ of stroke survivors are admitted to residential care after hospital discharge. 
Based on the information listed above, the additional number of residents after a stroke is the difference between the number of survivors are admitted to residential care after hospital discharge ( $20 \%$ of survivors) and the number of those people who had been living in residential care prior to the stroke (10\% of survivors). Therefore, the expected cost of residential care per patient per year would be $\$ 2,715.60$ in 2018 . Given this, the costs of residential care per patient per year were estimated to be $\$ 2,756.33$ in 2019 after being adjusted for the inflation rate.

(4) Informal Care Costs

The average duration of informal care for a stroke survivor is 427.48 hours per patient per year in New Zealand (Claesson et al., 2000). Given the female median hourly wage ( $\$ 23.75$ per hour in 2018 ) and the fact that caregivers are more likely to be females, the costs of informal care were estimated to be approximately $\$ 10,304.94$ per patient per year in 2019, after being adjusted for the inflation rate.

(5) Long Term Medical Costs

According to the New Zealand primary care handbook (New Zealand Guidelines Group, 2012), the pharmaceutical costs for a stroke include spending on Cilazapril with hydrochlotothiazide, a statin, Clopidogrel, and Aspirin. In the base case, the dosage of Cilazapril with hydrochlotothiazide, Clopidogrel, a statin, and aspirin are $5 \mathrm{mg}, 75 \mathrm{mg}, 30 \mathrm{mg}, 4 \mathrm{mg}$, and $100 \mathrm{mg}$ per day, respectively. In the sensitivity analysis, the dosages are changed to the higher and lower bound of the suggested amounts in the New Zealand Primary Care Handbook (New Zealand Guidelines Group, 2012). Details of the unit price, dosage, and costs for each drug are reported in Tables 5.1 to 5.3 in Appendix.

Other non-pharmaceutical costs include neurologist visits and blood tests (FBC). According to experts' opinion, an average patient is assumed to have two neurologist visits and two blood tests per year. In the sensitivity analysis, the costs of neurologist visits and blood tests are excluded in the most conservative situation as these costs are considered inapplicable to those patients who are fully recovered a year after a stroke. As shown in Table 5.3 below, the costs of a non-fatal and a fatal stroke (died in 30 days) are approximately $\$ 30.926 .20$ and $\$ 3,470.02$ respectively in the first year. 
According to the report that 1 in 8 strokes are fatal within the first 30 days (NZIER, 2018 ), the expected cost for a stroke in the first year is approximately $\$ 27,494.18$, and the annual costs for a non-fatal stroke are $\$ 14.019 .08$ in subsequent years.

Table 5.3.1 Costs of Stroke: Base Case (Reduced Table)

\begin{tabular}{|c|c|c|c|c|}
\hline & \multicolumn{2}{|c|}{ Stroke } & \multicolumn{2}{|c|}{ Non - DRG Costs } \\
\hline & Fatal & Non-Fatal & & Total annual costs \\
\hline \multirow{11}{*}{$\begin{array}{l}\text { Hospitalization } \\
\text { Costs ( DRG) }\end{array}$} & $\$ 3,470.02$ & $\$ 14,314.00$ & Cilazapril with & $\$ 39.42$ \\
\hline & & & hydrochlotothiazide & \\
\hline & & & Clopidogrel & $\$ 23.64$ \\
\hline & & & Statin & $\$ 10.94$ \\
\hline & & & Aspirin & $\$ 3.98$ \\
\hline & & & Neurologist Outpatients & $\$ 862.47$ \\
\hline & & & Full blood count & $\$ 17.36$ \\
\hline & & & Residential care cost & $\$ 2,756.33$ \\
\hline & & & Informal Care costs & $\$ 10,304.94$ \\
\hline & & & Total & $\$ 14,019.08$ \\
\hline & & & Rehabilitation ( $1^{\text {st }}$ year only) & $\$ 2,593.12$ \\
\hline Annual & $\mathbf{0}$ & $\$ 14,019.08$ & & \\
\hline \multicolumn{5}{|l|}{ Cost after year 1} \\
\hline $\begin{array}{l}\text { Total Cost at in } \\
\text { Year } 1\end{array}$ & $\$ 3,470.02$ & $\$ 30.926 .20$ & & \\
\hline
\end{tabular}

(6) Lifetime Costs of a Stroke

Stroke survivors have a risk of dying each year after the event. Therefore, a probabilistic two-state transition Markov model using annual cycles is constructed to explore the proportion of stroke survivors who are still alive, and the costs associated with a stroke through patients' lifetime.

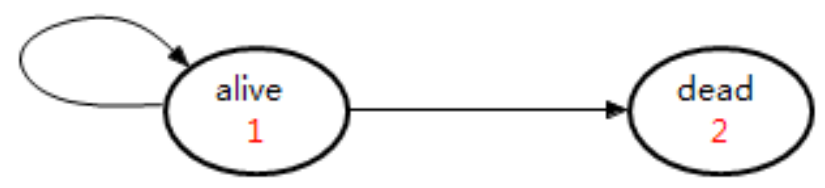

Figure 5.1: Markov Chain for a Stroke Survivor

As shown in Figure 5.1 above, there are two health states: "alive", and "die" in the Markov model. All stroke survivors start in the 'Alive' state. In each cycle, they can either stay alive, or die, with a mortality rate. Costs are accrued in each cycle of the model while the patients are in the 'Alive' state. Therefore, the lifetime costs can be calculated as: 


$$
\sum_{i=1}^{40} c_{i} P_{i}
$$

where $c_{i}$ is the costs in the year $i$, and $P_{i}$ is the proportion of people who survive in that year. In order to ensure that the model covers patients' entire lifetime, the time horizon of the model (the number of years simulated) is set to be 40 years. This is based on the information that the average age of New Zealand population is 38 years and expected life expectancy is 82 years. In the Markov model, all costs are discounted at $3.5 \%$ per annum according to the Prescription for Pharmacoeconomic Analysis (PFPA).

$\mathrm{P}_{\mathrm{i}}$, the proportion of people who are alive in each year, is calculated from two Kaplan-Meier survival curves. (1) The Kaplan-Meier survival curve in the first year is extracted from the Social and Economic Costs of Stroke (NZIER, 2018). According to this report, one in eight strokes is fatal within the first 30 days, and one in four strokes are fatal within a year. Therefore, the probability of survival is assumed to be 0.875 and 0.750 for one month and one year after the event respectively. (2) The proportion of people who are alive from year one to year eleven is extracted from the survival curve reported in Jennum et al.'s study (2015).

As shown in Figure 5.4 below, having plotted the proportion of people who are alive on a graph, an exponential regression is estimated. The regression function is then used to predict the proportion of people who are alive after year eleven. Given the proportion of people who are alive in each year, the expected costs in the first year, and the annual costs in subsequent years, lifetime total costs associated with a stroke are estimated to be approximately $\$ 87,166.35$ after discounting.

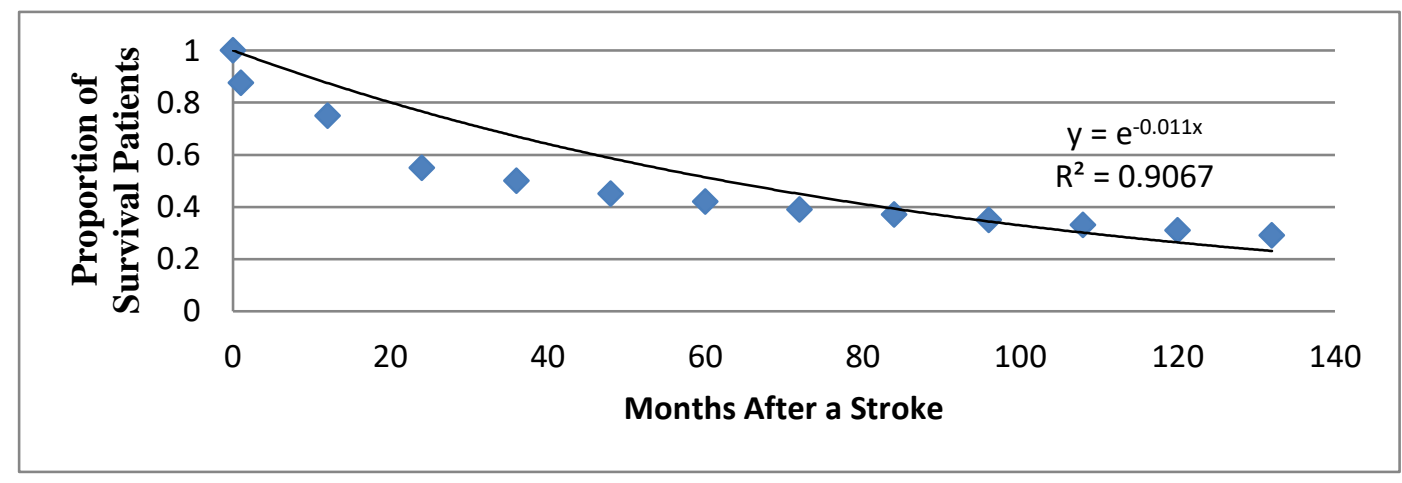

Figure 5.2 Proportion of Survival after a Stroke

In order to deal with uncertainty, one-way deterministic sensitivity analysis is applied 
in this study. In this sensitivity analysis, the probability of survival for a stroke survivor from year one to year twelve is extracted from the other survival curve which only accounts for the proportion of patients who are survived after a cerebral haemorrhage, rather than all stroke patients (Jennum et al. 2015). Details are shown in Figure 5.5 in Appendix.

\subsubsection{Costs of an IHD}

Amongst all deaths in New Zealand, the leading cause is ischaemic heart disease (IHD). Statistics have shown that $26 \%$ of IHD patients are aged below 75 in New Zealand, and males are more likely to die from IHD than females (Ministry of Health, 2014; Grey et al., 2018). As with stroke, the economic burden of IHD is quite heavy. A report to the Ministry of Health revealed that 30,745 IHD hospital admissions imposed over NZ\$228 million of costs to the New Zealand healthcare system in 2011 (National Health Committee, 2013).

(a) Costs in the year of the event

A top-down method is used to calculate the costs in the year of an IHD. Statistic indicated that there were 21,764 coronary/ischemic heart disease (IHD) hospitalization admissions, and total expenditures on IHD were approximately $\$ 228$ million in the year 2013 (National Health Committee, 2013). Given this, the average annual costs of an IHD in the first year are estimated to be approximately $\$ 11,241.10$ in 2019 (hospitalization plus treatments after discharge), after being adjusted for inflation.

(b) Annual costs in subsequent years

The costs per year after discharge are estimated based on the treatments suggested in the guideline. The suggested interventions following an IHD include Cilazapril with hydrochlotothiazide, Metoprolol, a statin, Marevan and Aspirin (New Zealand Guidelines Group, 2012). In the base case, the dosages of Cilazapril with hydrochlotothiazide, Metoprolol, statin, Marevan, and Aspirin are 5mg, 75mg, 30mg, $4 \mathrm{mg}$, and $100 \mathrm{mg}$ per day respectively. In the sensitivity analysis, the dosage for metoprolol, marevan, and a statin are changed to the higher and lower bounds of the suggested amounts in the guideline (New Zealand Guidelines Group, 2012). 
Table 5.6 Non-DRG Costs of IHD after year 1: Base Case (Reduced Table)

\begin{tabular}{|c|c|}
\hline Non-DRG Costs & Total annual costs \\
\hline Cilazapril with hydrochlotothiazide & $\$ 39.42$ \\
\hline Metoprolol & $\$ 30.99$ \\
\hline Statin & $\$ 10.94$ \\
\hline Marevan & $\$ 110.96$ \\
\hline Aspirin & $\$ 3.98$ \\
\hline Cardiacology & $\$ 868.77$ \\
\hline Full blood count & $\$ 26.04$ \\
\hline & $\$ 1091.10$ \\
\hline
\end{tabular}

The costs associated with an IHD (base case) are summarized in the Table 5.6 above. Our calculation implies that the annual costs of IHD are approximately $\$ 1,090.10$ in subsequent years. More details of the unit price, dosage, and costs for each drug in the base case and the sensitivity analysis are reported in Tables 5.4 to 5.6 in Appendix.

(c) Lifetime costs

Lifetime costs for an IHD are also estimated using a probabilistic two-state transition Markov model which includes the probability of survival and costs. The probability of survival after a cardiovascular event is calculated based on the information below. According to Statistics NZ, there were 18,513 IHD hospital admissions and 5,099 deaths related to IHD respectively in 2014 (Grey et al., 2018). Therefore, the proportion of people who are alive after the event can be calculated as $(18,513-5,099)$ $/ 18,513=0.725$

The proportion of people who are alive after a cardiovascular event from year one to year five is extracted from the Kaplan Meier survival curve reported in Wong, Tang, and Herbison (2007). This curve shows the proportion of survivors with acute coronary syndrome discharged from a tertiary teaching hospital in Dunedin, New Zealand. Having plotted the figures in a graph, an exponential regression is estimated. The function of the regression is then used to predict the probability of survival in each year after the year five. Details are shown in Figure 5.6 in Appendix.

In order to deal with uncertainty, one-way deterministic sensitivity analysis is applied. In the sensitivity analysis, the probability of survival after a cardiovascular event from year one to year five is also extracted from the Kaplan Meir survival curve reported in Wong, Tang, and Herbison (2007). This curve shows the proportion of survivors with 
acute coronary syndrome discharged from a different hospital - the tertiary teaching hospital in Invercargill, New Zealand. Details are shown in Figure 5.7 in Appendix.

\subsubsection{Diabetes}

Type-2 diabetes is a chronic disease characterized by high blood sugar levels (hyperglycemia). It is a result of insufficient insulin production, the hormone regulates blood sugar levels, or of an ineffective response to the insulin the body produces (New Zealand Guidelines Group, 2012). According to the Virtual Diabetes Register of New Zealand in 2018, there were approximately 250,000 diabetic patients in New Zealand, and $90 \%$ of these registrations were for type- 2 diabetes. ${ }^{5}$

It should be noted that diabetes is associated with severe long-term consequences. Microvascular consequences include peripheral neuropathy, diabetic retinopathy, and nephropathy. Macrovascular consequences include ischemic cardiovascular disease, stroke and heart failures (New Zealand Guidelines Group, 2012). The risk of developing these complications is reduced with good blood pressure, blood glucose and blood cholesterol control, but increased with diabetes duration (New Zealand Guidelines Group, 2012).

\subsubsection{The UKPDS Model}

Unlike stroke and IHD, there have been a few widely used economic models for diabetes. In this study, the costs of diabetes are calculated using the UK Prospective Diabetes Study (UKPDS) model which was developed based on the UKPDS Study (UKPDS). The study is one of the most well-known and longest clinical diabetes studies. Conducted in 23 medical centers in UK, the study investigated the glycaemia interventions of 5,102 type 2 diabetes patients. The study revealed that decreases in glycaemia level were beneficial for type-2 diabetes patients. Subsequent cost-effectiveness analysis indicated that the savings from reduction in management costs of diabetic complications were greater than the cost of glycaemia therapies (Gray \& Clarke, 2008).

The UKPDS model is a computerized simulation software developed from the

${ }^{5}$ https://www.health.govt.nz/our-work/diseases-and-conditions/diabetes/about-diabetes/virtual -diabetes-register-vdr 
UKPDS clinical trial. It can be used to estimate the life expectancy, quality adjusted life years (QALYs), and costs of diabetic complications management. The inputs of the model include age, gender, race, and duration of diabetes, height, weight, smoking status, total cholesterol, HDL cholesterol, blood pressure and glycaemia levels (HbA1c). The main outputs of the model are expected life years, QALYs, and costs of complications. Bootstrapping is used by the model to calculate the $95 \%$ confidence interval of each output (Gray \& Clarke, 2008; Clarke et al., 2004).

The diabetic complications accounted for in the model are stroke, myocardial infarction (MI), ischemic heart disease (IHD), heart failure, renal failure, amputation, and blindness. Correlations between these complications are included in the model. For instance, the probability of a patient experiencing congestive heart failure (CHF), or MI is positively correlated with systolic blood pressure, but the risk of an MI is higher for patients with a history of CHF, because of event-related dependence. A system of parametric equations is used to estimate the absolute risk of each complication, accounting for personal characteristics and medical history (Gray \& Clarke, 2008; Clarke et al., 2004). The equations used to calculate the risks of diabetic complications and deaths are listed in Table 5.12 in Appendix.

Simulations are based on annual cycles in the model. Patients begin with a given health condition (e.g., no complications) and may develop none or a few non-fatal complications or die in each cycle. Once the values for all characteristics are set, the first cycle of the model is run, in which the probability of death or of developing any of the complications is calculated. Each probability is compared with a random number drawn from a uniform distribution ranging from 0 to 1 to determine whether an event occurs or not.

In the next few sections, the inputs of the UKPDS model are discussed, and the detailed method used for estimations is explained below.

\subsubsection{Inputs of the UKPDS Model}

(1) Demographic Information

Detailed demographic information is reported in Table 5.7 below. They are from the Otago \& Ministry of Health Nutrition Survey (2017). 
Table 5.7 Demographic Information

\begin{tabular}{|c|c|c|c|}
\hline Demographic characteristics & Gender & Base Case & Sources \\
\hline \multirow[t]{4}{*}{ Age at diagnose diabetes (years) } & Male & 38 & Stats NZ \\
\hline & $(49.2 \%)$ & & \\
\hline & Female & 38 & \\
\hline & $(50.8 \%)$ & & \\
\hline \multirow{2}{*}{$\begin{array}{l}\text { Duration of diabetes at diagnose } \\
\text { (years) }\end{array}$} & male & 0 & \\
\hline & female & 0 & \\
\hline \multirow[t]{2}{*}{ Weight (kg) } & male & 85.1 & Otago \& MoH Nutrition Survey \\
\hline & female & 72.6 & \\
\hline \multirow[t]{2}{*}{ Height (cm) } & male & 175.7 & Otago \& MoH nutrition survey \\
\hline & female & 162.2 & \\
\hline \multicolumn{4}{|l|}{$\begin{array}{l}\text { Risk factor values at diagnosis of } \\
\text { type-2 diabetes }\end{array}$} \\
\hline \multirow[t]{2}{*}{ Atrial Fib. } & male & 0 & \\
\hline & female & 0 & \\
\hline \multirow[t]{2}{*}{ Smoking } & male & $17.4 \%$ & $\mathrm{MoH}$ \\
\hline & female & $14.4 \%$ & $\mathrm{MoH}$ \\
\hline \multirow[t]{2}{*}{ Chol (mmol/l) } & male & 5.09 & Otago \& MoH nutrition survey \\
\hline & female & 5.17 & \\
\hline \multirow[t]{2}{*}{ HDL (mmol/l) } & male & 1.50 & Otago \& MoH nutrition survey \\
\hline & female & 1.23 & \\
\hline \multirow[t]{2}{*}{ Sys BP (mmHg)" } & male & 130 & Otago \& MoH nutrition survey \\
\hline & female & 122 & \\
\hline \multirow[t]{2}{*}{ HbA1c (\%) } & male & 7 & Primary Care Guideline \\
\hline & female & 7 & \\
\hline
\end{tabular}

$\mathrm{MoH}=$ Ministry of Health

(2) $\mathrm{HbAlc}$

The HbA1c worksheet in the UKPDS model is used to enter HbAlc values (\%) by subject for any, or all of the years to be simulated. Figures 5.2 and 5.3 below describe the changes in glycaemia levels and the algorithm of glycaemia interventions.

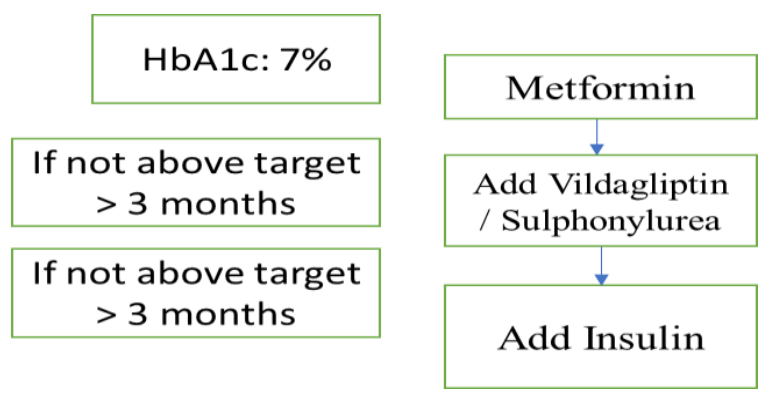

Figure 5.3 Algorithms of Diabetes Interventions 


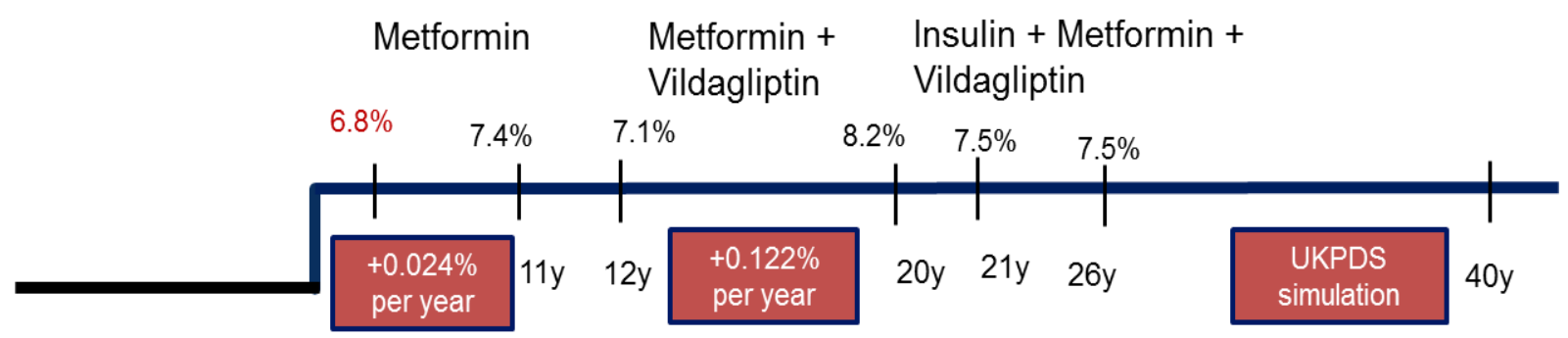

Figure 5.4 Algorithms of Diabetes Treatment \& Changes in Glycaemia Levels

As shown in the figures above, people entering the model are assumed to have no diabetic history or complications, with the glycaemia level (HbAlc (\%)) at the time of diagnosed being seven. When HbA1c is above seven, glycaemia interventions are initiated (New Zealand Guidelines Group, 2012).

Studies indicate that people using metformin monotherapy would see their HbAlc reach $6.8 \%$ at the end of the first year (Best et al., 2012). Subsequently, their HbA1c increases by approximately $0.024 \%$ per year until year 10 reaching $7.4 \%$ (UKPDS GROUP, 1998; Best et al., 2012). After that, vildagliptin should be added (New Zealand Guidelines Group, 2012). At the end of the first year of the vildagliptin plus metformin therapy, their HbA1c have decreased by $0.3 \%$ (Foley et al., 2011), and subsequently, go up by $0.122 \%$ per year until year 20 reaching $8.2 \%$ (Best et al., 2012). At this point, insulin should be added (New Zealand Guidelines Group, 2012). At the end of the first year of insulin combined with vildagliptin plus metformin treatment, their $\mathrm{HbAlc}$ would decrease to $7.5 \%$ and remain at the level in the next 5 years (Best et al., 2012).

Since there is little information on change in HbAlc after 26 years in New Zealand, HbA1c level after year 21 are predicted using the UKPDS model.

(3) Pilot Test \& Expected Lifetime

A pilot test was conducted to determine patients' expected lifetime. This information is required to decide the time horizon (the number of years simulated) in the cost analysis. In order to investigate patients' expected lifetime, both the discount rate and the disutility from diabetes and diabetic complications were set to 0 in this pilot test. The results revealed that the patients' expected lifetime was approximately 30 years. Given this, the time horizon of the diabetes cost analysis was set to be 30 years. 
(4) Costs in absence of diabetic complications

Costs in absence of diabetic complications include GP visits, glycaemia intervention costs, glycaemia test strips and needles, pharmacy service fees, and a diabetic education class in the first year. In the base case, the costs of glycaemia interventions are calculated by taking the weighted average of the costs of metformin, metformin plus vildagliptin, insulin monotherapy, and insulin combined with metformin and vildagliptin in New Zealand (Scott et al., 2014). Patients are also assumed to visit a GP three times per year and have 1.37 test strips on average per day (Scott et al., 2014). In addition, patients are assumed to attend a diabetic educational class at the year when they are diagnosed with diabetes (one-off).

In the sensitivity analysis, the costs of glycaemia interventions include only metformin monotherapy in the most conservative case. In this scenario, patients are assumed to have one glycaemia test per day and to visit a GP once per year. For the most serious condition, the costs of glycaemia interventions are changed to metformin combined with vildagliptin plus insulin, and patients are assumed to have three glycaemia tests per day and to visit a GP once per month based on experts' opinion. Details are shown in Tables 5.25 and 5.26 in Appendix.

(5) Myocardial Infarction

The cost associated with a myocardial infarction (MI) hospitalization event in the first year (non-fatal) is calculated based on the MI DRGs (DRG F10B and DRG F10A). Details are reported in Appendix.

The pharmaceutical costs associated with MI in this analysis include Cilazapril with hydrochlotothiazide, Metoprolol, statin, Marevan and Aspirin. In the base case, the dosage of Cilazapril with hydrochlotothiazide, Metoprolol, statin, Marevan, aspirin, and Clopidogrel are 5mg, $75 \mathrm{mg}, 30 \mathrm{mg}, 4 \mathrm{mg}, 100 \mathrm{mg}$, and $75 \mathrm{mg}$ per day respectively. In the sensitivity analysis, the dosage for Metoprolol, Marevan, and statin are changed to the higher and lower bound of the suggestion amount in the guideline (New Zealand Guidelines Group, 2012).

Other non-pharmaceutical costs associated with a MI include cardiology visits and 
blood tests (full blood count). In the base case, patients are assumed to have one cardiology visit and one blood test per year. In the sensitivity analysis, the frequency of cardiology visits and blood tests changes to one visit and one test per month for the most severe case. Details of the unit price, dosage, and costs for each drug in the base case and sensitivity analysis are reported in Tables 5.17 to 5.19 in Appendix.

(6) Stroke

The costs of stroke are sourced directly from Table 5.3 in this chapter. The costs of a non-fatal and a fatal stroke (died in 30 days) are approximately $\$ 30.926 .20$ and $\$ 3,470.02$ respectively in the first year, and $\$ 14.019 .08$ in subsequent years.

(7) Heart Failure

The cost associated with a heart failure hospitalization event in the first year (non-fatal) is calculated by taking the weighted average of DRG F62A and DRG62B. The cost associated with a fatal heart failure hospitalization event in the first year (fatal; died in 5 days) is based on DRG62C. Details are reported in Table 5.27 in Appendix.

The interventions for heart failure included cilazapril with hydrochlotothiazide (5mg/d), bumetanide (0.5-2mg/d), metoprolol (50-100mg/d), statin $(20-40 \mathrm{mg} / \mathrm{d})$, and aspirin (100mg/d) (New Zealand Guidelines Group, 2012). In the base case, patients are assumed to take Cilazapril with hydrochlotothiazide $(5 \mathrm{mg} / \mathrm{d})$, Bumetanide $(1 \mathrm{mg} / \mathrm{d})$, Metoprolol (75mg/d), statin (30mg/d), and Aspirin $(100 \mathrm{mg} / \mathrm{d})$ respectively. In the sensitivity analysis, the dosages for Bumetanide, Metoprolol, and statin are changed to the higher and lower bounds of the suggested amounts in the New Zealand Primary Care Handbook 2012 (New Zealand Guidelines Group, 2012). Details of the unit price, dosage, and costs for each drug are reported in Tables 5.14 to 5.16 in Appendix. Other non-pharmaceutical costs associated with heart failure include cardiology visits and blood tests (FBC). In the base case, patients are assumed to have one cardiology visit per year, and one blood test based on experts' opinion from PHARMAC. In the sensitivity analysis, the frequency is changed to one visit and one test per month for the most severe case.

(8) Kidney Failure 
The costs of kidney failure are divided into the following broad categories. They are hospitalization (in the most severe cases only), dialysis, outpatient general practitioner visits, and laboratory tests. The method used to measure each item is described below.

\section{(i) Hospitalization Costs}

The costs associated with a kidney failure hospitalization event in the first year (non-fatal) are calculated by taking the average of kidney failure DRGs (DRG L60A, DRG L60B, and DRG L60C). The costs associated with a kidney failure hospitalization event (fatal) are sourced from the item DRG L60A (See Table 5.27 for details). In the sensitivity analysis, the hospitalization costs associated with a fatal kidney failure is assumed to increase by $50 \%$ in the most severe case and decrease by $50 \%$ in the most optimistic situation.

(ii) Outpatient GP visits \& Laboratory Costs

Other costs associated with a renal failure include nephrologist visits, urine tests, and blood tests. In the base case, patients are assumed to have two nephrologist visits per year, two blood tests, and two urine tests based on experts' opinion from PHARMAC. In the sensitivity analysis, the frequency changes to four visits and four tests for the most severe case. In the most optimistic case, they are changed to one visit and one test per year based on experts' opinion.

(iii) Dialysis costs

Given that it takes a very long time for diabetes patients to progress to kidney failure, and even if they have kidney failure, it is quite unlikely to deteriorate to end stage kidney failure which requires frequent dialysis treatments, the number of dialysis treatment per week is assumed to be 2 in the base case based on experts' opinion.

Patients progressing to renal dialysis may receive hemodialysis or continuous ambulatory peritoneal dialysis (CAPD). Both treatments can be received either in the center or at home. Due to the lack of information about the proportion of self-managed dialysis patients in New Zealand, we assume that self-managed and non-self-managed dialyses are equally distributed. We therefore ultimately estimate that the expected costs of dialysis in the first year and subsequent years are $\$ 30,153.23$ and $\$ 25,827.16$ respectively, after being adjusted for inflation. Details are 
reported in Table 5.8 in Appendix.

In the sensitivity analysis, the number of dialysis treatments per week is set to three for the most severe case. In the most optimistic situation, it is changed to one treatment per week. Details are reported in Tables 5.22 to 5.24 in Appendix.

(9) IHD

The costs of IHD are sourced directly from the results reported in the section 5.3.4. The average annual costs of an IHD in the first year are $\$ 11,241.10$ (hospitalization plus treatments after discharge). The costs of IHD are approximately $\$ 1,090.10$ in subsequent years.

(10) Blindness

Green et al. (2014) found that the duration of informal care associated with blindness is approximately 14.72 hours per week. Given the median hourly wage of females in New Zealand and the fact that caregivers are more likely to be females, the costs of blindness are estimated to be approximately $\$ 18,229.14$ per year after being adjusted by the inflation rates. Other costs associated with blindness include two ophthalmology visits (only in the first year).

In the sensitivity analysis, the costs of care are increased or decreased by $50 \%$, and other costs such as costs of taxis are included in the most serious case. Literatures have shown that the main costs of blindness identified in New Zealand are probably the taxi costs (costs of informal care are not included in their study) (Godfrey \& Brunning, 2009). However, due to the small simple size and poor quality of this study, their estimation of taxi costs is considered quite unreliable. Hence these costs are only included in the sensitivity analysis. Details of the costs associated with blindness are reported in Table 5.20 in Appendix.

(11) Amputation

The cost associated with an amputation hospitalization event in the first year (non-fatal) is calculated using DRGs F11A. The cost associated with an amputation hospitalization event in the first year (fatal; died in 5 days) is based on DRG F11B (See Table 5.27 for details). In the sensitivity analysis, costs of amputation are assumed to increase or decrease by $50 \%$. 
(12) Other inputs in the UKPDS model

Given the average age of New Zealand population (38 years) and the expected life expectancy ( 82 years), the time horizon (the number of years simulated using the UKPDS model) are 40 years (maximum). Based on PFFA, the discount rate used in the model is $3.5 \%$. In addition, 1,000 loops are set in the bootstraps which are used to obtain confidence intervals.

\subsection{Disease Risks}

This section discusses the method used to measure the disease risks, $R_{0}$, and $R_{n}$. Disease risks are calculated as the ratio of cases reported over general population size. Details are discussed below.

(1) Heart disease risk

The risk of coronary heart disease is estimated based on the information listed below. According to Grey et al.'s study (2018), there were approximately 18,513 coronary heart disease (CHD) hospitalization admissions in New Zealand in 2014. Given the total New Zealand population (4,510,000 in 2014; Ministry of Health), the incidence of CHD would be approximately $41.1 / 10,000$ in 2014. Since there is a lack of information about the CHD hospitalization admissions in New Zealand after 2014, we assume that the incidence in 2019 remained the same as it was in 2014, and this value is used in the base case.

To deal with uncertainty, a sensitivity analysis is conducted in this study. In the sensitivity analysis, the number of coronary heart disease is changed to 25,460 , the number of CHD in 2006 (Grey et al., 2018). Given the New Zealand population in $2006(4,134,000$; Ministry of Health), the incidence would correspondingly change to be $62 / 10,000$.

(2) Stroke risk

According to Ranta (2015), there were 8,450 stroke hospital discharges in New Zealand in 2015. Given the total New Zealand population (4,567,000 in 2015), the incidence of stroke would be approximately 18.5 per 10,000 . Since there is little information about the number of stroke events in New Zealand after 2014, we assume that incidence in 2019 remained the same as it was in 2014. 


\section{(3) Diabetic risks}

Based on the number of people on the Virtual Diabetes Register (VDR), the proportion of $90 \%$ of all diabetes patients are type- 2 diabetes patients in New Zealand (Ministry of Health, 2019). Given the number of type-2 diabetes patients and the total New Zealand population in each year (Ministry of Health), the incidence of diabetes for the year 2013 to 2018 is calculated. In the base case, the average of these is used in the analysis for the incidence of diabetes in 2019.

In the sensitivity analysis, two other values for incidence in 2019 are considered. (1) The figure for 2018. (2) The value estimated from applying the projective forecasting method. In this method, a linear regression is conducted and further used to predict the value. Details of the calculations are shown in Figure 5.8 in Appendix.

(4) Convert the yearly risk to the ten-year risk

Yearly incidence is then converted to a ten-year rate following the decreasing exponential approximation of life expectancy (DEALE) method (Beck, Kassirer and Pauker, 1982). Details of the calculation are shown in Appendix IV.

\subsection{SSB Consumption}

Data on SSB consumption is sourced from the estimation discussed in chapter 4 . In the base case, SSB consumption per person per year is 70.71 litres. In the sensitivity analysis, the SSB consumption per person per year is 68.36 litres.

\subsection{Results}

\subsubsection{Lifetime Stroke Costs}

The probability of developing a stroke for a light drinker is approximately 0.0183 over 10 years. Given the hazard ratio between light drinkers and non-drinkers (1.07), the probability of developing a stroke for a non-drinker is $0.0183 / 1.07=0.0171$ over 10 years. Therefore, light drinkers are expected to cost $\$ 87,166.35 *(0.0183-0.0171)=$ $\$ 104.60$ more than non-drinkers. Given the SSB consumption per person per year $(70.71 \mathrm{~L})$, the average cost per litre is estimated to be $\$ 104.60 /(70.71 * 10)=\$ 0.148$.

Sensitivity analysis results are reported in Table 5.28 below. It shows that the result is quite sensitive to the severity of a stroke. Detailed calculation is reported in Appendix. To sum up, the point estimation of the harm of a stroke from SSB consumption is 
$\$ 0.148$ per litre, with a possible range of $\$ 0.009$ to $\$ 0.377$ per litre.

Table 5.28: Sensitivity Analysis Results of Lifetime Costs \& Harm/Litre Associated with Stroke

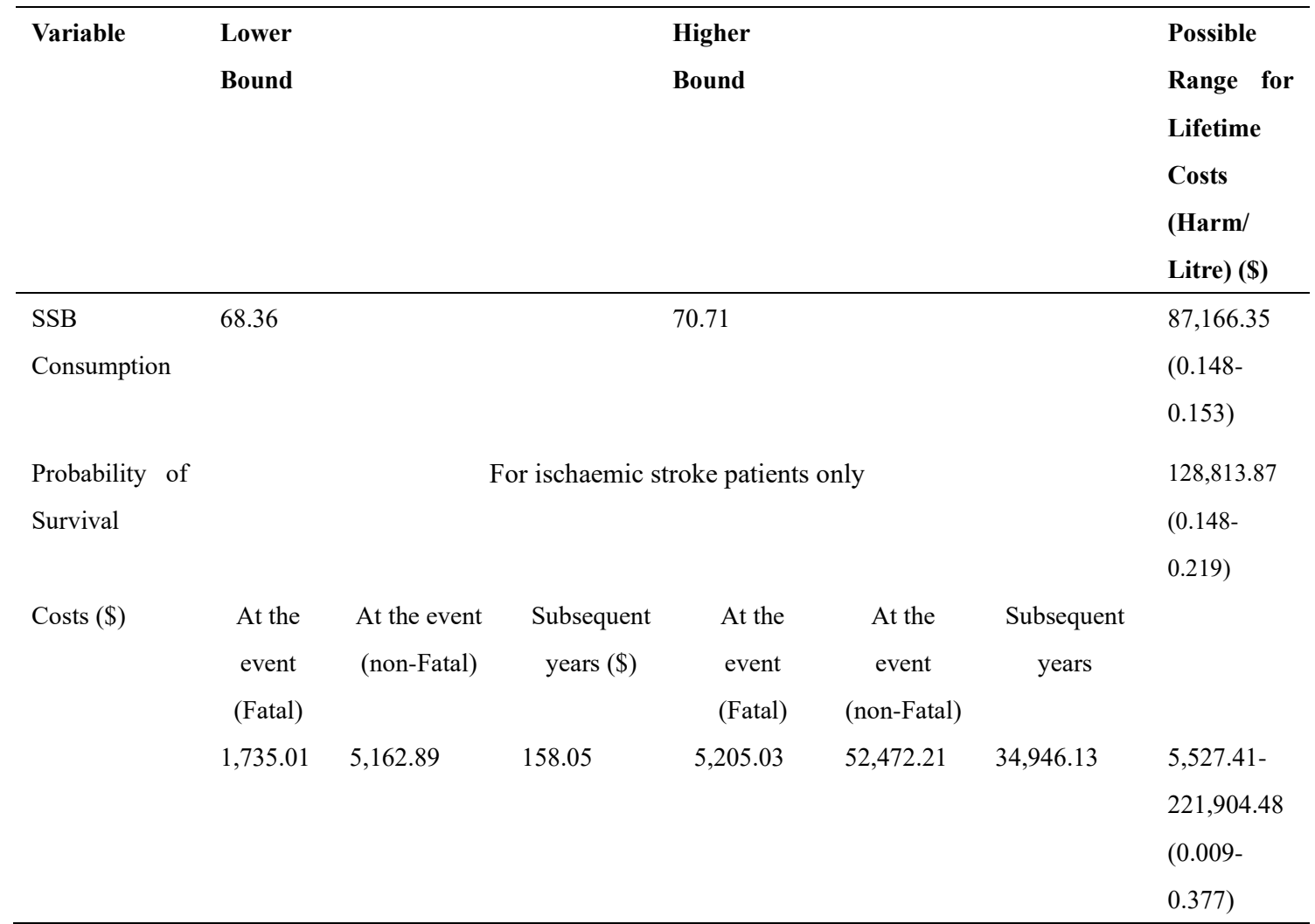

\subsubsection{Lifetime IHD Costs}

The costs of IHD are approximately $\$ 11,241.10$ in the first year, and $\$ 1,090.10$ in subsequent years, respectively. Given the proportion of people who are alive in each year, the lifetime costs after discounting are approximately $\$ 17,018.60$.

The probability of developing an IHD for a light drinker is approximately 0.040 in 10 years. Given the hazard ratio between light drinkers and non-drinkers (1.02), the probability of developing an IHD for a non-drinker is $0.0403 / 1.02=0.0396$ in 10 years. Thus, the light-drinkers are expected to cost $\$ 17,018.60 *(0.0403-0.0396)$ $=\$ 11.91$ more than non-drinkers. Given SSB consumption per person per year (70.71L), the average cost per litre is estimated to be approximately $\$ 11.91 /$ $(70.71 * 10)=\$ 0.017$.

Sensitivity analysis results are reported in Table 5.29 below. The result is quite sensitive to the value of incidence used in the estimation and the severity of an IHD. Overall, the point estimation of the harm of IHD from SSB consumption is $\$ 0.017$ per litre, with a possible range of $\$ 0.009$ to $\$ 0.029$ per litre. Detailed calculation is 
reported in Appendix.

Table 5.29 Sensitivity Analysis: Lifetime Costs \& Harm/Litre Associated with IHD

\begin{tabular}{|c|c|c|c|c|c|}
\hline Variable & & bound & & r bound & $\begin{array}{l}\text { Possible Range for } \\
\text { Lifetime Costs } \\
\text { (Harm/Litre) (\$) }\end{array}$ \\
\hline $\begin{array}{l}\text { SSB } \\
\text { Consumption }\end{array}$ & & 68.36 & \multicolumn{2}{|c|}{70.71} & $\begin{array}{l}\$ 17,018.60 \\
(0.017-0.017)\end{array}$ \\
\hline Incidence & \multicolumn{2}{|c|}{0.0028} & \multicolumn{2}{|c|}{0.0062} & $\begin{array}{l}\$ 17,018.60 \\
(\$ 0.012-0.029)\end{array}$ \\
\hline $\begin{array}{l}\text { Proportion } \\
\text { of People are } \\
\text { Survival }\end{array}$ & \multicolumn{4}{|c|}{ from the data of the patients in the Invercargill hospital } & $\begin{array}{l}\$ 15,957.03-\$ 17,018.60 \\
(0.016-0.017)\end{array}$ \\
\hline \multirow[t]{2}{*}{ Costs } & At the event & $\begin{array}{l}\text { Subsequent } \\
\text { years }\end{array}$ & At the event & $\begin{array}{l}\text { Subsequent } \\
\text { years }\end{array}$ & \\
\hline & $\$ 7,494.07$ & $\$ 425.10$ & $\$ 16,861.65$ & $\$ 1,484.11$ & $\begin{array}{l}\$ 8,888.54-\$ 24,288.10 \\
(\$ 0.009-\$ 0.024)\end{array}$ \\
\hline
\end{tabular}

\subsubsection{Lifetime Diabetes Costs}

The costs of diabetes treatments in absence of complications are $\$ 941.91$ per year in the base case. The costs of IHD, MI, heart failure, stroke, renal failure, blindness, and amputation are summarized in Table 5.10 in Appendix. Given these costs, the lifetime total costs of diabetes are calculated by the UKPDS model. As shown in Table 5.9 below, the total costs of diabetes after discounting are $\$ 30,202.59$ cross 40 years, and life expectancy are 18.7 years.

Table 5.9: UKPDS Results: Diabetes Total Costs \& Life Expectancy (Base Case)

\begin{tabular}{lccc}
\hline Outputs & Point Estimation & \multicolumn{2}{c}{$\mathbf{9 5 \%}$ Confidence Interval } \\
& & Lower & Upper \\
\hline Total costs (\$) & $30,202.59$ & $24,767.89$ & $35,637.29$ \\
Life expectancy after discounting (years) & 18.7 & 17.8 & 19.7 \\
\hline
\end{tabular}

The probability of developing diabetes for a light drinker is 0.01616 in 10 years. Given the hazard ratio between light drinkers and non-drinkers (1.07), the probability of developing diabetes for a non-drinker is $0.01616 / 1.07=0.01510$ in 10 years. Therefore, the light-drinkers are expected to cost $\$ 30,202.59 *(0.01616-0.01510)=$ \$32.02 more, compared with non-drinkers. Given the SSB consumption per person per year $(93.38 \mathrm{~L})$, the average cost per litre is $\$ 32.02 /(70.71 * 10)=\$ 0.045$.

Sensitivity analysis results are reported in Table 5.30 below. Detailed calculation is 
reported in Appendix. The result has been shown quite sensitive to the risk of diabetes for an ordinary people and the costs in absence of diabetic complications.

Above all, the point estimation of the diabetic harm from SSB consumption is $\$ 0.045$ per litre, with a possible range $\$ 0.023$ to $\$ 0.082$.

Table 5.30: Sensitivity Analysis of Costs Associated with Diabetes

\begin{tabular}{llll}
\hline Variable & Lower bound & Higher Bound & Possible Range (\$/Litre) \\
\hline Diabetes Incidence in 2019 & 0.0008 & 0.01616 & $0.023-0.045$ \\
SSB consumption & 68.36 & 70.71 & $0.045-0.047$ \\
$\begin{array}{l}\text { Costs in Absence of Diabetic } \\
\text { Complications }\end{array}$ & 168.7 & $2,423.11$ & $0.026-0.082$ \\
\hline
\end{tabular}

\subsubsection{Total Harm from Externalities}

Given the harm of IHD from SSB consumption ( $\$ 0.017$ per litre), the harm of a stroke (\$0.148 per litre), and the harm of diabetes ( $\$ 0.045$ per litre), the marginal harm associated with externalities can be calculated as the summation of the three, which is $\$ 0.21$ per litre.

\subsection{Discussion}

The cost of stroke and IHD are calculated as a proportion of diabetes lifetime cost in the UKPDS model. Such costs would be double counted if the increase in IHD or stroke risk is purely a result of diabetes progression. In order to address this concern, the diabetes patients' risk of developing an IHD or stroke is compared with general population's (they are assumed to be SSB drinkers) risks. As shown in Figure 5.9 in Appendix, the incidence of developing an IHD in a year predicted by the UKPDS model ranges from 0.002 to 0.005 for diabetes patients. As discussed in section 5.4 in this chapter, the incidence of IHD for a light SSB drinker and a non-drinker is 0.0041 and 0.0040 respectively, with a possible range of 0.0028 to 0.0062 for a light SSB drinker and 0.0027 to 0.0061 for a non-drinker. Since that diabetic patients' risk of developing an IHD is quite close to the risk for general population, it seems that the increase in IHD risks is not a result of progression of diabetes, and therefore the costs of an IHD are not double counted in this study.

Similarly, as shown in Figure 5.8, the incidence of developing a stroke in a year predicted by the UKPDS model ranges from 0.0004 to 0.002 for diabetes patients. As 
discussed in section 5.4 in this chapter, the incidence of stroke for a light SSB drinker and a non-drinker is 0.00185 and 0.00173 , respectively. Since diabetic patients' risk of developing a stroke is quite close to the risk of general population, it seems that the increase in stroke risks are not a result of progression of diabetes, and hence costs of a stroke are not double counted in this study.

There are a few limitations in this study. Firstly, calculations are conducted necessarily based on many assumptions, and it is not very clear how far some of the assumptions deviate from real situations in New Zealand. For example, there is little updated information about IHD or stroke incidences after 2014, so the value of the incidence used in the analysis has a high degree of uncertainty associated with it. Therefore, it would be valuable to update the results with new information when it becomes available in the future.

Secondly, the diabetes incidence used in the analysis is considered to have high uncertainty. As shown in Figure 5.8 in Appendix, the incidence rate per year had been declining from 2011 to 2017 , followed by a sudden increase in the year 2018, which makes the prediction quite challenging. As discussed previously, in order to address this problem, the results are tested by varying the incidence rate over a wide range of possible values. Although result has been seen quite sensitive to the diabetes incidence, the true value is thought highly likely in the possible range reported in the sensitivity analysis.

Third, the annual incidences in this study are calculated based on hospital admissions only, so mild cases which do not require hospital admissions are not included. As a result, the incidences estimated in this study are very likely to be underestimated. Similarly, renal failure patients who are on dialysis treatment for a short duration before having a kidney transplant are not included in the analysis. This concern is somewhat moderated by the findings of that the total costs of diabetes and diabetic complications have been shown not sensitive to the costs of renal failure at all.

Fourth, the annual probability of survival after an IHD is derived from the Kaplan-Meier survival curves for IHD patients in Dunedin Hospital and Invercargill Hospital. Given that there is a bigger elderly population in the Otago and the Southern 
District than in other places in New Zealand, whether they are good representatives of an average New Zealand IHD patient population could be questioned.

In addition, we did not account for the complementary effects between treatments for stroke and heart diseases (only complementary effects between stroke and heart disease for diabetes patient are modeled in the UKPDS model). For example, a heart disease patient may benefit from lowering the chance of developing a stroke as a result of heart disease treatments. However, this concern is somewhat moderated by the fact that externalities are quite negligible.

Furthermore, the exponential function used in the regression do not fit the survival curve (Figure 5.4) very well, and the $\mathrm{R}^{2}$ is only 0.76 . However, we think it may not be a serious concern, because the difference between the values estimated from the exponential function and the value in reality is considered very tiny after discounting, so the impact on results is highly likely in the possible range estimated in the sensitivity analysis.

The pharmacy margin for a pharmaceutical with a list price per pack under $\$ 150$ is 3\% per pack in New Zealand, and each pharmacy dispensing incurs a cost of $\$ 5.44$ as a pharmacy handling and service fee $(\mathrm{MoH}, 2019)$. These costs are not calculated explicitly in this study, partly because there is little information about the frequency of stroke, IHD, and diabetes patients going to a pharmacy to fulfill a prescription. In addition, given that the uncertainty associated with the pharmacy margin and dispensing costs is probably very small, it is thought not to have a substantial effect on the results.

Furthermore, there are confidential rebates of some of the drugs used in the treatments of a stroke or an IHD or diabetes (the PHARMAC online schedule shows the gross price, rather than the net price after rebates). As a result, the costs in subsequent years are considered with some uncertainty. Also, some drug costs may have been double counted: if patients experienced both a stroke and an IHD, a statin can be used for both after a stroke or a MI. In order to allow for the uncertainty, costs of an IHD or a stroke are varied by $50 \%$. This is considered reasonable and necessary.

The costs of heart disease and renal failure are considered to change soon in New 
Zealand, because GLP-1 receptor agonists and sodium-glucose transport protein two (SGLT-2) inhibitors are probably going to be funded by PHARMAC for diabetes patients with renal failure and heart disease respectively. Therefore, the costs of treatments in the future are probably going to increase. Fortunately, as the total costs have been found not sensitive to the costs of renal failures and heart diseases in the sensitivity analysis, the increase in costs due to the funding of GLP-1 and SGLT-2 is considered unlikely to have substantial effects.

In addition, the parameters used in the UKPDS model, such as transitional probabilities, are from the UKPDS study. These values are calculated from the type-2 diabetes patients in the 23 medical centers in UK in which patients in the trail are Europeans, Africans, and Indians. Apparently, the race distribution of in the trial is different from New Zealand population. As a result, whether the parameters in the model can be applied to New Zealand population should be questioned.

Finally, in the clinical reports, the decrease in HbAlc is always associated with a decrease in people's weight. However, the UKPDS model only accounts for the weights at base line; and there is no input spreadsheet in the UKPDS model where the change in weight can be entered as inputs together with the HbAlc levels. As a result, it is not sure how the results are affected by this weakness.

Despite of the limitations mentioned above, our findings in the study are considered sound and quite reliable, because our estimates are quite close to the values in the NZIER report. Given the costs of a non-fatal and a fatal stroke in the first year, the costs of a non-fatal stroke in subsequent years, the proportion of people who are alive in each year, and the $3.5 \%$ discount rate, the expected costs in the first 5 years are estimated to be approximately $\$ 52,292.14$, after being adjusted for inflation. This is very close to the findings in the NZIER report in which their expected costs in the first 5 years are \$54,234.27 (NZIER, 2018).

\subsection{Conclusion}

In this chapter, we estimate the harm of SSBs associated with externalities by assessing the lifetime cost of diabetes, stroke, and IHD. To sum up, the point estimation of the harm of a stroke from SSB consumption is $\$ 0.148$ per litre, with a 
possible range of $\$ 0.009$ to $\$ 0.377$ per litre; the point estimation of the harm of IHD from SSB consumption is $\$ 0.017$ per litre, with a possible range of $\$ 0.009$ to $\$ 0.029$ per litre; and the point estimation of the diabetic harm from SSB consumption is $\$ 0.045$ per litre, with a possible range $\$ 0.023$ to $\$ 0.082$. Finally, the marginal harm associated with externalities is estimated to be $\$ 0.21$ per litre. 


\section{A Simulation Using Real World Data}

\subsection{Introduction}

In this chapter, we calibrate the model constructed in chapter three to estimate the optimal tax rate and investigate the question of how this rate changes as complements and substitutes are accounted for. In addition, the welfare improvement generated by taxes is also simulated with real world data. The result can inform the question of which tax base is best, and whether general beverage taxes are superior to SSB taxes. The primary calibration uses New Zealand data from Ni Mhurchu et al. (2012). This chapter also reports calibration exercises using Australian information from Sharma et al. (2014) and American data from Dharmasena and Capp's (2011) respectively.

Recall that in chapter 3, there are $\mathrm{N}$ different types of drink, $\mathrm{K}$ of which are SSBs. The harmful ingredient per litre of drink $\mathrm{j}$ is denoted by $\mathrm{r}_{\mathrm{j}}$. The harm per calorie to the drinker is $\mathrm{H}_{\mathrm{I}}, \beta \in(0,1)$ represents the proportion of the health harm taken into account by consumers, harm from externalities is $\mathrm{H}_{\mathrm{E}}$, and $\mathrm{b}_{\mathrm{j}}$ is the tax base for drink j. For a tax by litres, $b_{j}=1$; for an ad valorem tax, $b_{j}=c_{j}$; and for a tax by the harmful ingredient contained $b_{j}=r_{j}$.

The optimal tax rate is derived using a utility-maximization model, accounting for complements and substitutes. The optimal tax levied by the government $\tau$ is:

$$
\tau=\left[(1-\beta) H_{I}+H_{E}\right] \frac{\sum_{j=1}^{N} r_{j} \frac{d q_{j}}{d \tau}}{\sum_{j=1}^{K} b_{j} \frac{d q_{j}}{d \tau}} .
$$

The optimal tax rate is composed of two terms: (1) the proportion of harm to the consumer that is not reflected in consumption decisions, (2) a scaler for the tax which reflects tax impacts. Recall that in section 3.3, the scaling factor was phrased in terms of elasticities, quantities, and prices. The result is: 


$$
\frac{\sum_{j=1}^{N} r_{j} \frac{d q_{j}}{d \tau}}{\sum_{j=1}^{K} b_{j} \frac{d q_{j}}{d \tau}}=\frac{\sum_{j=1}^{N}\left(r_{j} \sum_{i=1}^{K} \varepsilon_{i j} \frac{q_{j}}{p_{i}} b_{i}\right)}{\sum_{j=1}^{K}\left(b_{j} \sum_{i=1}^{K} \varepsilon_{i j} \frac{q_{j}}{p_{i}} b_{i}\right)} .
$$

Moreover, in chapter 3, a welfare measurement is developed to evaluate the change due to SSB taxes, which is:

$$
\Delta \mathrm{W}=\mathrm{U}\left(\mathrm{q}_{1}\right)-\mathrm{U}\left(\mathrm{q}_{0}\right)+\tau \mathrm{q}_{1}+\left(\mathrm{H}_{\mathrm{I}}+\mathrm{H}_{\mathrm{E}}\right) \mathrm{r}^{\mathrm{T}}\left(\mathrm{q}_{0}-\mathrm{q}_{1}\right),
$$

and $\mathrm{U}(\mathrm{q})$ is:

$$
U(q)=m^{T} q-0.5 q^{T} n q-p q
$$

where $m$ and $n$ are:

$$
\begin{gathered}
\mathrm{m}=-\left\{0.5 *\left[\mathrm{~B}+\mathrm{B}^{\mathrm{T}}\right]\right\}^{-1}\left\{\mathrm{q}_{0}-0.5 *\left[\mathrm{~B}+\mathrm{B}^{\mathrm{T}}\right] \mathrm{p}_{0}\right\} \\
\mathrm{n}=-\left\{0.5 *\left[\mathrm{~B}+\mathrm{B}^{\mathrm{T}}\right]\right\}^{-1}
\end{gathered}
$$

The price and the quantity of drinks are $\mathrm{p}$ and $\mathrm{q}$ respectively. The initial (observed) prices and quantities are $\mathrm{p}_{0}$ and $\mathrm{q}_{0}$; the post-tax prices and quantities are $\mathrm{p}_{1}$ and $\mathrm{q}_{1}$. The harm reductions due to taxes are $\left(H_{I}+H_{E}\right) r^{T}\left(q_{0}-q_{1}\right)$. Finally, matrix $B$ is an array of the slopes of the demand curves.

$\mathrm{U}\left(\mathrm{q}_{1}\right)-\mathrm{U}\left(\mathrm{q}_{0}\right)$ is the decrease in utility due to reduction in beverage consumption. Tax revenue is $\tau q_{1}$, so that $\mathrm{U}\left(\mathrm{q}_{1}\right)-\mathrm{U}\left(\mathrm{q}_{0}\right)+\tau \mathrm{q}_{1}$ is the deadweight loss (DWL) due to taxes. This approach is equivalent to using a multi-commodity DWL measure, with linear approximations of demand curves. The setup is relatively simple but robust and is suitable for calibrations that use estimates which are derived from a range of different approaches.

\subsection{Method \& Data Sources}

\section{(1) Elasticities, Prices, Quantities \& Energy Contents}

The primary simulation is calibrated with data on elasticities, prices, and quantities of carbonated soft drinks, energy drinks, and other non-alcoholic beverages in New Zealand in 2009/10 (Ni Mhurchu et al., 2013). Data on sugar content is reported by Smith et al. (2010).

As the categories of beverages in Ni Mhurchu et al. and Smith et al. are different, the following assumptions and modifications are made to make the categories consistent. First, cola, diet cola, and ginger ale are categorized as carbonated soft drinks. They constitute approximately $26.2 \%, 47.6 \%$, and $26.2 \%$ of all soft drink consumption 
respectively (Sharma et al., 2014). Given the proportions of each drink consumed and the calories each beverage contains, the weighted average energy content (per 8 ounces) of carbonated soft drink is approximately 47.97 calories. Second, other non-alcoholic beverages include apple juice, orange juice, tea and coffee, as well as bottled water. They constitute $4.6 \%, 4.6 \%, 88.5 \%$, and $2.2 \%$ of all non-alcoholic beverage consumption respectively (Ni Mhurchu et al., 2013). Based on this, the weighted average energy per 8 ounces of non-alcoholic beverages is approximately 12.2 calories.

\section{(2) Harm from Internalities}

(a) Harm per Litre

According to the findings reported in chapter 4, if we assume that the representative consumer consumes the average amount of SSBs, then he or she is classified as a light-to-medium drinker (Malik, 2017) whose decisions only take into account marginal harm $\left(\beta H_{I}\right)$ of $\$ 15.52$ according to the preliminary analysis. Given marginal harm taken into account of $\beta H_{I}=\$ 15.52$, and a value of $\beta$ of 0.6 (Paserman, 2008), we can back out the marginal harm to the consumer as $\mathrm{H}_{\mathrm{I}}=25.87$ per litre. Then the marginal harm that is not taken into account in consumption decisions is $(1-\beta) H_{I}=$ $\$ 10.35$ per litre.

(b) Harm per Calorie

Under the assumption that the total harm of consuming soft drinks is proportional to the total amount of harmful ingredients, it is convenient and necessary to convert harm per litre to harm per calorie. Given that SSB consumption is 45 gallons per person per year (Andreyeva et al. 2009) and that daily energy intake from SSBs is around 156 calories per day (Smith et al., 2010), average energy per litre would be approximately 334.26 calories. The portion of marginal harm that is not considered ( $\$ 10.35$ per litre) would be equivalent to $\$ 0.0310$ per calorie.

\section{(3) Harm from Externalities}

As argued in Chapter 5, given people's WTP for health risk reductions, litres of SSBs consumed per person on average, and the increased health risk associated with SSB consumption, the marginal harm associated with externalities is estimated to be $\$ 0.21$ 
per litre, which is equivalent to $\$ 0.0006$ per calorie.

\section{(4) Total Harm}

The total harm can be calculated as a summation of internalities and externalities. They are $\$ 10.56$ per litre (around $\$ 6.34$ for a bottle of $600 \mathrm{ml}$, $\$ 3.70$ for a can of $350 \mathrm{ml})$, and equivalent to $\$ 0.032$ per calorie.

\section{(5) Sensitivity analysis}

One-way and two-way deterministic sensitivity analyses are applied to evaluate the robustness of the results based on the following possible values for each parameter. (1) The marginal harm is changed to $\$ 0.0006$ per calorie. In this situation, only the harm associated with externalities is addressed, because internalities are probably more controversial to be intervened compared with externalities (2) The marginal harm is changed to $\$ 0.0006$ per calorie, and the elasticities, prices, and consumptions are changed to the values reported by Sharma et al. (2014), and by Dharmasena and Capp's (2011) respectively.

\subsection{Results}

\section{Table 6.1 Results of Simulation}

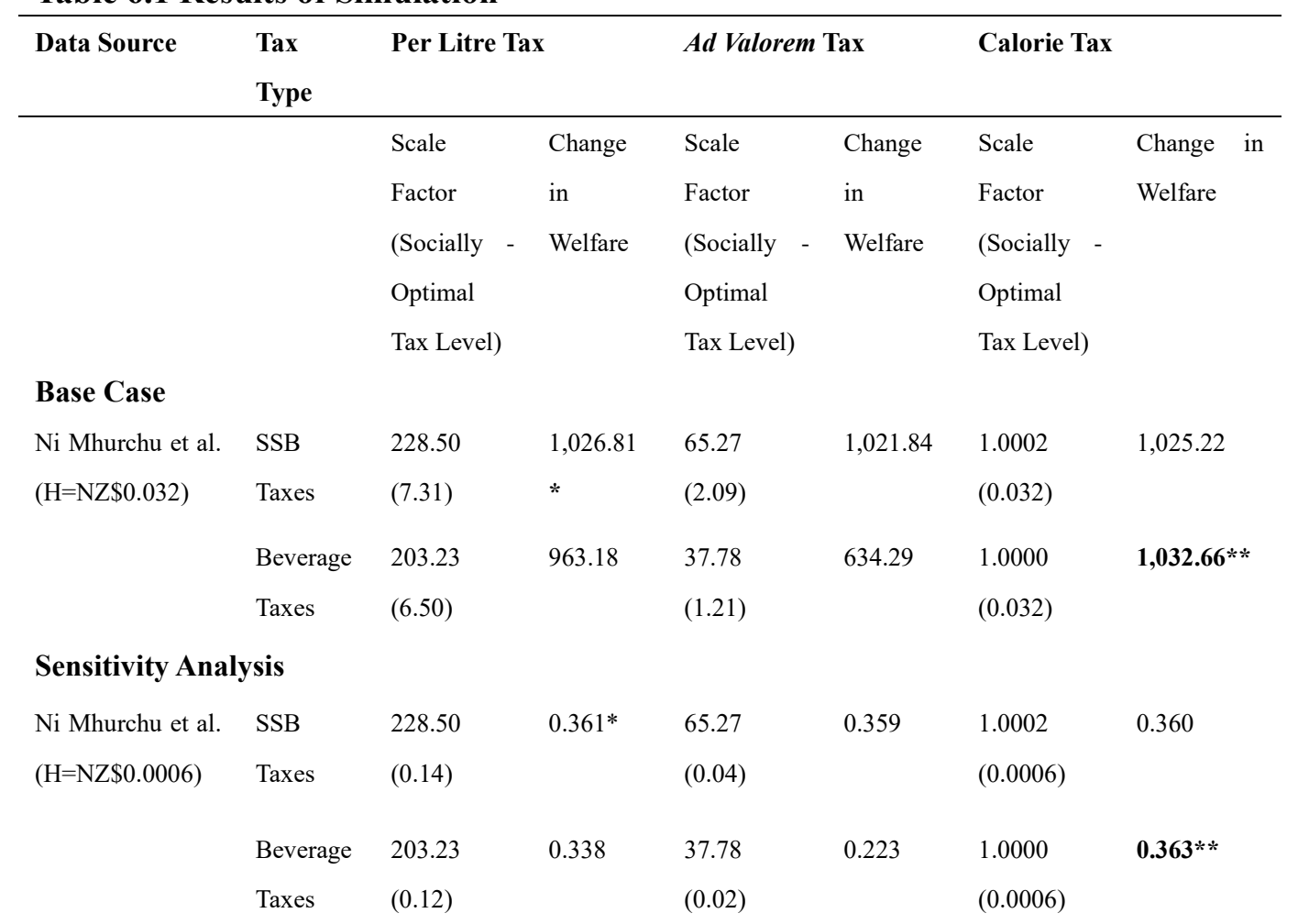




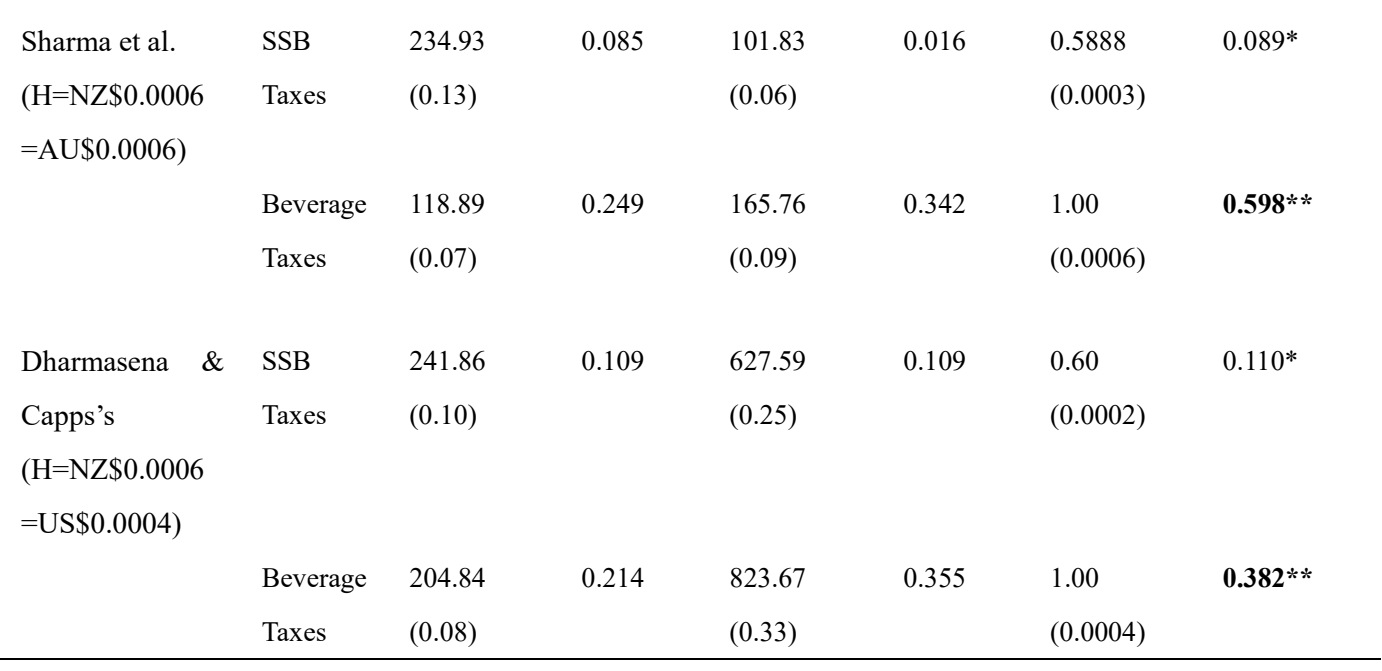

* the highest increase in welfare in a certain category of tax

** the highest increase in welfare in a certain category of all taxes

\subsubsection{Base Case}

(i) Socially Optimal Level

As shown in Table 6.1 above, the elasticity estimates from Ni Mhurchu et al. imply that when marginal harm is $\$ 0.032$ per calorie, the socially optimal levels for a per litre SSB tax, an ad-valorem SSB tax, and a SSB tax by calorie are 7.31 (around \$4.39 for a bottle of $600 \mathrm{ml}$ ), 2.09 (price increased by 209\%), and 0.032 (around $\$ 6.42$ for a bottle of $600 \mathrm{ml}$ ) respectively. The socially optimal levels for a per litre beverage tax, an ad-valorem beverage tax, and a beverage tax by calorie are 6.50 (around $\$ 3.90$ for a bottle of $600 \mathrm{ml}$ ), 1.21 (price increased by 121\%), and 0.032 (around $\$ 6.42$ for a bottle of $600 \mathrm{ml}$ ) respectively.

(ii) Tax Base \& Welfare

In the base case, all the taxes considered would increase welfare from the original point where no tax is imposed. A beverage tax by calorie is the most favorable, and a beverage tax by price is the least favorable. The intuition is that calories are the harmful ingredients, so tax by calorie has the strongest correlation with the harmful ingredient, while price has the weakest correlation with it. If a tax per calorie is set and applied to all drinks, it will correct all distortions, yielding the highest attainable welfare. In order to verify this argument, the correlations between tax bases and harmful ingredients are calculated, and reported in Table 6.2 in Appendix V. The calculation reveals that the correlation between calories and the harmful ingredients is 
1 , and correlation between prices and harmful ingredient is -0.699 , which is the lowest value in all correlations. The results are consistent with our intuition.

(iii) Multiplier

Recall that the expression for the optimal tax rate involves a multiplier applied to harm not taken into account. The multiplier reflects the pattern of substitutes and complements. Because the multipliers (a SSB tax by calories and a beverage tax by calories) are greater than one, so the tax rate becomes larger when substitutes and complements are considered. This is probably because (1) substitute effects are quite weak (cross price elasticities are close to 0 ), and (2) substitutes contain lower levels of harmful ingredients. These factors suggest a heavier tax.

\subsubsection{Sensitivity Analysis}

Next, the sensitivity of the preceding results is examined. First, consider how the optimal tax rate would be revised if internalities were not considered legitimate concern for policy. When marginal harm is $\$ 0.0006$ per calorie (as it would be if only externalities were addressed), the socially optimal levels for a per litre SSB tax, an ad-valorem SSB tax, and a SSB tax by calorie are 0.14 (around $\$ 0.08$ for a bottle of $600 \mathrm{ml}$ ), 0.04 (price increased by 4\%), and 0.0006 (around $\$ 0.12$ for a bottle of $600 \mathrm{ml}$ ) respectively in New Zealand. The socially optimal levels for a per litre beverage tax, an ad-valorem beverage tax, and a beverage tax by calorie are 0.12 (around $\$ 0.07$ for a bottle of $600 \mathrm{ml}$ ), 0.02 (price increased by 2\%), and 0.0006 (around $\$ 0.12$ for a bottle of $600 \mathrm{ml}$ ) respectively.

As in the base case, all the taxes increase welfare from the original point where no tax is imposed. A beverage tax by calorie continues to be the most favorable tax base, and a beverage tax by price is the least favorable. Finally, as the multiplier of a per calorie SSB tax is greater than 1, the tax rate will become larger if substitutes and complements are considered. These findings remain consistent with base case.

In the second sensitivity exercise, elasticity estimate from Ni Mhurchu et al. (2013) are replaced with those reported by Sharma et al. (2014), and only externalities are considered legitimate to be addressed. The results imply that when marginal harm is NZ\$0.0006 per calorie, the socially optimal levels for a per litre SSB tax, an 
ad-valorem SSB tax, and a SSB tax by calorie are 0.13 (around $\$ 0.08$ for a bottle of $600 \mathrm{ml}$ ), 0.06 (price increased by $6 \%$ ), and 0.0003 (around $\$ 0.06$ for a bottle of $600 \mathrm{ml}$ ) respectively. The socially optimal levels for a per litre beverage tax, an ad-valorem beverage tax, and a beverage tax by calorie are 0.07 (around $\$ 0.04$ for a bottle of $600 \mathrm{ml}$ ), 0.09 (price increased by $9 \%$ ), and 0.0006 (around $\$ 0.12$ for a bottle of $600 \mathrm{ml}$ ) respectively. A beverage tax by calories is still the most favorable, but an ad valorem SSB tax now is the least favorable tax base. The causes of the change will be discussed in the discussion section of this chapter. In addition, in contrast to the base case, as the multiplier of per calorie SSB tax is smaller than 1, the tax rate becomes smaller when substitutes and complements are taken into account. This is because the reported substitute effects are quite strong in Australia, and the substitutes contain higher amounts of harmful ingredients. These factors suggest the tax rate should not be moderated. Otherwise, consumers would switch to those untaxed high calorie foods and yield a worse outcome. In extreme cases, substitution to higher calorie beverages can imply that a SSB tax could be harmful rather than beneficial to society. In the third sensitivity exercise, elasticity estimate from Ni Mhurchu et al. (2013) are replaced with those reported by Dharmasena and Capps (2012), and only externalities are considered legitimate to be addressed. The results imply that when marginal harm is NZ\$0.0006 (equivalent to US\$0.0004) per calorie, the socially optimal levels for a per litre SSB tax, an ad-valorem SSB tax, and a SSB tax by calorie are 0.10 (around US\$0.06 for a bottle of $600 \mathrm{ml}$ ), 0.25 (price increased by 25\%), and 0.0002 (around US\$0.04 for a bottle of $600 \mathrm{ml}$ ) respectively. The socially optimal levels for a per litre beverage tax, an ad-valorem beverage tax, and a beverage tax by calorie are 0.08 (around US\$0.05 for a bottle of $600 \mathrm{ml}$ ), 0.33 (price increase by 33\%), and 0.0004 (around US\$0.08 for a bottle of $600 \mathrm{ml}$ ) respectively. A beverage tax by calories is still the most favorable, but an ad valorem SSB tax now is almost as ineffective as a volumetric tax. As with the scenario using Sharma et al.'s study, because of strong substitution effects between SSBs and fruit juice that contains high amounts of calories, the optimal tax rate will become smaller when substitutes and complements are taken into account. This suggests that a high tax rate is not encouraged in this 
situation.

\subsection{Discussion}

(1) Socially - Optimal Level

As shown in Table 6.1, the calculated socially optimal levels are much higher than most SSB taxes imposed in the real world (Falbe et al., 2015; Fletcher, Frisvold \& Tefft, 2014; Silver et al., 2017; Cawley et al., 2019). In the base case, when both externalities and internalities are addressed, the optimal level of volumetric taxes will be approximately NZ\$6 to NZ\$7 per litre, and the optimal level of ad valorem taxes is around $120 \%$ to $210 \%$ under the $100 \%$ pass-through assumption. If the pass-through rate was below $100 \%$, the optimal tax rate would be even higher. This situation is reasonably plausible as studies have estimated that pass-through rates vary from around $50 \%$ to $100 \%$ (Falbe et al.; 2015; Colantuoni, 2014). However, real world volumetric taxes vary from $\mathrm{NZ} \$ 0.1$ to $\mathrm{NZ} \$ 0.8$ per litre (after being adjusted by the exchange rate); and ad valorem taxes in real world are approximately $10 \%$ to $20 \%$. Exceptions are Bahrain and Saudi Arab. They impose a 100\% excise energy drink tax (equivalent to $100 \%$ ad valorem taxes)) (Cawley et al., 2019). The tax rates in the real world would be reasonably close to the estimated optimal tax levels if only externalities were accounted for. This is probably because it is more controversial to intervene to address internalities than externalities.

(2) Are Beverage Taxes Better Than SSB Taxes?

The findings of the previous section 6.3 suggest that a beverage tax by calories is the most favorable option, as it has a perfect relationship with harmful ingredients. This conclusion is consistent with the findings of Grummon et al. (2019) and Zhen et al. (2014). However, the ranking of ad valorem beverages taxes, volumetric beverage taxes, and SSB taxes may depend on specific circumstances. Details are discussed below.

In Ni Mhurchu et al.'s study, the substitution effects are quite weak (many cross-price elasticities of the taxed SSBs are close to zero) (Ni Mhurchu et al., 2013). Therefore, taxing SSBs does not substantially increase the consumption of untaxed products. In addition, we note that those untaxed non-alcoholic beverages have lower harmful 
ingredients compared with SSBs. Consequently, it would be reasonable to expect that if SSB taxes are imposed, the harm reduction will be considerable. Moreover, the optimal SSB tax rates are much higher than the optimal beverage tax rates estimated from the data reported by Ni Mhurchu (2013). All these factors lead to a result that the harm reduction from SSB taxes is greater than that from beverage taxes (except for beverage taxes by calorie).

Compared with the situation when only SSBs are taxed, non-alcoholic low calorie beverages such as unsweetened tea and coffee would be covered by a tax on all beverages. As a result, the loss of consumers' enjoyment will become much larger in this situation, but the marginal health benefits of such taxes are relatively small (except for when beverage taxes by calorie are imposed).

Given that SSB taxes generate more health benefits and cause a relatively small utility loss, SSB taxes ultimately yield higher total welfare than ad valorem beverage taxes and volumetric beverage taxes. In order to check these arguments, the changes in utility and the harm reduced by taxes are reported in Tables 6.2 and 6.3 in Appendix V. The results indicate that compared with SSB taxes, beverages taxes do cause a much heavier utility loss, and a lower health gain (except for beverage taxes by calorie), which supports the explanation above.

In an Australian context (calibration using Sharma et al.'s estimates), a beverage tax by calories is still the most favorable, but an ad valorem SSB tax is now the least favorable tax base. This is probably because in this case (1) if SSB taxes were imposed, the consumption of fruit juice, milk, tea, and coffee would increase by a large margin, due to strong substitution effects (Sharma et al., 2014), and (2) the untaxed goods (substitutes for SSBs), such as high fat milk, milk drinks, fruit drinks, and fruit juice usually contain more calories than the average over all beverages (Sharma et al., 2014). Consequently, although the loss of utility from SSB taxes is smaller than beverage taxes, the health benefits from SSB taxes are so small that the net welfare increased by SSB taxes is lower than the net welfare created by beverage taxes. As a result, beverage taxes are superior to SSB taxes in this scenario. This explanation also applies for the calibration using estimates from Dharmasena and 
Capps (2012).

Furthermore, given that beverage taxes are superior to SSB taxes when using Sharma et al.'s estimates, and price has the weakest correlation with the harmful ingredients in this scenario, it is reasonable to expect that ad valorem SSB tax becomes the least favorable tax base. Similarly, given that beverage taxes are superior to SSB taxes and volume has the weakest correlation with calories in Dharmasena and Capp's study, a volumetric SSB tax becomes the least favorable tax.

In order to check these arguments, the changes in consumption, the utility loss due to taxes, and the harm reduced by taxes are calculated and reported in Tables $6.2-6.4$ in Appendix V. The results indicate that with Sharma et al.'s estimates, SSB taxes generate substantially higher consumption of high fat milk, a smaller utility loss, and much lower health benefits as expected. Similarly, calculations demonstrate that SSB taxes yield substantially higher post-tax fruit juice consumption, a smaller utility loss, and much lower health benefits using estimates from Dharmasena and Capp's (2011). The results support the explanation outlined in the previous paragraph.

(3) Harm of SSBs

Our estimate showed that the externality of SSBs is $\$ 0.21$ per litre, and the internality of SSBs is NZ\$25.87 per liter (around 121 time larger than externality). These values are thought fairly reasonable as they are consistent with the findings reported by Gruber and Koszegi (2004). They argued that internalities are approximately 100 times greater than the externalities in the case of smoking (Gruber and Koszegi, 2004).

Our estimated harm from externalities is quite low compared with the findings reported in similar studies (Wang et al., 2012; Grummon et al., 2019). Wang et al. (2012) found that one ounce of SSB consumption increased health care costs by approximately one US cent on average, which is equivalent to NZ\$0.51 after adjustment for the exchange rate (Our results suggests that externalities are approximately NZ\$0.21 per litre). Grummon et al. (2019) calculated that the health system externality is around US 0.85 cents per ounce, which equivalent to NZ\$0.45. The difference between their findings and ours could be because compared with New 
Zealand, healthcare costs are much more expensive in the US.

Our estimated harm from internalities is quite low compared with the findings reported by Allcott, Lockwood, and Taubinsky (2019). Having investigated nutrition knowledge and excessive SSB consumption from the Nielsen Homescan data, they found that if people had perfect self-control and were as informed as nutritionists or dietitians, SSB consumption per US household would decrease by 31 to 37 percent. Based on this, they argued that the marginal internality from SSBs is approximately 0.91 to 2.14 cents per ounce on average (Allcott, Lockwood, and Taubinsky, 2019). However, our CV study suggested that the harm from internalities is NZ\$10.35 per litre which is equivalent to US\$0.2 per ounce after being adjusted by exchange rate. The substantial difference between our figures and theirs could be because that dietician may value their health higher than an average person does. As a result, there is risk that the harm from internalities is overestimated by Allcott, Lockwood, and Taubinsky.

(4) Policy Implications

Policy implications of the study include the following.

First, given that such taxes are beneficial to society, SSB taxes or sugary beverage taxes should be introduced in New Zealand. This is probably feasible as SSB taxes have been supported by the public (Herald on Sunday, 2016; Jacobson \& Brownell, 2000).

Second, it would be desirable to tax all sugary beverages by calories, though it may be somewhat less convenient compared with ad valorem taxes and volumetric taxes in reality. However, given that a 0.03 rupees/gm sugar SSB tax was applied in Mauritius, and a 2.1cents/gm sugar tax on sugary beverage was imposed in South Africa (Cawley et al., 2019), the proposal does seem to be quite feasible.

In addition, current SSB taxes probably should be increased by a large margin, as they are now somewhat close to what the optimal level would be if only externalities were addressed. In our estimation, when both externalities and internalities are legitimate concerns, the optimal level of volumetric taxes will be approximately NZ\$6 to NZ\$7 per litre, and the optimal level of ad valorem taxes is around $120 \%$ to $210 \%$ in New 
Zealand. Studies suggested that many SSB taxes have failed to reach their policy goals because the tax rates were too low (Colantuoni et al. (a), 2015; Colantuoni et al. (b), 2014). As a result, consumers may fail to notice the price increase (Colantuoni et al. (a), 2015). Moreover, given that a 100\% excise tax has been imposed in Bahrain and Saudi Arabia (Cawley et al., 2019), such a high tax rate is believed fairly feasible. Finally, taxing only SSBs involves a risk that consumers will switch to untaxed beverages, possibly those with high calories. As suggested by the simulation results, fruit juice and high fat milk which contain high calories have a strong substitute relationship with SSBs in Australia and the USA. Therefore, the consumption of fruit juice and high fat milk may increase substantially if only SSB taxes are imposed. Consequently, SSB taxes may not be very effective and even fail to reach their policy goals. Perhaps this risk can be partially reduced if SSB taxes are combined with other interventions, such as regulations on branded cool packaging, limits to convenience, and clear and prominent calorie content labeling for all beverages.

(5) Limitations \& Future Work

The approach taken in the study effectively assumes quasi-linear utility. This is convenient because the welfare measure is equivalent to DWL, which allows a variety of empirical results to be organized in a common framework. The assumption does imply that the Slutsky matrix must be symmetric and negative semi-definite. Without negative semi-definiteness, the deadweight loss measure can be negative. However, the Slutsky matrix calculated using the Ni Mhurchu et al.' estimates and Dharmasena and Capps's estimates are not negative semi-definite, though the following modifications have been made to the matrix. First, elasticities were set equal to zero if those estimates were not statistically significant. Second, we impose symmetry by taking an average of the slope matrix and its transpose. But one of the three eigenvalues of the Slutsky matrix in Ni Mhurchu et al.'s study is still positive, and two of the ten eigenvalues in Dharmasena and Capps's study are positive. Since the simulation results are fairly reasonable, it is hard to determine how the results were affected by this anomaly. Fortunately, data from Sharma et al.'s study does not have this problem. Given that the main conclusions are drawn from the findings of all three 
studies, and these results of these three studies are quite logically consistent, this problem may not be a significant threat and therefore, our findings are thought probably to be reasonably reliable and fairly robust.

Second, we do not capture the effects of SSB taxes on beverages consumed in the restaurants. This problem is somewhat inevitable due to lack of reliable data sources in New Zealand. However, this concern is moderated by the fact that food consumed in restaurants only account for a small portion of total energy intake (Ni Mhurchu et al., 2013). Given this, this weakness may not be a real threat to our findings.

Third, the value of the parameter $\beta$ which reveals the proportion of health and health-related harm accounted by consumers is taken from a paper that studies a dynamic job search model (Paseman, 2008). As discussed previously, consumers are assumed to account for only a proportion of health harm due to discounting and lack of self-control. However, the value of $\beta$ estimated by Paseman (2008) only includes the effects of discounting. This concern is somewhat moderated, given that existing models of sin goods assume a similar value. Having investigated nutrition knowledge and excessive SSB consumption from 18,000 households, Allcott, Lockwood, and Taubinsky (2019) found that if people had perfect self-control and were acknowledged as nutritionists or dietitians, SSB consumption per US household would decrease by 31 to 37 percent $(1-\beta=0.37$, so that $\beta=0.63)$. This estimate is quite close to the value used in our study.

As discussed in the first chapter, the association between heavy SSB consumption and obesity and diabetes has been identified in several studies (Hu, 2013). However, whether taxes alone can reduce SSB consumption and further generate health benefits is controversial. To effectively improve health, such a tax must achieve four steps (Wilson and Hogan, 2017). Firstly, an increase in prices must be seen after such a tax is imposed. Secondly, such an increase in price has to result in a decrease in consumption. Third, the reduction in consumption must decrease people's sugar intake. Finally, the decrease in energy intake has to lower risk factors (e.g., obesity). In our model, we implicitly assumed that those four steps can be reached. But in reality, a drawback in any of these five steps will make an intervention fail to reach its 
policy goals. Third, redistributional concerns are not acknowledged in this study, despite that they have been prominent in some previous works (Sharma, 2014; Allcott, Lockwood, and Taubinsky, 2019). Allcott, Lockwood, and Taubinsky (2019) have shown that $\sin$ tax regressivity can be offset by income tax reforms if differences in consumption are due to income effects, rather than preference heterogeneity. The other solution to this equity problem could be subsidies for healthy foods, especially those low energy and nutrient beverages, for instance, low-fat milk. Given this, there is a need for future studies to model the effects of subsidies for healthy food which is used as a targeted transfer of SSB taxes revenue.

In addition, the effects of taxes on different subgroups are not investigated in this study because of lack of available data sources in New Zealand. When subgroup data becomes available, it would be meaningful to investigate the equity concerns across different tax bases by calculating concentration indices.

Finally, prices and expenditures would be endogenous if there is unobserved demand heterogeneity or local demand shocks, so there may be potential bias in estimating those structural equations in the study conducted by Ni Mhurchu et al. As a result, the optimal tax levels estimated in the base case can be somewhat biased. As discussed in the literature review section, one potential solution to solve this problem is to find instruments associated with prices and expenditures but uncorrelated with unobserved demand heterogeneity and shocks. One plausible instrument suggested is the average price of each product across other markets if the market pricing factors are independent across markets (Harding \& Lovenheim, 2017). Another possible approach to isolate quasi-random variation in non-experimental data is to try to control for product quality and demand fluctuations. However, no New Zealand studies applied either of the potential solutions. Therefore, it would be meaningful to conduct a simulation in the future to check the reliability of the estimated optimal tax rates when such data becomes available.

\subsection{Conclusion}

In this chapter, we calculate that the portion of marginal harm that people do not take into account is approximately $\$ 10.35$ per litre which is equivalent to $\$ 0.0310$ per 
calorie. The marginal harm associated with externality is $\$ 0.21$ per litre which is equivalent to $\$ 0.0006$ per calorie. Given the harm from internalities and externalities, the total harm can be estimated as a summation of the two, which is $\$ 10.56$ per litre and equivalent to $\$ 0.032$ per calorie.

When marginal harm is $\$ 0.032$ per calorie, the socially optimal levels for a per litre SSB tax, an ad-valorem SSB tax, and a SSB tax by calorie are 7.31, 2.09, and 0.032, respectively. The socially optimal rates for a per litre beverage tax, an ad-valorem beverage tax, and a beverage tax by calorie are $6.50,1.21$, and 0.032 , respectively.

All these taxes increase welfare from the original point where no tax is imposed, which indicates that SSB taxes are beneficial to society. A beverage tax by calories is the most favorable option as the tax base has a perfect relationship with the harmful ingredients. Whether taxing all beverages by price or by litres are better than taxing SSBs only, depends on how strong the substitution effects are, and how much harmful ingredients substitution contain. Our findings are thought to be reasonably reliable and robust.

Given the findings suggested by the simulation results, SSB taxes or beverage taxes are beneficial to society, so they should be introduced to New Zealand. It would be desirable to tax all sugary beverages by calories, though it may be somewhat less convenient compared with ad valorem taxes and volumetric taxes in reality. In addition, for countries where a SSB tax or beverage tax has been imposed, their tax rates probably should be increased by a large margin. Finally, taxing only SSBs involves a risk that consumers will switch to untaxed beverages, possibly those with high calories. Perhaps this risk can be partially reduced if the calorie content is labeled clearly and prominently. 


\section{Final Conclusion}

Previous studies have shown that excess sugar intake is a potential cause of obesity and diabetes, and one of the major sources of sugar is SSBs (Adair et al., 2016; Parmenter, Jordan \& Jayasinghe, 2017; Gustavsen \& Rickertsen, 2011; Ludwig, 2001). Therefore, SSB taxes have been applied as an intervention against obesity and diabetes (Bleich et al., 2013; Burgess et al., 2017). However, such an intervention may not be effective, if consumers switch to other sugary foods which would be substitutes for SSBs (Fletcher, 2010). In addition, little is known about whether a $20 \%$ tax rate, or another tax rate, is socially optimal or not.

The literature review found that the socially optimal level of SSB taxes has been studied by Allcott, Lockwood, and Taubinsky (2019); but they also left room for future studies to investigate different tax bases at their socially optimal levels, with considerations of substitutes and complements. Grummon et al. (2019) compared different tax bases under the 'economically equivalent taxes' assumption, but there is still a need for a full discussion of the different tax bases at socially-optimal levels, without assuming they are "economic equivalent taxes". Besides, it may be valuable to investigate whether taxing all beverages is superior to taxing SSBs only. Finally, a need of measuring internalities and externalities from SSBs in New Zealand contexts is identified in the literature review.

Following the findings of the literature review, this study examines which tax base is the most socially efficient at the socially optimal rate, taking into account substitutes and complements. We also investigate which tax base is the most socially effective, and whether taxing all beverages is superior to taxing only SSBs. We start from a simple benchmark model of the optimal corrective tax based on the neoclassical approach to consumer choices, which is familiar from Allcott, Lockwood, and Taubinsky (2019) but without redistributional concerns. The familiar result is that the 
corrective tax should be set equal to the portion of the marginal harm that the consumer does not account for. Then an extended model is introduced, which considers different commodities and allows substitution and complementary effects, followed with an analysis of the tax impact. The results indicate that the optimal corrective tax can be expressed in terms of three terms: 1) the proportion of harm that is not reflected in consumption, 2) the marginal harm of consumption decisions, and 3) a scaler for the tax which reflects tax impacts.

Since the marginal harm from SSBs is included as a component of the optimal tax formula, contingent valuation was applied to estimate the monetary value of harm associated with internalities; and cost analyses using a Markov model and the UKPDS model are conducted to measure the harm associated with externalities.

The CV study investigated 293 Facebook users in Wellington. Results indicate that the marginal WTP for 1\% reduction in diabetes risk, stroke risk and heart disease risk are NZ\$404.86, NZ\$809.04, and NZ\$1,236.84, respectively. Given the marginal WTP and SSB consumption per person per year, the marginal harm the average drinker takes into account is NZ\$15.52 per litre. However, due to the relatively small sample size and hypothetical bias, the findings of the CV study should be interpreted with some caution, when applied to the general population of New Zealand.

Following the findings of this $\mathrm{CV}$ study above and the measurement conducted in previous studies that people may only account for $60 \%$ of the total health harm due to hyperbolic discounting, we estimate that the harm from internalities that is not taken into account for is approximately NZ\$10.35 per litre. It is equivalent to NZ\$0.0310 per calorie.

Next, the cost analysis study demonstrates that the point estimate of the harm of an IHD, a stroke, and diabetes associated with SSB consumption are NZ\$0.017 per litre, NZ\$0.148 per litre, and NZ\$0.045 per litre respectively. The findings are considered sound and quite reliable, as they are very close to the findings of a similar study conducted by the NZIER. Given the figures listed above, the marginal harm from externalities is approximately $\$ 0.21$ per litre which is equivalent to $\$ 0.0006$ per calorie. Our estimates of internality and externality are thought reasonable as they are 
consistent with the findings reported by Gruber and Koszegi (2004). They argued that internalities are approximately 100 times greater than the externalities in the case of smoking (Gruber and Koszegi, 2004).

Finally, the total harm can be calculated as a summation of the costs from both internalities and externalities. This would be $\$ 10.56$ per litre and equivalent to $\$ 0.032$ per calorie. When marginal harm is $\$ 0.032$ per calorie, the socially optimal levels for a per litre SSB tax, an ad-valorem SSB tax, and a SSB tax by calorie are 7.31, 2.09, and 0.032 respectively. The socially optimal levels for a per litre beverage tax, an ad-valorem beverage tax, and a beverage tax by calorie are 6.50, 1.21, and 0.032 respectively. Our estimate of the optimal tax rate suggests that prices of SSBs probably should increase by $100 \%$ to $200 \%$ in New Zealand. The estimated optimal tax rates above are quite high compared with the SSB or sugary taxes that have been imposed in the real world. Tax rates that have actually been imposed are reasonably close to what the optimal rate would be, if only externalities (but not internalities) had to be corrected. This may be because it is more controversial for government to intervene to address internalities than it is for externalities.

Next, a quadratic functional form for utility is constructed to measure the welfare change associated with SSB taxes. Results demonstrate that all the taxes increase welfare from the original point where no tax is imposed, which indicates that SSB taxes are beneficial to society and therefore, should be introduced in New Zealand. A beverage tax by calories is always the most favorable option, as it has a perfect relationship with the harmful ingredients and yields the highest welfare improvement. This is consistent with the findings reported by Grummon et al. (2019) and Zhen et al. (2014).

In addition, whether taxing all beverages by price or by litres is better than taxing only SSBs depends on how strong the substitution effects are, and how many calories substitutes contain. When substitutes contain low energy and substitute effects are strong, taxing SSBs is probably better than taxing all beverages by price or by litres, and the socially optimal tax rate will become larger if substitutes and complements are considered. 
There are several policy applications of the findings of this study. First, because SSB taxes and sugary beverage taxes are beneficial to society, they should be introduced in New Zealand. Given that SSB taxes are supported by the public in New Zealand (Herald on Sunday, 2016; Jacobson \& Brownell, 2000), such a policy is likely to be politically feasible. Second, it would be desirable to tax all sugary beverages by calories, though it may be less convenient compared with ad valorem taxes and volumetric taxes in reality. However, because a 0.03 ruppees/gm sugar SSB tax has been applied in Mauritius, and a 2.1cents/gm sugar sugary beverage taxes has been imposed in South Africa (Cawley et al., 2019), the proposal should be feasible in New Zealand, too.

In addition, current SSB taxes imposed in other countries probably should be increased by a large margin, as they seem to be substantially lower than the optimal level, assuming that internalities are a legitimate consideration for tax design. In our estimation, when both externalities and internalities are addressed, the optimal level of volumetric taxes will be approximately NZ\$6 to NZ\$7 per litre, and the optimal level of ad valorem taxes is around $120 \%$ to $210 \%$ in New Zealand. One potential justification for a high tax rate is that many SSB taxes fail to reach their policy goals in reality as tax rates are too low to yield a substantial reduction in SSB consumption. Finally, taxing only SSBs involves a risk that consumers will switch to untaxed beverages, possibly those with high calories. As suggested by the simulation results, fruit juice and high fat milk which contain high calories have a strong substitute relationship with SSBs in Australia and the USA. Therefore, the consumption of fruit juice and high fat milk may increase substantially if only SSB taxes are imposed in those countries. Consequently, SSB taxes may not be very effective and even fail to reach their policy goals. Perhaps this risk can be partially reduced if SSB taxes are combined with other interventions, such as regulations on branded packaging, limits to convenience, and clear and prominent calorie content labeling for all beverages.

As with Allcott, Lockwood, and Taubinsky (2019), our model addresses the optimal levels with consideration of substitutes and complements. The main difference between our study and their paper is that our study compares different tax bases at 
their socially optimal levels, while Allcott, Lockwood, and Taubinsky (2019) focus on equity concerns. In addition, we also investigate whether SSB taxes are superior to beverage taxes.

Different tax bases are compared by Zhen, Brissette \& Ruff (2014). They used a fully modified distance metric model of differentiated product demand which endogenizes the cross-price effects, with consideration of substitutes and complements. Compared with their study, our model moves a step forward from applying a theoretical method to compare different tax bases at their socially optimal levels. This makes a real contribution because only when different tax bases are compared at their socially optimal levels can we determine which tax base is the most favorable. Compared with Grummon et al.'s study (2019) in which different tax bases are compared under the "economically equivalent taxes" condition, our model dropped this assumption and therefore moves a step forward. The "economically equivalent taxes" is defined as those that impose the same tax rate to SSBs with average sugar content. The main concern about this assumption is that as government is assumed to choose a tax rate maximizing social welfare given a tax base, it is meaningless to assume the same tax rate. Besides, ad valorem tax is compared with other tax bases in our study. We believe this is meaningful as ad valorem taxes are probably the most commonly tax base for SSB taxes. Finally, another advantage of our model is that our model allows for the effects of complements and substitutes.

Compared with those CUA and CBA studies, the strength of our study is that the WTP values probably include not only the health harm of diseases, but also count other costs such as the loss in productivity, time costs, travel costs and emotional harm of relatives and friends due to sickness. Moreover, although health benefits (e.g., QALY gain or DALY reduction) are calculated as benefits of SSB taxes in many previous studies, almost none of them further link health changes to internalities. This matters as consumers do consider a proportion of harm of SSBs, rather than ignore all of it when they are purchasing and consuming SSBs. As a result, benefits of taxes measured in these studies are highly likely to be overestimated. In our study, this problem is somewhat solved by assuming that consumers account for a proportion of 
health harm in purchasing SSBs. Furthermore, our study also modeled the loss in utility due to SSB taxes which is seldom mentioned and discussed in the CUA/CBA literature and public health studies. Our approach is to develop a welfare measurement to evaluate the change due to SSB taxes. This method is relatively simple but robust. It is equivalent to using a multi-commodity deadweight loss measure, with linear approximations of demand curves. Given this, our study moves a step forward again. Finally, this study is probably the first study which estimates the externalities and internalities of SSBs in New Zealand. Although Allcott, Lockwood, and Taubinsky (2019) and Wang et al. (2015) estimated externalities from summarizing previous scholars' work, the data source they used is unlikely to be applicable for New Zealand, due to the heterogeneities in healthcare systems and costs cross countries. Also, there may be a bias in the value of internalities estimated in Allcott, Lockwood, and Taubinsky's study. They measured internalities by comparing the SSB consumption consumed by dieticians with non-health professionals. Because people who are more concerned about health are more likely to become dieticians, there may be a selection bias in their study. This weakness leads to a risk that the harm from internalities is overestimated by Allcott, Lockwood, and Taubinsky. Given the arguments discussed above, our work probably makes a real contribution to this little-known topic by estimating the harm of SSBs, using the methods which have never been applied in previous studies.

The weaknesses of the study include the following. First, income effects of SSB taxes are ruled out in this study. This concern is somewhat moderated, because we think that strong income effects are very unlikely for soft drinks, given that SSB consumption only account for a very small proportion of total expenditure in New Zealand (Ni Mhurchu et al., 2013).

Second, in chapter 3, we assume that utility is linear in money. This assumption also indicated that consumers are risk neutral about the possibility of income loss, which might contradict the fact that in real life people often purchase insurance against loss of income. Therefore, it would still be meaningful to drop the quasi-linear utility assumption and include income effects in a similar study in the future. 
Third, the model assumes a representative agent. However, there can be substantial differences between consumers in reality. For example, there may be heterogeneities in marginal harm parameters and the degree self-control. In order to address this problem, in the future I plan to study a model with a distribution of consumers.

Fourth, we assumed that SSBs are provided by perfectly competitive firms, so that the tax would be $100 \%$ passed to consumers in our model. This assumption is at odds with the fact that the beverage industry is probably oligopolistic (e.g., Coca Cola and Pepsi) and therefore, prices of SSB far exceed their marginal costs. Consequently, as discussed in the literature review, the pass-through rates varies from around $50 \%$ to $100 \%$ in the real world (Falbe et al.; 2015; Colantuoni, 2014). In addition, market power may have a substantial effect on the optimal tax rate, given that Calcott and Petkov (2013) have shown that the optimal tax rates on cigarettes in an oligopolistic market would decrease if market power were considered. Therefore, there is a need for future studies to derive the marginal cost function if such data is available, and firms' strategic responses to the imposed taxes should also be considered.

Another concern of study is that we do not consider heterogeneities among similar sugary beverages. For instance, consumers may switch from Pepsi to some lower price local brands after such taxes are imposed. If those lower prices soft drinks contain higher amount sugar than Pepsi does, such a tax may fail to reach its policy goals. Therefore, the substitutes within similar products probably should be simulated in the future when such data becomes available in New Zealand.

In addition, as with many studies included in the literature review, our model does not capture the effects of taxes on food in restaurants, due to lack of available data in New Zealand. This concern is somewhat moderated by the argument that food in restaurants, especially beverages, only account for a very small proportion of the whole food expenditure in New Zealand (Ni Mhurchu et al., 2013).

Some authors claim that sugar has addictive properties (Wilson and Hogan, 2017). Although this claim is controversial, it is possible that such an effect might change the optimal tax rate. If consumers are addicted to SSBs, they will be less responsive to price changes, and their future consumption will depend on their consumption today. 
The more they drink today, the more they will consume in the future (Wilson and Hogan, 2017). In addition, if SSBs are addictive, there will be substantial differences between short-term elasticities (when there is a one-off price change) and long-term elasticities (when there is a permanent price change). In order to address this problem, a dynamic model may be constructed in the future.

Because people with lower socioeconomic status are more likely to be intensive consumers whom the main tax burden will come to, SSB taxes are highly likely to cause an equity issue (Jacobson \& Brownell, 2000). On the other hand, lower socioeconomic status people are more responsive to price changes, so their SSB consumption will decrease more significantly than the consumption of their counterparts with higher socioeconomic status (Lal et al., 2017). Given the two considerations listed above, the regressivity is probably not a severe problem. In order to verify this argument, a simulation using different socioeconomic class data should be conducted to check the effects of taxes on different subgroups in the future, when reliable data becomes available. In addition, the equity concerns of SSB taxes possibly can be addressed by calculating concentration indices.

Furthermore, we assume that the total harm of consuming soft drinks is proportional to the total amount of calories in this study. However, studies have shown that carbon dioxide could reduce calcium absorption and thereby, damaging bone health, causing dental problems and other related diseases (Kim et al., 2017; Malik, 2017). Another concern is that some zero-calorie SSBs may contain aspartame or stevia which may be harmful to human health. Since the association between aspartames intake and some diseases (e.g., migraine and muscle problems) has been identified in a few studies, future studies in similar area should take these factors into consideration, accounting for all the potential harmful ingredients in SSBs (Tandel, 2011; Narain, Kwok and Mamas, 2016).

As discussed in the first chapter, the association between intensive SSB consumption and obesity and diabetes has been identified in many studies ( $\mathrm{Hu}, 2013)$. However, whether taxes alone can reduce SSB consumption and further generate health benefits is controversial. In order to effectively improve health, such a tax must achieve four 
steps (Wilson and Hogan, 2017). Firstly, an increase in prices must be seen after such a tax is imposed. Secondly, such an increase in price has to result in a decrease in consumption. Third, the reduction in consumption must decrease people's sugar intake. Finally, the decrease in energy intake has to lower risk factors (e.g., obesity) (Wilson and Hogan, 2017). Failure to complete any of these four steps will prevent the intervention from reaching its policy goals. In this study, we implicitly assume that those four steps can be well reached. Given this, the benefits are probably overestimated.

In addition, our estimation of harm from internalities is made under the assumption of constant marginal WTP for each unit of risk reduction. However, there is still insufficient evidence to support this assumption.

The redistribution of tax revenue is not addressed in this study. Allcott, Lockwood, and Taubinsky (2019) have shown that sin tax regressivity can be offset by income tax reforms if differences in consumption are due to income effects, rather than preference heterogeneity. Besides income tax reforms, the other redistribution could be subsidies for healthy foods. Such subsidies are crucial and useful because (1) the income effects of SSB taxes may decrease healthy food consumption (although we think it should be quite small) and (2) given the substitution relationship between sugary drinks and those healthy foods, such subsidies may further reduce SSB consumption as a result of decrease in healthy food price. Therefore, there is probably a need for future studies to model the effects of subsidies for healthy food that can be used as a targeted transfer of SSB taxes.

Imposing taxes will inevitably generate some cost. For example, government/industry administration and compliance time costs, audit direct costs, tax certification system operating costs, legislation cost, administration cost, and costs for policy advice. Having considered the possible effects of SSB substitutes and the heterogeneity of consumers, no matter how low the costs of implementation, there is always a risk that SSB taxes are counterproductive if costs are greater than benefits. Therefore, there is probably a need to conduct a CBA of SSB taxes and sugary drink taxes which explicitly includes these costs in the future. 
Finally, the welfare change associated with SSB taxes is measured using a quadratic utility function in this study, so that the deadweight loss is calculated as the decrease in utility plus tax revenue. To the extent that demand curve deviate from being linear, this approach will involve some approximation errors. The other potential approach to measure the deadweight loss is to ask consumers' willingness-to-accept for the reduction of SSB consumption. 


\section{Appendix I: Literature Review}

Table 2.1: Basic Information on Non-Experiment Studies

\begin{tabular}{|c|c|c|c|c|c|c|}
\hline First Author & Year & Country & $\begin{array}{l}\text { Type and Rate } \\
\text { of Tax }\end{array}$ & Main Focus & Data Sources & $\begin{array}{l}\text { Approach \& Statistical } \\
\text { Analysis }\end{array}$ \\
\hline Allcott & 2017 & USA & $\begin{array}{l}\text { Socially optimal } \\
\text { level }\end{array}$ & Redistribution & $\begin{array}{l}\text { consumer panel data; 2009-2010 National Health } \\
\text { and Nutrition Examination Survey }\end{array}$ & $\begin{array}{l}\text { Utility maximization model } \\
\& \text { simulation }\end{array}$ \\
\hline Bonnet & 2013 & France & $\begin{array}{l}\text { A } 9 \text { cents per } \\
\text { litre excise tax }\end{array}$ & $\begin{array}{l}\text { Heterogeneity of tax } \\
\text { burden \& income }\end{array}$ & $\begin{array}{l}2005 \text { data from a French representative consumer } \\
\text { panel data }\end{array}$ & Structural form estimation \\
\hline Briggs (a) & 2013 & UK & $\begin{array}{l}20 \% \text { tax rate and } \\
100 \% \text { pass - } \\
\text { through rate }\end{array}$ & $\begin{array}{l}\text { Heterogeneity of tax } \\
\text { burden \& income }\end{array}$ & $\begin{array}{l}\text { Living Costs and Food Survey, 2010; National } \\
\text { Diet and Nutrition Survey (2008-10); Health } \\
\text { Survey for England, 2010, and the Scottish } \\
\text { Health Survey, } 2010\end{array}$ & $\begin{array}{l}\text { A Bayesian approach to } \\
\text { estimate an almost ideal } \\
\text { demand system; the PRIME } \\
\text { model }\end{array}$ \\
\hline Briggs (b) & 2013 & Ireland & $\begin{array}{l}10 \% \text { tax rate and } \\
100 \% \text { pass - } \\
\text { through rate }\end{array}$ & Effects on obesity & $\begin{array}{l}2007 \text { Survey on Lifestyle and Attitude to } \\
\text { Nutrition }\end{array}$ & $\begin{array}{l}\text { A comparative risk } \\
\text { assessment model }\end{array}$ \\
\hline Cobiac & 2017 & Australia & $\$ 0.47 / 1$ & Cost -effectiveness & $\begin{array}{l}\text { Online data of Woolworths Supermarket in June } \\
\text { 2011; Australian Health Survey data; Asia Pacific } \\
\text { Cohort Study; Australian Institute of Health and } \\
\text { Welfare data; NZ data }\end{array}$ & Simulation \\
\hline Colchero (a) & 2015 & Mexico & N/A & $\begin{array}{l}\text { Consumptions } \\
\text { \&elasticity }\end{array}$ & The MNHIES & AIDS, linear regression \\
\hline Creedy & 2016 & $\begin{array}{l}\text { New } \\
\text { Zealand }\end{array}$ & $\mathrm{N} / \mathrm{A}$ & Substitutes & $\mathrm{N} / \mathrm{A}$ & $\begin{array}{l}\text { Modeling } \\
\text { simulation }\end{array}$ \\
\hline
\end{tabular}




\begin{tabular}{|c|c|c|c|c|c|c|}
\hline Dharmasena & 2014 & USA & $\begin{array}{l}10 \% \text { ad valorem } \\
\text { tax }\end{array}$ & General Equilibrium & Nielsen Homescan (NH) scanner data & Simulation \\
\hline Etile & 2015 & Australia & $\begin{array}{l}20 \text { cts } / 1 \text { excise } \\
\text { tax; } 20 \% \text { ad } \\
\text { valorem tax }\end{array}$ & $\begin{array}{l}\text { Heterogeneity of tax } \\
\text { burden \& income }\end{array}$ & 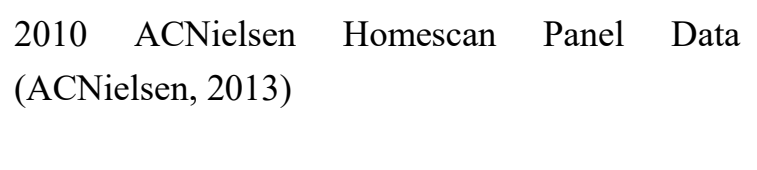 & $\begin{array}{l}\text { A finite mixture } \\
\text { instrumental variable Tobit } \\
\text { model }\end{array}$ \\
\hline Finkelstein & 2013 & USA & $\begin{array}{l}20 \% \text { tax rate and } \\
100 \% \text { pass }- \\
\text { through rate }\end{array}$ & Substitutes & 2006 Homescan panel & $\begin{array}{l}\text { Instrumental variables, } \\
\text { quantile regression }\end{array}$ \\
\hline Griffith & 2018 & UK & $\begin{array}{l}\text { Volumetric } \\
\text { optimal tax rate }\end{array}$ & $\begin{array}{l}\text { Optimal tax rate \& } \\
\text { redistribution }\end{array}$ & N/A & Utility maximization model \\
\hline Guerrero-López & 2017 & Chile & $\begin{array}{l}10 \% \text { increase in } \\
\text { price }\end{array}$ & Price elasticity & VII Family Budget Survey 2012-2013 & AIDS \\
\hline Kifer & 2015 & USA & $\$ 0.02$-per-ounce & $\begin{array}{l}\text { Heterogeneity of tax } \\
\text { burden \& income }\end{array}$ & $\begin{array}{l}\text { Nielsen Homescan panel; store-level data from } \\
\text { Chicago grocery store chain Dominick's Finer } \\
\text { Foods }\end{array}$ & $\begin{array}{l}\text { Hierarchical } \\
\text { model }\end{array}$ \\
\hline Lal & 2017 & Australia & $\begin{array}{l}20 \% \text { tax rate and } \\
100 \% \text { pass }- \\
\text { through rate }\end{array}$ & $\begin{array}{l}\text { Heterogeneity of tax } \\
\text { burden \& income }\end{array}$ & Australian Health Survey 2011-2012 & $\begin{array}{l}\text { Simulation: Markov cohort } \\
\text { models }\end{array}$ \\
\hline Long & 2015 & USA & $\$ 0.01 /$ ounce & $\begin{array}{l}\text { Cost-effectiveness of } \\
\text { a SSB tax }\end{array}$ & $\begin{array}{l}2012 \text { National Health and Nutrition Examination } \\
\text { Survey }\end{array}$ & $\begin{array}{l}\text { Simulation; } \\
\text { Cost-effectiveness analysis }\end{array}$ \\
\hline Mekonnen & 2013 & $\begin{array}{l}\text { California, } \\
\text { USA }\end{array}$ & $\begin{array}{l}\text { Penny-per-ounce } \\
\text { excise tax }\end{array}$ & Effects on CVD & $\begin{array}{l}\text { Western region participants in NHANES, years } \\
\text { 1999-2008, and from the California Health } \\
\text { Interview Survey (CHIS), years 2001-2009 }\end{array}$ & $\begin{array}{l}\text { Simulation: } \\
\text { Cardiovascular } \\
\text { Policy Model }\end{array}$ \\
\hline
\end{tabular}




\begin{tabular}{|c|c|c|c|c|c|c|}
\hline Manyema (a) & 2014 & $\begin{array}{l}\text { South } \\
\text { Africa }\end{array}$ & $\begin{array}{l}20 \% \text { increase in } \\
\text { price }\end{array}$ & Effects on obesity & $\begin{array}{l}\text { Consumption data from the } 2012 \text { SA National } \\
\text { Health and Nutrition Examination Survey, } \\
\text { National Income Dynamics Survey } 2012 \text { and a } \\
\text { previous meta-analysis of studies on own- and } \\
\text { cross-price elasticities of SSBs }\end{array}$ & Simulation \\
\hline Manyema (b) & 2016 & $\begin{array}{l}\text { South } \\
\text { Africa }\end{array}$ & $\begin{array}{l}20 \% \text { tax rate and } \\
100 \% \text { pass - } \\
\text { through rate }\end{array}$ & $\begin{array}{l}\text { Effects on stroke and } \\
\text { stroke-related costs }\end{array}$ & $\begin{array}{l}\text { Data from the } 2012 \text { South African National } \\
\text { Health and Nutrition Examination Survey } \\
\text { (SANHANES-1) }\end{array}$ & Simulation \\
\hline Marron & 2015 & N/A & $\begin{array}{l}\text { Volumetric } \\
\text { optimal tax rate }\end{array}$ & $\begin{array}{l}\text { Optimal tax rate \& } \\
\text { redistribution }\end{array}$ & N/A & $\begin{array}{l}\text { Utility maximization model } \\
\& \text { simulation }\end{array}$ \\
\hline Schwendicke & 2017 & Germany & $\begin{array}{l}20 \% \text { tax rate and } \\
100 \% \text { pass }- \\
\text { through rate }\end{array}$ & $\begin{array}{l}\text { Effects on obesity; } \\
\text { heterogeneity of tax } \\
\text { burden \& income }\end{array}$ & II German Nutrition Survey (NSV II) & Simulation \\
\hline Sharma & 2014 & Australia & $\begin{array}{l}\text { A } 20 \% \text { sale tax } \\
\text { and a } 20 \text { cent } / \mathrm{L} \\
\text { volumetric tax }\end{array}$ & $\begin{array}{l}\text { Heterogeneity of tax } \\
\text { burden \& income }\end{array}$ & 2011 Household level scanner data & AIDS \\
\hline Sánchez-Romero & 2016 & Mexico & N/A & $\begin{array}{l}\text { Effects on diabetes } \\
\text { and CVDs }\end{array}$ & $\begin{array}{l}\text { Government and market research surveys and } \\
\text { public healthcare institutions }\end{array}$ & $\begin{array}{l}\text { Cardiovascular Disease } \\
\text { Policy Model-Mexico }\end{array}$ \\
\hline Tiffin & 2015 & UK & $\begin{array}{l}0.06 \text { pounds / } \\
\text { litre }\end{array}$ & $\begin{array}{l}\text { Heterogeneity of tax } \\
\text { burden \& income }\end{array}$ & $\begin{array}{l}2010 \text { Living Costs and Food Survey; KANTAR } \\
\text { World Panel }\end{array}$ & AIDS \\
\hline Veerman & 2016 & Australia & $20 \%$ valoric tax & $\begin{array}{l}\text { Effects on health and } \\
\text { healthcare costs }\end{array}$ & Australian Health Survey (AHS) 2011-2013; & Simulation \\
\hline Wang & 2015 & USA & $\begin{array}{l}10 \% \text { sales tax; a } \\
\text { penny-per-ounce } \\
\text { tax }\end{array}$ & Dynamic & $\begin{array}{l}\text { Weekly scanner data from } 2002 \text { to } 2004 \text { provided } \\
\text { by Information Resources Inc. (IRI). }\end{array}$ & Modeling and simulation \\
\hline Zhen (a) & 2014 & New York & 0.04 -cent & By ounce or by & Nielsen Scan Track scanner data & AIDS \\
\hline
\end{tabular}


half-cent per Heterogeneity of tax A censored Exact Affine Stone Index incomplete AIDS 
Table 2.2: Main Findings of Non-Experiment Studies

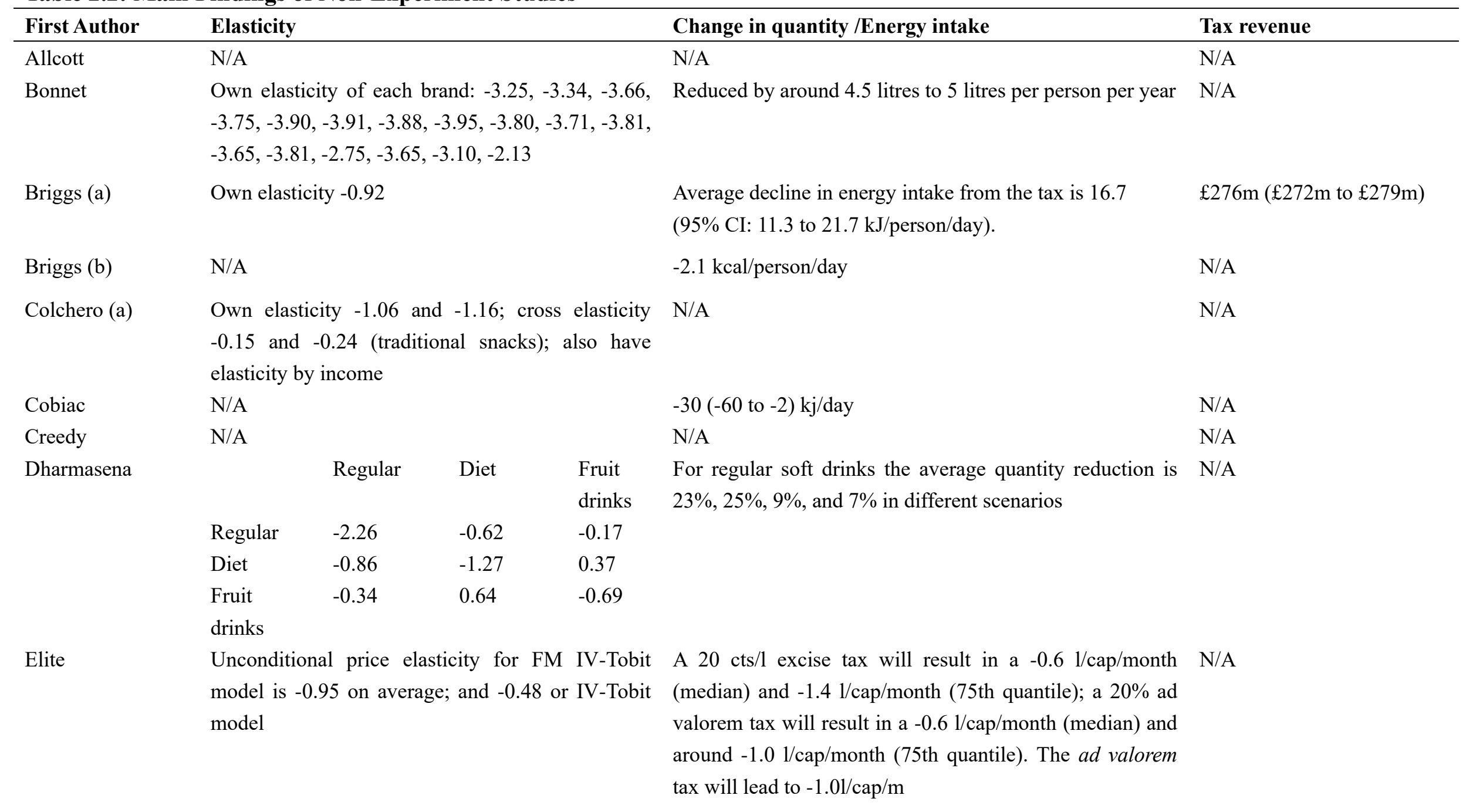




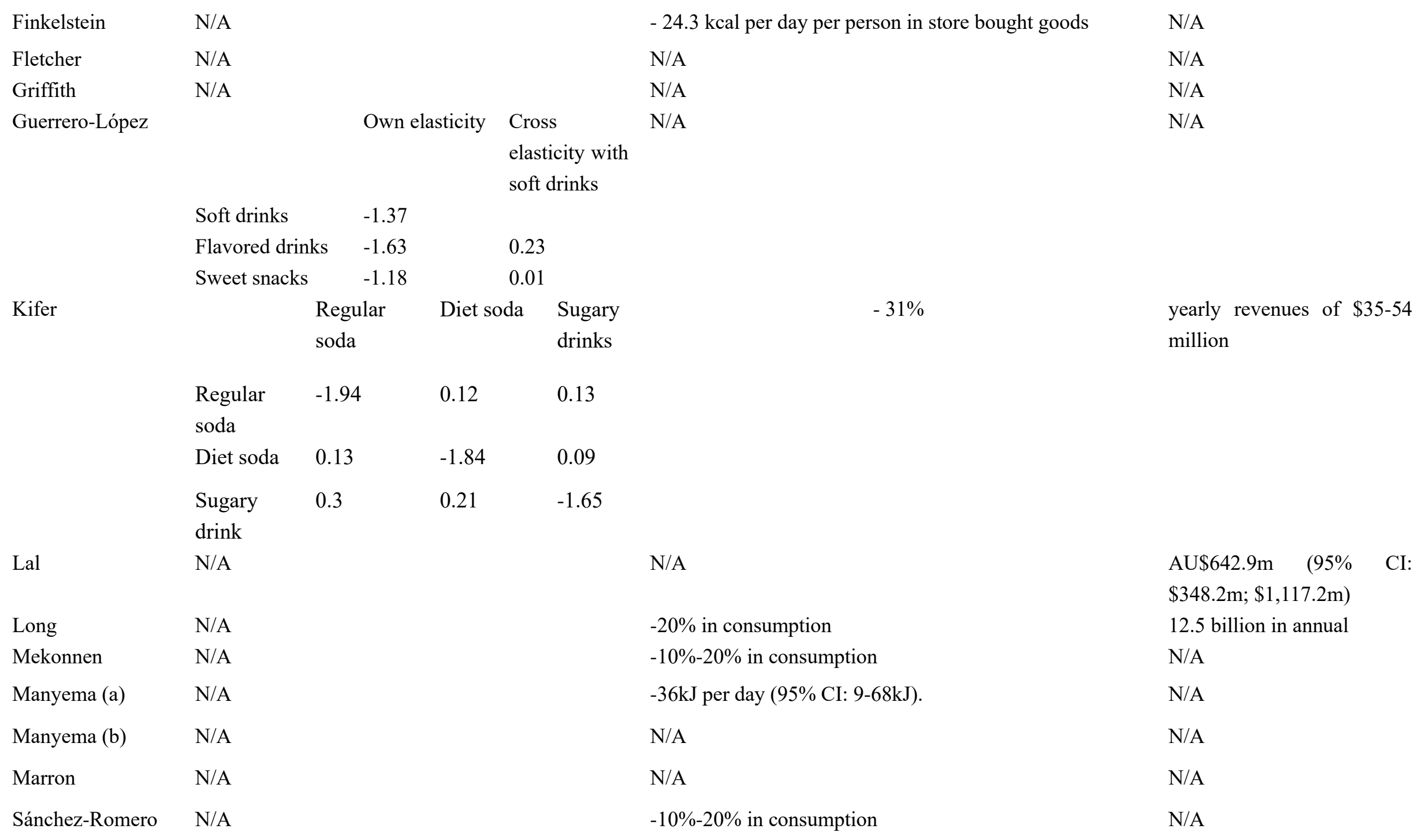




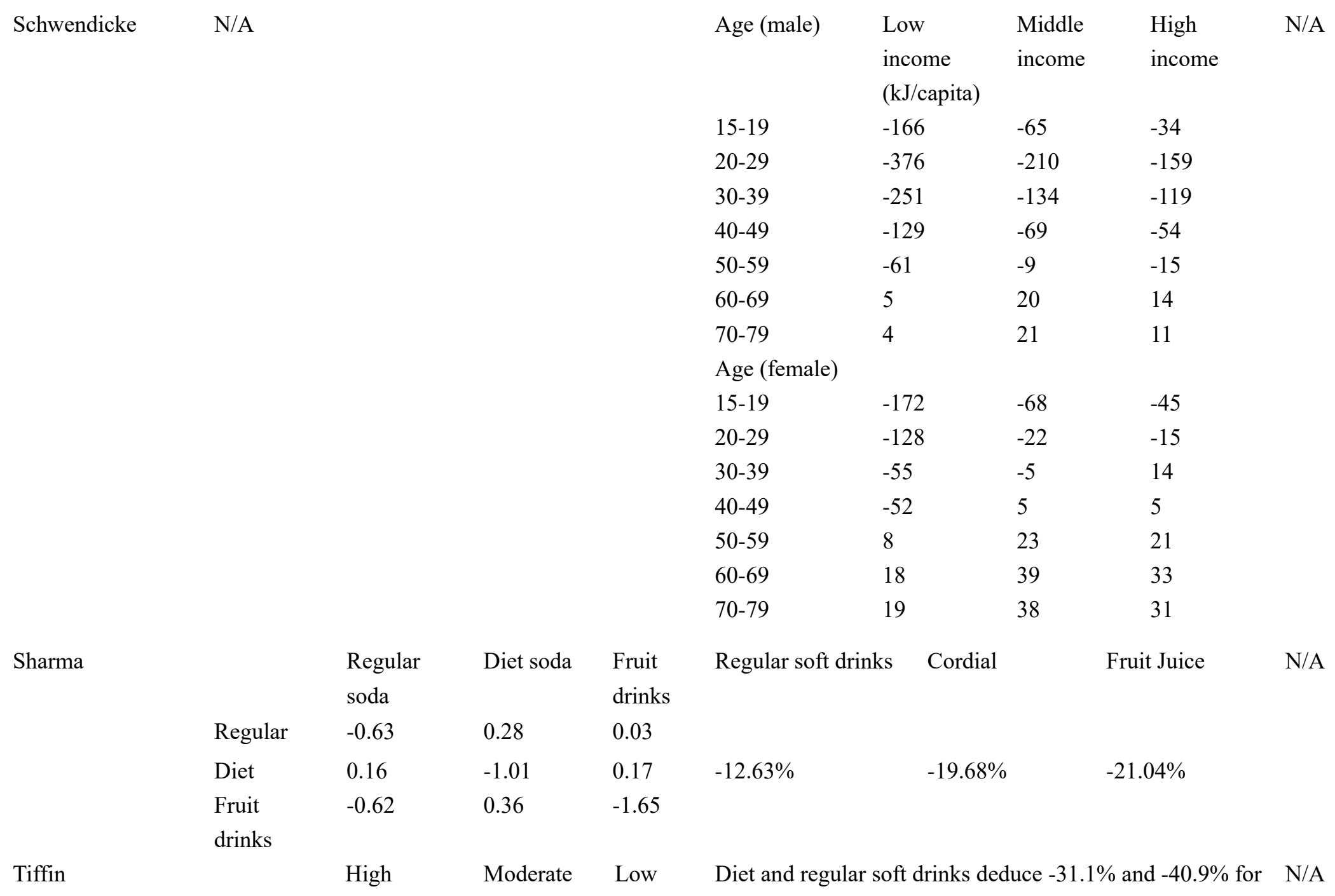


consumers

$\begin{array}{llll}\text { Diet Soda } & -1.305 & -0.784 & -0.462 \\ \text { Regular } & -1.271 & -0.759 & -0.456\end{array}$

Soda

Veerman

Wang

N/A

Own price elasticity -0.5744 , cross price elasticity 0.6302

Zhen (a)

Zhen (b)

Own elasticity -0.967 (first-stage incomplete AIDS

estimates); -1.917 (conventional DM estimates),

cross-price elasticity $-7.0 \mathrm{E}-04$ high consumers; moderate $-23.6 \%$ for regular soda
$\mathrm{N} / \mathrm{A}$

$10 \%$ sales tax

Pass-through rate

$100 \%$

$75 \%$

$50 \%$

$25 \%$

Penny per once tax

Pass-through rate

$100 \%$

$75 \%$

$50 \%$

$25 \%$

$\begin{array}{lll}\text { Low } & \text { Middle } & \text { High } \\ \text { income } & \text { income } & \text { income } \\ -3.77 \% & -3.40 \% & -3.26 \% \\ -2.69 \% & -2.62 \% & -2.37 \% \\ -2.03 \% & -1.59 \% & -1.54 \% \\ -0.99 \% & -0.79 \% & -0.69 \%\end{array}$

$-5,800 \mathrm{Kcal}$ in annual per capita beverage intake

AUD 400 million in revenue

N/A

\begin{tabular}{|c|c|c|c|c|c|c|c|}
\hline & CSD & Diet & $\begin{array}{l}\text { Sport } \\
\text { drinks }\end{array}$ & $\begin{array}{l}\text { Calories } \\
\text { kcal/day }\end{array}$ & Total & $\begin{array}{l}\text { Low } \\
\text { income }\end{array}$ & $\begin{array}{l}\text { High } \\
\text { income }\end{array}$ \\
\hline CSD & -1.035 & 0.004 & 0.15 & CSD & -7.5 & -9.2 & -6.8 \\
\hline
\end{tabular}




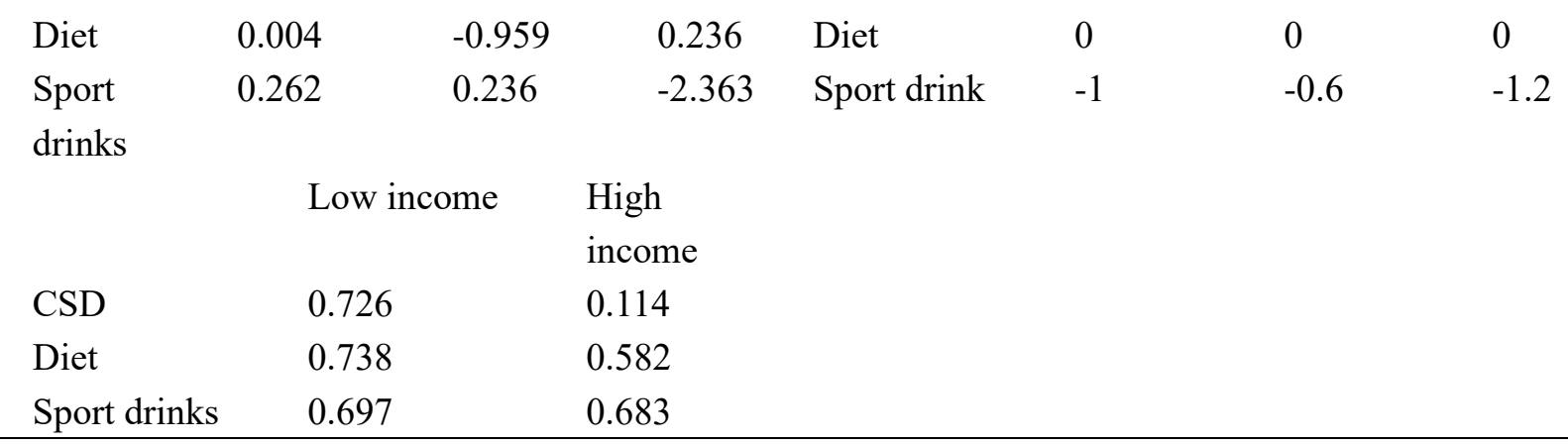


Table 2.3: Main Findings of Non-Experiment Studies (Continued)

\begin{tabular}{|c|c|c|c|}
\hline First Author & Effects on Health Risks & Change in QALYs/DALYs & Healthcare Costs \\
\hline Allcott & N/A & N/A & N/A \\
\hline \multirow{8}{*}{$\begin{array}{l}\text { Bonnet } \\
\text { Briggs (a) }\end{array}$} & $\mathrm{N} / \mathrm{A}$ & $\mathrm{N} / \mathrm{A}$ & $\mathrm{N} / \mathrm{A}$ \\
\hline & & & \\
\hline & Obesity declined by $1.3 \%$ (95\% CI $0.8 \%$ to $1.7 \%$ ) or 180000 & N/A & N/A \\
\hline & (110 000 to 247000 ) for adults; overweight decreased by $0.9 \%$ & & \\
\hline & $(0.6 \%$ to $1.1 \%)$ or 285000 (201 000 to 364000$)$. Obesity & & \\
\hline & proportions for lowest, middle, and highest income were $1.3 \%$ & & \\
\hline & $(0.3 \%$ to $2.0 \%), 0.9 \%(0.1 \%$ to $1.6 \%)$, and $2.1 \%(1.3 \%$ to & & \\
\hline & $2.9 \%)$ & & \\
\hline \multirow[t]{8}{*}{ Briggs (b) } & The proportion of the obese adults $\left(\mathrm{BMI} \geq 30 \mathrm{~kg} / \mathrm{m}^{2}\right)-1.3 \%$ or & N/A & N/A \\
\hline & 9,900 adults $(90 \% \mathrm{CI}: \quad 7,750$ to 12,940$)$, and overweight or & & \\
\hline & obese people $(\mathrm{BMI} \geq 25 \mathrm{~kg} / \mathrm{m} 2)-0.7 \%$, or 14,380 adults & & \\
\hline & $(9,790$ to 17,820$)$. Decreases in obesity: $1.2 \%$ for men and & & \\
\hline & $1.3 \%$ for women; and similar for each income group (between & & \\
\hline & $1.1 \%$ and $1.4 \%$ across income groups). $2.9 \%$ decrease in adults & & \\
\hline & aged $18-24$ years and $0.6 \%$ decline in people aged 65 years & & \\
\hline & and above. & & \\
\hline \multirow[t]{4}{*}{ Cobiac } & $\mathrm{N} / \mathrm{A}$ & SSB tax might avert $(12,000[95 \%$ UI: & The combination of tax and \\
\hline & & $2,100$ to 21,000$]$ DALYs) & subsidies might yield a net \\
\hline & & & cost-saving of $\mathrm{AU} \$ 3.4$ \\
\hline & & & billion \\
\hline Colchero (a) & $\mathrm{N} / \mathrm{A}$ & N/A & N/A \\
\hline Creedy & N/A & N/A & N/A \\
\hline Dharmasena (a) & 1.54 to $2.55 \mathrm{lb}$ per year & $\mathrm{N} / \mathrm{A}$ & $\mathrm{N} / \mathrm{A}$ \\
\hline
\end{tabular}


Dharmasena (b)

Elite

Finkelstein

Fletcher

Griffith

Guerrero-López

Kifer

Lah

Long

Lusk

Mekonnen
$-500 \mathrm{~g}$ in weight for those who consumed $151 / \mathrm{cap} / \mathrm{m} ;-800 \mathrm{~g}$ at N/A the highest consumers

-1.6 pounds on average during the first year and a cumulated N/A weight loss of 2.9 pounds in the long run.

N/A

$\mathrm{N} / \mathrm{A}$

$\mathrm{N} / \mathrm{A}$

BMI decrease of 0.97 (lowest) 1.59 (highest)

$\mathrm{N} / \mathrm{A}$

BMI by 0.16 for youth and 0.08 for adults,

The mean effect is $0.077 \mathrm{lbs} /$ year; and the high effect is 0.125 $\mathrm{lbs} /$ year; low weight effect is $0.029 \mathrm{lbs} /$ year

$1.8-3.4 \%$ decrease in diabetes, and $0.5 \%-1 \%$ in heart disease

\section{$\mathrm{N} / \mathrm{A}$}

$\mathrm{N} / \mathrm{A}$

$\mathrm{N} / \mathrm{A}$

$\mathrm{N} / \mathrm{A}$
$\mathrm{N} / \mathrm{A}$
$\$ 499 \mathrm{~m}, \quad \$ 472 \mathrm{~m}, \quad \$ 563 \mathrm{~m}$, $\$ 576 \mathrm{~m}$ in different scenarios

N/A

$\mathrm{N} / \mathrm{A}$

$\mathrm{N} / \mathrm{A}$

$\mathrm{N} / \mathrm{A}$

$\mathrm{N} / \mathrm{A}$

$5 \%$ reduction in adult BMIs would reduce healthcare costs by $\$ 29.8$ billion in 5 years, $\$ 158$ billion in 10 years, and $\$ 611.7$ billion in 20 years

$+175,300$ (95\% CI: 68,700; 277,800) Save AU\$ 1,733 million HALYs (QALYs) gains (95\% CI: \$650m; \$2,744m) over the lifetime of the population

$+871,000$ QALYs, -23.6 billion health $1^{\text {st }}$ years costs: $\$ 51 \mathrm{~m}$ related cost

N/A
$-\$ 320$ to $-\$ 620$ million

health costs 


\begin{tabular}{|c|c|c|c|}
\hline Manyema(a) & $\begin{array}{l}\text { Obesity }-3.8 \%(95 \% \text { CI: } 0.6 \%-7.1 \%) \text { in men and } 2.4 \%(95 \% \\
\text { CI: } 0.4 \%-4.4 \%) \text { in women. The number of obese adults } \\
-220,000(95 \% \text { CI: } 24,197-411,759)\end{array}$ & N/A & N/A \\
\hline Manyema(b) & $\begin{array}{l}\text { Avoid around } 72,000 \text { deaths; incident stroke cases }-85,000 \text { and } \\
\text { prevalent cases } 13,000\end{array}$ & $\begin{array}{l}550,000 \text { stroke-related } \\
\text { health-adjusted life years }\end{array}$ & $\begin{array}{l}\text { Over USD400 million in } \\
\text { health care costs over } 20 \\
\text { years (USD296-576 million) }\end{array}$ \\
\hline Marron & N/A & N/A & N/A \\
\hline Schwendicke & $\begin{array}{l}-1,028,000(-3 \% \text { relative reduction) overweight individuals } \\
\text { and } 479,000 \text { obese individuals }(-4 \%) \text {. Overweight decreased in } \\
\text { males aged } 20-29 \text { years }(408,000 \text { fewer cases } /-22 \%) \text {, the } \\
\text { same applied for obesity }(204,000 /-22 \%) \text {. }\end{array}$ & N/A & N/A \\
\hline Tiffin & N/A & N/A & N/A \\
\hline Veerman & $\begin{array}{l}\text { - } 800 \text { type } 2 \text { diabetes cases per year; }-4400 \text { heart diseases in } 25 \\
\text { years; - } 1100 \text { stroke and averted } 1606 \text { deaths. }\end{array}$ & $\begin{array}{l}+112,000 \text { health-adjusted life years for } \\
\text { men }(95 \% \text { uncertainty interval: } 73,000- \\
155,000) \text { and } 56,000 \text { ( } 95 \% \text { UI: } 36,000- \\
76,000) \text { for women }\end{array}$ & $\begin{array}{l}\text { AUD609 million (95\%UI: } \\
368 \text { million- } 870 \text { million) }\end{array}$ \\
\hline Wang & N/A & $\mathrm{N} / \mathrm{A}$ & N/A \\
\hline Zhen (a) & N/A & $\mathrm{N} / \mathrm{A}$ & N/A \\
\hline Zhen (b) & N/A & N/A & N/A \\
\hline
\end{tabular}


Table 2.4: Basic Information on Experimental Studies

\begin{tabular}{|c|c|c|c|c|c|c|}
\hline First Author & Year & Country & Tax Rate & Main Focus & Data Source / Sampling Method & Approach \\
\hline Bollard & 2016 & New Zealand & $20 \%$ & Effects on consumption & Convenient sampling & ANOVA \\
\hline Cawley & 2015 & $\begin{array}{l}\text { Berkeley, } \\
\text { USA }\end{array}$ & $\begin{array}{l}\text { one cent per } \\
\text { ounce }\end{array}$ & Pass-through rate & Convenient sampling & $\begin{array}{l}\text { Difference-in-difference; } \\
\text { linear regression }\end{array}$ \\
\hline Colchero (b) & 2016 & Mexico & $\begin{array}{l}10 \% \text { increase in } \\
\text { price }\end{array}$ & Effects on consumptions & $\begin{array}{l}\text { Nielsen Mexico's Consumer Panel } \\
\text { Services }\end{array}$ & $\begin{array}{l}\text { Difference in difference fixed } \\
\text { effect panel data linear } \\
\text { regression }\end{array}$ \\
\hline Colchero (c) & 2015 & Mexico & $\begin{array}{l}\text { a } 1 \text { peso per liter } \\
\text { excise tax }\end{array}$ & Pass-through rate & $\begin{array}{l}\text { National Institute of Statistics and } \\
\text { Geography from } 2011 \text { to } 2014 \text {; } \\
\text { National Household Income Surveys }\end{array}$ & $\begin{array}{l}\text { Pre-post quasi-experimental } \\
\text { approach using fixed effects } \\
\text { models }\end{array}$ \\
\hline Colantuoni (a) & 2015 & USA & $\begin{array}{l}5.5 \% \text { and } \\
\text { sales tax }\end{array}$ & Impacts on consumption & $\begin{array}{l}\text { IRI's sample of supermarkets; Dataset } \\
\text { B contains store sales data on } \\
\text { carbonated beverage sales and prices } \\
\text { during the 2001-2006 period }\end{array}$ & $\begin{array}{l}\text { Difference-in-difference, } \\
\text { linear regression }\end{array}$ \\
\hline Colantuoni (b) & 2014 & $\begin{array}{l}\text { Maine and } \\
\text { Ohio, USA }\end{array}$ & $\begin{array}{l}5.5 \% \text { and } 5 \% \\
\text { sales tax }\end{array}$ & Effects on obesity & $\begin{array}{l}\text { sales data collected by scanner devices } \\
\text { in Maine, Massachusetts, New York } \\
\text { and Connecticut, as well as Ohio, } \\
\text { Illinois, Michigan and Pennsylvania }\end{array}$ & $\begin{array}{l}\text { Difference-in-difference, } \\
\text { linear regression }\end{array}$ \\
\hline Cornelson (a) & 2017 & UK & $\begin{array}{l}£ 0.10 \\
\text { per-beverage }\end{array}$ & Substitutes & Itemized electronic point of sale data & $\begin{array}{l}\text { Linear regression and } \\
\text { multilevel random effects } \\
\text { models adjusting for } \\
\text { seasonality and clustering }\end{array}$ \\
\hline Cornelson (b) & 2017 & UK & $\begin{array}{l}£ 0.10 \\
\text { per-beverage }\end{array}$ & $\begin{array}{l}\text { Change in sales/elasticity; } \\
\text { substitutes }\end{array}$ & Itemized electronic point of sale data & ITS linear regression \\
\hline
\end{tabular}




\begin{tabular}{|c|c|c|c|c|c|c|}
\hline Falbe & 2015 & $\begin{array}{l}\text { Berkeley, } \\
\text { California, } \\
\text { USA }\end{array}$ & $\begin{array}{l}20 \% \text { increase in } \\
\text { price }\end{array}$ & $\begin{array}{l}\text { Price change } \\
\text { pass-through rate }\end{array}$ & Convenient sampling & $\begin{array}{l}\text { Comparison of pre vs post } \\
\text { tax price (statistical test was } \\
\text { applied but did not report } \\
\text { which method, probably } \\
\text { t-test) }\end{array}$ \\
\hline Fletcher & 2015 & USA & $\begin{array}{l}1 \% \quad-12 \% \text { of } \\
\text { price }\end{array}$ & Effects on consumptions & NHANES data. & $\begin{array}{l}\text { Difference-in-difference; } \\
\text { linear regression }\end{array}$ \\
\hline Silver & 2017 & $\begin{array}{l}\text { California, } \\
\text { USA }\end{array}$ & $\begin{array}{l}20 \% \text { increase in } \\
\text { price }\end{array}$ & $\begin{array}{l}\text { Effects on price and } \\
\text { consumptions }\end{array}$ & $\begin{array}{l}\text { Point-of-sale scanner data; a } \\
\text { representative telephone survey } \\
(17.4 \% \text { valid response) of } 957 \text { adults }\end{array}$ & t-test \\
\hline Waterlander & 2014 & Netherland & $\begin{array}{l}\text { An increase in } \\
\text { Dutch value } \\
\text { added tax from } \\
6 \% \text { to } 19 \%\end{array}$ & Substitutes & $\begin{array}{l}\text { Randomized controlled trial, sample } \\
\text { size } 91\end{array}$ & $\begin{array}{l}\text { Two sample t-test; linear } \\
\text { regression }\end{array}$ \\
\hline
\end{tabular}


Table 2.5: Main Findings of Experimental Studies

\begin{tabular}{|c|c|c|c|c|}
\hline $\begin{array}{l}\text { First } \\
\text { Author }\end{array}$ & Elasticity & Effects on substitutes & Effects on Quantity /Energy intake & Pass-through rate \\
\hline Bollard & N/A & Inapplicable & Decrease but insignificant & $\mathrm{N} / \mathrm{A}$ \\
\hline Cawley & $\mathrm{N} / \mathrm{A}$ & $\mathrm{N} / \mathrm{A}$ & Inapplicable & Over shifted \\
\hline $\begin{array}{l}\text { Colantuoni } \\
\text { (a) }\end{array}$ & $\begin{array}{l}\text { Own elasticity } \quad 0 \% \\
\text { (insignificant) }\end{array}$ & N/A & $\mathrm{N} / \mathrm{A}$ & $\mathrm{N} / \mathrm{A}$ \\
\hline $\begin{array}{l}\text { Colantuoni } \\
\text { (b) }\end{array}$ & $\begin{array}{l}\text { Didn't report (but might be } \\
\text { inferred ) }\end{array}$ & $\mathrm{N} / \mathrm{A}$ & $\begin{array}{l}\text { Did not affect the consumption } \\
\text { significantly }\end{array}$ & N/A \\
\hline $\begin{array}{l}\text { Colchero } \\
\text { (b) }\end{array}$ & Ditto & N/A & $\begin{array}{l}\text { Taxed beverages decreased by } 6 \%(-12 \\
\mathrm{mL} / \text { capita / day), and declined at an } \\
\text { increasing rate up to a } 12 \%\end{array}$ & $\mathrm{~N} / \mathrm{A}$ \\
\hline $\begin{array}{l}\text { Colchero } \\
\text { (c) }\end{array}$ & Ditto & Inapplicable & $-6.1 \%$ on average over 2014 & $\mathrm{~N} / \mathrm{A}$ \\
\hline $\begin{array}{l}\text { Cornelson } \\
\text { (a) }\end{array}$ & Ditto & $\begin{array}{l}\text { Sales per customer of children's fruit juice: }-34.7 \% \\
(-55.3 \% \text { to }-4.3 \%) \text { at } 12 \mathrm{w} \text { and } 9.9 \%(-16.8 \% \text { to } \\
-2.4 \%) \text { at } 6 \mathrm{~m} \text {. At } 6 \text { months, sales per customer of } \\
\text { fruit juice increased by } 21.8 \%(14.0 \% \text { to } 30.2 \%) \text { but } \\
\text { sales of diet cola }(-7.3 \% ;-11.7 \% \text { to }-2.8 \%) \text { and } \\
\text { bottled waters }(-6.5 \% ;-11.0 \% \text { to }-1.7 \%) \text { declined }\end{array}$ & $\begin{array}{l}\text { SSBs sold per customer decreased by } \\
11.0 \%(-17.3 \% \text { to }-4.3 \%) \text { at } 12 \text { weeks } \\
\text { and } 9.3 \%(-15.2 \% \text { to }-3.2 \%) \text { at } 6 \\
\text { months }\end{array}$ & $\mathrm{N} / \mathrm{A}$ \\
\hline $\begin{array}{l}\text { Cornelson } \\
\text { (b) }\end{array}$ & Ditto & Ditto & Ditto & $\mathrm{N} / \mathrm{A}$ \\
\hline Falbe & Ditto & N/A & $\mathrm{N} / \mathrm{A}$ & $50 \%-150 \%$ \\
\hline Fletcher & $\mathrm{N} / \mathrm{A}$ & N/A & $\begin{array}{l}\text { A } 1 \% \text { increase in the SSB taxes would } \\
\text { raise caloric intake from non-soda } \\
\text { beverages by } 7.5 \text { calories }\end{array}$ & N/A \\
\hline
\end{tabular}


Silver Did not report (but may be $3.5 \%$ increase in sales in non-taxed drinks inferred from results)

Waterlander Ditto
There were no significant effects on purchases in other beverage or snack food categories
$-9.6 \%$ in the $1^{\text {st }}$ year; self-reported N/A

mean daily SSB intake/ grams:

$-19.8 \%, \mathrm{p}=0.49$; mean per capita SSB

caloric intake: $-13.3 \%, \mathrm{p}=0.56$

-0.76 litre for sugar sweetened N/A

(unadjusted), -0.82 liter (adjusted) 


\section{Appendix II: Information for Chapter 4 - The CV study}

Table 4.1: Summary of Evidence

\begin{tabular}{|c|c|c|c|c|}
\hline First Author & $\begin{array}{l}\text { Proportion of Non-drinkers, } \\
\text { Light-to-Medium \& Heavy } \\
\text { Drinkers }\end{array}$ & $\begin{array}{l}\text { Risk to Light-to-medium } \\
\text { Drinkers Compared with } \\
\text { Non-drinkers }\end{array}$ & $\begin{array}{l}\text { Risk to Heavy Drinkers Compared with } \\
\text { Non-drinkers }\end{array}$ & Duration \\
\hline Bazzano & N/A & N/A & $18 \%$ higher risk of diabetes & 18 years \\
\hline Bernstein & $1382: 2726: 246$ & N/A & $16 \%$ higher risk of stroke & 28 years \\
\hline De Koning (a) & $\begin{array}{l}13675 \text { (never): } 16751(0.5-4 / \mathrm{w}): \\
9963(>4,5 / \mathrm{w})\end{array}$ & $\begin{array}{l}7 \%-9 \% \text { higher risk of } \\
\text { diabetes for men }\end{array}$ & $24 \%$ higher risk of developing diabetes for men & 20 years \\
\hline Eshak & N/A & $\begin{array}{l}7 \%-12 \% \text { higher risk of stroke } \\
\text { for women; } 3 \%-12 \% \text { higher } \\
\text { risk of ischemic stroke for } \\
\text { women }\end{array}$ & $\begin{array}{l}21 \% \text { higher risk of stroke for women; } 83 \% \\
\text { higher risk of ischemic stroke for women }\end{array}$ & 18 years \\
\hline Larsson & $\mathrm{N} / \mathrm{A}$ & N/A & $19 \%$ higher risk of stroke & 10.3 years \\
\hline Malik & $\mathrm{N} / \mathrm{A}$ & N/A & $26 \%$ higher risks of type 2 diabetes & $\begin{array}{l}10.96 \text { years (on } \\
\text { average) }\end{array}$ \\
\hline
\end{tabular}




\begin{tabular}{|c|c|c|c|c|}
\hline Motonen & N/A & N/A & $67 \%$ higher risk of diabetes & 12 years \\
\hline Narain & N/A & N/A & $\begin{array}{l}22 \% \text { higher risk of heart disease, } 13 \%-14 \% \\
\text { higher risk of stroke }\end{array}$ & N/A \\
\hline Palmer JR & N/A & N/A & $24 \%$ higher risk of type 2 diabetes & 10 years \\
\hline Schulze & 29203:33348:8698 & N/A & $\begin{array}{l}\text { One year water drinking (no SSBs) could reduce } \\
\text { the risk of type } 2 \text { diabetes by } 83 \%\end{array}$ & 8 years \\
\hline
\end{tabular}


Table 4.2: Binary Logistic Regression Analysis on Explain Decisions in the Pilot Survey

\begin{tabular}{|c|c|c|c|c|c|}
\hline Variable & Coefficient (SE) & Adjusted Odds Ratios / exp $\left(\beta_{j}\right)(95 \%$ CI $)$ & $Z$ value & P value & VIF \\
\hline Diabetes risk reduction & $0.005(0.003)$ & $1.005(0.999,1.011)$ & 1.751 & $0.080 *$ & 1.030 \\
\hline Stroke risk reduction & $0.015(0.003)$ & $1.015(1.010,1.020)$ & 5.466 & $<0.001 * * *$ & 1.045 \\
\hline Heart disease risk reduction & $0.014(0.008)$ & $1.014(0.999,1.029)$ & 1.809 & $0.071 *$ & 1.038 \\
\hline Income & & & & & 1.024 \\
\hline Income (90k) & $0.939(0.296)$ & $2.557(1.444,4.610)$ & 3.177 & $0.001 * *$ & \\
\hline Income $(150 \mathrm{k})$ & $1.405(0.289)$ & $4.077(2.339,7.274)$ & 4.865 & $<0.001 * * *$ & \\
\hline Income (200k) & $1.727(0.294)$ & $5.624(3.192,10.167)$ & 5.856 & $<0.001 * * *$ & \\
\hline WTP & $-0.006(0.001)$ & $0.994(0.992,0.996)$ & -6.000 & $<0.001 * * *$ & 1.088 \\
\hline Age & & & & & 1.094 \\
\hline Age (45) & $0.559(0.236)$ & $1.749(1.102,2.788)$ & 2.364 & $0.018 * *$ & \\
\hline Age (65) & $0.393(0.249)$ & $1.481(0.909,2.418)$ & 1.575 & 0.115 & \\
\hline Gender: & & & & & 1.079 \\
\hline Male & $0.807(0.218)$ & $2.241(1.468,3.453)$ & 3.705 & $<0.001 * * *$ & \\
\hline Other & $-15.560(611.700)$ & $<0.001\left(\mathrm{~N} / \mathrm{A}, 1.128 * 10^{12}\right)$ & -0.025 & 0.980 & \\
\hline Prefer not to say & $1.106(0.990)$ & $3.024(0.458,26.004)$ & 1.118 & 0.263 & \\
\hline
\end{tabular}


Asian

Pasifika

Maori

Other

Prefer not to say

Understanding

Totally agree

Somewhat agree

Neither agree nor disagree

Somewhat disagree

High Self-risk

Totally agree

Somewhat agree

Neither agree nor disagree

Somewhat disagree

Educational Level

\section{$0.347(0.284)$}

$0.959(0.531)$

$0.108(0.620)$

$-0.011(1.033)$

$0.042(0.528)$

$0.510(0.426)$

$0.154(0.418)$

$-0.471(0.510)$

$-0.127(0.553)$

$1.995(0.543)$

$1.300(0.337)$

$1.084(0.307)$

$0.479(0.267)$
$1.415(0.810,2.474)$

$1.114(0.383,3.102)$

$2.608(0.787,9.195)$

$0.989(0.108,7.370)$

$1.043(0.370,2.968)$

$1.666(0.733,3.920)$

$1.166(0.520,2.697)$

$0.625(0.229,1.706)$

$0.881(0.298,2.627)$

$7.351(2.605,22.140)$

$3.670(1.907,7.176)$

$2.955(1.626,5.434)$

$1.614(0.961,2.744)$

$\begin{array}{cc}1.219 & 0.223 \\ 0.203 & 0.839 \\ 1.546 & 0.122 \\ -0.011 & 0.992 \\ 0.080 & 0.936\end{array}$

1.078

$\begin{array}{ll}1.199 & 0.230 \\ 0.368 & 0.713 \\ -0.923 & 0.356 \\ -0.230 & 0.818\end{array}$

$3.675<0.001^{* * *}$

$3.853<0.001 * * *$

$3.526<0.001^{* * *}$

$1.792 \quad 0.073$ 


\begin{tabular}{|c|c|c|c|c|}
\hline Certificate or Diploma & $-0.354(0.362)$ & $7.019(0.341,1.416)$ & -0.977 & 0.328 \\
\hline Bachelor's & $-0.119(0.259)$ & $8.882(0.533,1.472)$ & -0.458 & 0.647 \\
\hline Postgraduate & $-0.919(0.500)$ & $3.989(0.147,1.047)$ & -1.840 & $0.073^{*}$ \\
\hline Intercept & $-3.721(0.571)$ & $0.0242(0.008,0.072)$ & -6.492 & $<0.001 * * *$ \\
\hline
\end{tabular}

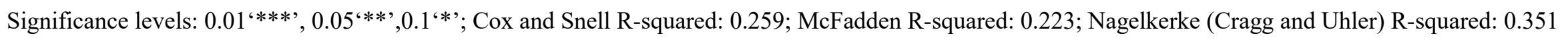




\section{Questionnaire of the Pilot Survey Using Students}

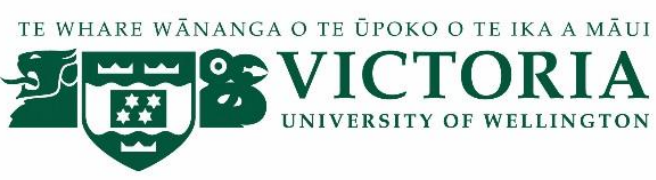

\section{An Estimation of People's Willingness-to-pay for Health Risks Reductions}

\section{INFORMATION FOR PARTICIPANTS for Anonymous Surveys}

You are invited to take part in this research. Please read this information before deciding whether to take part. If you decide to participate, thank you. If you decide not to participate, thank you for considering this request. And your course grade will not be affected by whether you participate or not.

\section{Who am I?}

My name is Ningxin Ding, and I am a master student in the Victoria University of Wellington. This research project is towards my thesis.

\section{What is the aim of the project?}

The objective of the project is to elicit willingness to pay for the kind of health improvements that have been suggested for a soda tax. This data will be used to infer the benefits of such a tax and will be used in the optimal tax formula which is to be derived separately. This research has been approved by the Victoria University of Wellington Human Ethics Committee.

\section{How can you help?}

You have been invited to participate because you are a person who has adequate life experience and critical thinking skills. If you agree to take part in, this survey will ask you questions about your willingness-to-pay for three disease risks reductions. This survey will take you around 10 to 15 minutes to finish. 


\section{What will happen to the information you give?}

This research is anonymous. This means that nobody, including the researchers will be aware of your identity. By answering it, you are giving consent for us to use your responses in this research. Your answers will remain completely anonymous and unidentifiable. Once you submit the survey, it will be impossible to retract your answer. Please do not include any personal identifiable information in your responses.

\section{What will the project produce?}

The information from my research will be used in my master thesis.

\section{If you have any questions or problems, who can you contact?}

If you have any questions, either now or in the future, please feel free to contact me:

\section{Student:}

Name: Ningxin Ding

University email address:

dingning1@myvuw.ac.nz

\section{Supervisor:}

Name: Paul Calcott

Role: senior lecturer

School: Economics and Finance

\section{Human Ethics Committee information}

If you have any concerns about the ethical conduct of the research, you may contact the Victoria University HEC Convenor: Associate Professor Susan Corbett. Email susan.corbett@vuw.ac.nz or telephone +64-4-463 5480. 
Q1-6 Suppose at age X, you have a job with an annual income of $\mathrm{Y}$ after tax; you are allowed to borrow some money from your bank, and the amount you can borrow from the bank depend entirely on your income. Today your GP offers you a pill (one shot) which will reduce the risk of developing diabetes, stroke, and heart disease by $\mathrm{a} \%, \mathrm{~b} \%$ and $\mathrm{c} \%$ respectively in the next ten years. Unfortunately, this pill is neither paid for by the NZ healthcare system nor covered by any health insurance. Please note that if you do become sick, your health problems would not only reduce your quality of life, but also cause income loss and other costs. Please answer the question below on a basis of the scenario described above and your personal and family history of disease:

Would you be willing to pay $\mathrm{Z}$ (one shot) for the pill?

Yes $\square \quad$ No $\square$

Q7 Would you say that you perfectly understand the scenario described above, and your answers to Q1-6 were made on the basis of a comprehensive consideration of health risks, financial conditions, costs of the pill, and personal health status etc.?
Totally disagree $\square$
Somewhat disagree
Neither agree nor disagree $\square$
Somewhat agree
Totally agree $\square$

Prefer not to say

Q8 Your gender: Female $\square \quad$ Male $\square \quad$ Other $\square \quad$ Prefer not to say $\square$ 
Q9 Your ethnicity:

NZ European $\square \quad$ Māori $\square \quad$ Pasifika $\square \quad$ Asian $\square \quad$ African $\square \quad$ Latino American $\square \quad$ Prefer not to say $\square \quad$ Other, please specify

Q10 What is your highest educational level you have completed?

Secondary education or below $\square \quad$ Certificate or Diploma $\square \quad$ Bachelor's degree $\square \quad$ Honours or Postgraduate $\square \quad$ Masters $\square \quad$ Doctoral $\square$

Prefer not to say

Q11 Would you say that you currently are or will be a person with relatively high risk of developing the three diseases mentioned in the scenario?

(e.g. if you have family history of the three diseases, smoke, or seldom do exercise, then you would probably be a person with high risk)

Totally disagree $\square \quad$ Somewhat disagree $\square \quad$ Neither agree nor disagree $\square \quad$ Somewhat agree $\square \quad$ Totally agree $\square$

Prefer not to say

Q12 What is your frequency of consuming sugar-sweetened soft drinks or artificial-sweetened drinks (eg. Coca-Cola, red-bull, fruit juice etc.) per month on average? (Please fill in the blank with a number, and decimal numbers are OK )

a) I consume tins sugar-sweetened soft drinks or artificial-sweetened drinks per month on average. 
b) I consume $600 \mathrm{ml}$-size bottles sugar-sweetened soft drinks or artificial-sweetened drinks per month on average.

c) I consume 1.5L-size bottles sugar-sweetened soft drinks or artificial-sweetened drinks per month on average.

d) I consume 3L-size bottles sugar-sweetened soft drinks or artificial-sweetened drinks per month on average.

CV Questionnaire of pilot survey

Suppose at age X, you have a job with an annual income 200k after tax, and your bank account balance depends entirely on your income. Today your GP offers you a pill (one shot) which will reduce the risk of developing diabetes, stroke, and heart disease by $83 \%$, $83 \%$ and $35 \%$ respectively in the next ten years. Unfortunately, this pill is neither paid for by the NZ healthcare system nor covered by any health insurance. Further assume that at age X, you have not been diagnosed as a diabetes patient, but you have family history of these three diseases, or other diseases which could significantly threaten your quality of life. Please note that if you do become sick, your health problems would not only reduce your quality of life, but also cause income loss and other costs. Based on the scenario described above, please state how much you would be willing to pay for the pill at the three hypothetical ages below.

Q1 At age 20, I would be willing to pay $\mathrm{NZ} \$$ for the pill. 
Q3 At age 60, I would be willing to pay NZ\$ for the pill.

Q4 If you gave a zero willingness-to-pay in any one of the questions 1-3, please briefly explain why you were not willing to pay anything.

I was not willing to pay anything because

Q5 Your gender: Female $\square \quad$ Male $\square \quad$ Other $\square \quad$ Prefer not to say $\square$

Q6 Your ethnicity:

NZ European $\square \quad$ Māori $\square \quad$ Pasifika $\square \quad$ East Asian $\square \quad$ Southeast Asian $\square \quad$ Indian $\square \quad$ Prefer not to say $\square \quad$ Other, please
specify

Q7 How many times do you consume sugar-sweetened soft beverages or artificially sweetened beverages (e.g., Coca-Cola, Red Bull, fruit juice etc.) per week?

I consume sugar-sweetened soft drinks and artificially sweetened drinks times per week. 


\section{D-optimal Design for the Pilot Survey}

Diabetes Stroke IHD Income WTP Age

10

45

53

83

154

177

198

205

241

257

272

279

328

342

399

427

434

443

462

484

600

611

621

643

652

688

728

741

757

806

839

861

884

906

922

969

995

1006

1049

1058

1128

1155

$\begin{array}{llllll}7 & 3 & 19 & 30 & 1 & 25\end{array}$

$\begin{array}{llllll}83 & 83 & 19 & 90 & 1 & 25\end{array}$

$\begin{array}{llllll}26 & 83 & 35 & 90 & 1 & 25\end{array}$

$\begin{array}{llllll}26 & 3 & 4 & 200 & 1 & 25\end{array}$

$\begin{array}{llllll}7 & 3 & 35 & 90 & 3 & 25\end{array}$

$\begin{array}{llllll}83 & 21 & 19 & 150 & 3 & 25\end{array}$

$\begin{array}{llllll}83 & 83 & 4 & 200 & 3 & 25\end{array}$

$\begin{array}{llllll}7 & 83 & 19 & 200 & 3 & 25\end{array}$

$\begin{array}{llllll}7 & 83 & 35 & 30 & 5 & 25\end{array}$

$\begin{array}{llllll}26 & 21 & 19 & 90 & 5 & 25\end{array}$

$\begin{array}{llllll}26 & 3 & 4 & 150 & 5 & 25\end{array}$

$\begin{array}{llllll}83 & 83 & 4 & 150 & 5 & 25\end{array}$

$\begin{array}{llllll}7 & 21 & 4 & 30 & 10 & 25\end{array}$

$\begin{array}{llllll}83 & 83 & 19 & 30 & 10 & 25\end{array}$

$\begin{array}{llllll}83 & 3 & 35 & 150 & 10 & 25\end{array}$

$\begin{array}{llllll}7 & 21 & 35 & 200 & 10 & 25\end{array}$

$\begin{array}{llllll}26 & 3 & 4 & 30 & 30 & 25\end{array}$

$\begin{array}{llllll}26 & 3 & 19 & 30 & 30 & 25\end{array}$

$\begin{array}{llllll}83 & 3 & 4 & 90 & 30 & 25\end{array}$

$\begin{array}{llllll}7 & 83 & 35 & 90 & 30 & 25\end{array}$

$\begin{array}{llllll}83 & 21 & 4 & 150 & 80 & 25\end{array}$

$\begin{array}{llllll}26 & 83 & 19 & 150 & 80 & 25\end{array}$

$\begin{array}{llllll}83 & 83 & 35 & 150 & 80 & 25\end{array}$

$\begin{array}{llllll}7 & 21 & 35 & 200 & 80 & 25\end{array}$

$\begin{array}{lllll}7 & 21 & 4 & 30180 & 25\end{array}$

$\begin{array}{lllll}7 & 21 & 19 & 90180 & 25\end{array}$

$\begin{array}{llllll}26 & 83 & 35 & 150180 & 25\end{array}$

$\begin{array}{lllll}83 & 3 & 19 & 200180 & 25\end{array}$

$\begin{array}{lllll}7 & 3 & 4 & 30382 & 25\end{array}$

$\begin{array}{lllll}26 & 21 & 35 & 90382 & 25\end{array}$

$\begin{array}{lllll}26 & 3 & 4 & 200382 & 25\end{array}$

$\begin{array}{lllll}83 & 21 & 35 & 200382 & 25\end{array}$

$\begin{array}{llllll}26 & 3 & 35 & 30 & 1 & 45\end{array}$

$\begin{array}{llllll}83 & 21 & 19 & 90 & 1 & 45\end{array}$

$\begin{array}{llllll}7 & 21 & 4 & 150 & 1 & 45\end{array}$

$\begin{array}{llllll}83 & 21 & 35 & 200 & 1 & 45\end{array}$

$\begin{array}{llllll}26 & 21 & 35 & 30 & 3 & 45\end{array}$

$\begin{array}{llllll}7 & 83 & 4 & 90 & 3 & 45\end{array}$

$\begin{array}{llllll}26 & 21 & 35 & 150 & 3 & 45\end{array}$

$\begin{array}{llllll}26 & 21 & 4 & 200 & 3 & 45\end{array}$

$\begin{array}{llllll}83 & 3 & 35 & 90 & 5 & 45\end{array}$

$\begin{array}{llllll}83 & 3 & 35 & 150 & 5 & 45\end{array}$ 


$\begin{array}{rrrrrrr}1175 & 26 & 21 & 19 & 200 & 5 & 45 \\ 1177 & 7 & 83 & 19 & 200 & 5 & 45 \\ 1197 & 83 & 83 & 4 & 30 & 10 & 45 \\ 1232 & 26 & 83 & 19 & 90 & 10 & 45 \\ 1247 & 26 & 21 & 4 & 150 & 10 & 45 \\ 1252 & 7 & 3 & 19 & 150 & 10 & 45 \\ 1302 & 83 & 21 & 4 & 30 & 30 & 45 \\ 1363 & 7 & 21 & 19 & 150 & 30 & 45 \\ 1383 & 83 & 21 & 4 & 200 & 30 & 45 \\ 1403 & 26 & 83 & 35 & 200 & 30 & 45 \\ 1415 & 26 & 3 & 19 & 30 & 80 & 45 \\ 1425 & 83 & 3 & 35 & 30 & 80 & 45 \\ 1443 & 83 & 3 & 19 & 90 & 80 & 45 \\ 1492 & 7 & 83 & 4 & 200 & 80 & 45 \\ 1539 & 83 & 83 & 35 & 30 & 180 & 45 \\ 1548 & 83 & 83 & 4 & 90 & 180 & 45 \\ 1576 & 7 & 3 & 19 & 150 & 180 & 45 \\ 1600 & 7 & 83 & 4 & 200 & 180 & 45 \\ 1630 & 7 & 3 & 19 & 30382 & 45 \\ 1664 & 26 & 83 & 19 & 90 & 382 & 45 \\ 1666 & 7 & 3 & 35 & 90382 & 45 \\ 1681 & 7 & 83 & 4 & 150382 & 45 \\ 1755 & 83 & 83 & 35 & 30 & 1 & 65 \\ 1783 & 7 & 3 & 4 & 150 & 1 & 65 \\ 1790 & 26 & 83 & 4 & 150 & 1 & 65 \\ 1822 & 7 & 21 & 19 & 200 & 1 & 65 \\ 1848 & 83 & 3 & 19 & 30 & 3 & 65 \\ 1862 & 26 & 83 & 35 & 30 & 3 & 65 \\ 1866 & 83 & 3 & 4 & 90 & 3 & 65 \\ 1900 & 7 & 3 & 19 & 150 & 3 & 65 \\ 1949 & 26 & 21 & 4 & 30 & 5 & 65 \\ 1960 & 7 & 83 & 19 & 30 & 5 & 65 \\ 1977 & 83 & 21 & 4 & 90 & 5 & 65 \\ 2044 & 7 & 3 & 35 & 200 & 5 & 65 \\ 2093 & 26 & 21 & 19 & 90 & 10 & 65 \\ 2098 & 7 & 3 & 35 & 90 & 10 & 65 \\ 2142 & 83 & 83 & 4 & 200 & 10 & 65 \\ 2153 & 26 & 3 & 35 & 200 & 10 & 65 \\ 2212 & 7 & 83 & 35 & 90 & 30 & 65 \\ 2228 & 26 & 21 & 19 & 150 & 30 & 65 \\ 2239 & 7 & 83 & 35 & 150 & 30 & 65 \\ 2253 & 83 & 3 & 19 & 200 & 30 & 65 \\ 2290 & 7 & 21 & 35 & 30 & 80 & 65 \\ 2297 & 26 & 3 & 4 & 90 & 80 & 65\end{array}$




$\begin{array}{rrrrrrr}2299 & 7 & 21 & 4 & 90 & 80 & 65 \\ 2366 & 26 & 83 & 19 & 200 & 80 & 65 \\ 2391 & 83 & 21 & 19 & 30180 & 65 \\ 2405 & 26 & 3 & 4 & 90180 & 65 \\ 2453 & 26 & 21 & 35 & 150180 & 65 \\ 2477 & 26 & 3 & 35 & 200180 & 65 \\ 2492 & 26 & 83 & 4 & 30382 & 65 \\ 2556 & 83 & 83 & 19 & 150382 & 65 \\ 2562 & 83 & 21 & 35 & 150382 & 65 \\ 2580 & 83 & 21 & 19 & 200382 & 65\end{array}$


Table 4.4: Binary Logistic Regression Analysis on Explain Decisions in the Survey on Facebook

\begin{tabular}{|c|c|c|c|c|c|}
\hline Variable & Coefficient (SE) & $\begin{array}{l}\text { Adjusted Odds Ratios / } \\
\operatorname{Exp}\left(\beta_{\mathrm{j}}\right)(95 \% \text { Confidence Interval })\end{array}$ & $Z$ value & $P$ value & VIF \\
\hline Diabetes risk reduction & $0.006(0.304)$ & $1.006(1.004,1.008)$ & 6.384 & $<0.001 * * *$ & 1.025 \\
\hline Stroke risk reduction & $0.012(0.001)$ & $1.012(1.010,1.014)$ & 13.926 & $<0.001^{* * *}$ & 1.008 \\
\hline Heart disease risk reduction & $0.018(0.001)$ & $1.018(1.014,1.023)$ & 7.502 & $<0.001 * * *$ & 1.025 \\
\hline $25 \mathrm{k}$ to $70 \mathrm{k}$ & $-0.208(0.101)$ & $0.756(0.620,0.922)$ & -2.756 & $0.006^{* *}$ & \\
\hline $70 \mathrm{k}$ to $120 \mathrm{k}$ & $0.477(0.118)$ & $1.611(1.278,2.032)$ & 4.033 & $<0.001 * * *$ & \\
\hline Above $120 \mathrm{k}$ & $-0.095(0.161)$ & $0.909(0.662,1.246)$ & -0.596 & $<0.555$ & \\
\hline Prefer not to say & $-0.733(0.148)$ & $0.480(0.358,0.641)$ & -4.942 & $<0.001 * * *$ & \\
\hline 25 to 45 & $-0.552(0.119)$ & $0.576(0.456,0.728)$ & -4.628 & $<0.001 * * *$ & \\
\hline 45 to 65 & $-0.432(0.132)$ & $0.649(0.501,0.841)$ & -3.279 & $0.001 * *$ & \\
\hline Above 65 & $0.604(0.191)$ & $1.830(1.257,2.661)$ & 3.159 & $0.002 * *$ & \\
\hline Prefer not to say & - $1.998(0.970)$ & $0.136(0.011,0.664)$ & -2.060 & $0.039^{*}$ & \\
\hline
\end{tabular}


Gender

Male

Race

\section{Asian}

Pasifika

Maori

Other

Understanding

Totally agree

Somewhat agree

Neither agree nor disagree

\section{Self-risk}

Totally agree

Somewhat agree

Neither agree nor disagree

Somewhat disagree

Prefer not to say

$$
0.103(0.0734)
$$

$1.108(0.959,1.279)$

$0.749(0.113)$

$0.327(0.197)$

- $0.236(0.116)$

$-0.011(0.150)$

$0.192(0.152)$

$0.661(0.155)$

$-0.320(0.206)$

0.682 (0.129)

$0.441(0.127)$

$0.545(0.141)$

$0.122(0.126)$

0.834 (0.356)
$0.532(0.394,0.711)$

$2.114(1.694,2.639)$
$1.386(0.936,2.030)$
$0.790(0.629,0.990)$
$0.532(0.394,0.711)$

$1.211(0.900,1.635)$

$1.936(1.433,2.626)$

$0.726(0.485,1.086)$

$1.977(1.539,2.548)$

1.554 (1.213, 1.998)

1.725 (1.309, 2.276)

$1.130(0.883,1.449)$

2.302 (1.141, 4.612)
1.222

$1.397 \quad 0.163$

1.181

$\begin{array}{ll}6.619 & <0.001 * * * \\ 1.656 & 0.098 \\ -2.032 & 0.042 * \\ -4.200 & <0.001 * * *\end{array}$

1.275

$\begin{array}{ll}1.260 & 0.208 \\ 4.275 & <0.001 * * * \\ -1.559 & 0.119\end{array}$

$5.298<0.001 * * *$

$3.467<0.001 * * *$

$3.861<0.001 * * *$

$0.996 \quad 0.334$

$2.346 \quad 0.019 *$ 
Educational Level

Certificate or Diploma

Bachelor's

Postgraduate

Prefer not to say

Good Knowledge of the Diseases

Totally agree

Somewhat agree

Neither agree nor disagree

More Government Spending

\section{Totally agree}

Somewhat agree

Neither agree nor disagree

Somewhat disagree

Intercept

$$
\begin{array}{ll}
-0.041(0.109) & 0.959(0.775,1.189) \\
0.496(0.105) & 1.642(1.337,2.019) \\
0.061(0.121) & 1.063(0.839,1.348) \\
-0.444(0.202) & 0.642(0.430,0.949)
\end{array}
$$$$
-0.265(0.169)
$$$$
-0.565(0.165)
$$$$
0.266(0.183)
$$$$
0.767(0.552,1.071)
$$$$
0.568(0.412,0.786)
$$$$
1.304(0.913,1.869)
$$$$
-0.379
$$$$
4.720<0.001 * * *
$$$$
0.505 \quad 0.614
$$$$
\text { - } 2.1960 .028^{*}
$$

$-1.568 \quad 0.117$$$
-3.431 \quad 0.001 * * *
$$$$
1.455 \quad 0.146
$$

$\begin{array}{ll}0.071(0.225) & 1.074(0.697,1.684) \\ -0.151(0.228) & 0.860(0.554,1.358) \\ 0.079(0.231) & 1.082(0.694,1.716) \\ -0.605(0.273) & 0.546(0.321,0.936) \\ -1.743(0.304) & 0.175(0.096,0.316)\end{array}$

$\begin{array}{ll}0.316 & 0.752 \\ -0.661 & 0.509 \\ 0.343 & 0.732 \\ -2.214 & 0.027^{*} \\ -5.725 & <0.001^{* * *}\end{array}$

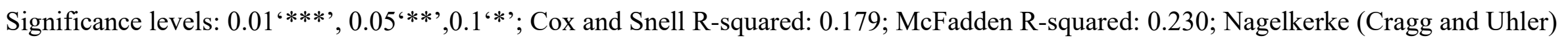
R-squared: 0.300; Weights ( 0.627 for females, and 2.824 for males) were used to adjust the distortion in gender distribution in the sample. 


\section{D-optimal design of the survey on Facebook}

Diabetes Stroke IHD WTP

$\begin{array}{lllll}1 & 26 & 3 & 4 & 1\end{array}$

$\begin{array}{lllll}2 & 26 & 21 & 4 & 1\end{array}$

$\begin{array}{lllll}3 & 83 & 21 & 4 & 1\end{array}$

$\begin{array}{lllll}4 & 83 & 21 & 19 & 1\end{array}$

$\begin{array}{lllll}5 & 7 & 83 & 19 & 1\end{array}$

$\begin{array}{lllll}6 & 26 & 83 & 19 & 1\end{array}$

$\begin{array}{lllll}7 & 7 & 3 & 35 & 1\end{array}$

$\begin{array}{lllll}8 & 83 & 3 & 35 & 1\end{array}$

$\begin{array}{lllll}9 & 7 & 83 & 35 & 1\end{array}$

$\begin{array}{lllll}10 & 7 & 3 & 4 & 3\end{array}$

$\begin{array}{lllll}11 & 7 & 21 & 4 & 3\end{array}$

$\begin{array}{lllll}12 & 83 & 83 & 4 & 3\end{array}$

$\begin{array}{lllll}13 & 26 & 3 & 19 & 3\end{array}$

$\begin{array}{lllll}14 & 26 & 21 & 19 & 3\end{array}$

$\begin{array}{lllll}15 & 83 & 21 & 19 & 3\end{array}$

$\begin{array}{lllll}16 & 7 & 3 & 35 & 3\end{array}$

$\begin{array}{lllll}17 & 26 & 83 & 35 & 3\end{array}$

$\begin{array}{lllll}18 & 83 & 83 & 35 & 3\end{array}$

$\begin{array}{lllll}19 & 26 & 3 & 4 & 5\end{array}$

$\begin{array}{lllll}20 & 83 & 21 & 4 & 5\end{array}$

$\begin{array}{lllll}21 & 7 & 83 & 4 & 5\end{array}$

$\begin{array}{lllll}22 & 7 & 3 & 19 & 5\end{array}$

$\begin{array}{lllll}23 & 7 & 21 & 19 & 5\end{array}$

$\begin{array}{lllll}24 & 26 & 21 & 19 & 5\end{array}$

$\begin{array}{lllll}25 & 83 & 3 & 35 & 5\end{array}$

$\begin{array}{lllll}26 & 26 & 83 & 35 & 5\end{array}$

$\begin{array}{lllll}27 & 83 & 83 & 35 & 5\end{array}$

$\begin{array}{lllll}28 & 7 & 3 & 4 & 10\end{array}$

$\begin{array}{lllll}29 & 7 & 21 & 4 & 10\end{array}$

$\begin{array}{lllll}30 & 83 & 83 & 4 & 10\end{array}$

$\begin{array}{lllll}31 & 26 & 3 & 19 & 10\end{array}$

$\begin{array}{lllll}32 & 26 & 21 & 19 & 10\end{array}$

$\begin{array}{lllll}33 & 7 & 83 & 19 & 10\end{array}$

$\begin{array}{lllll}34 & 26 & 3 & 35 & 10\end{array}$

$\begin{array}{lllll}35 & 83 & 21 & 35 & 10\end{array}$

$\begin{array}{lllll}36 & 83 & 83 & 35 & 10\end{array}$

$\begin{array}{lllll}37 & 26 & 21 & 4 & 30\end{array}$

$\begin{array}{lllll}38 & 26 & 83 & 4 & 30\end{array}$

$\begin{array}{lllll}39 & 83 & 83 & 4 & 30\end{array}$

$\begin{array}{lllll}40 & 7 & 3 & 19 & 30\end{array}$

$\begin{array}{lllll}41 & 83 & 3 & 19 & 30\end{array}$

$\begin{array}{lllll}42 & 7 & 83 & 19 & 30\end{array}$ 


$\begin{array}{rrrrr}43 & 7 & 3 & 35 & 30 \\ 44 & 26 & 21 & 35 & 30 \\ 45 & 83 & 21 & 35 & 30 \\ 46 & 26 & 3 & 4 & 80 \\ 47 & 83 & 21 & 4 & 80 \\ 48 & 26 & 83 & 4 & 80 \\ 49 & 83 & 3 & 19 & 80 \\ 50 & 7 & 83 & 19 & 80 \\ 51 & 83 & 83 & 19 & 80 \\ 52 & 7 & 3 & 35 & 80 \\ 53 & 7 & 21 & 35 & 80 \\ 54 & 26 & 21 & 35 & 80 \\ 55 & 83 & 3 & 4 & 180 \\ 56 & 7 & 83 & 4 & 180 \\ 57 & 83 & 83 & 4 & 180 \\ 58 & 26 & 3 & 19 & 180 \\ 59 & 83 & 3 & 19 & 180 \\ 60 & 7 & 21 & 19 & 180 \\ 61 & 7 & 21 & 35 & 180 \\ 62 & 26 & 21 & 35 & 180 \\ 63 & 26 & 83 & 35 & 180 \\ 64 & 7 & 3 & 4 & 382 \\ 65 & 26 & 3 & 4 & 382 \\ 66 & 7 & 21 & 4 & 382 \\ 67 & 83 & 21 & 19 & 382 \\ 68 & 26 & 83 & 19 & 382 \\ 69 & 83 & 83 & 19 & 382 \\ 70 & 83 & 3 & 35 & 382 \\ 71 & 26 & 21 & 35 & 382 \\ 72 & 7 & 83 & 35 & 382\end{array}$




\section{Questionnaire on Facebook on Facebook}

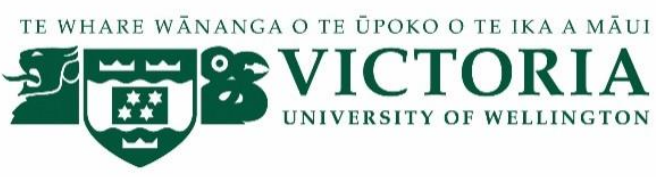

\section{An Estimation of People's Willingness-to-pay for Health Risks \\ Reductions}

\section{INFORMATION FOR PARTICIPANTS for Anonymous Surveys}

You are invited to take part in this research. Please read this information before deciding whether or not to take part. If you decide to participate, thank you. If you decide not to participate, thank you for considering this request.

\section{Who am I?}

My name is Ningxin Ding, and I am a PhD candidate at the Victoria University of Wellington. This research project is towards my thesis.

\section{What is the aim of the project?}

A sugar tax is a widely used instrument to reduce sugar consumption and thus improve people's health. Like other taxes it works by raising the price of things (food and drinks) that have sugar in them. The reasonable expectation is that people will consume less of things that have higher prices.

Economic theory suggests that the optimal tax rate is related to how much people are willing to pay for an improvement in their health. Unlike prices of things in the market (grocery story, dairies, etc.) this willingness to pay for health improvements is not directly observable, and a survey of the type you are being asked to participate in is one way to obtain this information. The data you and other respondents provide will be used to determine the size of an optimal sugar tax, and its likely effect on sugar consumption in New Zealand.

This research has been approved by the Victoria University of Wellington Human Ethics Committee (0000025927). 


\section{How can you help?}

If you agree to take part, this survey will ask you some basic questions about your background, dispositions, and willingness to pay for diabetes, stroke, and heart disease risk reductions. This survey will take around 10 to 15 minutes to complete.

\section{What will happen to the information you give?}

This research is anonymous. This means that nobody, including the researchers will be aware of your identity. By answering it, you are giving consent for us to use your responses in this research. Your answers will remain completely confidential. You are free to stop answering questions at any point of your choosing, and your incomplete questionnaire will not be used in the research. Once you submit the survey, it will be impossible to retract your answers. Please do not include any personal identifiable information in your responses.

At the end of the survey, you can choose to enter a draw for one \$200 Countdown voucher. A link will be provided at the end of the questionnaire. It will take you to a separate site for entering your email address. After all questionnaires have been completed and the survey is officially closed, the email addresses of those who have chosen to enter the draw will be used for selecting one winner. Only this (email address) information will be used to conduct the draw. Once the winner has been notified the email addresses will be deleted. This ensures that your answers to the survey questions will not be linked to your email address and are thus entirely anonymous. It also means that your survey responses will have no bearing on the chances of your winning the draw.

\section{What will the project produce?}

The information from my research will be used in my $\mathrm{PhD}$ thesis.

\section{If you have any questions or problems, who can you contact?}

If you have any questions, either now or in the future, please feel free to contact me:

\section{Student:}

Name: Ningxin Ding

University email address:

dingning1@ myvuw.ac.nz

\section{Supervisor:}

Name: Jaikishan Desai

Role: Senior Lecturer

School: Government 


\section{Human Ethics Committee information}

If you have any concerns about the ethical conduct of the research, you may contact the Victoria University HEC Convenor: $\mathrm{Dr}$ Judith Loveridge. Email: judith.loveridge @vuw.ac.nz or telephone +64-4-463 6028. 


\title{
WILLINGNESS TO PAY SURVEY
}

\author{
FOR A PILL THAT REDUCES THE RISK OF
}

DIABETES, STROKE, AND HEART DISEASE

NOTE TO REVIEWERS: This is an annotated questionnaire in draft form. The annotations appear in italics below each question. The questionnaire has been designed for face-to-face interviews, and upon ethics approval will be re-formatted for an online survey. It will be pilot tested and modified on the basis of feedback; our expectation is that the modifications will be primarily in the wording and ordering of questions.

A document containing an explanation of the study design and the analysis plan is included at the very end of the questionnaire

My name is Ningxin Ding, and I am a post-graduate student at Victoria University of Wellington. As part of my thesis I am doing a survey to find out how much people are willing to pay for reduction in health risks associated with consumption of food and drinks that have sugar in them.

Are you willing to participate in this survey?

It will take approximately 10 minutes to finish.

At the end of the survey you can enter a Draw for two Countdown grocery vouchers worth $\$ 50$ each.

Scientific evidence suggests that reduction in sugar consumption can improve people's health by reducing the risk of diabetes, stroke, and heart disease. One way to get people to reduce sugar consumption is to a put a tax on things (food and drinks) that have sugar in them. The expectation is that a tax will increase the price of food and drinks that have sugar in them, and higher prices will make people buy less of these things. What we do not know is how much tax is required to reduce sugar consumption and how much people are willing to pay for a reduction in health risks. 
The information you and others provide in this survey is anonymous and confidential meaning nobody, including I, will know your identity and so your information will remain completely confidential. You are free to stop answering questions at any point of your choosing, and your incomplete questionnaire will not be used in the research. Once you submit the survey, it will be impossible to retract your answers.

This research has been approved by the Victoria University of Wellington's Human Ethics Committee (0000025927). If you agree to take part, this survey will ask you some basic questions about your background, and your willingness to pay for diabetes, stroke, and heart disease risk reductions.

At the end of the survey, you can choose to enter a draw for two $\$ 50$ Countdown vouchers that will be drawn randomly from all survey participants.

If you have any questions, either now or in the future, please feel free to contact me:

Name: Ningxin Ding

University email address: dingning1@myvuw.ac.nz

If you are willing to participate, click the Next button.

If you do not wish to continue, click the Back button to exit survey.

Now I would like to ask you some questions about your willingness to pay for a single pill offered by your GP that reduces your risk of developing diabetes, stroke, and heart disease by different amounts over the next 10 years. These are hypothetical scenarios.

In answering the questions, please keep in mind that this is a hypothetical scenario and there is no such a pill. Please note that if you do become sick from these diseases, your health problems might not only reduce your quality of life but could also cause income loss and other costs. Please answer the questions below on basis of the scenario described below, bearing in mind your personal circumstances such as income, age, sex, and your personal and family history of disease.

An example of WTP questions:

\begin{tabular}{|l|l|l|l|l|}
\hline No. & Scenario & Response \\
\hline & $\begin{array}{l}\text { Would you be willing to pay \$1000 at one go for the pill if it } \\
\text { were to reduce risk of developing diabetes, stroke, and heart } \\
\text { disease by [amounts below] respectively in the next ten years? }\end{array}$ & \\
\hline $\begin{array}{l}\text { Risk Reduction } \\
\text { for... }\end{array}$ & Diabetes & Stroke & Heart Disease & \\
\hline & $\mathbf{2 6 \%}$ & $\mathbf{3 \%}$ & $\mathbf{4 \%}$ & Yes/No \\
\hline
\end{tabular}


Explanation: These are the core WTP questions. The risk reduction values have been generated by XXXXXXX and are the same in each questionnaire. The WTP amount $\$ X X X X$ ranges from $\$ 1,000$ to $\$ 328,000$ and will be randomly assigned to questionnaires so different people will see different amounts (See WTP Study Design Explanation attachment for explanation on calculation of these amounts). These randomly allocated amounts will provide a range of WTP responses for the same health risk reduction. Responses to these questions make up the dependent variable in the binary logistic regression.

Q10 Would you say that you understand the scenarios described above, and your answers to Q1-9 were made on the basis of a full consideration of your circumstances (for example, health risks, financial conditions, costs of the pill, and personal health status)?

Totally disagree

Somewhat disagree

Neither agree nor disagree

Somewhat agree

Totally agree

Prefer not to say

Explanation: This question has been included to select meaningful responses, these being those where respondents say they understand the scenarios. Insufficiently considered responses (of YES or NO) are of limited use in the estimation of average WTP estimation. While there is no way to guarantee that the response to this question is a perfect indicator of meaningful responses to questions 1 to 9, (internal) consistency checks and the response to this question will help in selecting questionnaires that have received careful consideration by respondents.

Q11 Your gender:

Female

Male

Other

Prefer not to say

Q12 Your age:

Below 25

Between 25 and $45 \square$

Between 45 and 65

Above 65 
Prefer not to say

Q13 Your annual income after tax:

Below 25k

Between 25k and 70k

Between 70 and 120k

Above 120k

Prefer not to say

Q14 Your ethnicity:

NZ European

Māori

Pasifika

Asian

Other

Q15. What is the highest educational level you have completed?
Secondary or below
Certificate or Diploma
Bachelor's degree
Postgraduate
Prefer not to say

Explanation: Questions 11 to 15 are put into the questionnaire to collect basic demographic information. These variables are considered likely to be relevant and important, based on the findings of previous CV studies (Smith \& Sach, 2009; Zagefka, Noor, \& Brown, 2013; Howley, 2016). These will be included as explanatory variables in the binary logistic regression.

Q16. Would you say that you have a good knowledge of the three diseases mentioned in the scenario?

Totally disagree

Somewhat disagree

Neither agree nor disagree

Somewhat agree 
Totally agree

Prefer not to say

Explanation: This question is put into the questionnaire because people who have a good knowledge of diseases are thought to be more likely to be willing to pay for risk reduction (give a YES answer to the WTP questions). For example, some people may think that diabetes is not a big issue because harm of diabetes cannot be seen immediately. These people might be more likely to not be willing to pay for risk reduction. People who know that complications of diabetes can cause serious health problems are more likely to be willing to pay for risk reduction (give a YES answer to WTP questions).

Q17 Would you say that you currently are or will be a person with relatively high risk of developing the three diseases mentioned in the scenario? (e.g., if you have family history of the three diseases, smoke, or seldom do exercise, then you would probably be a person with high risk)

Totally disagree

Somewhat disagree

Neither agree nor disagree

Somewhat agree

Totally agree

Prefer not to say

Explanation: This question is put into the questionnaire because people who have a family history, smoke, or leave a sedentary life style might be more willing to pay for risk reduction (give a YES answer to WTP questions).

Q18 Do you believe government should spend more money on diabetic, stroke, and heart diseases patients? (e.g., subsidy for drugs, rehabilitation, \& prevention etc.)

Totally disagree

Somewhat disagree

Neither agree nor disagree

Somewhat agree

Totally agree

Prefer not to say 
Explanation: This question is put into the questionnaire to test strategic bias. That is, people who believe that once the result is published, government is more likely to spend more money on diabetic, stroke, and heart diseases patients if a high WTP value is seen. Therefore, people who believe that government should spend more money on diabetic, stroke, and heart diseases patients are more likely to give a YES answer. 


\section{Facebook Communities Surveyed}

Samples were drawn from the Facebook users in the following community groups:

1. Wairarapa Trade \& Exchange

2. Buy / Sell / Swap - Hutt Valley

3. Lower Hutt/Wellington-Buy, Sell or Swap Anything

4. Lower Hutt Buy / Sell / Trade

5. Titahi Bay Buy / Sell / Trade

6. Wellington Buy Sell Swap Anything New Zealand

7. Paraparaumu Buy / Sell / Trade Ltd

8. Kapiti Coast, Buy, Sell, Trade, Free, Swaps

9. Wellington Capital Deals! (Buy, Sell, Trade)

10. Upper Hutt Buy Sell Give Away

11. Sell Anything Wellington

12. For Sale, Free \& Wanted in Wellington NZ

13. Hutt Valley Quick Sales

14. Wellington Region Buy / Sell

15. Porirua Buy Sell Trade No Rules

16. Wainuiomats Selling or Swapping or Free

17. Buy/Sell/Trade Wellington - NZ

18. Buy, Sell, Trade, Wellington Wide

19. Buy \& Sell- Johnsonville / Surrounding Suburbs!

20. Carterton Buy \& Sell/Pay It Forward

21. Done Deal (Wellington \& Surrounding Areas)

22. Online Garage Sale South Wairarapa

23. Upper Hutt Buy, Sell Swap ONLY

24. Buy \$ Sell Wellington

25. Wairarapa Trading Buy $\sim$ Sell $\sim$ Swap

26. Island Bay Buy Sell Swap Free, Buy, Sell

27. Swap Lower Hutt

28. Eastbourn Local Services

29. Mount Wellington Buy Sell Swap Anything

30. Friends of Whitby NZ

31. Kilbirnie and Its Neighbours

32. Feathersrton, New Zealand Business Group

33. Porirua Online Garage Sale

34. Wairarapa Anything for Sale

35. Wellington Sell, Swap, Buy, Giveaway,

36. Buy Sell Anything in Wellington

37. Churton Park Neighbours

38. Johnsonville / Surrounding Surburbs, Community and Local Business

39. Ngaio Buy, Sell or Free

40. Owhiro Bay News \& Views 
41. Newlands / Paparangi / Woodridge Community

42. Wainuimata - Pay It Forward.

43. Buy / Sell / Swap - Upper Hutt

44. Ngaio, Crofton Downs, Khandallah, Broadmeadows Community Group

45. Khandallah - For Sale, Free, or Wanted

46. Greytown KZN Trade Zone

47. Buy / Sell / Trade Wellington / Upper / Lower Hutt / Porirua

48. Wellington (buy / sell / trade)

49. Wellington Region Buy / Sell / Trade

50. Kapiti Coast - Buy, Trade, \& Sell, Buy

51. Naenae Swap, Sell, Buy or Free, Naenae Locals Only

52. Buy, Sell or Swap Wellington

53. Items for Sale in Wellington NZ 


\section{Appendix III: Information for IHD and Stroke Costs}

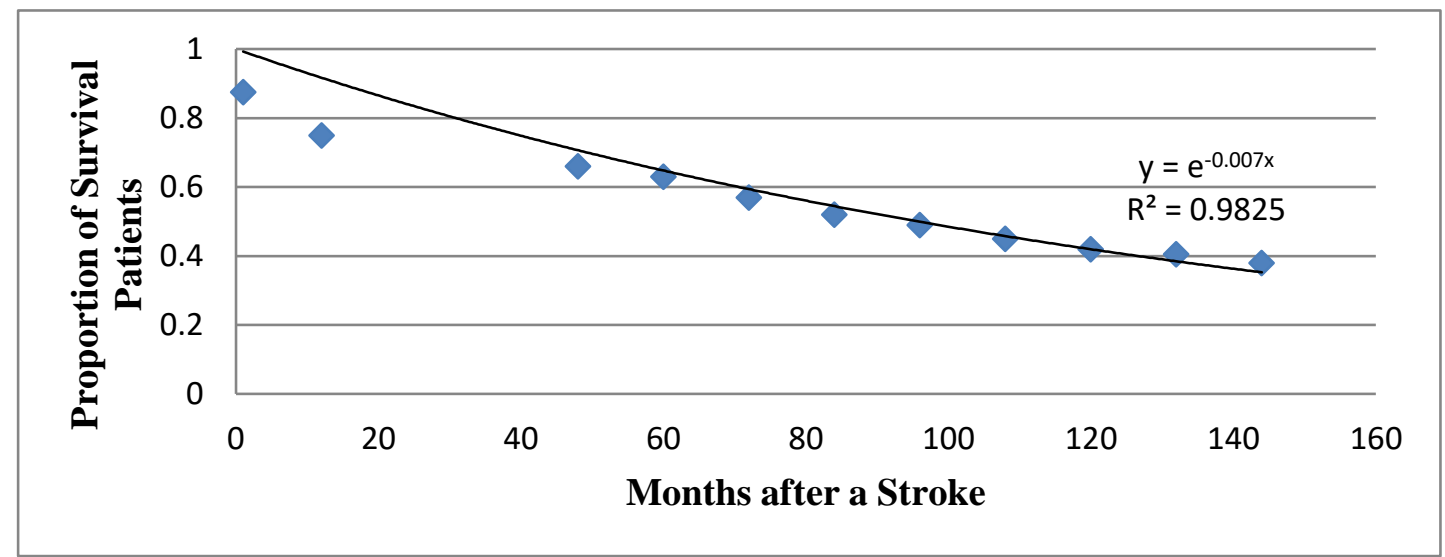

Figure 5.5: Proportion of Survival Patients after a Stroke (Cerebral Haemorrhage Only)

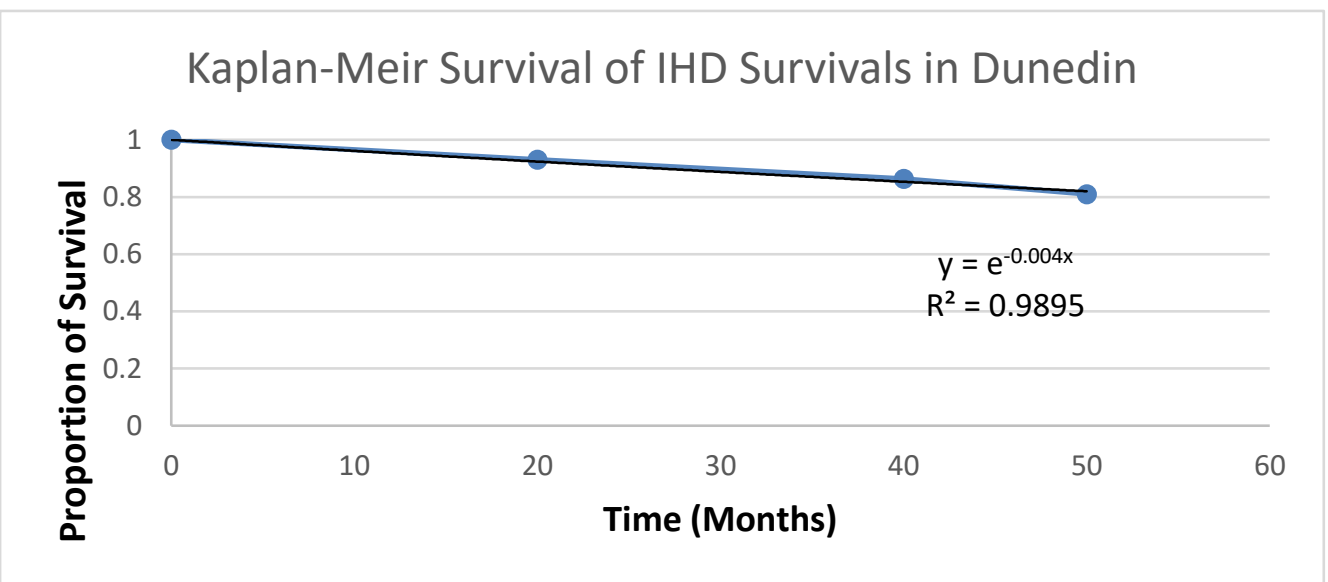

Figure 5.6: Proportion of Survival Patients after IHD Discharge in Dunedin

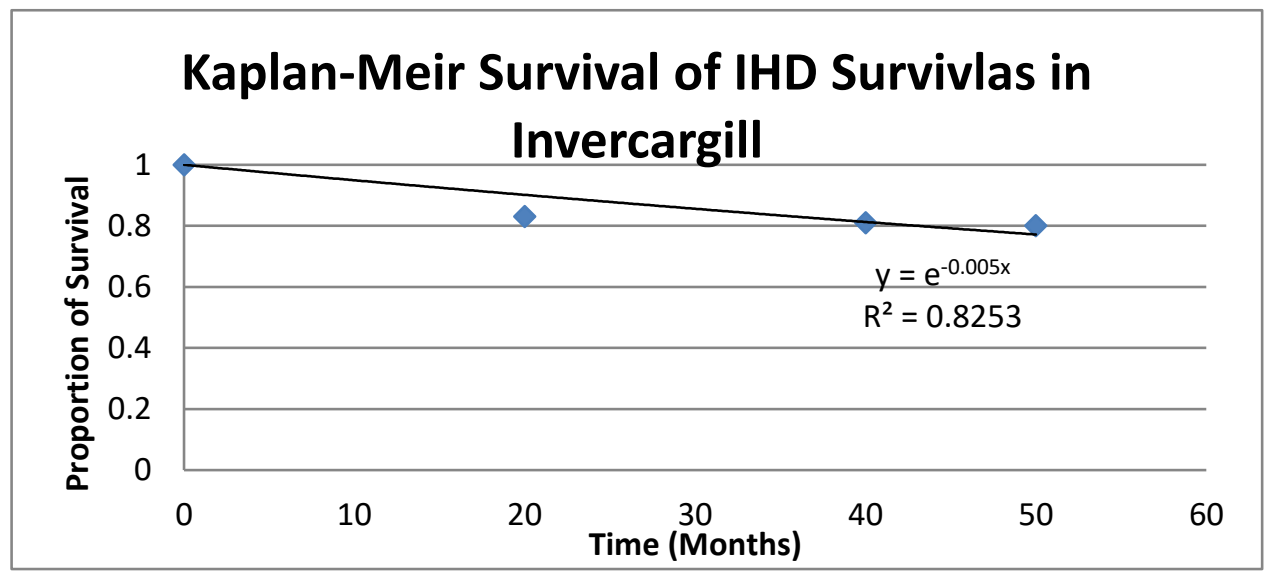

Figure 5.7: Proportion of Survival Patients after IHD Discharge in Invercargill 


\section{Suggested Long Term Interventions in the Guideline}

(1) Suggested long term interventions after a stroke

In the New Zealand primary care handbook (New Zealand Primary Care Handbook 2012, 2012), the suggested interventions following a stroke were the following:

- Long-term antiplatelet and anticoagulation interventions should be applied to all ischemic stroke survivors.

- Low dose aspirin and clopidogrel are recommended for those stroke survivors who (1) have acute coronary disease or a recent coronary stent, (2) have had a neurological stent procedure

- All patients after stroke, whether normotensive or hypertensive, should receive blood pressure lowering therapy for secondary prevention

- Benefits have been found from the use of an ACE inhibitor after a stroke

- Different interventions have been found to be effective in lowering blood pressure, except for beta-blockers.

(2) The suggested interventions following an IHD

- Oral aspirin (75to $150 \mathrm{mg} / \mathrm{d}$ ) should be given routinely and perpetual.

- Warfarin should be used for patients with atrial fibrillation, a left ventricular aneurysm, or thrombus demonstrated in the left ventricle at the infarction site.

- Beta-blockers (e.g. metoprolol or propranolol) and an ACE should be considered for every survivor unless contraindicated.

- A statin (20-40 mg daily) should be started early, and continued.

\section{(3) Suggested interventions following a MI}

- All people post-MI or angina should be on aspirin, a statin, and a beta-blocker and considered for an ACE inhibitor

- Oral Aspirin (75-150) mg should be given routinely and be perpetual.

- Clopidogrel (75 mg/day) is a feasible alternative to aspirin for patients with contraindications to, or who are intolerant to aspirin.

- Warfarin should be used for high-risk MI patients with atrial fibrillation, a left ventricular aneurysm, or thrombus demonstrated in the left ventricle at the infarction site.

- Beta-blockers (e.g. metoprolol or propranolol) should be considered for every MI survivor unless contraindicated.

- An ACE inhibitor should be considered for every MI survivor. The treatment should be started early and continued.

- A statin (20-40 mg daily) should be started early after a MI, and continued. 
Table 5.1: Costs of Stroke: The Most Serious Situation

\begin{tabular}{|c|c|c|c|c|c|c|c|c|c|c|c|}
\hline Event & \multicolumn{2}{|c|}{ Year at the event } & \multirow[b]{2}{*}{ Source } & \multirow[b]{2}{*}{$\begin{array}{l}\text { DRG } \\
\text { Unit } \\
\text { price }\end{array}$} & \multirow[b]{2}{*}{ Source } & \multirow[t]{2}{*}{ Subsequent years } & \multirow[b]{2}{*}{ Unit price } & \multirow[b]{2}{*}{ Source } & \multirow[b]{2}{*}{ Dosage } & \multirow[b]{2}{*}{ Source } & \multirow[b]{2}{*}{$\begin{array}{l}\text { Total } \\
\text { annual } \\
\text { costs } \\
\end{array}$} \\
\hline Event & $\begin{array}{l}\text { DRG Units } \\
\text { (Fatal) }\end{array}$ & $\begin{array}{l}\text { DRG Units } \\
\text { (Non-Fatal) }\end{array}$ & & & & & & & & & \\
\hline Stroke & 0.6747 & 2.1016 & $\begin{array}{l}2018 \\
\text { DRG }\end{array}$ & $\begin{array}{l}\$ 5,06 \\
8.12\end{array}$ & $\mathrm{MoH}$ & $\begin{array}{l}\text { Cilazapril with } \\
\text { hydrochlotothiazide }\end{array}$ & $\begin{array}{l}5 \mathrm{mg} \text { per tab; } \\
\$ 10.8 \text { per } 100 \text { tab }\end{array}$ & $\begin{array}{l}\text { PHARMAC } \\
\text { online } \\
\text { schedule }\end{array}$ & $5 \mathrm{mg} / \mathrm{d}$ & $\begin{array}{l}\text { Primary } \\
\text { care } \\
\text { handbook; } \\
\text { Medsafe }\end{array}$ & $\$ 39.42$ \\
\hline Rehabilitation & & $\$ 6,715.15$ & Ditto & Ditto & Ditto & & & & & & \\
\hline & & & & & & Statin & $\begin{array}{l}40 \mathrm{mg} \text { per tab: } \\
\$ 15.93 \text { per } 500\end{array}$ & Ditto & $40 \mathrm{mg} / \mathrm{d}$ & Ditto & $\$ 11.63$ \\
\hline & & & & & & Aspirin & $\begin{array}{l}100 \mathrm{mg} \text { per tab; } \\
\$ 10.8 \text { per } 990\end{array}$ & Ditto & $100 \mathrm{mg} / \mathrm{d}$ & Ditto & $\$ 3.98$ \\
\hline & & & & & & Clopidogrel & $\begin{array}{l}75 \mathrm{mg} \text { per tab; } 5.44 \\
\text { per } 84 \text { tab }\end{array}$ & Ditto & $75 \mathrm{mg} / \mathrm{d}$ & Ditto & $\$ 23.64$ \\
\hline & & & & & & Neurologist & $\$ 424.86$ per time & PU & $\begin{array}{l}4 \text { visits } \\
\text { per year }\end{array}$ & $\begin{array}{l}\text { Expert } \\
\text { opinion }\end{array}$ & $\begin{array}{l}\$ 1,699.4 \\
4\end{array}$ \\
\hline & & & & & & Full blood count & $\$ 8.55$ per test & $\mathrm{PU}$ & 4 tests & Ditto & $\$ 34.71$ \\
\hline & & & & & & $\begin{array}{l}\text { Rehabilitation } \\
\text { (same day) }\end{array}$ & $\$ 212.90$ per time & PU & $\begin{array}{l}\text { Once } \\
\text { every } 2 \\
\text { weeks }\end{array}$ & & $\begin{array}{l}\$ 5,633.8 \\
7\end{array}$ \\
\hline & & & & & & $\begin{array}{l}\text { Residential care } \\
\text { cost }\end{array}$ & $\begin{array}{l}\$ 8,119,632 / 299 * 1 \\
015 * 0.2=\$ 5,512.66\end{array}$ & & & $\begin{array}{l}\text { Social } \\
\text { economic } \\
\text { cost of } \\
\text { stroke in } \\
\mathrm{NZ}\end{array}$ & $\begin{array}{l}\$ 5,512.6 \\
6\end{array}$ \\
\hline DRG total cost & $\$ 3,470.02$ & $\$ 17,526.08$ & & & & None-DRG cost & $\$ 34,946.13$ & & & & \\
\hline $\begin{array}{l}\text { Total cost at } \\
\text { the event }\end{array}$ & $\$ 3,470.02$ & $\$ 52,472.21$ & & & & & & & & & \\
\hline $\begin{array}{l}\text { Annual cost } \\
\text { after year1 }\end{array}$ & $\$ 34,946.13$ & & & & & Informal Care costs & $\begin{array}{l}\$ 41,742,398 / 1,927 \\
* 1.015=\$ 21,986.78\end{array}$ & & & Ditto & $\begin{array}{l}\$ 21,986 . \\
78\end{array}$ \\
\hline
\end{tabular}


Table 5.2 Costs of Stroke: the Most Conservative Situation

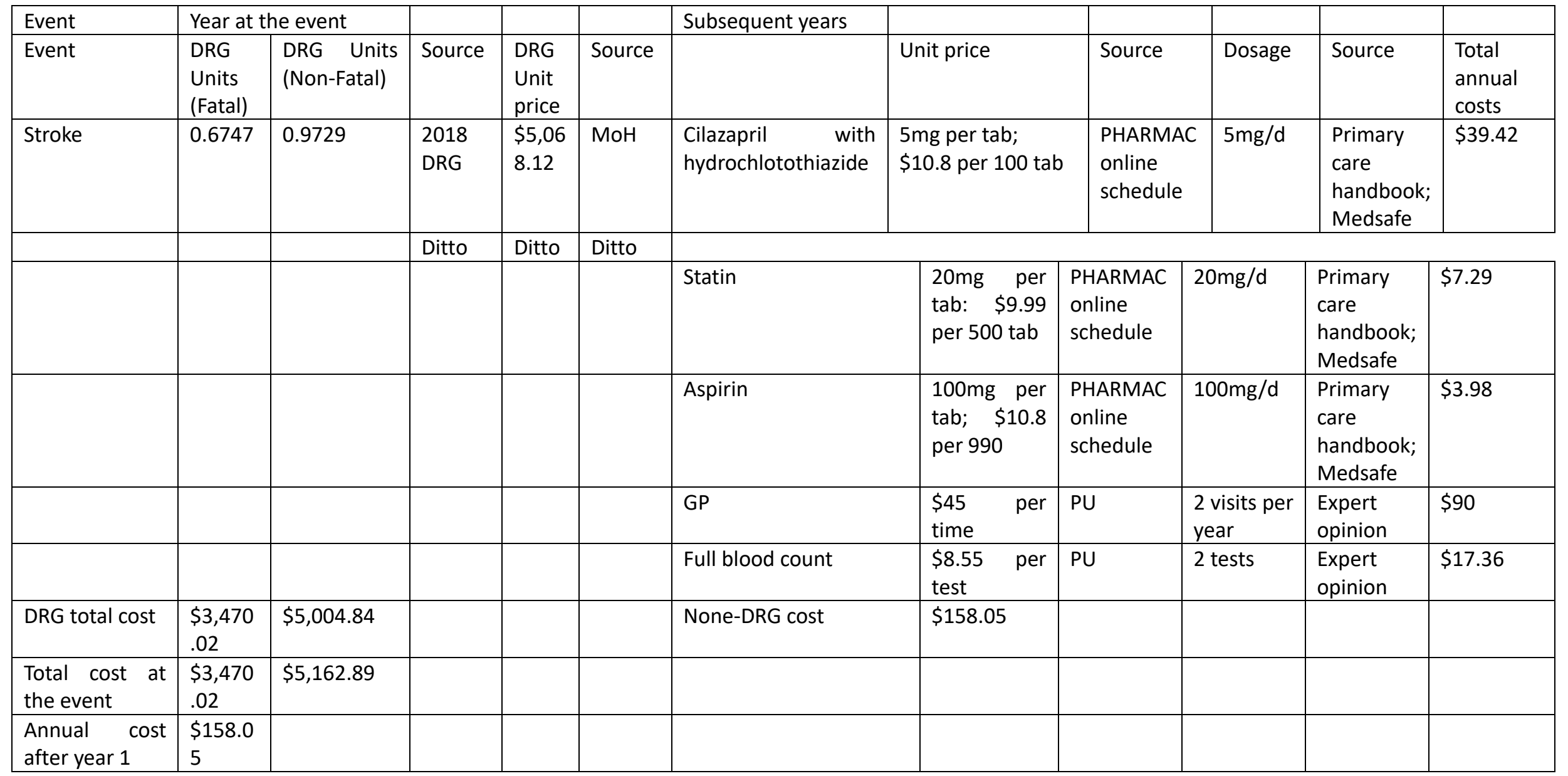


Table 5.3 Costs of Stroke: Base Case

\begin{tabular}{|c|c|c|c|c|c|c|c|c|c|c|c|}
\hline Event & \multicolumn{2}{|c|}{ Year at the event } & \multirow[b]{2}{*}{ Source } & \multirow[b]{2}{*}{$\begin{array}{l}\text { DRG Unit } \\
\text { price }\end{array}$} & \multirow[b]{2}{*}{ Source } & \multirow[t]{2}{*}{ Subsequent years } & \multirow[b]{2}{*}{ Unit price } & \multirow[b]{2}{*}{ Source } & \multirow[b]{2}{*}{ dosage } & \multirow[b]{2}{*}{ Source } & \multirow[b]{2}{*}{$\begin{array}{l}\text { Total } \\
\text { annual } \\
\text { costs }\end{array}$} \\
\hline Event & $\begin{array}{l}\text { DRG Units } \\
\text { (Fatal) }\end{array}$ & $\begin{array}{l}\text { DRG } \\
\text { Units } \\
\text { (Non- } \\
\text { Fatal) }\end{array}$ & & & & & & & & & \\
\hline \multirow[t]{6}{*}{ Stroke } & 0.6747 & $\begin{array}{l}1.2916 \\
1.4554\end{array}$ & $\begin{array}{l}2018 \\
D R G\end{array}$ & $\$ 5,068.12$ & $\mathrm{MoH}$ & $\begin{array}{l}\text { Cilazapril+ } \\
\text { hydrochlotothiazide }\end{array}$ & $\begin{array}{l}\text { 5mg per tab; } \\
\$ 10.8 \text { per } 100 \\
\text { tab }\end{array}$ & $\begin{array}{l}\text { PHARMAC } \\
\text { online } \\
\text { schedule }\end{array}$ & $5 \mathrm{mg} / \mathrm{d}$ & $\begin{array}{l}\text { Primary } \\
\text { care } \\
\text { handbook; } \\
\text { Medsafe }\end{array}$ & $\$ 39.42$ \\
\hline & & & Ditto & Ditto & Ditto & Clopidogrel & $\begin{array}{l}75 \mathrm{mg} \text { per tab; } \\
5.44 \text { per } 84 \text { tab }\end{array}$ & Ditto & $\begin{array}{l}75 \mathrm{mg} / \\
\mathrm{d}\end{array}$ & Ditto & $\$ 23.64$ \\
\hline & & & & & & Statin & $\begin{array}{lll}20 m g & \text { per } & \text { tab: } \\
\$ 9.99 & \text { per } & 500 \\
\text { tab } & & \\
\end{array}$ & Ditto & $\begin{array}{l}30 \mathrm{mg} / \\
\text { d }\end{array}$ & Ditto & $\$ 10.94$ \\
\hline & & & & & & Aspirin & $\begin{array}{l}100 \mathrm{mg} \text { per tab; } \\
\$ 10.8 \text { per } 990\end{array}$ & Ditto & $\begin{array}{l}100 \mathrm{mg} \\
/ \mathrm{d}\end{array}$ & Ditto & $\$ 3.98$ \\
\hline & & & & & & Neurologist & $\begin{array}{l}\text { \$424.86 per } \\
\text { time }\end{array}$ & $\mathrm{PU}$ & $\begin{array}{l}2 \text { visits } \\
\text { per/y }\end{array}$ & $\begin{array}{l}\text { Expert } \\
\text { opinion }\end{array}$ & $\$ 862.47$ \\
\hline & & & & & & Full blood count & $\$ 8.55$ per test & $\mathrm{PU}$ & 2 tests & Ditto & $\$ 17.36$ \\
\hline \multirow[t]{2}{*}{$\begin{array}{ll}\text { DRG total } \\
\text { cost }\end{array}$} & $\$ 3,470.02$ & $\$ 14,314$ & & & & None-DRG cost & $\$ 16,612.2$ & & & & \\
\hline & & & & & & $\begin{array}{l}\text { Residential care } \\
\text { cost }\end{array}$ & $\begin{array}{l}\$ 8,119,632 / 299 \\
* 1.015 * 0.1=2,7 \\
56.33\end{array}$ & & & $\begin{array}{l}\text { Social and } \\
\text { economic } \\
\text { costs of } \\
\text { stoke in } \\
\text { New } \\
\text { Zealand }\end{array}$ & $\$ 2,756.33$ \\
\hline $\begin{array}{l}\text { Total cost at } \\
\text { the event }\end{array}$ & $\$ 3,470.02$ & $\begin{array}{l}\$ 30.926 . \\
2\end{array}$ & & & & Informal Care costs & $\begin{array}{l}\text { women median } \\
2018: \$ 23.75 / \mathrm{h}\end{array}$ & Stats NZ & $\begin{array}{l}427.48 \\
\text { hours/y }\end{array}$ & $\begin{array}{l}\text { Claesson } \\
\text { et al. }\end{array}$ & $\$ 10,304.94$ \\
\hline $\begin{array}{l}\text { Annual cost } \\
\text { after } 1\end{array}$ & $\$ 14,019.08$ & & & & & $\begin{array}{l}\text { Rehabilitation } \quad\left(1^{\text {st }}\right. \\
\text { year only })\end{array}$ & $\begin{array}{l}\$ 212.90 \quad \text { per } \\
\text { time }\end{array}$ & PU & $\begin{array}{l}1 \\
\text { /month }\end{array}$ & & $\$ 2,593.12$ \\
\hline
\end{tabular}


Table 5.4 Costs of IHD: The Most Serious Situation

\begin{tabular}{|c|c|c|c|c|c|c|c|c|c|}
\hline \multirow{2}{*}{$\begin{array}{l}\text { Event } \\
\text { Event }\end{array}$} & \multicolumn{2}{|c|}{ Year at the event } & \multirow[b]{2}{*}{ Source } & \multirow[t]{2}{*}{ Subsequent years } & \multirow[b]{2}{*}{ Unit price } & \multirow[b]{2}{*}{ Source } & \multirow[b]{2}{*}{ Dosage } & \multirow[b]{2}{*}{ Source } & \multirow[b]{2}{*}{$\begin{array}{l}\text { Total } \\
\text { annual } \\
\text { costs }\end{array}$} \\
\hline & Cost (Fatal) & $\begin{array}{l}\text { Cost } \\
\text { (Non-Fatal) }\end{array}$ & & & & & & & \\
\hline \multirow[t]{9}{*}{ IHD } & $\$ 16,861.65$ & $\$ 16,861.65$ & $\begin{array}{l}\text { Strategic overview } \\
\text { cardiovascular } \\
\text { disease in NZ, } 2013\end{array}$ & $\begin{array}{l}\text { Cilazapril with } \\
\text { hydrochlotothiazide }\end{array}$ & $\begin{array}{l}5 \mathrm{mg} \text { per tab; } \\
\$ 10.8 \text { per } 100 \\
\text { tab }\end{array}$ & $\begin{array}{l}\text { PHARMAC } \\
\text { online } \\
\text { schedule }\end{array}$ & $5 \mathrm{mg} / \mathrm{d}$ & $\begin{array}{l}\text { Primary care } \\
\text { handbook; } \\
\text { Medsafe }\end{array}$ & $\$ 39.42$ \\
\hline & & & & Metoprolol & $\begin{array}{l}100 \mathrm{mg} \text { per } \\
\text { tab; } \$ 7.55 \text { per } \\
60 \text { tab }\end{array}$ & $\begin{array}{l}\text { PHARMAC } \\
\text { online } \\
\text { schedule } \\
\end{array}$ & $100 \mathrm{mg} / \mathrm{d}$ & $\begin{array}{l}\text { Primary care } \\
\text { handbook; } \\
\text { Medsafe }\end{array}$ & $\$ 45.93$ \\
\hline & & & & Statin & $\begin{array}{l}\text { 40mg per tab: } \\
\$ 15.93 \text { per } \\
500\end{array}$ & $\begin{array}{l}\text { PHARMAC } \\
\text { online } \\
\text { schedule }\end{array}$ & $40 \mathrm{mg} / \mathrm{d}$ & $\begin{array}{l}\text { Primary care } \\
\text { handbook; } \\
\text { Medsafe }\end{array}$ & $\$ 11.63$ \\
\hline & & & & Marevan & $\begin{array}{l}1 \mathrm{mg} \text { per tab; } \\
\$ 7.6 \text { per } 100 \\
\text { tab }\end{array}$ & $\begin{array}{l}\text { PHARMAC } \\
\text { online } \\
\text { schedule }\end{array}$ & $6 \mathrm{mg} / \mathrm{d}$ & $\begin{array}{l}\text { Primary care } \\
\text { handbook; } \\
\text { Medsafe }\end{array}$ & $\$ 166.44$ \\
\hline & & & & Aspirin & $\begin{array}{l}\text { 100mg per } \\
\text { tab; } \$ 10.8 \text { per } \\
990\end{array}$ & $\begin{array}{l}\text { PHARMAC } \\
\text { online } \\
\text { schedule }\end{array}$ & $100 \mathrm{mg} / \mathrm{d}$ & $\begin{array}{l}\text { Primary care } \\
\text { handbook; } \\
\text { Medsafe }\end{array}$ & $\$ 3.98$ \\
\hline & & & & Clopidogrel & $\begin{array}{l}75 \mathrm{mg} \text { per tab; } \\
5.44 \text { per } 84 \\
\text { tab }\end{array}$ & $\begin{array}{l}\text { PHARMAC } \\
\text { online } \\
\text { schedule }\end{array}$ & $75 \mathrm{mg} / \mathrm{d}$ & $\begin{array}{l}\text { Primary care } \\
\text { handbook; } \\
\text { Medsafe }\end{array}$ & $\$ 23.64$ \\
\hline & & & & Cardiacology & $\begin{array}{l}\$ 285.31 \text { per } \\
\text { time }\end{array}$ & $\mathrm{PU}$ & $\begin{array}{l}4 \quad \text { visits } \\
\text { per year }\end{array}$ & Expert opinion & $\$ 1,158.36$ \\
\hline & & & & Full blood count & $\$ 8.55$ per test & PU & 4 tests & Expert opinion & $\$ 34.71$ \\
\hline & & & & None-hospital cost & $\$ 1,484.11$ & & & & \\
\hline $\begin{array}{l}\text { Total cost at } \\
\text { the event }\end{array}$ & $\$ 16,861.65$ & $\$ 16,861.65$ & & & & & & & \\
\hline $\begin{array}{l}\text { Annual cost } \\
\text { after year } 1\end{array}$ & $\$ 1,484.11$ & $\$ 1,484.11$ & & & & & & & \\
\hline
\end{tabular}


Table 5.5 Costs of IHD the Most Conservative Situation

\begin{tabular}{|c|c|c|c|c|c|c|c|c|c|}
\hline Event & \multicolumn{2}{|c|}{ Year at the event } & \multirow[t]{2}{*}{ Source } & \multirow[t]{2}{*}{ Subsequent years } & \multirow[b]{2}{*}{ Unit price } & \multirow[b]{2}{*}{ Source } & \multirow[b]{2}{*}{ Dosage } & \multirow[b]{2}{*}{ Source } & \multirow[b]{2}{*}{$\begin{array}{l}\text { Total } \\
\text { annual } \\
\text { costs }\end{array}$} \\
\hline Event & $\begin{array}{l}\text { Cost } \\
\text { (Fatal) }\end{array}$ & $\begin{array}{l}\text { Cost } \\
\text { (Non-Fatal } \\
\text { ) }\end{array}$ & & & & & & & \\
\hline \multirow[t]{8}{*}{ IHD } & $\$ 7,494.07$ & $\$ 7,494.07$ & $\begin{array}{l}\text { Strategic overview } \\
\text { cardiovascular } \\
\text { disease in NZ, } \\
2013\end{array}$ & $\begin{array}{l}\text { Cilazapril with } \\
\text { hydrochlotothiazide }\end{array}$ & $\begin{array}{l}\text { 5mg per tab; } \\
\$ 10.8 \text { per } \\
100 \text { tab }\end{array}$ & $\begin{array}{l}\text { PHARMAC } \\
\text { online } \\
\text { schedule }\end{array}$ & $5 \mathrm{mg} / \mathrm{d}$ & $\begin{array}{l}\text { Primary care } \\
\text { handbook; } \\
\text { Medsafe }\end{array}$ & $\$ 39.42$ \\
\hline & & & & Metoprolol & $\begin{array}{lr}50 m g & \text { per } \\
\text { tab; } \$ 5.66 \\
\text { per } 100 \text { tab }\end{array}$ & $\begin{array}{l}\text { PHARMAC } \\
\text { online } \\
\text { schedule }\end{array}$ & $50 \mathrm{mg} / \mathrm{d}$ & $\begin{array}{l}\text { Primary care } \\
\text { handbook; } \\
\text { Medsafe }\end{array}$ & $\$ 20.66$ \\
\hline & & & & Statin & $\begin{array}{l}\text { 20mg per } \\
\text { tab: } \$ 9.99 \\
\text { per } 500 \text { tab }\end{array}$ & $\begin{array}{l}\text { PHARMAC } \\
\text { online } \\
\text { schedule }\end{array}$ & $20 \mathrm{mg} / \mathrm{d}$ & $\begin{array}{l}\text { Primary care } \\
\text { handbook; } \\
\text { Medsafe }\end{array}$ & $\$ 7.29$ \\
\hline & & & & Marevan & $\begin{array}{l}\text { 1mg per tab; } \\
\$ 7.6 \text { per } 100 \\
\text { tab }\end{array}$ & $\begin{array}{l}\text { PHARMAC } \\
\text { online } \\
\text { schedule }\end{array}$ & $2 \mathrm{mg} / \mathrm{d}$ & $\begin{array}{l}\text { Primary care } \\
\text { handbook; } \\
\text { Medsafe }\end{array}$ & $\$ 55.48$ \\
\hline & & & & Aspirin & $\begin{array}{l}\text { 100mg per } \\
\text { tab; } \$ 10.8 \\
\text { per } 990\end{array}$ & $\begin{array}{l}\text { PHARMAC } \\
\text { online } \\
\text { schedule } \\
\end{array}$ & $100 \mathrm{mg} / \mathrm{d}$ & $\begin{array}{l}\text { Primary care } \\
\text { handbook; } \\
\text { Medsafe }\end{array}$ & $\$ 3.98$ \\
\hline & & & & Cardiacology & $\begin{array}{l}\$ 285.31 \text { per } \\
\text { time }\end{array}$ & PU & $\begin{array}{l}1 \text { visits } \\
\text { per year }\end{array}$ & Expert opinion & $\$ 289.59$ \\
\hline & & & & Full blood count & $\begin{array}{l}\$ 8.55 \text { per } \\
\text { test }\end{array}$ & $\mathrm{PU}$ & 1 tests & Expert opinion & $\$ 8.68$ \\
\hline & & & & Total none-DRG cost & $\$ 425.1$ & & & & \\
\hline $\begin{array}{l}\text { Total cost at } \\
\text { the event }\end{array}$ & $\$ 7,497.07$ & $\$ 7,497.07$ & & & & & & & \\
\hline $\begin{array}{l}\text { Annual cost } \\
\text { after year } 1\end{array}$ & $\$ 425.1$ & & & & & & & & \\
\hline
\end{tabular}


Table 5.6 Costs of IHD: Base Case

\begin{tabular}{|c|c|c|c|c|c|c|c|c|c|}
\hline $\begin{array}{l}\text { Event } \\
\text { Event }\end{array}$ & \multicolumn{2}{|c|}{ Year at the event } & Source & Subsequent years & Unit price & Source & Dosage & Source & $\begin{array}{l}\text { Total } \\
\text { annual } \\
\text { costs }\end{array}$ \\
\hline \multirow[t]{7}{*}{ IHD } & $\$ 11,241.10$ & $\$ 11,241.10$ & $\begin{array}{l}\text { Strategic overview } \\
\text { cardiovascular } \\
\text { disease in NZ, } 2013\end{array}$ & $\begin{array}{l}\text { Cilazapril with } \\
\text { hydrochlotothiazide }\end{array}$ & $\begin{array}{l}5 \mathrm{mg} \text { per } \\
\text { tab; } \\
\$ 10.8 \text { per } \\
100 \text { tab }\end{array}$ & $\begin{array}{l}\text { PHARMAC } \\
\text { online } \\
\text { schedule }\end{array}$ & $5 \mathrm{mg} / \mathrm{d}$ & $\begin{array}{l}\text { Primary care } \\
\text { handbook; } \\
\text { Medsafe }\end{array}$ & $\$ 39.42$ \\
\hline & & & & Metoprolol & $\begin{array}{l}\text { 50mg per } \\
\text { tab; } \$ 5.66 \\
\text { per } 100 \text { tab }\end{array}$ & $\begin{array}{l}\text { PHARMAC } \\
\text { online } \\
\text { schedule }\end{array}$ & $75 \mathrm{mg} / \mathrm{d}$ & $\begin{array}{l}\text { Primary care } \\
\text { handbook; } \\
\text { Medsafe }\end{array}$ & $\$ 30.99$ \\
\hline & & & & Statin & $\begin{array}{l}\text { 20mg per } \\
\text { tab: } \$ 9.99 \\
\text { per } 500 \text { tab }\end{array}$ & $\begin{array}{l}\text { PHARMAC } \\
\text { online } \\
\text { schedule }\end{array}$ & $30 \mathrm{mg} / \mathrm{d}$ & $\begin{array}{l}\text { Primary care } \\
\text { handbook; } \\
\text { Medsafe }\end{array}$ & $\$ 10.94$ \\
\hline & & & & Marevan & $\begin{array}{lr}1 \mathrm{mg} & \text { per } \\
\text { tab; } & \$ 7.6 \\
\text { per } 100 \text { tab }\end{array}$ & $\begin{array}{l}\text { PHARMAC } \\
\text { online } \\
\text { schedule }\end{array}$ & $4 \mathrm{mg} / \mathrm{d}$ & $\begin{array}{l}\text { Primary care } \\
\text { handbook; } \\
\text { Medsafe }\end{array}$ & $\$ 110.96$ \\
\hline & & & & Aspirin & $\begin{array}{l}\text { 100mg per } \\
\text { tab; } \$ 10.8 \\
\text { per } 990\end{array}$ & $\begin{array}{l}\text { PHARMAC } \\
\text { online } \\
\text { schedule }\end{array}$ & $100 \mathrm{mg} / \mathrm{d}$ & $\begin{array}{l}\text { Primary care } \\
\text { handbook; } \\
\text { Medsafe }\end{array}$ & $\$ 3.98$ \\
\hline & & & & Full blood count & $\begin{array}{l}\$ 8.55 \text { per } \\
\text { test }\end{array}$ & $P U$ & 3 tests & $\begin{array}{l}\text { Expert } \\
\text { opinion }\end{array}$ & $\$ 26.04$ \\
\hline & & & & Total none-DRG cost & $\$ 425$ & & & & \\
\hline $\begin{array}{l}\text { Total cost at } \\
\text { the event }\end{array}$ & $\$ 11,241.10$ & $\$ 11,241.10$ & & & & & & & \\
\hline $\begin{array}{l}\text { Annual cost } \\
\text { after year } 1\end{array}$ & $\$ 1,091.1$ & & & & & & & & \\
\hline
\end{tabular}


Appendix IV: Information for the UKPDS Model

Table 5.8 Costs of Dialysis in New Zealand

\begin{tabular}{|c|c|c|c|c|}
\hline Type of dialysis & Type of management & Proportion & Initial costs & Following costs \\
\hline \multicolumn{5}{|l|}{ Hemodialysis } \\
\hline Center & & $53 \%$ & & \\
\hline & Self-manage & $26.5 \%$ & $\$ 3,807.67$ & $\$ 261.61$ per time \\
\hline & Non-self-manage & $26.5 \%$ & & $\$ 422.21$ per time \\
\hline Home & & $16 \%$ & $\$ 1,6721.88$ & $\$ 2,771.66$ per month \\
\hline \multicolumn{5}{|l|}{ Peritoneal } \\
\hline Center & & $12 \%$ & & 51 per time \\
\hline Home & & $19 \%$ & $\$ 3,040.13$ & $\$ 257.81$ per month \\
\hline
\end{tabular}

Table 5.10: UKPDS Results: Diabetes Costs without Complications, Costs of Complications \& the Possible Range of Total Costs

\begin{tabular}{|c|c|c|c|c|c|c|c|c|}
\hline & & Base Case & & Lower bound & & Higher bound & & $\begin{array}{l}\text { Min and Max Total Costs: } \\
(95 \% \mathrm{Cl})\end{array}$ \\
\hline & & $\begin{array}{l}\text { Cost at the } \\
\text { event }\end{array}$ & $\begin{array}{l}\text { Subsequent } \\
\text { years }\end{array}$ & $\begin{array}{l}\text { Cost at the } \\
\text { event }\end{array}$ & $\begin{array}{l}\text { Subsequent } \\
\text { years }\end{array}$ & $\begin{array}{l}\text { Cost at the } \\
\text { event }\end{array}$ & $\begin{array}{l}\text { Subsequent } \\
\text { years }\end{array}$ & \\
\hline $\begin{array}{l}\text { Cost in } \\
\text { absence of } \\
\text { complications }\end{array}$ & & $\$ 941.91$ & & $\$ 168.7$ & & $\$ 2,423.11$ & & $\begin{array}{l}\$ 17,510.92(\$ 12,136.13- \\
\$ 22,885.71) ; \\
\$ 54,900.98 \quad(\$ 49,219.79- \\
\$ 60,582.18)\end{array}$ \\
\hline \multicolumn{9}{|l|}{$\begin{array}{l}\text { Costs of } \\
\text { complications }\end{array}$} \\
\hline \multirow[t]{2}{*}{ IHD } & Non-Fatal & $\$ 11,241.10$ & $\$ 1,091.1$ & $\$ 5,620.55$ & $\$ 425.1$ & $\$ 16,861.66$ & $\$ 1,484.11$ & $\$ 29,496.63 \quad(\$ 24,145.77 \quad-$ \\
\hline & Fatal & $\$ 11,241.10$ & $\$ 0$ & $\$ 5,620.55$ & $\$ 0$ & $\$ 16,861.66$ & $\$ 0$ & $\begin{array}{l}\$ 34,847.48) ; \\
\$ 30,967.34 \quad(\$ 25,491.92- \\
\$ 36,442.75)\end{array}$ \\
\hline $\mathrm{MI}$ & Non-Fatal & $\$ 12,394.87$ & $\$ 1,118.2$ & $\$ 11,725.41$ & $\$ 448.74$ & $\$ 15,146.92$ & $\$ 3,870.25$ & $\$ 29,739.37 \quad(\$ 24,344.60 \quad-$ \\
\hline
\end{tabular}




\begin{tabular}{|c|c|c|c|c|c|c|c|c|}
\hline & Fatal & $\$ 14,737.58$ & $\$ 0$ & $\$ 7,368.79$ & $\$ 0$ & $\$ 22,106.37$ & $\$ 0$ & $\begin{array}{l}\$ 35,134.14) ; \\
\$ 32,449.45 \quad(\$ 26,910.98 \\
\$ 37,987.93)\end{array}$ \\
\hline \multirow[t]{2}{*}{ Stroke } & Non-Fatal & $\$ 30,890.38$ & $\$ 13,983.08$ & $\$ 5,162.89$ & $\$ 158.05$ & $\$ 52,472.21$ & $\$ 34,946.13$ & \multirow{2}{*}{$\begin{array}{l}\$ 27,588.68 \quad(\$ 22,447.72 \\
\$ 32,729.64) ; \\
\$ 34,217.18 \quad(\$ 27,757.35 \\
\$ 40,677.01)\end{array}$} \\
\hline & Fatal & $\$ 3,470.02$ & $\$ 0$ & $\$ 1,735.01$ & $\$ 0$ & $\$ 5,205.03$ & $\$ 0$ & \\
\hline \multirow[t]{2}{*}{ Renal Failure } & Non-Fatal & $\$ 30,900.89$ & $\$ 26,574.82$ & $\$ 20,602.5$ & $\$ 16,286.43$ & $\$ 48,713.87$ & $\$ 37,237.04$ & \multirow{2}{*}{$\begin{array}{l}\$ 28,878.15 \quad(\$ 24,528.07 \\
\$ 33,228.23) ; \\
\$ 31,956.74 \quad(\$ 25,14.19 \\
\$ 38799.03)\end{array}$} \\
\hline & Fatal & $\$ 12,668.48$ & $\$ 0$ & $\$ 6,344.24$ & $\$ 0$ & $\$ 19,032.72$ & $\$ 0$ & \\
\hline Blindness & & $\$ 18,637.86$ & $\$ 18,229.14$ & $\$ 9,523.29$ & $\$ 9,114.57$ & $\$ 29,304.12$ & $\$ 28,895.4$ & $\begin{array}{l}\$ 28,289.62 \quad(\$ 23,462.66 \\
\$ 33,116.58) ; \\
\$ 32,728.28 \quad(\$ 26,363.68 \\
\$ 39,092.89)\end{array}$ \\
\hline \multirow[t]{2}{*}{ Amputation } & None-Fatal & $\$ 19,828.75$ & $\$ 0$ & $\$ 9,914.38$ & $\$ 0$ & $\$ 29,743.13$ & $\$ 0$ & \multirow{2}{*}{$\begin{array}{l}\$ 30,164.33 \quad(\$ 24,767.29 \\
\$ 35,561.36) ; \\
\$ 30505.35 \quad(\$ 25,063.50 \\
\$ 35,947.2)\end{array}$} \\
\hline & Fatal & $\$ 27,443.95$ & $\$ 0$ & $\$ 13,721.98$ & $\$ 0$ & $\$ 41,165.93$ & $\$ 0$ & \\
\hline \multirow[t]{2}{*}{ Heart Failure } & None-Fatal & $\$ 8,445.19$ & $\$ 1,338.13$ & $\$ 5,853.8$ & $\$ 399.48$ & $\$ 12,559.4$ & $\$ 3,799.61$ & \multirow{2}{*}{$\begin{array}{l}\$ 30,112.8 \quad(\$ 24,727.18 \\
\$ 35,518.42) ; \\
\$ 30,842.18 \quad(\$ 25,358.08 \\
36,326.29)\end{array}$} \\
\hline & Fatal & $\$ 3,344.72$ & $\$ 0$ & $\$ 1,672.36$ & $\$ 0$ & $\$ 5,017.08$ & $\$ 0$ & \\
\hline
\end{tabular}

Table 5.21 Costs of Amputation

\begin{tabular}{|c|c|c|c|c|c|}
\hline Event & Year at the event & & & & \\
\hline & DRG Units (Fatal) & DRG Units (Non-Fatal) & Source & DRG Unit price & Source \\
\hline Amputation & 5.3350 & 3.8546 & 2018 DRG & $\$ 5,068.12$ & MoH Website \\
\hline DRG total cost & $\$ 27,443.95$ & $\$ 19,828.75$ & & & \\
\hline Total cost at the event & $\$ 27,443.95$ & $\$ 19,828.75$ & & & \\
\hline
\end{tabular}


Table 5.11 New Zealand Diabetes Patient Population \& Incidence by Year

\begin{tabular}{|c|c|c|c|c|c|c|c|c|c|}
\hline Year & 2010 & 2011 & 2012 & 2013 & 2014 & 2015 & 2016 & 2017 & 2018 \\
\hline Patients & 187,860 & 200,235 & 211,591 & 220,866 & 228,790 & 236,073 & 241,463 & 245,680 & 253,480 \\
\hline New Patients & & 12375 & 11356 & 9275 & 7924 & 7283 & 5390 & 4217 & 7800 \\
\hline Type 2 & & 11138 & 10220 & 8348 & 7132 & 6555 & 4851 & 3795 & 7020 \\
\hline NZ Population & $4,351,000$ & $4,384,000$ & $4,408,000$ & $4,442,000$ & $4,510,000$ & $4,596,000$ & $4,693,000$ & $4,794,000$ & $4,841,000$ \\
\hline Incidence & & 0.002541 & 0.002318 & 0.001879 & 0.001581 & 0.001427 & 0.001034 & 0.00079 & 0.001450 \\
\hline
\end{tabular}

Table 5.12: Formulas in the UKPDS model

\begin{tabular}{|c|c|}
\hline Variable & Formula \\
\hline IHD & $1.15 * \mathrm{t}^{0.015 *} \mathrm{e}^{(-5.310+0.031 * \mathrm{AGE}-0.471 * \mathrm{FEMALE}+0.125 * \mathrm{HBA} 1 \mathrm{C}+0.098 * \mathrm{ABP}+1.498 * \operatorname{Ln}(\mathrm{TOTAL}: \mathrm{HDL})}$ \\
\hline MI & $1.15 * \mathrm{t}^{0.015 *} \mathrm{e}^{(-5.310+0.031 * \mathrm{AGE}-0.471 * \mathrm{FEMALE}+0.125 * \mathrm{HBA} 1 \mathrm{C}+0.098 * \mathrm{ABP}+1.498 * \operatorname{Ln}(\mathrm{TOTAL}: \mathrm{HDL})}$ \\
\hline Heart Failure & $1.15 * \mathrm{t}^{0.015 *} \mathrm{e}^{(-5.310+0.031 * \mathrm{AGE}-0.471 * \mathrm{FEMALE}+0.125 * \mathrm{HBA} 1 \mathrm{C}+0.098 * \mathrm{ABP}+1.498 * \operatorname{Ln}(\mathrm{TOTAL}: \mathrm{HDL})}$ \\
\hline Stroke & $1.15 * \mathrm{t}^{0.015 *} \mathrm{e}^{(-5.310+0.031 * \mathrm{AGE}-0.471 * \mathrm{FEMALE}+0.125 * \mathrm{HBA} 1 \mathrm{C}+0.098 * \mathrm{ABP}+1.498 * \operatorname{Ln}(\mathrm{TOTAL}: \mathrm{HDL})}$ \\
\hline Amputation & $1.15 * \mathrm{t}^{0.015 *} \mathrm{e}^{(-5.310+0.031 * \mathrm{AGE}-0.471 * \mathrm{FEMALE}+0.125 * \mathrm{HBA} 1 \mathrm{C}+0.098 * \mathrm{ABP}+1.498 * \operatorname{Ln}(\mathrm{TOTAL}: \mathrm{HDL})}$ \\
\hline Blindness & $1.15 * \mathrm{t}^{0.015 *} \mathrm{e}^{(-5.310+0.031 * \mathrm{AGE}-0.471 * \mathrm{FEMALE}+0.125 * \mathrm{HBA} 1 \mathrm{C}+0.098 * \mathrm{ABP}+1.498 * \operatorname{Ln}(\mathrm{TOTAL}: \mathrm{HDL})}$ \\
\hline Renal Failure & $1.15 * \mathrm{t}^{0.015 *} \mathrm{e}^{(-5.310+0.031 * \mathrm{AGE}-0.471 * \mathrm{FEMALE}+0.125 * \mathrm{HBA} 1 \mathrm{C}+0.098 * \mathrm{ABP}+1.498 * \operatorname{Ln}(\mathrm{TOTAL}: \mathrm{HDL})}$ \\
\hline $\begin{array}{l}\text { Diabetes related death } \\
\text { in the first year }\end{array}$ & $1 /\left(1+\mathrm{e}^{(-3.251+2.772 * \text { LN(AGE_EVENT)+0.114*HBA1C+2.640*MI_EVENT+1.048*STROKE_EVENT) })}\right.$ \\
\hline $\begin{array}{l}\text { Diabetes related death } \\
\text { in the subsequent years }\end{array}$ & $\mathrm{e}^{(-5.124+0.003 * t+4.731 * \text { LN(AGE_EVENT)+0.109*TOTAL:HDL+3.939MI_EVENT+1.119*MI_POST+2.807*STROKE_EVENT+1.585*RENAL+1.032*AMP) })}$ \\
\hline $\begin{array}{l}\text { Non-diabetes related } \\
\text { death }\end{array}$ & $\mathrm{e}^{(-6.373+0.154 * \mathrm{t}+0.081 * A G E * \text { FEMALE }+0.104 * A G E *(1-\mathrm{FEMALE})+0.307 * \mathrm{SMOKE})}$ \\
\hline HbA1c & $-0.024+0.144 * \operatorname{Ln}($ Year)-0.333*Year_2+0.759*LHBA1C+0.085*HBA1C_BASE \\
\hline Blood Pressure & $0.03+0.039 * \operatorname{Ln}(Y E A R)+0.717 *$ LSBP+0.127*SBP_BASE \\
\hline HDL & -0.021+0.526*LTOTAL:HDL+0.252*LTOT:HDL_BASE \\
\hline Smoke & 1/(1+e(-4.02-0.203*YEAR-0.027*AGE-0.489*FEMALE+1.878*LSSMOKE+4.879*SMOKE_BASE) \\
\hline
\end{tabular}


Table 5.13 Variable Definition in the UKPDS Model

\begin{tabular}{|c|c|}
\hline Variable & Definition \\
\hline AGE & Age at diagnosed diabetes \\
\hline AGE_EVENT & Age at the first diabetic complication occurs \\
\hline Year & Time from diagnosed diabetes to current year \\
\hline Year_2 & $=1$, if it is the second year after diagnosed diabetes; $=0$, otherwise \\
\hline FEMALE & $=1$, if female; $=0$, otherwise \\
\hline $\mathrm{AC}$ & $=1$, if African; =0, if European or Asian \\
\hline SMOKE & $=1$, if smoke; $=0$, otherwise \\
\hline SMOKE_BASE & $=1$ if smoke when diagnosed diabetes; =0, otherwise \\
\hline BMI & Body-mas index when diagnosed diabetes \\
\hline HBA1C & The average of HBA1c in the last two years \\
\hline LHBA1C & The average of HBA1c last year \\
\hline HBA1C_BASE & HBA1c when diagnosed diabetes \\
\hline SBP & The average systolic blood pressure in the last two years \\
\hline LSBP & The average systolic blood pressure last year \\
\hline SBP_BASE & Systolic blood pressure when diagnosed diabetes \\
\hline TOTAL:HDL & The average proportion of high dense cholesterol in the last two years \\
\hline LTOTL:HDL & The average proportion of high dense cholesterol last year \\
\hline LTOTL:HDL_BASE & The average proportion of high dense cholesterol when diagnosed diabetes \\
\hline ATRFIB & $=1$, had atrial fibrillation when diagnosed diabetes; =0, otherwise \\
\hline PVD & $=1$, had peripheral vascular when diagnosed diabetes; $=0$, otherwise \\
\hline IHD & $=1$, had IHD history; $=0$, otherwise \\
\hline MI_EVENT & $=1$, had MI history; $=0$, otherwise \\
\hline MI_POST & $=1$, one - year after $\mathrm{Ml} ;=0$, otherwise \\
\hline STROKE_EVENT & $=1$, if stroke occurs at the year; $=0$, otherwise \\
\hline $\mathrm{CHF}$ & $=1$, if had coronary heart failure; $=0$, otherwise \\
\hline AMP & $=1$, if had amputation history; $=0$, otherwise \\
\hline BLIND & $=1$, if had blinded; $=0$, otherwise \\
\hline RENAL & $=1$, if had renal failure history; $=0$, otherwise \\
\hline
\end{tabular}


Table 5.14: Costs of Heart Failure: the Most Serious Situation

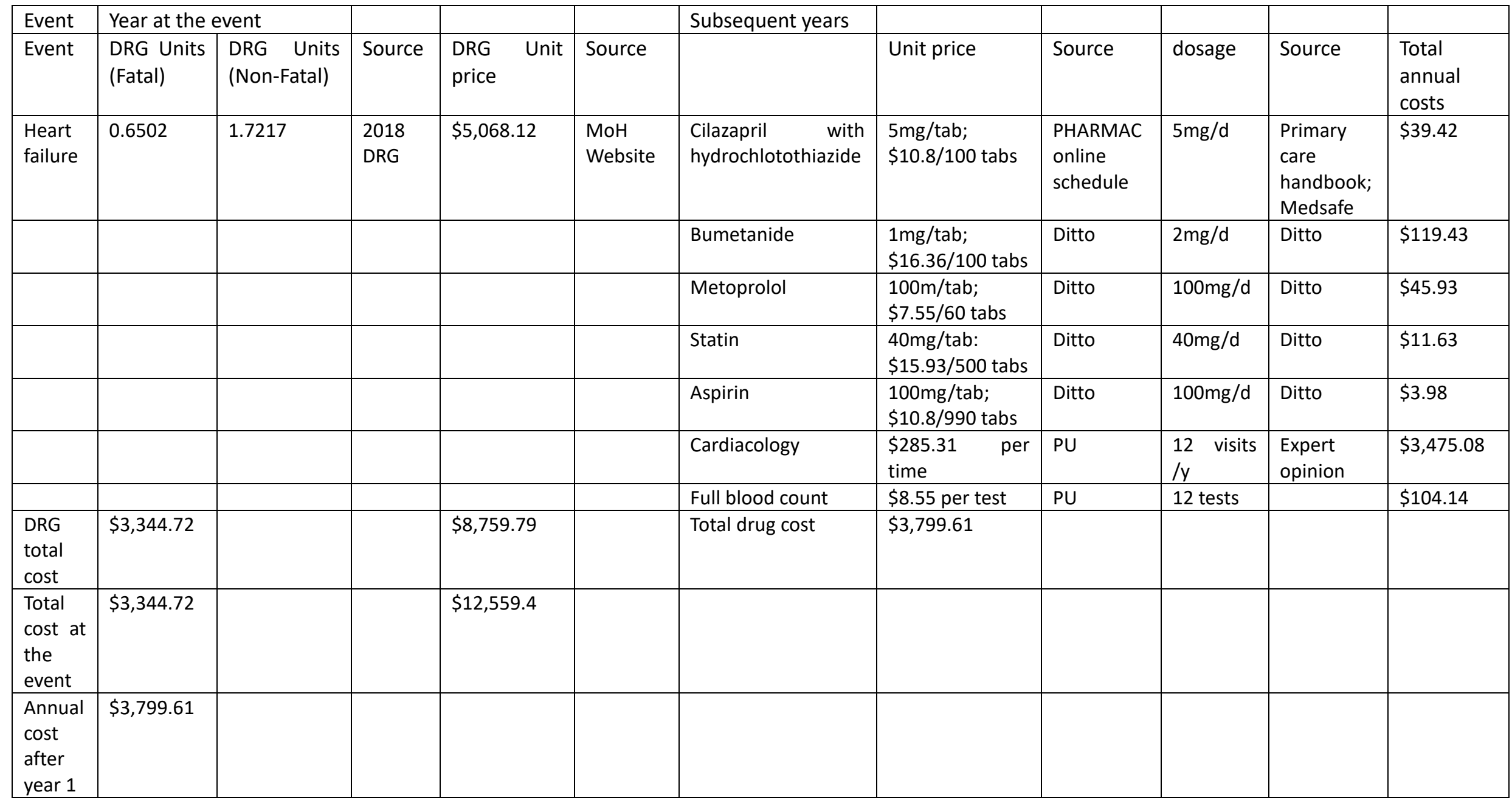


Table 5.15 Costs of Heart Failure: The Most Conservative Situation

\begin{tabular}{|c|c|c|c|c|c|c|c|c|c|c|c|}
\hline \multirow{2}{*}{$\frac{\text { Event }}{\text { Event }}$} & \multicolumn{2}{|c|}{ Year at the event } & \multirow[b]{2}{*}{ Source } & \multirow[b]{2}{*}{$\begin{array}{l}\text { DRG Unit } \\
\text { price }\end{array}$} & \multirow[b]{2}{*}{ Source } & \multirow[t]{2}{*}{ Subsequent years } & \multirow[b]{2}{*}{ Unit price } & \multirow[b]{2}{*}{ Source } & \multirow[b]{2}{*}{ dosage } & \multirow[b]{2}{*}{ Source } & \multirow[b]{2}{*}{$\begin{array}{l}\text { Total } \\
\text { annual } \\
\text { costs }\end{array}$} \\
\hline & $\begin{array}{l}\text { DRG Units } \\
\text { (Fatal) }\end{array}$ & $\begin{array}{l}\text { DRG Units } \\
\text { (Non- } \\
\text { Fatal) }\end{array}$ & & & & & & & & & \\
\hline \multirow[t]{7}{*}{ Heart failure } & 0.6502 & 1.0603 & $\begin{array}{l}2018 \\
\text { DRG }\end{array}$ & $\$ 5,068.12$ & $\begin{array}{l}\text { MoH } \\
\text { Website }\end{array}$ & $\begin{array}{l}\text { Cilazapril with } \\
\text { hydrochlotothiazide }\end{array}$ & $\begin{array}{l}5 \mathrm{mg} \text { per tab; } \\
\$ 10.8 \text { per } 100 \\
\text { tab }\end{array}$ & $\begin{array}{l}\text { PHARMAC } \\
\text { online } \\
\text { schedule }\end{array}$ & $5 \mathrm{mg} / \mathrm{d}$ & $\begin{array}{l}\text { Primary care } \\
\text { handbook; } \\
\text { Medsafe }\end{array}$ & $\$ 39.42$ \\
\hline & & & & & & Bumetanide & $\begin{array}{l}1 \mathrm{mg} / \mathrm{tab} ; \\
\$ 16.36 / 100 \text { tabs }\end{array}$ & Ditto & $0.5 \mathrm{mg} / \mathrm{d}$ & Ditto & $\$ 29.86$ \\
\hline & & & & & & Metoprolol & $\begin{array}{l}50 \mathrm{mg} / \mathrm{tab} ; \\
\$ 5.66 / 100 \text { tabs }\end{array}$ & Ditto & $50 \mathrm{mg} / \mathrm{d}$ & Ditto & $\$ 20.66$ \\
\hline & & & & & & Statin & $\begin{array}{l}\text { 20mg/tab: } \\
\$ 9.99 / 500 \text { tabs }\end{array}$ & Ditto & $20 \mathrm{mg} / \mathrm{d}$ & Ditto & $\$ 7.29$ \\
\hline & & & & & & Aspirin & $\begin{array}{l}100 \mathrm{mg} / \mathrm{tab} ; \\
\$ 10.8 / 990 \text { tabs }\end{array}$ & Ditto & $100 \mathrm{mg} / \mathrm{d}$ & Ditto & $\$ 3.98$ \\
\hline & & & & & & Cardiacology & $\begin{array}{l}\$ 285.31 \quad \text { per } \\
\text { time }\end{array}$ & PU & $\begin{array}{l}1 \text { visit } \\
\text { per year }\end{array}$ & $\begin{array}{l}\text { Expert } \\
\text { opinion }\end{array}$ & $\$ 289.59$ \\
\hline & & & & & & Full blood count & $\$ 8.55$ per test & PU & 1 test & Ditto & $\$ 8.68$ \\
\hline DRG total cost & $\$ 3,344.72$ & $\$ 5,454.32$ & & & & Total drug cost & $\$ 399.48$ & & & & \\
\hline $\begin{array}{l}\text { Total cost at } \\
\text { the event }\end{array}$ & $\$ 3,344.72$ & $\$ 5,853.8$ & & & & & & & & & \\
\hline $\begin{array}{l}\text { Annual cost } \\
\text { after year } 1\end{array}$ & $\$ 399.48$ & & & & & & & & & & \\
\hline
\end{tabular}


Table 5.16: Costs of Heart Failure: Base Case

\begin{tabular}{|c|c|c|c|c|c|c|c|c|c|c|c|}
\hline \multirow{2}{*}{$\begin{array}{l}\text { Event } \\
\text { Event }\end{array}$} & \multicolumn{2}{|c|}{ Year at the event } & \multirow[b]{2}{*}{ Source } & \multirow[b]{2}{*}{$\begin{array}{l}\text { DRG Unit } \\
\text { price }\end{array}$} & \multirow[b]{2}{*}{ Source } & \multirow[t]{2}{*}{ Subsequent years } & \multirow[b]{2}{*}{ Unit price } & \multirow[b]{2}{*}{ Source } & \multirow[b]{2}{*}{ Dosage } & \multirow[b]{2}{*}{ Source } & \multirow[b]{2}{*}{$\begin{array}{l}\text { Total } \\
\text { annual } \\
\text { costs }\end{array}$} \\
\hline & $\begin{array}{l}\text { DRG Units } \\
\text { (Fatal) }\end{array}$ & $\begin{array}{l}\text { DRG Units } \\
\text { (Non-Fatal) }\end{array}$ & & & & & & & & & \\
\hline \multirow[t]{7}{*}{$\begin{array}{l}\text { Heart } \\
\text { failure }\end{array}$} & 0.6502 & 1.391 & $\begin{array}{l}2018 \\
\text { DRG }\end{array}$ & $\$ 5,068.12$ & $\begin{array}{l}\text { MoH } \\
\text { Website }\end{array}$ & $\begin{array}{l}\text { Cilazapril with } \\
\text { hydrochlotothiazide }\end{array}$ & $\begin{array}{l}5 \mathrm{mg} / \mathrm{tab} ; \\
\$ 10.8 / 100 \text { tabs }\end{array}$ & $\begin{array}{l}\text { PHARMAC } \\
\text { online } \\
\text { schedule }\end{array}$ & $5 \mathrm{mg} / \mathrm{d}$ & $\begin{array}{l}\text { Primary } \\
\text { care } \\
\text { handbook; } \\
\text { Medsafe }\end{array}$ & $\$ 39.42$ \\
\hline & & & & & & Bumetanide & $\begin{array}{l}\text { 1mg/tab; } \\
\$ 16.36 / 100 \text { tabs }\end{array}$ & Ditto & $1 \mathrm{mg} / \mathrm{d}$ & Ditto & $\$ 59.72$ \\
\hline & & & & & & Metoprolol & $\begin{array}{l}\text { 50mg/tab; } \\
\$ 5.66 / 100 \text { tabs }\end{array}$ & Ditto & $75 \mathrm{mg} / \mathrm{d}$ & Ditto & $\$ 30.99$ \\
\hline & & & & & & Statin & $\begin{array}{l}\text { 20mg/tab: } \\
\$ 9.99 / 500 \text { tabs }\end{array}$ & Ditto & $30 \mathrm{mg} / \mathrm{d}$ & Ditto & $\$ 10.94$ \\
\hline & & & & & & Aspirin & $\begin{array}{l}\text { 100mg/tab; } \\
\$ 10.8 / 990 \text { tabs }\end{array}$ & Ditto & $100 \mathrm{mg} / \mathrm{d}$ & Ditto & $\$ 3.98$ \\
\hline & & & & & & Cardiacology & $\$ 285.31 /$ time & PU & 4/year & $\begin{array}{l}\text { Expert } \\
\text { opinion }\end{array}$ & $\$ 1,158.36$ \\
\hline & & & & & & Full blood count & $\$ 8.55 /$ test & PU & 4 tests/y & Ditto & $\$ 34.72$ \\
\hline $\begin{array}{l}\text { DRG total } \\
\text { cost }\end{array}$ & $\$ 3,344.72$ & $\$ 7,107.06$ & & & & Total drug cost & $\$ 1,338.13$ & & & & \\
\hline $\begin{array}{l}\text { Cost at the } \\
\text { event }\end{array}$ & $\$ 3,344.72$ & $\$ 8,445.19$ & & & & & & & & & \\
\hline $\begin{array}{l}\text { Cost after } \\
\text { year } 1\end{array}$ & $\$ 1,338.13$ & & & & & & & & & & \\
\hline
\end{tabular}


Table 5.17 Costs of MI: the Most Serious Situation

\begin{tabular}{|c|c|c|c|c|c|c|c|c|c|c|c|}
\hline \multirow{2}{*}{$\begin{array}{l}\text { Event } \\
\text { Event }\end{array}$} & \multicolumn{2}{|c|}{ Year at the event } & \multirow[b]{2}{*}{ Source } & \multirow[b]{2}{*}{$\begin{array}{l}\text { DRG Unit } \\
\text { price }\end{array}$} & \multirow[b]{2}{*}{ Source } & \multirow[t]{2}{*}{ Subsequent years } & \multirow[b]{2}{*}{ Unit price } & \multirow[b]{2}{*}{ Source } & \multirow[b]{2}{*}{ dosage } & \multirow[b]{2}{*}{ Source } & \multirow[b]{2}{*}{$\begin{array}{l}\text { Total } \\
\text { annual } \\
\text { costs }\end{array}$} \\
\hline & $\begin{array}{l}\text { DRG Units } \\
\text { (Fatal) }\end{array}$ & $\begin{array}{l}\text { DRG Units } \\
\text { (Non-Fatal) }\end{array}$ & & & & & & & & & \\
\hline \multirow[t]{6}{*}{ MI } & 2.8649 & 2.1921 & $\begin{array}{l}2018 \\
\text { DRG }\end{array}$ & $\$ 5,068.12$ & $\begin{array}{l}\text { MoH } \\
\text { Website }\end{array}$ & $\begin{array}{l}\text { Cilazapril+ } \\
\text { hydrochlotothiazide }\end{array}$ & $\begin{array}{l}\text { 5mg per tab; } \\
\$ 10.8 \text { per } 100 \\
\text { tab }\end{array}$ & $\begin{array}{l}\text { PHARMAC } \\
\text { online } \\
\text { schedule }\end{array}$ & $5 \mathrm{mg} / \mathrm{d}$ & $\begin{array}{l}\text { Primary } \\
\text { care } \\
\text { handbook; } \\
\text { Medsafe }\end{array}$ & $\$ 39.42$ \\
\hline & & & & & & Metoprolol & $\begin{array}{l}\text { 100mg/tab; } \\
\$ 7.55 / 60 \text { tabs }\end{array}$ & Ditto & $100 \mathrm{mg} / \mathrm{d}$ & Ditto & $\$ 45.93$ \\
\hline & & & & & & Statin & $\begin{array}{l}\text { 40mg/tab: } \\
\$ 15.93 / 500 \text { tabs }\end{array}$ & Ditto & $40 \mathrm{mg} / \mathrm{d}$ & Ditto & $\$ 11.63$ \\
\hline & & & & & & Marevan & $\begin{array}{l}1 \mathrm{mg} / \mathrm{tab} ; \\
\$ 7.6 / 100 \text { tabs }\end{array}$ & Ditto & $6 \mathrm{mg} / \mathrm{d}$ & Ditto & $\$ 166.44$ \\
\hline & & & & & & Aspirin & $\begin{array}{l}\text { 100mg/tab; } \\
\$ 10.8 / 990 \text { tabs }\end{array}$ & Ditto & $100 \mathrm{mg} / \mathrm{d}$ & Ditto & $\$ 3.98$ \\
\hline & & & & & & Clopidogrel & $\begin{array}{l}75 \mathrm{mg} \text { per tab; } \\
5.44 \text { per } 84 \text { tab }\end{array}$ & Ditto & $75 \mathrm{mg} / \mathrm{d}$ & Ditto & $\$ 23.64$ \\
\hline $\begin{array}{l}\text { DRG total } \\
\text { cost }\end{array}$ & $\$ 14,737.58$ & $\$ 11,276.67$ & & & & Cardiacology & $\begin{array}{l}\$ 285.31 \quad \text { per } \\
\text { time }\end{array}$ & PU & 12 /year & $\begin{array}{l}\text { Expert } \\
\text { opinion }\end{array}$ & $\$ 3,475.08$ \\
\hline $\begin{array}{l}\text { Cost at the } \\
\text { event }\end{array}$ & $\$ 14,737.58$ & $\$ 15,146.92$ & & & & Full blood count & $\$ 8.55$ per test & PU & 12/year & Ditto & $\$ 104.13$ \\
\hline $\begin{array}{l}\text { Cost after } \\
\text { year } 1\end{array}$ & $\$ 3,870.25$ & & & & & None-DRG cost & $\$ 3,870.25$ & & & & \\
\hline
\end{tabular}


Table 5.18: Costs of MI: the Most Conservative Situation

\begin{tabular}{|c|c|c|c|c|c|c|c|c|c|c|c|}
\hline Event & Year at the & vent & & & & Subsequent years & & & & & \\
\hline Event & $\begin{array}{l}\text { DRG Units } \\
\text { (Fatal) }\end{array}$ & $\begin{array}{l}\text { DRG Units } \\
\text { (Non-Fatal) }\end{array}$ & Source & $\begin{array}{l}\text { DRG Unit } \\
\text { price }\end{array}$ & Source & & Unit price & Source & dosage & Source & $\begin{array}{l}\text { Total } \\
\text { annual } \\
\text { costs }\end{array}$ \\
\hline MI & 2.8649 & 2.1921 & $\begin{array}{l}2018 \\
\text { DRG }\end{array}$ & $\$ 5,068.12$ & $\begin{array}{l}\text { MoH } \\
\text { Website }\end{array}$ & $\begin{array}{l}\text { Cilazapril with } \\
\text { hydrochlotothiazide }\end{array}$ & $\begin{array}{l}\text { 5mg per tab; } \\
\$ 10.8 \text { per } 100 \\
\text { tab }\end{array}$ & $\begin{array}{l}\text { PHARMAC } \\
\text { online } \\
\text { schedule }\end{array}$ & $5 \mathrm{mg} / \mathrm{d}$ & $\begin{array}{l}\text { Primary } \\
\text { care } \\
\text { handbook; } \\
\text { Medsafe }\end{array}$ & $\$ 39.42$ \\
\hline & & & & & & Metoprolol & $\begin{array}{l}50 \mathrm{mg} / \mathrm{tab} ; \\
\$ 5.66 / 100 \text { tabs }\end{array}$ & Ditto & $50 \mathrm{mg} / \mathrm{d}$ & Ditto & $\$ 20.66$ \\
\hline & & & & & & Statin & $\begin{array}{l}\text { 20mg/tab: } \\
\$ 9.99 / 500 \text { tabs }\end{array}$ & Ditto & $20 \mathrm{mg} / \mathrm{d}$ & Ditto & $\$ 7.29$ \\
\hline & & & & & & Marevan & $\begin{array}{l}1 \mathrm{mg} / \mathrm{tab} ; \\
\$ 7.6 / 100 \text { tabs }\end{array}$ & Ditto & $2 \mathrm{mg} / \mathrm{d}$ & Ditto & $\$ 55.48$ \\
\hline & & & & & & Aspirin & $\begin{array}{l}100 \mathrm{mg} \text { per tab; } \\
\$ 10.8 \text { per } 990\end{array}$ & Ditto & $100 \mathrm{mg} / \mathrm{d}$ & Ditto & $\$ 3.98$ \\
\hline & & & & & & Clopidogrel & $\begin{array}{l}75 \mathrm{mg} / \mathrm{tab} ; \\
5.44 / 84 \text { tabs }\end{array}$ & Ditto & $75 \mathrm{mg} / \mathrm{d}$ & Ditto & $\$ 23.64$ \\
\hline & & & & & & Cardiacology & $\$ 285.31 /$ time & PU & 1 visit/year & $\begin{array}{l}\text { Expert } \\
\text { opinion }\end{array}$ & $\$ 289.59$ \\
\hline DRG total cost & $\$ 14,737.58$ & $\$ 11,276.67$ & & & & Full blood count & $\$ 8.55 /$ test & PU & 1 test/year & Ditto & $\$ 8.68$ \\
\hline $\begin{array}{l}\text { Total cost at } \\
\text { the event }\end{array}$ & $\$ 14,737.58$ & $\$ 11,725.41$ & & & & $\begin{array}{l}\text { Total none-DRG } \\
\text { cost }\end{array}$ & $\$ 448.74$ & & & & \\
\hline $\begin{array}{l}\text { Annual cost } \\
\text { after year } 1\end{array}$ & $\$ 448.74$ & & & & & & & & & & \\
\hline
\end{tabular}


Table 5.19 Costs of the MI: Base Case

\begin{tabular}{|c|c|c|c|c|c|c|c|c|c|c|c|}
\hline \multirow{2}{*}{$\frac{\text { Event }}{\text { Event }}$} & \multicolumn{2}{|c|}{ Year at the event } & \multirow[b]{2}{*}{ Source } & \multirow[b]{2}{*}{$\begin{array}{l}\text { DRG Unit } \\
\text { price }\end{array}$} & \multirow[b]{2}{*}{ Source } & \multirow[t]{2}{*}{ Subsequent years } & \multirow[b]{2}{*}{ Unit price } & \multirow[b]{2}{*}{ Source } & \multirow[b]{2}{*}{ Dosage } & \multirow[b]{2}{*}{ Source } & \multirow[b]{2}{*}{$\begin{array}{l}\text { Total } \\
\text { annual } \\
\text { costs }\end{array}$} \\
\hline & $\begin{array}{l}\text { DRG Units } \\
\text { (Fatal) }\end{array}$ & $\begin{array}{l}\text { DRG Units } \\
\text { (Non-Fatal) }\end{array}$ & & & & & & & & & \\
\hline \multirow[t]{7}{*}{ MI } & 2.8649 & 2.1921 & $\begin{array}{l}2018 \\
\text { DRG }\end{array}$ & $\$ 5,068.12$ & $\begin{array}{l}\text { MoH } \\
\text { Website }\end{array}$ & $\begin{array}{l}\text { Cilazapril with } \\
\text { hydrochlotothiazide }\end{array}$ & $\begin{array}{l}\text { 5mg per tab; } \\
\$ 10.8 \text { per } 100 \\
\text { tab }\end{array}$ & $\begin{array}{l}\text { PHARMAC } \\
\text { online } \\
\text { schedule }\end{array}$ & $5 \mathrm{mg} / \mathrm{d}$ & $\begin{array}{l}\text { Primary care } \\
\text { handbook; } \\
\text { Medsafe }\end{array}$ & $\$ 39.42$ \\
\hline & & & & & & Metoprolol & $\begin{array}{l}50 \mathrm{mg} / \mathrm{tab} ; \\
\$ 5.66 / 100 \text { tabs }\end{array}$ & Ditto & $75 \mathrm{mg} / \mathrm{d}$ & Ditto & $\$ 30.99$ \\
\hline & & & & & & Statin & $\begin{array}{l}20 \mathrm{mg} / \mathrm{tab}: \\
\$ 9.99 / 500 \text { tabs }\end{array}$ & Ditto & $30 \mathrm{mg} / \mathrm{d}$ & Ditto & $\$ 10.94$ \\
\hline & & & & & & Marevan & $\begin{array}{l}1 \mathrm{mg} \text { per tab; } \\
\$ 7.6 \text { per } 100 \text { tab }\end{array}$ & Ditto & $4 \mathrm{mg} / \mathrm{d}$ & Ditto & $\$ 110.96$ \\
\hline & & & & & & Aspirin & $\begin{array}{l}\text { 100mg per tab; } \\
\$ 10.8 \text { per } 990\end{array}$ & Ditto & $100 \mathrm{mg} / \mathrm{d}$ & Ditto & $\$ 3.98$ \\
\hline & & & & & & Clopidogrel & $\begin{array}{l}75 \mathrm{mg} \text { per tab; } \\
5.44 \text { per } 84 \text { tab }\end{array}$ & Ditto & $75 \mathrm{mg} / \mathrm{d}$ & Ditto & $\$ 23.64$ \\
\hline & & & & & & Cardiacology & $\begin{array}{l}\$ 285.31 \quad \text { per } \\
\text { time }\end{array}$ & PU & $\begin{array}{l}3 \text { visits } \\
\text { per year }\end{array}$ & $\begin{array}{l}\text { Expert } \\
\text { opinion }\end{array}$ & $\$ 868.77$ \\
\hline $\begin{array}{l}\text { DRG total } \\
\text { cost }\end{array}$ & $\$ 14,737.58$ & $\$ 11,276.67$ & & & & Full blood count & $\$ 8.55 /$ test & PU & 3 tests & Ditto & $\$ 29.50$ \\
\hline $\begin{array}{l}\text { Cost at the } \\
\text { event year }\end{array}$ & $\$ 14,737.58$ & $\$ 12,394.87$ & & & & Total none-DRG cost & $\$ 1,118.2$ & & & & \\
\hline $\begin{array}{l}\text { Cost after } \\
\text { year } 1\end{array}$ & $\$ 1,118.2$ & & & & & & & & & & \\
\hline
\end{tabular}


Table 5.22: Costs of Renal Failure: The Most Serious Situation

\begin{tabular}{|c|c|c|c|c|c|c|c|c|c|c|c|}
\hline Event & Year at the ev & & & & & Subsequent & & & & & \\
\hline Event & $\begin{array}{l}\text { DRG Units } \\
\text { (Fatal) }\end{array}$ & $\begin{array}{l}\text { DRG Units } \\
\text { (Non-Fatal) }\end{array}$ & Source & $\begin{array}{l}\text { DRG Unit } \\
\text { price }\end{array}$ & Source & & Unit price & Source & dosage & Source & $\begin{array}{l}\text { Total annual } \\
\text { costs }\end{array}$ \\
\hline \multirow[t]{4}{*}{$\begin{array}{l}\text { Renal } \\
\text { failure }\end{array}$} & 3.69405 & & $\begin{array}{l}2018 \\
\text { DRG }\end{array}$ & $\$ 5,068.12$ & $\mathrm{MoH}$ & Dialysis & & & & $\begin{array}{l}2017 \quad \mathrm{NZ} \\
\text { nephrology } \\
11^{\text {th }} \text { annual } \\
\text { report }\end{array}$ & $\begin{array}{l}\$ 35,741.72 \\
(39,966.29 \\
\text { first year) }\end{array}$ \\
\hline & & & & & & Nephrologist & $\begin{array}{l}\$ 331.21 \text { per } \\
\text { time }\end{array}$ & PU & $\begin{array}{l}4 \text { visits } \\
\text { per year }\end{array}$ & $\begin{array}{l}\text { Expert } \\
\text { opinion }\end{array}$ & $\$ 1,324.84$ \\
\hline & & & & & & $\begin{array}{l}\text { Full blood } \\
\text { count }\end{array}$ & $\begin{array}{l}\$ 8.55 \text { per } \\
\text { test }\end{array}$ & PU & 4 tests & $\begin{array}{l}\text { Expert } \\
\text { opinion }\end{array}$ & $\$ 34.2$ \\
\hline & & & & & & Urine test & $\$ 34.07$ & PU & 4 tests & $\begin{array}{l}\text { Expert } \\
\text { opinion }\end{array}$ & $\$ 136,28$ \\
\hline $\begin{array}{l}\text { DRG total } \\
\text { cost }\end{array}$ & $\$ 19,002.89$ & $\$ 7,251.87$ & & & & None-DRG cost & $\begin{array}{l}\$ 37,237.04 \\
(\$ 41,461.61 \\
\text { first year) }\end{array}$ & & & & \\
\hline $\begin{array}{l}\text { Total cost } \\
\text { at the } \\
\text { event }\end{array}$ & $\$ 19,002.89$ & $\$ 48,713.87$ & & & & & & & & & \\
\hline $\begin{array}{l}\text { Annual } \\
\text { cost after } \\
\text { year } 1\end{array}$ & $\$ 37,237.04$ & & & & & & & & & & \\
\hline
\end{tabular}


Table 5.23: Costs of Renal Failure: the Most Conservative Situation

\begin{tabular}{|c|c|c|c|c|c|c|c|c|c|c|c|}
\hline Event & Year at the ev & & & & & Subsequent & & & & & \\
\hline Event & $\begin{array}{l}\text { DRG Units } \\
\text { (Fatal) }\end{array}$ & $\begin{array}{l}\text { DRG Units } \\
\text { (Non-Fatal) }\end{array}$ & Source & $\begin{array}{l}\text { DRG Unit } \\
\text { price }\end{array}$ & Source & & Unit price & Source & dosage & Source & $\begin{array}{l}\text { Total annual } \\
\text { costs }\end{array}$ \\
\hline \multirow[t]{4}{*}{$\begin{array}{l}\text { Renal } \\
\text { failure }\end{array}$} & 1.23135 & 0 & $\begin{array}{l}2018 \\
\text { DRG }\end{array}$ & $\$ 5,068.12$ & $\mathrm{MoH}$ & Dialysis & & & $\begin{array}{l}1 \text { per } \\
\text { week }\end{array}$ & $\begin{array}{l}2017 \quad \mathrm{NZ} \\
\text { nephrology } \\
11^{\text {th }} \text { annual } \\
\text { report }\end{array}$ & $\begin{array}{l}\$ 15,912.60 \\
(\$ 20,228.67 \\
\text { first year) }\end{array}$ \\
\hline & & & & & & Nephrologist & $\begin{array}{l}\$ 331.21 \text { per } \\
\text { time }\end{array}$ & PU & $\begin{array}{l}1 \text { visit } \\
\text { per year }\end{array}$ & $\begin{array}{l}\text { Expert } \\
\text { opinion }\end{array}$ & $\$ 331.21$ \\
\hline & & & & & & $\begin{array}{l}\text { Full blood } \\
\text { count }\end{array}$ & $\begin{array}{l}\$ 8.55 \text { per } \\
\text { test }\end{array}$ & PU & $\begin{array}{l}1 \text { test } \\
\text { per year }\end{array}$ & $\begin{array}{l}\text { Expert } \\
\text { opinion }\end{array}$ & $\$ 8.55$ \\
\hline & & & & & & Urine test & $\$ 34.07$ & PU & $\begin{array}{l}1 \text { test } \\
\text { per year }\end{array}$ & $\begin{array}{l}\text { Expert } \\
\text { opinion }\end{array}$ & $\$ 34.07$ \\
\hline $\begin{array}{l}\text { DRG total } \\
\text { cost }\end{array}$ & $\$ 12,668.48$ & & & & & None-DRG cost & $\begin{array}{l}\$ 16,286.43 \\
(\$ 20,602.5 \\
\text { first year) }\end{array}$ & & & & \\
\hline $\begin{array}{l}\text { Total cost } \\
\text { at the } \\
\text { event }\end{array}$ & & $\$ 20,602.5$ & & & & & & & & & \\
\hline $\begin{array}{l}\text { Annual } \\
\text { cost after } \\
\text { year } 1\end{array}$ & & $\$ 16,286.43$ & & & & & & & & & \\
\hline
\end{tabular}


Table 5.24: Costs of Renal Failure: the Base Case

\begin{tabular}{|c|c|c|c|c|c|c|c|c|c|c|c|}
\hline \multirow{2}{*}{$\begin{array}{l}\text { Event } \\
\text { Event }\end{array}$} & \multicolumn{2}{|c|}{ Year at the event } & \multirow[b]{2}{*}{ Source } & \multirow[b]{2}{*}{$\begin{array}{l}\text { DRG Unit } \\
\text { price }\end{array}$} & \multirow[b]{2}{*}{ Source } & \multirow{2}{*}{$\begin{array}{l}\text { Subsequent } \\
\text { years }\end{array}$} & \multirow[b]{2}{*}{ Unit price } & \multirow[b]{2}{*}{ Source } & \multirow[b]{2}{*}{ dosage } & \multirow[b]{2}{*}{ Source } & \multirow[b]{2}{*}{$\begin{array}{l}\text { Total annual } \\
\text { costs }\end{array}$} \\
\hline & $\begin{array}{l}\text { DRG Units } \\
\text { (Fatal) }\end{array}$ & $\begin{array}{l}\text { DRG Units } \\
\text { (Non-Fatal) }\end{array}$ & & & & & & & & & \\
\hline \multirow[t]{4}{*}{$\begin{array}{l}\text { Renal } \\
\text { failure }\end{array}$} & 1.23135 & 0 & $\begin{array}{l}2018 \\
\text { DRG }\end{array}$ & $\$ 5,068.12$ & $\mathrm{MoH}$ & Dialysis & & & $\begin{array}{l}2 \text { per } \\
\text { week }\end{array}$ & $\begin{array}{l}2017 \quad \mathrm{NZ} \\
\text { nephrology } \\
11^{\text {th }} \text { annual } \\
\text { report }\end{array}$ & $\begin{array}{l}\$ 25,827.16 \\
(\$ 30,153.23 \\
\text { first year })\end{array}$ \\
\hline & & & & & & Nephrologist & $\begin{array}{l}\$ 331.21 \text { per } \\
\text { time }\end{array}$ & PU & $\begin{array}{l}2 \text { visit } \\
\text { per year }\end{array}$ & $\begin{array}{l}\text { Expert } \\
\text { opinion }\end{array}$ & $\$ 662.42$ \\
\hline & & & & & & $\begin{array}{l}\text { Full blood } \\
\text { count }\end{array}$ & $\begin{array}{l}\$ 8.55 \text { per } \\
\text { test }\end{array}$ & PU & $\begin{array}{l}2 \text { tests } \\
\text { per year }\end{array}$ & $\begin{array}{l}\text { Expert } \\
\text { opinion }\end{array}$ & $\$ 17.10$ \\
\hline & & & & & & Urine test & $\$ 34.07$ & $\mathrm{PU}$ & $\begin{array}{l}2 \text { test } \\
\text { per year }\end{array}$ & $\begin{array}{l}\text { Expert } \\
\text { opinion }\end{array}$ & $\$ 68.14$ \\
\hline $\begin{array}{l}\text { DRG total } \\
\text { cost }\end{array}$ & $\$ 12,668.48$ & & & & & None-DRG cost & $\begin{array}{l}\$ 26,574.82 \\
(30,900.89 \\
\text { first year) }\end{array}$ & & & & \\
\hline $\begin{array}{l}\text { Total cost } \\
\text { at the } \\
\text { event }\end{array}$ & & $\$ 30,900.89$ & & & & & & & & & \\
\hline $\begin{array}{l}\text { Annual } \\
\text { cost after } \\
\text { year } 1\end{array}$ & & $\$ 26,574.82$ & & & & & & & & & \\
\hline
\end{tabular}


Table 5.25 Costs of Glycaemia Control

\begin{tabular}{|c|c|c|c|c|c|c|}
\hline Drug cost & Unit Price & Source & Dosage & Source & $\begin{array}{l}\text { Total cost per } \\
\text { year }\end{array}$ & $\begin{array}{l}\text { Proportion } \\
\text { (according to } \\
\text { Scott et a.'s) }\end{array}$ \\
\hline Metformin & 1,000mg per tab; $\$ 40$ per 60 & $\begin{array}{l}\text { Pharmac online } \\
\text { schedule }\end{array}$ & $2 \mathrm{~g} / \mathrm{d}$ & Medsafe & $\$ 15.42$ & \multirow[t]{4}{*}{$33.09 \%$} \\
\hline \multicolumn{6}{|l|}{ None drug cost } & \\
\hline Pharmacy services & $\$ 5.44$ per service & $\mathrm{MoH}$ & 4 times/year & Expert opinion & $\$ 22.09$ & \\
\hline Total & & & & & $\$ 37.51$ & \\
\hline Metformin with Vildogliptin & 1,000mg per tab; $\$ 40$ per 60 & $\begin{array}{l}\text { Pharmac online } \\
\text { schedule }\end{array}$ & $2 \mathrm{~g} / \mathrm{d}$ & Medsafe & $\$ 486.66$ & \multirow[t]{5}{*}{$31.42 \%$} \\
\hline \multicolumn{6}{|l|}{ None drug cost } & \\
\hline Pharmacy services & $\$ 5.44$ per service & $\mathrm{MoH}$ & 4 times/year & Expert opinion & $\$ 22.09$ & \\
\hline Total & & & & & $\$ 508.75$ & \\
\hline Drug cost & Unit Price & Source & Dosage & Source & $\begin{array}{l}\text { Total cost per } \\
\text { year }\end{array}$ & \\
\hline Metformin with Vildogliptin & 1,000mg per tab; $\$ 40$ per 60 & $\begin{array}{l}\text { Pharmac online } \\
\text { schedule }\end{array}$ & $2 \mathrm{~g} / \mathrm{d}$ & Medsafe & $\$ 486.66$ & \multirow[t]{4}{*}{$28.42 \%$} \\
\hline Insulin pin & $\$ 94.5$ each & $\begin{array}{l}\text { Pharmac online } \\
\text { schedule }\end{array}$ & 1 pen / month & Expert opinion & $\$ 1,134$ & \\
\hline Pharmacy services & $\$ 5.44$ per service & $\mathrm{MoH}$ & 4 times/year & Expert opinion & $\$ 22.09$ & \\
\hline Total & & & & & $\$ 1,642.75$ & \\
\hline Drug cost & Unit Price & Source & Dosage & Source & $\begin{array}{l}\text { Total cost per } \\
\text { year }\end{array}$ & \multirow[t]{2}{*}{$4.59 \%$} \\
\hline Insulin pin & $\$ 94.5$ each & $\begin{array}{l}\text { Pharmac online } \\
\text { schedule }\end{array}$ & 1 pen / month & Expert opinion & $\$ 1,134$ & \\
\hline
\end{tabular}




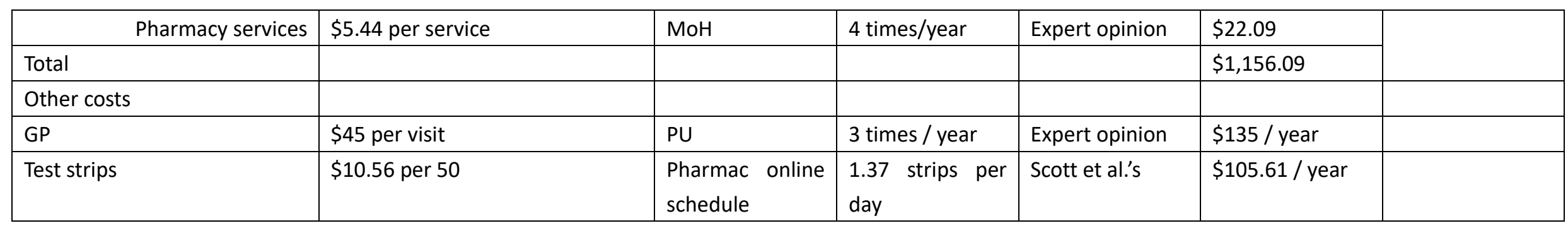

Table 5.20 Costs of Blindness

\begin{tabular}{|l|l|l|l|l|l|}
\hline Event & Cost at the event year \& subsequent years & Average salary & Source & Care hours per week & Source \\
\hline Blindness & Care Costs & $\$ 18,229.14$ & $\$ 23.75 /$ hour & $\begin{array}{l}\text { Stats } \\
\text { NZ }\end{array}$ & $\begin{array}{l}14.72 \mathrm{~h} \\
\text { Green et al's }\end{array}$ \\
\hline & Ophthalmologist & $(\$ 228.87+\$ 173.81) * 1.015=\$ 408.72$ (first year only) & & & \\
\hline & Taxi Costs & $\$ 23.43 /$ week $(\$ 1,551.69$ after adjusted by inflation rates) & & & \\
\hline
\end{tabular}

Figure 5.8 Stroke Rate by Year Predicted by the UKPDS Model

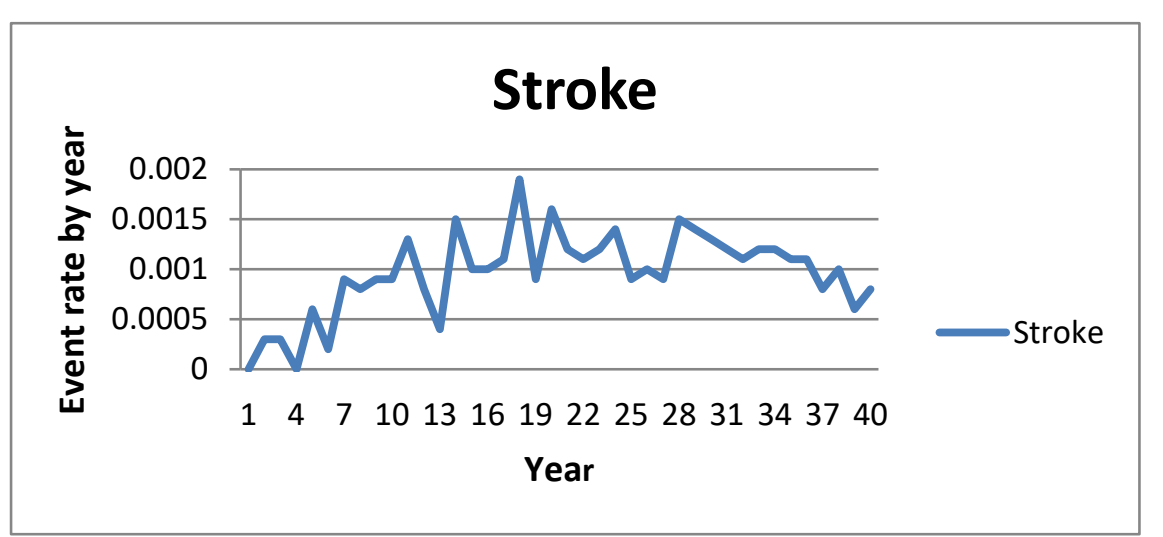

\section{Figure 5.9 IHD Rate by Year Predicted by the UKPDS Model}

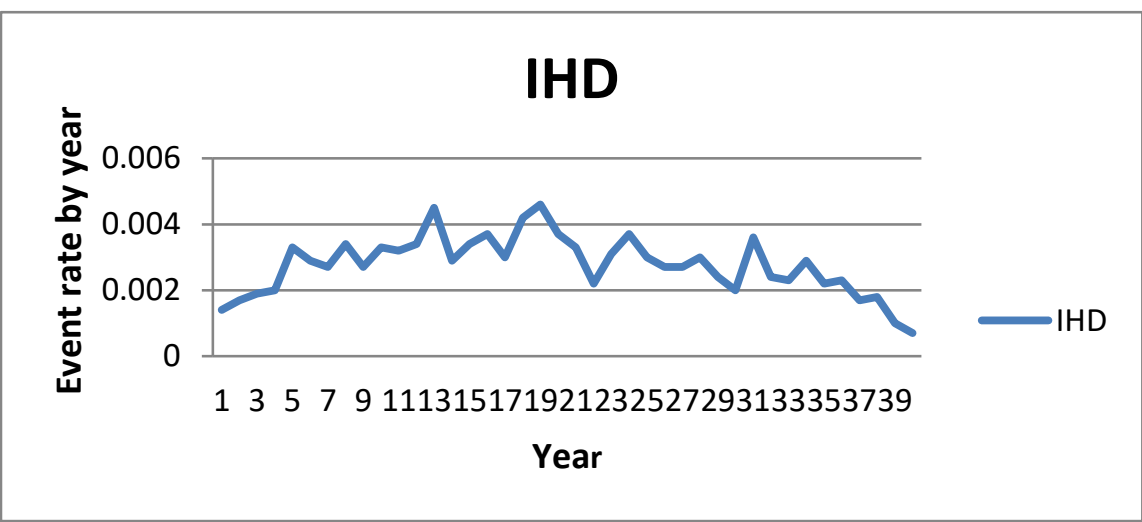


Table 5.26: Diabetes Costs in Absence of Complications

\begin{tabular}{|c|c|c|c|}
\hline & & Weights / Times & Costs \\
\hline \multirow[t]{8}{*}{ Base Case } & Metformin alone & $33.09 \%$ & $\$ 37.51$ \\
\hline & Metformin + Vildogaliptin & $31.42 \%$ & $\$ 508.75$ \\
\hline & Metformin + Vildogaliptin + Insulin & $28.42 \%$ & $\$ 1,642.75$ \\
\hline & Insulin alone & $4.59 \%$ & $\$ 1,156.09$ \\
\hline & GP visits & 3 times per year & \$45/visit; \$135 /year \\
\hline & Test strips & 1.37 strips per day & $\$ 10.56$ per $50 ; \$ 105.61 /$ year \\
\hline & Diabetes Education & & $\$ 273.07$ (One off); $\$ 273.07 / 30$ years $=\$ 9.10 /$ year \\
\hline & In Total & & $\$ 941.91$ \\
\hline \multirow{5}{*}{$\begin{array}{l}\text { The Most Conservative } \\
\text { Situation }\end{array}$} & Metformin alone & & $\$ 37.51$ \\
\hline & GP visits & once per year & $\$ 45$ \\
\hline & Test strips & 1 strips per day & $\$ 77.09$ / year \\
\hline & Diabetes Education & & $\$ 9.10$ / year \\
\hline & In Total & & $\$ 168.7$ \\
\hline \multirow{6}{*}{$\begin{array}{l}\text { The Most Serious } \\
\text { Situation }\end{array}$} & Metformin + Vildogaliptin + Insulin & & $\$ 1,642.75$ \\
\hline & & & \\
\hline & GP visits & Once per month & $\$ 540 /$ year \\
\hline & Test strips & 3 times / day & $\$ 231.26$ \\
\hline & Diabetes Education & & $\$ 9.10$ / year \\
\hline & In Total & & $\$ 2,423.11$ \\
\hline
\end{tabular}


Table 5.27: DRGs Used in Costing

\begin{tabular}{|c|c|c|}
\hline Disease & Activity & $\mathrm{DRG} / \mathrm{PU}$ \\
\hline \multirow[t]{6}{*}{ Stroke } & Hospitalization & DRG B70A: Stroke \& Other Cerebrovascular Disorders W Catastrophic CC) \\
\hline & & DRG B70B (Stroke \& Other Cerebrovascular Disorders W Severe CC) \\
\hline & & DRG B70C (Stroke \& Other Cerebrovascular Disorders W/O Catastrophic or Severe CC) \\
\hline & & DRG B70D: Stroke \& Other Cerebrovascular Disorders, Died/Trans Acute Facility $<5$ Days \\
\hline & Rehabilitation Outpatient & DRG Z60Z: Rehabilitation \\
\hline & & $\begin{array}{l}\text { Outpatient - clinics (Assessment, treatment and/or rehabilitation in a non-home setting by a health } \\
\text { professional. PCU 18/19). }\end{array}$ \\
\hline \multirow[t]{2}{*}{ MI } & Hospitalization & $\begin{array}{l}\text { DRG F10B: Interventional Coronary Procedures Admitted for AMI Without Catastrophic CC } \\
\text { (complications) }\end{array}$ \\
\hline & & DRG F10A: Interventional Coronary Procedures Admitted for AMI With Catastrophic CC (complications) \\
\hline \multirow[t]{3}{*}{ Heart Failure } & Hospitalization & DRG F62A: Heart failure and shock with catastrophic CC \\
\hline & & DRG62B: Heart failure and shock without catastrophic CC \\
\hline & & DRG62C: Heart Failure and Shock, Died or Transferred to Acute Facility $<5$ Days \\
\hline \multirow[t]{3}{*}{ Kidney failure } & Hospitalization & DRG L60A: Kidney failure and shock w catastrophic CC \\
\hline & & DRG L60B: Kidney failure and shock w severe CC \\
\hline & & DRG L60C: Kidney failure and shock w/o catastrophic CC \\
\hline \multirow[t]{2}{*}{ Amputation } & Hospitalization & DRG F11B: Amputation, Except Upper Limb and Toe, for Circulatory Disorders W/O Cat CC \\
\hline & & DRG F11A: Amputation, Except Upper Limb and Toe, for Circulatory Disorders W Cat CC \\
\hline
\end{tabular}




\section{Calculation of Probabilities}

Convert the probability of diabetes in one-year to probability in ten-year:

Base case:

One - year probability $=0.001628$,

Assuming incidence rate is constant in the 10 years, and then,

$$
\text { Rate }=-\ln (1-0.001628)=0.001629,
$$

So, the probability in 10 years is:

$$
\text { Ten }- \text { year probability }=1-\exp (-0.001629 * 10)=0.016161 .
$$

Similarly, in the sensitivity analysis, when one - year probability $=0.00145$,

$$
\text { Rate }=-\ln (1-0.00145)=0.001451 \text {, }
$$

So, the probability in 10 years is:

$$
\text { Ten - year probability }=1-\exp (-0.001451 * 10)=0.01441 .
$$

Similarly, in the sensitivity analysis, when one - year probability $=0.0008$,

$$
\text { Rate }=-\ln (1-0.0008)=0.0008,
$$

So, the probability in 10 years is:

Ten - year probability $=1-\exp (-0.0008 * 10)=0.007$

(2) Convert the probability of IHD in one-year to ten-year

Base case:

$$
\text { One - year probability }=0.00411 \text {, }
$$

Assuming incidence rate is constant in the 10 years, and then,

$$
\text { Rate }=-\ln (1-0.00411)=0.00412,
$$

So, the probability in 10 years is:

$$
\text { Ten }- \text { year probability }=1-\exp (-0.00412 * 10)=0.04035 \text {. }
$$

Similarly, in the sensitivity analysis, when one-year probability $=0.0062$, the probability in 10 years is: 0.0603 ; when one-year probability $=0.0028$, the probability in 10 years is: 0.0275 
(3) Convert the probability of stroke in one-year to probability in ten-year Base case:

One - year probability $=0.00185$,

Assuming incidence rate is constant in the 10 years, and then,

$$
\text { Rate }=-\ln (1-0.00411)=0.00185,
$$

So, the probability in 10 years is:

Ten - year probability $=1-\exp (-0.00185 * 10)=0.01835$. 


\section{Calculation in Sensitivity Analysis for Costs of Stroke \& IHD}

(a) Stroke

In the sensitivity analysis, if the costs of a fatal stroke are changed to $\$ 1,735.01$ in the first year, and the costs of a non-fatal change to $\$ 5,162.89$ and $\$ 158.05$ in the first year and subsequent years respectively, then the lifetime costs of a stroke will decrease to $\$ 5,527.41$ in the most conservative scenario. In this case, the average cost per litre would decrease to $\$ 0.009$.

If the costs of a fatal stroke increase to $\$ 5,205.03$ in the first year, and the costs of a non-fatal stroke change to $\$ 52,472.21$ and $\$ 34,946.13$ in the first year and subsequent years respectively, then the lifetime costs of a stroke will reach $\$ 221,904.48$ in the most serious situation. As a result, the average cost per litre would be $\$ 266.29 /(70.71 * 10)=\$ 0.377$.

If we assumed that SSB consumption for an ordinary person (a light-to-medium drinker) was 68.36 litres, then the harm associated with a stroke from SSB consumption was approximately $\$ 104.66 /(68.36 * 10)=\$ 0.153$ per litre.

If the probability of survival for a stroke survivor after year one is estimated from the information of the ischaemic stroke patients only, then the lifetime total costs after discounting would change to approximately $\$ 128,813.87$. In this case, the light-drinkers were expected to cost $\$ 128,813.87 *(0.0183-0.0171)=\$ 154.58$ more, compared with non-drinkers; and the harm associated with a stroke from SSB consumption would correspondingly change to $\$ 154.58 /(70.71 * 10)=\$ 0.219$ per litre.

(b) IHD

In the most optimistic situation, the annual costs in the first year and subsequent years are $\$ 7,494.07$ and $\$ 425.10$ respectively. The lifetime costs after discounting would correspondingly change to $\$ 8,888.54$ in this case. As a result, the harm associated with an IHD from SSB consumption would correspondingly change to $\$ 0.009$ per litre.

In the most severe case, the annual costs in the first year and subsequent years are $\$ 16,861.65$ and $\$ 1,484.11$, respectively. In this case, the lifetime costs after 
discounting would correspondingly change to $\$ 24,288.10$, and the harm associated with a heart disease from SSB consumption would correspondingly increase to $\$ 0.024$ per litre.

If we assumed that the SSB consumption for an ordinary person (a light-to-medium drinker) was 68.36 litres, then the harm associated with an IHD from SSB consumption would be approximately $\$ 11.91 /(68.36 * 10)=\$ 0.017$ per litre.

If the incidence rate was changed to 0.0062 , then the probability of developing an IHD for a light drinker would be approximately 0.0603 in 10 years. Given the hazard ratio between light drinkers and non-drinkers (1.02), the probability of developing an IHD for a non-drinker would be $0.0603 / 1.02=0.0591$ in 10 years. Therefore, the light-drinkers were expected to cost $\$ 17,018.60 *(0.0603-0.0591)=$ \$20.42 more than non-drinkers. Given the SSB consumption per person per year $(70.71 \mathrm{~L})$, the average cost per litre would be approximately $\$ 20.42 /(70.71 * 10)=$ $\$ 0.029$.

If the incidence rate was changed to 0.0028 , then the probability of developing an IHD for a light drinker would be approximately 0.0275 in 10 years. Given the hazard ratio between light drinkers and non-drinkers (1.02), the probability of developing an IHD for a non - drinker would be $0.0275 / 1.02=0.0270$ in 10 years. Therefore, the light-drinkers were expected to cost $\$ 17,018.60 *(0.0275-0.0270)=$ \$8.51 more than with non - drinkers. Given the SSB consumption per person per year $(70.71 \mathrm{~L})$, the average cost per litre would be approximately $\$ 8.51 /(70.71 * 10)=$ $\$ 0.012$.

If the probability of survival for an IHD survivor was derived from the data of the patients in the Invercargill hospital, then the lifetime costs after discounting would change to approximately $\$ 15,957.03$. In this case, the light-drinkers would be expected to cost $\$ 15,957.03 * 0.0007=\$ 11.17$ more than with non-drinkers; and the harm associated with an IHD from SSB consumption would correspondingly change to $\$ 11.17 /(70.71 * 10)=\$ 0.016$ per litre.

(c) Diabetes 
If we assume the diabetes incidence in 2019 was 0.0008 , as predicted using the linear regression method, then the diabetes cost per litre would decrease to $\$ 0.023$. If we assume the incidence in 2019 is the same as in 2018, the diabetes cost per litre would decrease to $\$ 0.040$.

If diabetic patients are on metformin monotherapy, have one GP visit per year, and one strip test per day, the total lifetime costs would go down to $\$ 17,510.92$. In this case, the diabetes cost per litre would decrease to $\$ 0.026$. In the most severe case, costs in absence of diabetic complications are $\$ 2,423.11$ per year, and total lifetime costs are $\$ 54,900.98$. In this case, the diabetes cost per litre increases to $\$ 0.082$.

If the SSB consumption change to 68.36L per person per year, then the diabetes cost per litre would be $\$ 32.02 /(68.36 * 10)=\$ 0.047$. 


\section{Appendix V: Information for Chapter 6}

Table 6.1: Results of Simulation (full table)

\begin{tabular}{|c|c|c|c|c|c|c|c|c|c|c|c|}
\hline \multirow{2}{*}{$\begin{array}{l}\text { Calibrated } \\
\text { with estimates } \\
\text { from }\end{array}$} & \multirow{2}{*}{$\begin{array}{c}\text { Category of } \\
\text { Tax }\end{array}$} & \multirow{2}{*}{$\begin{array}{l}\text { Initial } \\
\text { Welfare }\end{array}$} & \multicolumn{3}{|c|}{ Per Litre Tax } & \multicolumn{3}{|c|}{ Ad Valorem Tax } & \multicolumn{3}{|c|}{ Calorie Tax } \\
\hline & & & $\begin{array}{l}\text { Scale } \\
\text { Factor } \\
\text { (Socially - } \\
\text { Optimal } \\
\text { Tax Level) }\end{array}$ & $\begin{array}{l}\text { Welfare } \\
\text { Post-Tax }\end{array}$ & $\begin{array}{l}\text { Change in } \\
\text { Welfare }\end{array}$ & $\begin{array}{l}\text { Scale } \\
\text { Factor } \\
\text { (Socially - } \\
\text { Optimal } \\
\text { Tax Level) }\end{array}$ & $\begin{array}{l}\text { Welfare } \\
\text { Post-Tax }\end{array}$ & $\begin{array}{l}\text { Change } \\
\text { in } \\
\text { Welfare }\end{array}$ & $\begin{array}{l}\text { Scale } \\
\text { Factor } \\
\text { (Socially - } \\
\text { Optimal } \\
\text { Tax Level }\end{array}$ & $\begin{array}{l}\text { Welfare } \\
\text { Post-Tax }\end{array}$ & $\begin{array}{l}\text { Change } \\
\text { Welfare }\end{array}$ \\
\hline & $\begin{array}{l}\text { Beverage } \\
\text { Taxes }\end{array}$ & -465.57 & $\begin{array}{l}203.23 \\
(6.50)\end{array}$ & 497.61 & 963.18 & $\begin{array}{l}37.78 \\
(1.21)\end{array}$ & 168.72 & 634.29 & $\begin{array}{l}1.0000 \\
(0.032)\end{array}$ & 567.09 & $1,032.66 * *$ \\
\hline $\begin{array}{l}\text { Ni Mhurchu et } \\
\text { al. } \\
(\mathrm{H}=\mathrm{NZ} \$ 0.0006)\end{array}$ & SSB Taxes & 70.726 & $\begin{array}{l}228.50 \\
(0.14)\end{array}$ & 71.087 & $0.361^{*}$ & $\begin{array}{l}65.27 \\
(0.04)\end{array}$ & 71.085 & 0.359 & $\begin{array}{l}1.0002 \\
(0.0006)\end{array}$ & 71.086 & 0.360 \\
\hline $\begin{array}{l}\text { Sharma et al. } \\
\text { (H=NZ\$0.0006 } \\
=\text { AU\$0.0006) }\end{array}$ & SSB Taxes & 111.80 & $\begin{array}{l}234.93 \\
(0.13)\end{array}$ & 111.88 & 0.085 & $\begin{array}{l}101.83 \\
(0.06)\end{array}$ & 111.81 & 0.016 & $\begin{array}{l}0.5888 \\
(0.0003)\end{array}$ & 111.89 & $0.089 *$ \\
\hline
\end{tabular}




\begin{tabular}{|c|c|c|c|c|c|c|c|c|c|c|c|}
\hline & $\begin{array}{l}\text { Beverage } \\
\text { Taxes }\end{array}$ & 111.80 & $\begin{array}{l}118.89 \\
(0.07)\end{array}$ & 112.05 & 0.249 & $\begin{array}{l}165.76 \\
(0.09)\end{array}$ & 112.14 & 0.342 & $\begin{array}{l}1.00 \\
(0.0006)\end{array}$ & 112.40 & $0.598 * *$ \\
\hline \multirow{2}{*}{$\begin{array}{l}\text { Dharmasena \& } \\
\text { Capps's } \\
(\mathbf{H}=\text { NZ\$0.0006 } \\
=\text { US\$0.0004) }\end{array}$} & SSB Taxes & 1.413 & $\begin{array}{l}241.86 \\
(0.10)\end{array}$ & 1.522 & 0.109 & $\begin{array}{l}627.59 \\
(0.25)\end{array}$ & 1.522 & 0.109 & $\begin{array}{l}0.60 \\
(0.0002)\end{array}$ & 1.523 & $0.110^{*}$ \\
\hline & $\begin{array}{l}\text { Beverage } \\
\text { Taxes }\end{array}$ & 1.413 & $\begin{array}{l}204.84 \\
(0.08)\end{array}$ & 1.627 & 0.214 & $\begin{array}{l}823.67 \\
(0.33)\end{array}$ & 1.768 & 0.355 & $\begin{array}{l}1.00 \\
(0.0004)\end{array}$ & 1.795 & $0.382 * *$ \\
\hline
\end{tabular}

(H=NZ\$0.0006

$=$ US\$0.0004)

*The highest increase in welfare in a certain category of tax; ** the highest increase in welfare in a certain category of all taxes

Table 6.2: Simulated Effects of Taxes Using Estimates from Ni Mhurchu et al.'s.

\begin{tabular}{|c|c|c|c|c|c|c|c|c|c|c|}
\hline Tax Type & $\begin{array}{c}\text { Welfare } \\
\text { Before-Tax }\end{array}$ & $\begin{array}{l}\text { Welfare } \\
\text { Post-Tax }\end{array}$ & $\begin{array}{c}\text { Change } \\
\text { in } \\
\text { Welfare }\end{array}$ & $\begin{array}{c}\text { Utility } \\
\text { Before-Tax }\end{array}$ & $\begin{array}{c}\text { Utility } \\
\text { After-Tax }\end{array}$ & $\begin{array}{c}\text { Change } \\
\text { in } \\
\text { Utility }\end{array}$ & $\begin{array}{c}\text { Tax } \\
\text { Revenue }\end{array}$ & $\begin{array}{c}\text { Deadweight Loss } \\
\text { (Change in Utility } \\
\text { Plus Tax } \\
\text { Revenue) }\end{array}$ & $\begin{array}{c}\text { Harm } \\
\text { Reduced }\end{array}$ & $\begin{array}{c}\text { Correlatior } \\
\text { with } \\
\text { Calories }\end{array}$ \\
\hline \multicolumn{11}{|l|}{ SSB Taxes } \\
\hline Ad Valorem Tax & 70.726 & 71.085 & 0.359 & 80.974 & 72.901 & -8.073 & 7.712 & -0.361 & 0.720 & 0.958 \\
\hline Sugar Tax & 70.726 & 71.086 & 0.360 & 80.974 & 72.379 & -8.595 & 8.233 & -0.362 & 0.722 & 0.998 \\
\hline Volume Tax & 70.726 & 71.087 & 0.361 & 80.974 & 72.028 & -8.946 & 8.577 & -0.369 & 0.730 & 0.970 \\
\hline \multicolumn{11}{|l|}{ Beverage Taxes } \\
\hline Ad Valorem Tax & 70.726 & 70.949 & 0.223 & 80.974 & 68.022 & -12.952 & 12.729 & -0.223 & 0.446 & -0.699 \\
\hline Sugar Tax & 70.726 & 71.089 & 0.363 & 80.974 & 71.09 & -9.884 & 9.520 & -0.364 & 0.727 & 1.000 \\
\hline Volume Tax & 70.726 & 71.064 & 0.338 & 80.974 & 68.222 & -12.752 & 12.424 & -0.328 & 0.666 & 0.958 \\
\hline
\end{tabular}


Table 6.3: Simulated Effects of Taxes Using Estimates from Sharma et al.'s

\begin{tabular}{|c|c|c|c|c|c|c|c|c|c|c|}
\hline Tax Type & $\begin{array}{c}\text { Welfare } \\
\text { Before-Tax }\end{array}$ & $\begin{array}{l}\text { Welfare } \\
\text { Post-Tax }\end{array}$ & $\begin{array}{l}\text { Change } \\
\text { in } \\
\text { Welfare }\end{array}$ & $\begin{array}{c}\text { Utility } \\
\text { Before-Tax }\end{array}$ & $\begin{array}{c}\text { Utility } \\
\text { After-Tax }\end{array}$ & $\begin{array}{c}\text { Change } \\
\text { in } \\
\text { Utility }\end{array}$ & $\begin{array}{c}\text { Tax } \\
\text { Revenue }\end{array}$ & $\begin{array}{c}\text { Deadweight Loss } \\
\text { (Change in Utility } \\
\text { Plus Tax } \\
\text { Revenue) }\end{array}$ & $\begin{array}{c}\text { Harm } \\
\text { Reduced }\end{array}$ & $\begin{array}{c}\text { Correlation } \\
\text { with } \\
\text { Calories }\end{array}$ \\
\hline Sugar Tax & 111.799 & 111.888 & 0.089 & 118.846 & 117.630 & -1.216 & 1.1260 & -0.090 & 0.179 & 0.453 \\
\hline Volume Tax & 111.799 & 111.884 & 0.085 & 118.846 & 117.673 & -1.174 & 1.090 & -0.084 & 0.169 & 0.440 \\
\hline Ad Valorem Tax & 111.799 & 111.815 & 0.016 & 118.846 & 118.169 & -0.677 & 0.662 & -0.015 & 0.031 & 0.428 \\
\hline Sugar Tax & 111.799 & 112.396 & 0.598 & 118.846 & 112.487 & -6.360 & 5.780 & -0.579 & 1.177 & 1.000 \\
\hline Volume Tax & 111.799 & 112.048 & 0.249 & 118.846 & 114.605 & -4.242 & 4.002 & -0.240 & 0.490 & 0.394 \\
\hline Ad Valorem Tax & 111.799 & 112.141 & 0.342 & 118.846 & 112.664 & -6.183 & 5.831 & -0.352 & 0.694 & 0.683 \\
\hline Tax Type & $\begin{array}{c}\text { Welfare } \\
\text { Before-Tax }\end{array}$ & $\begin{array}{l}\text { Welfare } \\
\text { Post-Tax }\end{array}$ & $\begin{array}{c}\text { Change } \\
\text { in } \\
\text { Welfare }\end{array}$ & $\begin{array}{c}\text { Utility } \\
\text { Before-Tax }\end{array}$ & $\begin{array}{c}\text { Utility } \\
\text { After-Tax }\end{array}$ & $\begin{array}{c}\text { Change } \\
\text { in } \\
\text { Utility }\end{array}$ & $\begin{array}{c}\text { Tax } \\
\text { Revenue }\end{array}$ & $\begin{array}{l}\text { Deadweight Loss } \\
\text { (Change in Utility } \\
\text { + Tax Revenue) }\end{array}$ & $\begin{array}{c}\text { Harm } \\
\text { Reduced }\end{array}$ & $\begin{array}{c}\text { Correlation } \\
\text { with } \\
\text { Calories } \\
\end{array}$ \\
\hline Sugar Tax & 1.413 & 1.523 & 0.010 & 3.236 & 2.898 & -0.338 & 0.231 & -0.107 & 0.217 & 0.451 \\
\hline Volume Tax & 1.413 & 1.522 & 0.009 & 3.236 & 2.920 & -0.316 & 0.209 & -0.107 & 0.216 & 0.438 \\
\hline Ad Valorem Tax & 1.413 & 1.522 & 0.009 & 3.236 & 2.910 & -0.326 & 0.224 & -0.102 & 0.211 & 0.419 \\
\hline \multicolumn{11}{|l|}{ Beverage Taxes } \\
\hline Sugar Tax & 1.413 & 1.795 & 0.382 & 3.236 & 1.798 & -1.438 & 1.058 & -0.380 & 0.762 & 1.000 \\
\hline Volume Tax & 1.413 & 1.627 & 0.214 & 3.236 & 2.013 & -1.223 & 1.018 & -0.205 & 0.419 & 0.394 \\
\hline Ad Valorem Tax & 1.413 & 1.768 & 0.355 & 3.236 & 1.568 & -1.668 & 1.309 & -0.359 & 0.714 & 0.771 \\
\hline
\end{tabular}


Table 6.5: Post-Tax Consumption Using Estimates from Sharma et al.'s.

\begin{tabular}{lcc}
\hline Beverage & Ad Valorem SSB taxes & Beverage Taxes by Calories \\
\hline Regular soft drinks & 5.57 & 6.16 \\
Cordial & 3.00 & 1.73 \\
Fruit drink & 0.60 & 0.46 \\
Diet soft drinks & 5.34 & 5.81 \\
Bottled water & 0.91 & 0.89 \\
Fruit juice & 3.84 & 3.42 \\
High-fat milk & 6.45 & 4.62 \\
Low-fat milk & 7.11 & 5.90 \\
Tea & 14.77 & 13.06 \\
Coffee & 21.29 & 19.25 \\
\hline
\end{tabular}




\section{References}

Adair LS, Kumar Arora N, Azizi F, Baur L, et al. (2016). Consideration of the evidence on childhood obesity for the Commission on Ending Childhood Obesity: Report of the Adhoc Working Group on Science and Evidence for Ending Childhood Obesity. Geneva, Switzerland: World Health Organization.

Allcott, Hunt, Benjamin B Lockwood, and Dmitry Taubinsky. (2019). "Regressive Sin Taxes, with an Application to the Optimal Soda Tax." Quarterly Journal of Economics 134 (3):1557-1626.

Andreyeva, T., Chaloupka, F., \& Brownell, K. (2011). Estimating the potential of taxes on sugar-sweetened beverages to reduce consumption and generate revenue. Preventive Medicine, 52(6), 413-416. doi: 10.1016/j.ypmed.2011.03.013

Annemarei Ranta. (2018). Projected stroke volumes to provide a 10 year direction for new Zealand stroke services. New Zealand Medical Association (NZMA) 22 June 2018, Vol 131, No 1477, ISSN 1175-8716.

Azaïs-Braesco, V., Sluik, D., Maillot, M., Kok, F. and Moreno, L., 2017. A review of total \& added sugar intakes and dietary sources in Europe. Nutrition Journal, 16(1).

Backholer, K., Sarink, D., Beauchamp, A., Keating, C., Loh, V., Ball, K., Martin, J. and Peeters, A. (2016). The impact of a tax on sugar-sweetened beverages according to socio-economic position: a systematic review of the evidence. Public Health Nutrition, 19(17), pp.3070-3084.

Baker MG, Goodyear R, Telfar Barnard L, Howden-Chapman P. (2012). The Distribution of Household Crowding in New Zealand: An analysis based on 1991 to 2006 Census data. Wellington: He Kainga Oranga/Housing and Health Research Programme, University of Otago.

Barquera, S., Hernandez-Barrera, L., Tolentino, M., Espinosa, J., Ng, S., Rivera, J., \& 
Popkin, B. (2008). Energy Intake from Beverages Is Increasing among Mexican Adolescents and Adults. The Journal Of Nutrition, 138(12), 2454-2461. doi: 10.3945/jn. 108.092163

Barrio-Lopez, M., Martinez-Gonzalez, M., Fernandez-Montero, A., Beunza, J., Zazpe, I. and Bes-Rastrollo, M. (2013). Prospective study of changes in sugar-sweetened beverage consumption and the incidence of the metabolic syndrome and its components: the SUN cohort. British Journal of Nutrition, 110(09), pp.1722-1731.

Bawa, S. (2005) The role of the consumption of beverages in the obesity epidemic, The Journal of The Royal Society for the Promotion of Health, 125, $124-8$.

Bazzano, L., Li, T., Joshipura, K. and Hu, F. (2008). Intake of Fruit, Vegetables, and Fruit Juices and Risk of Diabetes in Women. Diabetes Care, 31(7), pp.1311-1317.

Beck, J., Kassirer, J. and Pauker, S., (1982). A convenient approximation of life expectancy (the "DEALE”). The American Journal of Medicine, 73(6), pp.883-888.

Best, J., Drury, P., Davis, T., Taskinen, M., Kesaniemi, Y., Scott, R., Pardy, C., Voysey, M. and Keech, A. (2012). Glycemic Control Over 5 Years in 4,900 People With Type 2 Diabetes: Real-world diabetes therapy in a clinical trial cohort. Diabetes Care, 35(5), pp.1165-1170.

Bernstein, A., de Koning, L., Flint, A., Rexrode, K. and Willett, W. (2012). Soda consumption and the risk of stroke in men and women. American Journal of Clinical Nutrition, 95(5), pp.1190-1199.

Bleich, S., Segal, J., Wu, Y., Wilson, R. and Wang, Y. (2013). Systematic Review of Community-Based Childhood Obesity Prevention Studies. PEDIATRICS, 132(1), pp.e201-e210.

Bollard, Tessa, Ninya Maubach, Natalie Walker, and Cliona Ni Mhurchu. 2016. 'Effects of Plain Packaging, Warning Labels, and Taxes on Young People's Predicted 
Sugar-Sweetened Beverage Preferences: An Experimental Study'. International Journal of Behavioral Nutrition and Physical Activity 13 (September): 95. doi:10.1186/s12966-016-0421-7

Bonnet, C. and Réquillart, V. (2013). Tax incidence with strategic firms in the soft drink market. Journal of Public Economics, 106, pp.77-88.

Bosi, E., Camisasca, R., Collober, C., Rochotte, E. and Garber, A. (2007). Effects of Vildagliptin on Glucose Control Over 24 Weeks in Patients With Type 2 Diabetes Inadequately Controlled With Metformin. Diabetes Care, 30(4), pp.890-895.

Boyle, K., Welsh, M. and Bishop, R., (1993). The Role of Question Order and Respondent Experience in Contingent-Valuation Studies. Journal of Environmental Economics and Management, 25(1), pp.S80-S99.

Briefel, R., Wilson, A., \& Gleason, P. (2009). Consumption of Low-Nutrient, Energy-Dense Foods and Beverages at School, Home, and Other Locations among School Lunch Participants and Nonparticipants. Journal Of The American Dietetic Association, 109(2), S79-S90. doi: 10.1016/j.jada.2008.10.064

Briggs, A., Mytton, O., Kehlbacher, A., Tiffin, R., Rayner, M. and Scarborough, P. (2013). Overall and income specific effect on prevalence of overweight and obesity of $20 \%$ sugar sweetened drink tax in UK: econometric and comparative risk assessment modelling study. BMJ, 347(oct31 4), pp.f6189-f6189.

Briggs, A., Mytton, O., Madden, D., O’Shea, D., Rayner, M. and Scarborough, P. (2013). The potential impact on obesity of a $10 \%$ tax on sugar-sweetened beverages in Ireland, an effect assessment modelling study. BMC Public Health, 13(1).

Brooks Neil and Thaddeus Hwong (2006). The Social Benefits and Economics Costs of Taxation. Canadian Centre for Policy Alternative. pp 5-7. ISBN 0-88627-514-8.

Brownell, K., Farley, T., Willett, W., Popkin, B., Chaloupka, F., Thompson, J. and Ludwig, D. (2009). The Public Health and Economic Benefits of Taxing 
Sugar-Sweetened Beverages. New England Journal of Medicine, 361(16), pp.1599-1605.

Burgess, E., Hassmén, P., Welvaert, M. and Pumpa, K. (2017). Behavioural treatment strategies improve adherence to lifestyle intervention programmes in adults with obesity: a systematic review and meta-analysis. Clinical Obesity, 7(2), pp.105-114.

Cabrera Escobar, M., Veerman, J., Tollman, S., Bertram, M. and Hofman, K. (2013). Evidence that a tax on sugar sweetened beverages reduces the obesity rate: a meta-analysis. BMC Public Health, 13(1).

Calcott, P. and Petkov, V., (2015). Cigarette Taxes with Endogenous Addictiveness. The B.E. Journal of Theoretical Economics, 15(1).

Cancer care coordinators in stage III colon cancer: a cost-utility analysis. (2015). BioMed Central Ltd.

Caraher, M., \& Cowburn, G. (2005). Taxing food: implications for public health nutrition. Public Health Nutrition, 8(8), 1242-1249. doi: 10.1079/phn2005755

Cawley, J. and Frisvold, D. (2016). The Pass-Through of Taxes on Sugar-Sweetened Beverages to Retail Prices: The Case of Berkeley, California. Journal of Policy Analysis and Management, 36(2), pp.303-326.

Cawley, J., \& Meyerhoefer, C. (2012). The medical care costs of obesity: An instrumental variables approach. Journal Of Health Economics, 31(1), 219-230. doi: 10.1016/j.jhealeco.2011.10.003

Cawley, J., Thow, A., Wen, K., \& Frisvold, D. (2019). The Economics of Taxes on Sugar-Sweetened Beverages: A Review of the Effects on Prices, Sales, Cross-Border Shopping, and Consumption. Annual Review Of Nutrition, 39(1), 317-338. doi: 10.1146/annurev-nutr-082018-124603

Chan, W., Wright, C., Tobias, M., Mann, S. and Jackson, R. (2008). Explaining trends 
in coronary heart disease hospitalisations in New Zealand: trend for admissions and incidence can be in opposite directions. Heart, 94(12), pp.1589-1593.

Chun, S., Choi, Y., Chang, Y., Cho, J., Zhang, Y., Rampal, S., Zhao, D., Ahn, J., Suh, B., Pastor-Barriuso, R., Lima, J., Chung, E., Shin, H., Guallar, E. and Ryu, S. (2016). Sugar-sweetened carbonated beverage consumption and coronary artery calcification in asymptomatic men and women. American Heart Journal, 177, pp.17-24.

Claesson, L., Gosman-Hedstro “ m, G., Johannesson, M., Fagerberg, B. and Blomstrand, C. (2000). Resource Utilization and Costs of Stroke Unit Care Integrated in a Care Continuum: A 1-Year Controlled, Prospective, Randomized Study in Elderly Patients. Stroke, 31(11), pp.2569-2577.

Clarke, P., Gray, A., Briggs, A., Farmer, A., Fenn, P., \& Stevens, R. et al. (2004). A model to estimate the lifetime health outcomes of patients with Type 2 diabetes: the United Kingdom Prospective Diabetes Study (UKPDS) Outcomes Model (UKPDS no. 68). Diabetologia, 47(10), 1747-1759. doi: 10.1007/s00125-004-1527-z

Cobiac, L., Tam, K., Veerman, L. and Blakely, T. (2017). Taxes and Subsidies for Improving Diet and Population Health in Australia: A Cost-Effectiveness Modelling Study. PLOS Medicine, 14(2), p.e1002232.

Colantuoni, F. (2014). Empirical Studies On The Effectiveness Of Soda Taxes To Curb Obesity. Open Access.

Colantuoni, F. and Rojas, C. (2015). The impact of soda sales taxes on consumption: evidence from scanner data. Contemporary Economic Policy, 33(4), pp.714-734.

Colchero, M., Salgado, J., Unar-Munguía, M., Molina, M., Ng, S. and Rivera-Dommarco, J. (2015). Changes in Prices After an Excise Tax to Sweetened Sugar Beverages Was Implemented in Mexico: Evidence from Urban Areas. PLOS ONE, 10(12), p.e0144408. 
Colchero, M., Salgado, J., Unar-Munguía, M., Hernández-Ávila, M. and Rivera-Dommarco, J. (2015). Price elasticity of the demand for sugar sweetened beverages and soft drinks in Mexico. Economics \& Human Biology, 19, pp.129-137.

Colchero, M., Popkin, B., Rivera, J. and Ng, S. (2016). Beverage purchases from stores in Mexico under the excise tax on sugar sweetened beverages: observational study. BMJ, p.h6704.

Corina Grey, Rod Jackson, Susan Wells, Billy Wu, Katrina Poppe, Matire Harwood, Gerhard Sundborn, Andrew J Kerr. (2018). Trends in ischaemic heart disease: patterns of hospitalisation and mortality rates differ by ethnicity (ANZACS-QI 21). NZMJ, Vol 131, No 1478, ISSN 1175-8716.

Cornelsen, L., Mytton, O., Adams, J., Gasparrini, A., Iskander, D., Knai, C., Petticrew, M., Scott, C., Smith, R., Thompson, C., White, M. and Cummins, S. (2017). Change in non-alcoholic beverage sales following a 10-pence levy on sugar-sweetened beverages within a national chain of restaurants in the UK: interrupted time series analysis of a natural experiment. Journal of Epidemiology and Community Health, pp.jech-2017-209947.

Cornelsen, L., Adams, J., Gasparrini, A., Iskander, D., Knai, C., Mytton, O., Petticrew, M., Scott, C., Smith, R., Thompson, C., White, M. and Cummins, S. (2016). Impact of a levy on sales of sugar-sweetened beverages within a national chain of restaurants: interrupted time-series analysis. The Lancet, 388, p.S15.

Creedy, J. (2016). Sugar Taxes and Changes in Total Calorie Consumption: A Simple Framework. New Zealand Treasury Working Paper 16/06 December 2016.

Cox S. (2016). Annual Update of Key Results 2015/16: New Zealand Health Survey. Wellington: Ministry of Health; 2016.

de Koning, L., Malik, V., Rimm, E., Willett, W. and Hu, F. (2011). Sugar-sweetened and artificially sweetened beverage consumption and risk of type 2 diabetes in men. American Journal of Clinical Nutrition, 93(6), pp.1321-1327. 
de Koning, L., Malik, V., Kellogg, M., Rimm, E., Willett, W. and Hu, F. (2012). Sweetened Beverage Consumption, Incident Coronary Heart Disease, and Biomarkers of Risk in Men. Circulation, 125(14), pp.1735-1741.

Dharmasena S., Davis G., Capps O. (2012) Partial versus General Equilibrium Calorie and Revenue Effects Associated with a Sugar-Sweetened Beverage Tax. Journal of Agricultural and Resource Economics 39(2):157-173.

Diabetes Surveillance: population-based estimates and projections for New Zealand, 2001-2011 (2012). Public Health Intelligence Occasional Bulletin, No.46.

Duffey KJ, Gordon-Larsen P, Shikaney JM, Guilkey D, Jacobs DR, Popkin BM. (2010). Food price and diet and health outcomes. Archives of Internal Medicine 170(5): 420-426.

Eshak, E., Iso, H., Kokubo, Y., Saito, I., Yamagishi, K., Inoue, M. and Tsugane, S. (2012). Soft drink intake in relation to incident ischemic heart disease, stroke, and stroke subtypes in Japanese men and women: the Japan Public Health Centre-based study cohort I. American Journal of Clinical Nutrition, 96(6), pp.1390-1397.

Etilé, F. and Sharma, A. (2015). Do High Consumers of Sugar-Sweetened Beverages Respond Differently to Price Changes? A Finite Mixture IV-Tobit Approach. Health Economics, 24(9), pp.1147-1163.

Falbe, J., Rojas, N., Grummon, A. and Madsen, K. (2015). Higher Retail Prices of Sugar-Sweetened Beverages 3 Months After Implementation of an Excise Tax in Berkeley, California. American Journal of Public Health, 105(11), pp.2194-2201.

Finkelstein EA, Ruhm CJ, Kosa KM. (2005). Economic causes and consequences of obesity. Annu Rev Public Health 2005;26:239-57.

Finkelstein EA, Trogdon JF, Cohen JW, Dietz W. (2009). Annual medical spending attributable to obesity: Payer- and service-specific estimates. Health Affairs 
Finkelstein, E., Zhen, C., Bilger, M., Nonnemaker, J., Farooqui, A. and Todd, J. (2013). Implications of a sugar-sweetened beverage (SSB) tax when substitutions to non-beverage items are considered. Journal of Health Economics, 32(1), pp.219-239.

Fletcher, J.M., Frisvold, D.E. and Tefft, N. (2010) The effects of soft drink taxes on child and adolescent consumption and weight outcomes. Journal of Public Economics, 94, pp. 967-074.

Fletcher, J., Frisvold, D. and Tefft, N. (2014). Non-Linear Effects of Soda Taxes on Consumption and Weight Outcomes. Health Economics, 24(5), pp.566-582.

Foley, J., Bunck, M., Möller-Goede, D., Poelma, M., Nijpels, G., Eekhoff, E., Schweizer, A., Heine, R. and Diamant, M. (2011). Beta cell function following 1 year vildagliptin or placebo treatment and after 12 week washout in drug-naive patients with type 2 diabetes and mild hyperglycaemia: a randomised controlled trial. Diabetologia, 54(8), pp.1985-1991.

Friedman R., Brownell K. (2012). Rudd Report: Sugar-Sweetened Beverage Taxes: An Updated Policy Brief. Available from www.yaleruddcenter.org

Fung, T., Malik, V., Rexrode, K., Manson, J., Willett, W. and Hu, F. (2009). Sweetened beverage consumption and risk of coronary heart disease in women. American Journal of Clinical Nutrition, 89(4), pp.1037-1042.

Goodstein, E. (2011) Measuring the benefits of environment protection. Economics and environment, 6edn, Wiley, 142-167

Gray, A. and Clarke, P. (2008). The economic analyses of the UK prospective diabetes study. Diabetic Medicine, 2008, 25:47-51.

Green, D., O, N., Ducorroy, G., Skelly, A., Keegan, D., Kenny, D., Naughton, A. and Keegan, D. (2014). The Cost of Blindness in the Republic of Ireland 
2010-2020. Value in Health, 17(7), p.A607.

Griffith, R., O’Connell, M., \& Smith, K. (2017). Corrective Taxation and Internalities from Food Consumption. CESifo Economic Studies, 64(1), 1-14.

Gruber, J. and B. Koszegi (2002), “A Theory of Government Regulation of Addictive Bads:

Optimal Tax Levels and Tax Incidence for Cigarette Excise Taxation”, Working Paper w8777,

National Bureau of Economic Research.

Grummon, A., Lockwood, B., Taubinsky, D., \& Allcott, H. (2019). Designing better sugary drink taxes. Science, 365(6457), 989-990. doi: 10.1126/science.aav5199

Guala, F. (1999). The problem of external validity (or "parallelism") in experimental economics. Social Science Information, 38(4), pp.555-573.

Gustavsen, G., \& Rickertsen, K. (2011). The effects of taxes on purchases of sugar-sweetened carbonated soft drinks: a quantile regression approach. Applied Economics, 43(6), 707-716. doi: 10.1080/00036840802599776

Guerrero-López, C., Unar-Munguía, M. and Colchero, M. (2017). Price elasticity of the demand for soft drinks, other sugar-sweetened beverages and energy dense food in Chile. BMC Public Health, 17(1).

Herald on Sunday. (2016). Niki Bezzant: Sugary Drinks Tax - It's Time. [Internet]. Auckland, New Zealand: New Zealand Herald. 2016 [Updated 2016 Mar 27: Accessed 2017 June 28]. Available from:

http://www. nzherald.co.nz/lifestyle/news/article.cfm?c_id=6\&-objectid=11612259.

$\mathrm{Hu}$, F. (2013). Resolved: there is sufficient scientific evidence that decreasing sugar-sweetened beverage consumption will reduce the prevalence of obesity and obesity-related diseases. Obesity Reviews, 14(8), pp.606-619. 
Huffman, M. (2012). Association or Causation of Sugar-Sweetened Beverages and Coronary Heart Disease: Recalling Sir Austin Bradford Hill. Circulation, 125(14), pp.1718-1720.

Jacobson M, Brownell KD. (2000). Small taxes on soft drinks and snack foods to promote health. Am J Pub Health 2000;90:854-857.

Jacobsen, M. R., Knittel, C. R., Sallee, J. M., \& Van Benthem, A. A. (2016). Sufficient statistics for imperfect externality-correcting policies (No. w22063). National Bureau of Economic Research.

Jennum et al. (2015). Cost of stroke: a controlled national study evaluating societal effects on patients and their partners. BMC Health Serv Res; 15:466. doi:10.1186/s12913-015-1100-0.

Jonathan R. Godfrey, Deborah M. Brunning. (2009). Reconciling true and incurred costs of blindness in New Zealand. Social Policy Journal of New Zealand, Issue 36.

Jou, J., Niederdeppe, J., Barry, C., \& Gollust, S. (2014). Strategic Messaging to Promote Taxation of Sugar-Sweetened Beverages: Lessons From Recent Political Campaigns. American Journal of Public Health,104(5), 847-853. doi: 10.2105/ajph.2013.301679.

Keller, A., Heitmann, B. and Olsen, N. (2014). Sugar-sweetened beverages, vascular risk factors and events: a systematic literature review. Public Health Nutrition, 18(07), pp.1145-1154.

Khan, K., Kunz, R., Kleijnen, J. and Antes, G.. (2011). Systematic reviews to support evidence-based medicine. n.p.:CRC Pres.

Kifer, A. (2014). The Incidence of a Soda Tax, in Pennies and Pounds. SSRN Electronic Journal.

Kim, S., Park, S. and Lin, M.. (2016). Permanent tooth loss and sugar-sweetened 
beverage intake in U.S. young adults. Journal of Public Health Dentistry, 77(2), pp.148-154.

Klose, T. (1999). The contingent valuation method in health care. Health Policy, 47(2), pp.97-123.

Kyle, W. (2012). Pulmonary Hypertension Associated with Congenital Heart Disease: A Practical Review for the Pediatric Cardiologist. Congenital Heart Disease, 7(6), pp.575-583.

Lal, A., Mantilla-Herrera, A., Veerman, L., Backholer, K., Sacks, G., Moodie, M., Siahpush, M., Carter, R. and Peeters, A. (2017). Modelled health benefits of a sugar-sweetened beverage tax across different socioeconomic groups in Australia: A cost-effectiveness and equity analysis. PLOS Medicine, 14(6), p.e1002326.

Lal, A., Moodie, M., Ashton, T., Siahpush, M. and Swinburn, B. (2012). Health care and lost productivity costs of overweight and obesity in New Zealand. Australian and New Zealand Journal of Public Health, 36(6), pp.550-556.

Langer, L. and Stewart, R., 2014. What have we learned from the application of systematic review methodology in international development?-a thematic overview. Journal of Development Effectiveness, 6(3), pp.236-248.

Larsson, S., Akesson, A. and Wolk, A. (2014). Sweetened Beverage Consumption Is Associated with Increased Risk of Stroke in Women and Men. Journal of Nutrition, 144(6), pp.856-860.

Liljas, B. and Blumenschein, K. (2000). On hypothetical bias and calibration in costbenefit studies. Health Policy, 52(1), pp.53-70.

Long, M., Gortmaker, S., Ward, Z., Resch, S., Moodie, M., Sacks, G., Swinburn, B., Carter, R. and Claire Wang, Y. (2015). Cost Effectiveness of a Sugar-Sweetened Beverage Excise Tax in the U.S. American Journal of Preventive Medicine, 49(1), pp.112-123. 
Loomis J., Bell P., Cooney H., and Asmus C (2009). "A Comparison of Actual and Hypothetical Willingness to Pay of Parents and Non-Parents for Protecting Infant Health: The Case of Nitrates in Drinking Water.” Journal of Agricultural and Applied Economics, 41, 3:697-712 2009.

Ludwig DS, Peterson KE, Gortmaker SL. (2001). Relation between consumption of sugar-sweetened drinks and childhood obesity: A prospective, observational analysis. Lancet 2001;357:505-508.

Malik, V. (2017). Sugar sweetened beverages and cardiometabolic health. Current Opinion in Cardiology, p.1.

Malik, V., Popkin, B., Bray, G., Despres, J., Willett, W. and Hu, F. (2010). Sugar-Sweetened Beverages and Risk of Metabolic Syndrome and Type 2 Diabetes: A meta-analysis. Diabetes Care, 33(11), pp.2477-2483.

Manyema, M., Veerman, L., Chola, L., Tugendhaft, A., Sartorius, B., Labadarios, D. and Hofman, K. (2014). The Potential Impact of a 20\% Tax on Sugar-Sweetened Beverages on Obesity in South African Adults: A Mathematical Model. PLoS ONE, 9(8), p.e105287.

Manyema, M., Veerman, L., Tugendhaft, A., Labadarios, D. and Hofman, K. (2016). Modelling the potential impact of a sugar-sweetened beverage tax on stroke mortality, costs and health-adjusted life years in South Africa. BMC Public Health, 16(1).

Mekonnen, T., Odden, M., Coxson, P., Guzman, D., Lightwood, J., Wang, Y. and Bibbins-Domingo, K. (2013). Health Benefits of Reducing Sugar-Sweetened Beverage Intake in High Risk Populations of California: Results from the Cardiovascular Disease (CVD) Policy Model. PLoS ONE, 8(12), p.e81723.

Meyer, S., Yu, X., Abler, D., (2011). Comparison of several demand systems. In: Agricultural and Applied Economics Association 2011 Annual Meeting 103736, From: http://EconPapers.repec.org/RePEc:ags:aaea11:103736.http://refhub.elsevier.com/S15 
70-677X (15)00061-1/sbref0140.

Marron, D. (2015). Should We Tax Internalities Like Externalities?. SSRN Electronic Journal. doi: 10.2139/ssrn.2688672

Ministry of Health (2013). New Zealand Health Survey: Annual update of key findings 2012/13. Wellington: Ministry of Health, 2013.

Mitchell, Robert C. and Richard T. Carson. (1989). Using Surveys to Value Public Goods: The Contingent Valuation Method, Washington, DC: Johns Hopkins Press for Resources for the Future.

Montonen, J., Järvinen, R., Knekt, P., Heliövaara, M., \& Reunanen, A. (2007). Consumption of Sweetened Beverages and Intakes of Fructose and Glucose Predict Type 2 Diabetes Occurrence. The Journal Of Nutrition, 137(6), 1447-1454. doi: $10.1093 / \mathrm{jn} / 137.6 .1447$

Narain, A., Kwok, C. and Mamas, M. (2016). Soft drinks and sweetened beverages and the risk of cardiovascular disease and mortality: a systematic review and meta-analysis. International Journal of Clinical Practice, 70(10), pp.791-805.

National Health Committee Strategic. (2013). Strategic Overview: Cardiovascular Disease in New Zealand. National Health Committee Strategic to the Ministry of Health.

New Zealand Nephrology $12^{\text {th }}$ Annual Report: reporting on treatment practice and patient outcomes of dialysis and kidney transplantation in Aotearoa New Zealand in 2017. (2018). Australia \& New Zealand Dialysis \& Transplant Registry: National Renal Advisory Board.

New Zealand Guidelines Group. (2012). New Zealand Primary Care Handbook 2012.

Nielsen Company. (2016). New Zealand Media Trends Report 2016 [Internet]. New York: Neilsen Company; 2016. [Updated 2016 June 14; cited 2017 June 28]. 
http://www.nielsen.com/nz/en/insights/reports/2016/new-zealand-media-trends-2016. $\underline{\mathrm{html}}$

Ni Murchu, C., Eyles, H., Schilling, C., Yang, Q., Kaye-Blake, W., Genc,, M. and Blakely, T. (2013) Food prices and consumer demand: differences across income levels and ethnic groups. Plos One, 8, pp. 1-12. Available from: http://journals.plos.org/plosone/article?id=10.1371/journal.pone.0075934

NZIER. (2018). The Social and Economic Costs of Stroke in New Zealand. NZIER report to the Stroke Foundation.

O’Donoghue, Ted, and Matthew Rabin. 2006. “Optimal Sin Taxes.” Journal of Public Economics 90 (10-11): 1825-49.

Ogden CL, Kit BK, Carroll MD, Park S. (2011). Consumption of sugar drinks in the United States, 2005 - 2008. No. 71. Hyattsville, MD: National Center for Health Statistics. (DHHS publication no. (PHS) 2011-1209.)

Olsen, J., \& Smith, R. (2001). Theory versuspractice: a review of ?willingness-to-pay? in health and health care.Health Economics, 10(1), 39-52. doi: 10.1002/1099-1050(200101)10:1<39::aid-hec563>3.0.co;2-e.

Palmer JR, Boggs DA, Krishnan S, Hu FB, Singer M, Rosenberg L. (2008). Sugar-sweetened beverages and incidence of type 2 diabetes mellitus in African American women. Arch Intern Med. 2008;168:1487-1492.

Paserman, M. (2008). Job Search and Hyperbolic Discounting: Structural Estimation and Policy Evaluation*. The Economic Journal, 118(531), pp.1418-1452.

Pemberton, C., Harris-Charles, E. and Patterson-Andrews, H. (2010). Cultural bias in contingent valuation of copper mining in the Commonwealth of Dominica. Ecological Economics, 70(1), pp.19-23. 
Popkin, B. (2010). Patterns of beverage use across the lifecycle. Physiology \& Behavior, 100(1), 4-9. doi: 10.1016/j.physbeh.2009.12.022

Powell, L., \& Chaloupka, F. (2009). Food Prices and Obesity: Evidence and Policy Implications for Taxes and Subsidies. Milbank Quarterly, 87(1), 229-257. doi: 10.1111/j.1468-0009.2009.00554.x

Robinson, L., Hammitt, J. (2011). Behavioral Economics and the Conduct of Benefit-Cost Analysis: Towards Principles and Standards. Journal of Benefit-Cost Analysis, 2(2).

Sánchez-Romero, L., Penko, J., Coxson, P., Fernández, A., Mason, A., Moran, A., Ávila-Burgos, L., Odden, M., Barquera, S. and Bibbins-Domingo, K. (2016). Projected Impact of Mexico's Sugar-Sweetened Beverage Tax Policy on Diabetes and Cardiovascular Disease: A Modeling Study. PLOS Medicine, 13(11), p.e1002158.

Schulze, M., Manson, J., \& Ludwig, D. (2004). Sugar-sweetened beverages, weight gain, and incidence of type 2 diabetes in young and middle-aged women. $A C C$ Current Journal Review, 13(11), 34-35. doi: 10.1016/j.accreview.2004.10.018

Schwendicke, F. and Stolpe, M. (2017). Taxing sugar-sweetened beverages: impact on overweight and obesity in Germany. BMC Public Health, 17(1).

Scott M., Peter M., Hew N., Dilky R. (2014). Self-monitoring blood glucose test strip use with diabetes medicines in people with types 1 and 2 diabetes in New Zealand. NZMJ 28 November 2014, Vol 127 No 1406; ISSN 1175-8716, pp.48.

Sgarbieri, V., \& Pacheco, M. (2017). Healthy human aging: intrinsic and environmental factors. Brazilian Journal of Food Technology,20(0). doi: $10.1590 / 1981-6723.00717$

Sharma, A., Hauck, K., Hollingsworth, B. and Siciliani, L. (2014). THE EFFECTS OF TAXING SUGAR-SWEETENED BEVERAGES ACROSS DIFFERENT 
INCOME GROUPS. Health Economics, 23(9), pp.1159-1184.

Silver, L., Ng, S., Ryan-Ibarra, S., Taillie, L., Induni, M., Miles, D., Poti, J. and Popkin, B. (2017). Changes in prices, sales, consumer spending, and beverage consumption one year after a tax on sugar-sweetened beverages in Berkeley, California, US: A before-and-after study. PLOS Medicine, 14(4), p.e1002283.

Smith, T. G.; Chouinard, H. H. and Wandschneider, P. R. (2011). Waiting for the invisible hand: Novel products and the role of information in the modern market for food, Food Policy 36 : 239- 249.

Smith, R., \& Sach, T. (2010). Contingent valuation: what needs to be done? Health Economics, Policy And Law, 5(1), 91-111. doi: 10.1017/s1744133109990016

Smith TA, Lin B-H, Lee J-Y. (2010). Taxing caloric sweetened beverages: potential effects on beverage consumption, calorie intake and obesity. ERR-100, U.S. Department of Agriculture, Economic Research Service.

Stalhammar, N. (1996). An Empirical Note on Willingness to Pay and Starting-point Bias. Medical Decision Making, 16(3), pp.242-247.

Snyder C., Nicholson W. (2015). Microeconomic theory: basic principles and extensions.

Tandel, K., (2011). Sugar substitutes: Health controversy over perceived benefits. Journal of Pharmacology and Pharmacotherapeutics, 2(4), p.236.

Tiffany P., Charlotte J., Ishika J. (2017). Sweet rebellion: a campaign for a sugar-sweetened beverage tax in New Zealand. NZMJ 1 September 2017, Vol 130 No 1461 ISSN 1175-8716 C NZMA, www.nzma.org.nz/journal

Tiffin, R., Kehlbacher, A. and Salois, M. (2014). The Effects of A Soft Drink Tax in the UK. Health Economics, 24(5), pp.583-600. 
Tobias, M., Cheung, J., Carter, K., Anderson, C. and Feigin, V. (2007). Stroke surveillance: population-based estimates and projections for New Zealand. Australian and New Zealand Journal of Public Health, 31(6), pp.520-525.

Tummers, J., Schrijvers, A., \& Visser-Meily, A. (2012). Economic evidence on integrated care for stroke patients; a systematic review. International Journal of Integrated Care, 12(8). doi: 10.5334/ijic. 847

Turner R. C., Holman R. R., Mattews D. R. (1991). UK Prospective Diabetes Study (UKPDS): study design, progress, and performance. Diabetologia, 1991, 34(12): 877-890.

Ungureanu, I., Dranga, M., Didita, A., Prelipcean, C. and Mihai, C. (2016). Obesity, risk factor for severe acute pancreatitis. Pancreatology, 16(4), p.S164.

Vargas-Garcia, E., Evans, C., Prestwich, A., Sykes-Muskett, B., Hooson, J. and Cade, J. (2017). Interventions to reduce consumption of sugar-sweetened beverages or increase water intake: evidence from a systematic review and meta-analysis. Obesity Reviews, 18(11), pp.1350-1363.

Veerman, J., Sacks, G., Antonopoulos, N. and Martin, J. (2016). The Impact of a Tax on Sugar-Sweetened Beverages on Health and Health Care Costs: A Modelling Study. PLOS ONE, 11(4), p.e0151460.

Wang, E. (2015). The impact of soda taxes on consumer welfare: implications of storability and taste heterogeneity. The RAND Journal of Economics, 46(2), pp.409-441.

Wang, Y., Coxson, P., Shen, Y., Goldman, L., \& Bibbins-Domingo, K. (2012). A Penny-Per-Ounce Tax On Sugar-Sweetened Beverages Would Cut Health And Cost Burdens Of Diabetes. Health Affairs, 31(1), 199-207. doi: 10.1377/hlthaff.2011.0410

Waterlander, W., Ni Mhurchu, C. and Steenhuis, I. (2014). Effects of a price increase on purchases of sugar sweetened beverages. Results from a randomized controlled 
trial. Appetite, 78, pp.32-39.

What drives sugar addiction. (2015). Nature, 518(7537), pp.8-8.

WHO 2020. Obesity And Overweight. [online] Available at: $<$ https://www.who.int/news-room/fact-sheets/detail/obesity-and-overweight $>$ [Accessed 2 October 2020].

Wong, C., Tang, E. and Herbison, P.. (2007). Survival Over 5 Years in the Initial Hospital Survivors with Acute Coronary Syndrome: A Comparison Between a Community Hospital and a Tertiary Hospital in New Zealand. Heart, Lung and Circulation, 16, p.S173.

Woolcock, M., Bamberger, M. and Rao, V. (2010). Using Mixed Methods in Monitoring and Evaluation: Experiences from International Development. SSRN Electronic Journal.

Yong, Pierre L, John Bertko, and Richard Kronick. (2011). Actuarial Value and Employer - Sponsored Insurance. ASPE Research Brief, US Department of Health and Human Services.

Zhen, C., Finkelstein, E., Nonnemaker, J., Karns, S. and Todd, J. (2014) By ounce or by calorie, differential effects of alternative sugar-sweetened beverage tax strategy. American Journal of Agricultural economics, 96(4): 1070-10

Zhen, C., Finkelstein, E., Nonnemaker, J., Karns, S. and Todd, J. (2013). Predicting the Effects of Sugar-Sweetened Beverage Taxes on Food and Beverage Demand in a Large Demand System. American Journal of Agricultural Economics, 96(1), pp.1-25. 